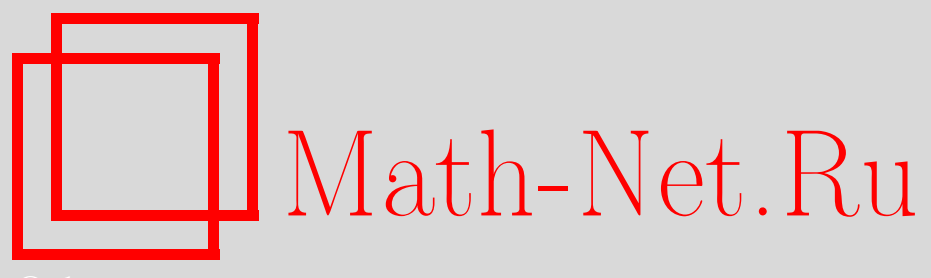

Е. М. Чирка, Голоморфные движения и униформизация голоморфных семейств римановых поверхностей, УМH, 2012, том 67, выпуск 6, 125-202

DOI: https://doi.org/10.4213/rm9499

Использование Общероссийского математического портала Math-Net.Ru подразумевает, что вы прочитали и согласны с пользовательским соглашением http://www . mathnet.ru/rus/agreement

Параметры загрузки:

IP : 18.234 .156 .22

26 апреля 2023 г., 14:18:27






\title{
Голоморфные движения и униформизация голоморфных семейств римановых поверхностей
}

\author{
Е. М. Чирка
}

Изучаются условия, достаточные для одновременной униформизации голоморфных семейств римановых поверхностей. Основной метод основан на локальной конструкции голоморфных и почти голоморфных движений с последующим применением техники пространств Тейхмюллера. Голоморфные движения применяются также к близкой задаче о продолжении комплексных структур.

Библиография: 62 названия.

Ключевые слова: римановы поверхности, пространства Тейхмюллера, униформизация, голоморфные движения, устранимые особенности.

\section{СОДЕРЖАНИЕ}

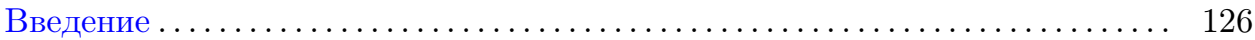

1. Голоморфные семейства римановых поверхностей ................ 127

1.1. Определения и комментарии к ним ................... 127

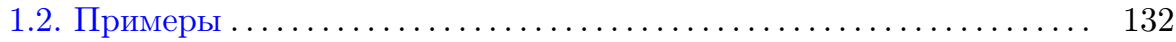

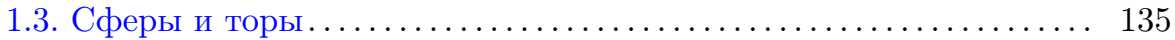

2. Голоморфные движения и униформизация .................. 141

2.1. Классические голоморфные движения................... 141

2.2. Горизонтально голоморфные тривиализации .............. 147

2.3. Униформизация локально тривиализуемых семейств ......... 153

3. Голоморфные движения и пространства Тейхмюллера . . . . . . . . 156

3.1. Универсальные семейства ........................... 156

3.2. Редукция базы ..................................... 160

3.3. Теорема Берса-Ройдена .......................... 164

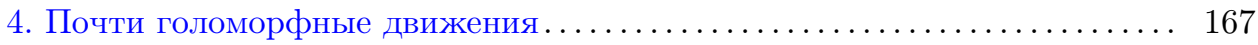

4.1. Квазилинейные уравнения Бельтрами . . . . . . . . . . . . . . . . . 167

4.2. Краевая задача Римана-Гильберта ...................... 173

5. Униформизация некоторых семейств ...................... 180

5.1. Компактные поверхности с проколами................... 180

5.2. Семейства с диск-плоскими границами .................. 185

5.3. Плоскости. Теорема Нишино . . . . . . . . . . . . . . . . . . . . . 187

Работа выполнена при поддержке РФФИ (гранты № 11-01-00495 и 11-01-12033-офи-м).

(C) Е. М. Чирка, 2012 
6. Об устранимых особенностях комплексных структур ............... 191

6.1. Семейства проколотых дисков ........................ 191

6.2. Устранение послойно дискретных особенностей ............. 193

6.3. Комментарии..................................... 197

Список литературы . . . . . . . . . . . . . . . . . . . . . . . . . 199

\section{Введение}

Основная тематика статьи - это одновременная униформизация голоморфных семейств римановых поверхностей. В отличие от индивидуальных римановых поверхностей, униформизация семейств далеко не всегда существует, и приводимые в статье положительные результаты в основном относятся к семействам, локально допускающим "горизонтально голоморфные" тривиализации, тесно связанные с униформизациями Берса над пространствами Тейхмюллера.

Основной инструмент построения униформизации при помощи расслоений Берса - это промежуточная конструкция так называемых голоморфных движений, т. е. семейств попарно непересекающихся голоморфных сечений проекции тотального пространства $M$ семейства $\left\{M_{\lambda}\right\}$ на базу параметров $B$. Как показано в работах Эрла-Фоулера [20], [21], для любого семейства гиперболических римановых поверхностей над односвязной базой локальная (по параметру) тривиализация голоморфными движениями является необходимым и достаточным условием голоморфности классифицирующего отображения базы в соответствующее пространство Тейхмюллера, а само семейство при наличии такой тривиализации является прообразом “универсальной кривой Тейхмюллера" относительно этого отображения.

Практически все результаты статьи относятся к топологически локально тривиальным семействам, но в разделе 1 обсуждается также общая ситуация и приводятся примеры, в том числе неуниформизуемых топологически локально тривиальных семейств римановых поверхностей.

Для семейств сфер (см. [37]) и торов техника униформизации сравнительно простая, условия униформизуемости зависят от голоморфных свойств базы и наличия глобальных голоморфных сечений (п. 1.3).

Разделы 2 и 3 составляют теоретическую основу униформизации при помощи голоморфных движений. В разделе 2 исследуются голоморфные движения в семействах римановых поверхностей и доказываются теоремы об униформизации семейств, которые локально тривиализуются голоморфными движениями (теорема 2.1). В разделе 3 подключается техника пространств Тейхмюллера, доказываются теорема Эрла-Фоулера [20] об универсальности и теорема Берса-Ройдена [12] о продолжении голоморфных движений.

В разделе 4 разрабатывается техника построения голоморфных движений, основанная на краевой задаче Римана-Гильберта для решений специальных квазилинейных уравнений Бельтрами; свойства этих решений исследуются в п. 4.1.

В п. 5.1 показано, как строятся локальные горизонтально голоморфные тривиализации для семейств компактных римановых поверхностей рода $>1$ (см. 
[40], [34]) и для компактных поверхностей с проколами (см. [19]; о сферах с проколами см. [12]). Условия униформизуемости семейств гиперболических римановых поверхностей принципиально отличаются от условий униформизуемости семейств параболических и рациональных поверхностей: для последних основными являются комплексные условия на базу (разрешимость проблем Кузена) и на существование глобальных сечений, а для семейств гиперболических поверхностей достаточно всего лишь односвязности базы, но зато нужны условия на тотальные пространства семейств типа локальной псевдовыпуклости.

Голоморфные семейства римановых поверхностей, конформно эквивалентных комплексной плоскости $\mathbb{C}$ или накрываемых $\mathbb{C}$ (параболические поверхности), представляют самостоятельный интерес. Основной здесь является теорема Нишино [49], утверждающая, что голоморфное семейство плоскостей, параметризуемых единичным кругом $\mathbb{D}$, биголоморфно эквивалентно $\mathbb{D} \times \mathbb{C}$, если пространство этого семейства является многообразием Штейна. В п. 5.3 приводится доказательство теоремы Нишино с помощью голоморфных движений, при более слабых условиях на тотальное пространство.

В п. 6.1 доказывается локальный аналог теоремы Нишино, который можно рассматривать как теорему о продолжении комплексных структур и как обобщение теоремы Гартогса об аналитичности особенностей. Этот результат обобщается в п. 6.2 на семейства кругов с конечными множествами проколов.

\section{1. Голоморфные семейства римановых поверхностей}

1.1. Определения и комментарии к ним. Голоморфное семейство римановых поверхностей - это тройка $\pi: M \rightarrow B$, где $M, B$ - комплексные многообразия, $\pi$ - голоморфное сюръективное отображение (проекция) и все слои $M_{\lambda}:=\pi^{-1}(\lambda)$ - римановы поверхности. При этом $M$ и $B$, которые всюду в дальнейшем предполагаются линейно связными, могут быть и бесконечномерными банаховыми многообразиями (например, в качестве $B$ у нас неоднократно фигурируют бесконечномерные пространства Тейхмюллера). Аналогичное определение можно привести и в более общей категории комплексных пространств (с особенностями), но нам оно не понадобится.

Многообразие $B$ называется базой голоморфного семейства (многообразием параметров), а $M-$ пространством семейства $\left\{M_{\lambda}\right\}$.

Голоморфные семейства римановых поверхностей образуют категорию; отображение семейства $\pi: M \rightarrow B$ в семейство $\pi^{\prime}: M^{\prime} \rightarrow B^{\prime}-$ это пара голоморфных отображений $f: M \rightarrow M^{\prime}$ и $g: B \rightarrow B^{\prime}$ таких, что $\pi^{\prime} \circ f=g \circ \pi$. Произвольные отображения $f: M \rightarrow M^{\prime}$ с этим свойством мы называем послойными. Семейства $\pi: M \rightarrow B, \pi^{\prime}: M^{\prime} \rightarrow B^{\prime}$ называем (биголоморфно) эквивалентными, если существуют соответствующие биголоморфные отображения $f, g$.

Если $g: B^{\prime} \rightarrow B$ - голоморфное отображение, то над $B^{\prime}$ естественно определяется семейство римановых поверхностей $M_{\lambda^{\prime}}^{\prime}:=M_{g\left(\lambda^{\prime}\right)}$, дизъюнктное объединение $M^{\prime}$ которых имеет однозначно определяемую структуру комплексного многообразия, относительно которой послойно тождественное отображение $f: M^{\prime} \rightarrow M, f \mid M_{\lambda^{\prime}}^{\prime} \stackrel{\text { id }}{\rightarrow} M_{g\left(\lambda^{\prime}\right)}$ голоморфно (голоморфные функции на $M^{\prime}$ 
в окрестности точки $p^{\prime}$ - это по определению прообразы голоморфных функций в окрестности $f\left(p^{\prime}\right)$ и функции вида $h \circ \pi^{\prime}$, где $h$ голоморфна в окрестности $\pi^{\prime}\left(p^{\prime}\right)$ и $\pi^{\prime} \mid M_{\lambda^{\prime}} \equiv \lambda^{\prime}-$ проекция). Семейство $\pi^{\prime}: M^{\prime} \rightarrow B^{\prime}$ называется прообразом $\pi: M \rightarrow B$ относительно отображения $g$.

Семейство $\pi: M \rightarrow B$ называется локально топологически (гладко, голоморфно) тривиальным, если для всякого $\lambda \in B$ существуют окрестность $U \ni \lambda$ и гомеоморфизм (диффеоморфизм, биголоморфизм) $\psi: U \times M_{\lambda} \rightarrow \pi^{-1}(U)$ такие, что $\pi \circ \psi$ есть проекция $U \times M_{\lambda}$ на первый прямой сомножитель. В такой ситуации $M$ является расслоенным пространством (топологическим, гладким, комплексным). В основном мы работаем далее с локально топологически тривиальными семействами.

Локально в $M$ всякое голоморфное семейство биголоморфно тривиально, точнее, справедливо следующее.

Лемма 1.1. Пусть $\pi: M \rightarrow B$ - голоморфное семейство некомпактных римановых поверхностей $M_{\lambda}$ над конечномерным многообразием В. Тогда для всякого $\lambda \in B$ и всякой области $D \Subset M_{\lambda}$ существуют окрестность $U \ni$ $\lambda$ и биголоморфное вложение (тривиализация) $h: U \times D \rightarrow M$ такие, что $h(0, p) \equiv p u \pi \circ h\left(\lambda^{\prime}, p\right)=\lambda^{\prime}$ для всех $\lambda^{\prime} \in U, p \in D$.

ДоказАтельство. Так как $M_{\lambda}$ некомпактна, то на ней есть голоморфная функция $f_{\lambda}$ такая, что $d f_{\lambda}$ не имеет нулей (глобальная локальная координата, см. [25]). Так как $M_{\lambda}$ - многообразие Штейна, то по теореме Сью [55] существует окрестность $V \supset M_{\lambda}$ в $M$, которая тоже является многообразием Штейна. А тогда на $V$ есть голоморфная функция $f$ такая, что $f \mid M_{\lambda}=f_{\lambda}$, и, значит, существуют окрестности $U \ni \lambda$ в $B$ и $W \supset D$ в $M$ такие, что каждая связная компонента уровня $f=$ const в $W$ проектируется на $U$ взаимно однозначно. Такую компоненту, содержащую точку $p \in W$, обозначим $s_{p}$ и положим $h^{-1}(p)=\left(\pi(p), s_{p} \cap M_{\lambda}\right)$. Таким образом определено очевидно голоморфное и взаимно однозначное (следовательно, биголоморфное) отображение $W$ на $U \times D$. Лемма доказана.

СЛЕДСТвИЕ 1.1. При условиях лемм, пусть X -замкнутое подмножество в $M$ такое, что сужение $\pi \mid X$ собственное (прообраз компакта - компакт). Тогда семейство голоморфных сечений $\pi$ над $B$ со значениями в $X$ компактно, т.е. из всякой последовательности таких сечений выделяется подпоследовательность, сходящаяся равномерно на компактных подмножествах в $B$.

ДокАЗАТЕльство. Пусть $\left\{s_{n}\right\}$ - последовательность таких сечений. Покроем $X$ счетным семейством областей $W_{j}$ из леммы 1.1 таких, что $U_{j}=\pi\left(W_{j}\right)-$ координатные шары и горизонтальная граница каждой $W_{j}$, т. е. множество $\partial W_{j} \cap \pi^{-1}\left(U_{j}\right)$, не пересекает $X$.

Так как $W_{j}$ - гиперболические многообразия по построению, то отображения $s_{n} \mid U_{j} \rightarrow W_{j}$ не увеличивают метрику Кобаяши и, таким образом, образуют равностепенно непрерывное семейство (топология, индуцируемая на гиперболическом многообразии метрикой Кобаяши, совпадает с обычной топологией). Поэтому в каждой $W_{j}$ из последовательности $\left\{s_{n} \mid U_{j}\right\}$ выделяется подпоследовательность, равномерно сходящаяся внутри $U_{j}$, а искомая подпоследовательность выделяется затем диагональным процессом. Следствие доказано. 
Униформизация комплексного $n$-мерного многообразия $M$ - это голоморфное накрытие его некоторой областью $\Omega$ в $\mathbb{C}^{n}$ или в каком-нибудь пополнении $\mathbb{C}^{n}$ типа $\mathbb{C P}_{n}$, т. е. представление $M$ в виде $\Omega / \Gamma$, где $\Gamma$ - дискретная группа голоморфных автоморфизмов $\Omega$ без неподвижных точек (см. [9]). Существование такого накрытия в какой-то мере упрощает анализ на $M$, сводя его к анализу соответствующих объектов на области $\Omega$ (где есть глобальные голоморфные или однородные координаты), с контролем инвариантности относительно группы $Г$.

Униформизуемость многомерных $(n>1)$ комплексных многообразий - событие довольно исключительное и эта проблема обычно формулируется как проблема открытой вложимости соответствующих универсальных накрытий в $\mathbb{C}^{n}$, $\mathbb{C P}_{n}$ и т. п. В одномерном случае ситуация кардинально проще: по теореме Пуанкаре-Кёбе всякая риманова поверхность униформизуется, ее универсальная накрывающая конформно (= биголоморфно) эквивалентна сфере Римана $\widehat{\mathbb{C}}=\mathbb{C P}_{1}$, комплексной плоскости $\mathbb{C}$ или единичному кругу $\mathbb{D} \subset \mathbb{C}$. Поэтому естественно воспользоваться такой замечательной особенностью одномерных многообразий для униформизации многомерных многообразий, используя их возможные представления в виде пространств, расслоенных голоморфными семействами римановых поверхностей, как выше.

В связи с этим (и с другими задачами, где фигурируют семейства римановых поверхностей) полезно решить предварительную задачу одновременной униформизации такого семейства $\pi: M \rightarrow B$, т. е. найти область $\Omega$ в $B \times \widehat{\mathbb{C}}$, послойно накрывающую $M$, точнее область $\Omega$ вместе с сюръективным голоморфным отображением $f: \Omega \rightarrow M$ таким, что $\pi \circ f-$ прямая проекция $\Omega$ на $B$ и $\Omega_{\lambda} \rightarrow M_{\lambda}-$ накрытие для каждого $\lambda \in B$ (т. е. все слои $\Omega_{\lambda}$ связные и у каждой точки $p \in M_{\lambda}$ есть окрестность $U_{\lambda} \ni p$ в $M_{\lambda}$ такая, что всякая связная компонента множества $f^{-1}\left(U_{\lambda}\right) \subset \Omega_{\lambda}$ проектируется на $U_{\lambda}$ взаимно однозначно). Всюду в дальнейшем под униформизацией семейств римановых поверхностей подразумевается именно такая одновременная униформизация.

Отображение $f$ не обязательно является накрытием (см. пример 1.1 в п. 2.1), но если семейство $\pi: M \rightarrow B$ топологически локально тривиальное, то послойное накрытие $\psi$ является также обычным накрытием $\Omega \rightarrow M$.

Отметим, что если семейство $\left\{M_{\lambda}\right\}$ не содержит сфер и допускает послойно универсальное накрытие (т.е. послойное накрытие с односвязными слоями), то последнее является локально гладко тривиальным семейством (см. [46]) и это позволяет подключить к проблеме униформизации технику почти комплексного анализа в $\mathbb{D} \times \mathbb{C}$ (см., скажем, пример 1.2).

Если $\pi: M \rightarrow B$ допускает непрерывное (не обязательно голоморфное) глобальное сечение $s$, то среди всех возможных послойных голоморфных накрытий (не обязательно областями в $B \times \widehat{\mathbb{C}}$ ) есть универсальный объект - накрытие, в которое поднимается сечение $s$ и которое послойно накрывает любое другое послойное накрытие с этим свойством. Это так называемое многообразие универсальных накрывающих $\widetilde{M}^{s}$ семейства $\pi: M \rightarrow B$. Его точками являются пары $(p,[\gamma])$, где $p$ - точка в $M$ и $[\gamma]$ - гомотопический класс путей на $M_{\pi(p)}$ с началом $s(\pi(p))$ и концом $p$. По лемме 1.1 , существуют окрестность $W$ пути $\gamma \subset M_{\lambda}, \lambda=\pi(p)$, в $M$ и голоморфный ретракт $r: W \rightarrow W \cap M_{\lambda}$. Пусть 
$V_{0}, V_{1}$ - односвязные окрестности в $M_{\lambda}$ точек $s(\lambda)$ и $p$ соответственно. Окрестность точки $(p,[\gamma])$ в $\widetilde{M}^{s}$ по определению есть множество точек $\left(q,\left[\gamma^{\prime}\right]\right)$ таких, что $q \in r^{-1}\left(V_{1}\right)$ и путь $r \circ \gamma^{\prime}$ гомотопен $\gamma$ на $M_{\lambda}$ в классе путей с началом в $V_{0}$ и концом в $V_{1}$. Эти окрестности образуют базис топологии на $\widetilde{M}^{s}$, относительно которой отображение (проекция) $\widetilde{M}^{s} \ni(p,[\gamma]) \mapsto p \in M$ непрерывно, причем у каждой точки из $\widetilde{M}^{s}$ есть окрестность, которая проектируется на свой образ в $M$ взаимно однозначно. Поэтому на $\widetilde{M}^{s}$ однозначно определена комплексная структура, которая является поднятием таковой из $M$ и однозначно определяется условием, что проекция $\tilde{\pi}: \widetilde{M}^{s} \rightarrow M$ голоморфна.

По построению, проекция $\tilde{\pi} \circ \pi$ допускает глобальное голоморфное сечение $\lambda \mapsto(s(\lambda),[s(\lambda)])$, где $[s(\lambda)]$ - класс замкнутых путей в $M_{\lambda}$ с началом $s(\lambda)$, гомотопных постоянному пути, причем любое сечение проекции $\pi: M \rightarrow B$, послойно гомотопное $s$, поднимается до сечения проекции $\tilde{\pi} \circ \pi: \widetilde{M}^{s} \rightarrow B$. Слои проекции $\tilde{\pi} \circ \pi$ являются универсальными накрывающими соответствующих $M_{\lambda}$ (отсюда название), и универсальность $\widetilde{M^{s}}$ в указанном классе послойных накрытий доказывается элементарно (нам она не понадобится).

Отметим, что многообразие $\widetilde{M}^{s}$ существенно зависит от гомотопического класса базового глобального сечения $s$ в $M$ (гомотопия послойная) и послойная униформизуемость или неуниформизуемость $\widetilde{M^{s}}$ может зависеть от того, какое глобальное сечение $s$ выбрано в качестве базового.

Важную роль в изучении голоморфных семейств римановых поверхностей играют различные понятия псевдовыпуклости (см., например, [61], [62], [44]). Самым слабым и относительно просто проверяемым условием такого типа является условие диск-выпуклости. Комплексное многообразие $M$ называется диск-выпуклым, если для любого компакта $K \subset M$ найдется компакт $\widehat{K} \subset M$ такой, что если $f: \mathbb{D} \rightarrow M$ - голоморфный диск с $f(\partial \mathbb{D}) \subset K$ (т. е. $f(\mathbb{D}) \cup K-$ компакт), то $f(\mathbb{D}) \subset \widehat{K}$. Для наших целей в основном достаточно еще более слабого условия, с весьма специальными голоморфными дисками.

Голоморфное семейство $\pi: M \rightarrow B$ назовем трансверсально диск-выпуклым, если для каждой точки $\lambda^{\prime} \in B$ существует окрестность $U^{\prime} \ni \lambda^{\prime}$ со следующим свойством: для всякого вложенного голоморфного диска $h: \overline{\mathbb{D}} \rightarrow U^{\prime}$ с $h(0)=\lambda^{\prime}$ и всякого компакта $K \in \pi^{-1}(h(\partial \mathbb{D}))$ найдется компакт $\widehat{K} \subset M$ такой, что если $f: \overline{\mathbb{D}} \rightarrow M-$ замкнутый голоморфный диск, $\pi \circ f=h$ ("горизонтальный" диск) и $f(\partial \mathbb{D}) \subset K$, то $f(\mathbb{D}) \subset \widehat{K}$. (Голоморфность $f$ на $\overline{\mathbb{D}}$ означает, что $f$ продолжается до голоморфного отображения $f: U \rightarrow M$ некоторого большего круга $U \supset \mathbb{D}$; замкнутый голоморфный диск - это отображение, непрерывное в $\overline{\mathbb{D}}$ и голоморфное в $\mathbb{D}$.)

Если для всякой последовательности замкнутых голоморфных дисков $f_{n}$ : $\overline{\mathbb{D}} \rightarrow M$, вложенных в слои $M_{\lambda}$ ("вертикальные" диски), из равномерной сходимости $f_{n} \mid \partial \mathbb{D}$ следует равномерная сходимость $f_{n}$ в $\mathbb{D}$, то семейство $\pi: M \rightarrow B$ будем называть вертикально диск-выпуклым.

Согласно следствию 1.1, условие трансверсальной диск-выпуклости эквивалентно следующему: если $U$ - окрестность из определения, $h: \overline{\mathbb{D}} \rightarrow U-$ вложенный голоморфный диск, $f_{n}: \overline{\mathbb{D}} \rightarrow M, n=0,1, \ldots,-$ замкнутые голоморфные диски с $\pi \circ f_{n}=h$ и $K-$ компакт в $M$ такой, что $f_{n}(\partial \mathbb{D}) \subset K$ для любого $n$, то 
можно выделить подпоследовательность $\left\{f_{n_{k}}\right\}$, равномерно внутри $\mathbb{D}$ сходящуюся к некоторому диску $f: \mathbb{D} \rightarrow \pi^{-1}(U)$, причем замыкание $f(\mathbb{D})$ в $M$ - тоже компакт.

Следующее граничное свойство голоморфных дисков весьма полезно в применениях этого свойства.

Лемма 1.2. Пусть $\pi: M \rightarrow U-$ голоморфное семейство римановых поверхностей над областъю $U \supset \overline{\mathbb{D}}$ в $\mathbb{C}_{\zeta}$. Пусть $f_{n}: \mathbb{D} \rightarrow M-$ голоморфные сечения проекиии $\pi$, равномерно внутри $\mathbb{D}$ сходящиеся $\kappa$ сечению $f: \mathbb{D} \rightarrow M$. Предположим, что существует компакт $K \subset \pi^{-1}(\partial \mathbb{D})$ такой, что все множества $K \cup f_{n}(\mathbb{D})$ компактны. Тогда для каждого $\zeta \in \partial \mathbb{D}$ множество предельных точек диска $f(\mathbb{D})$ на $M_{\zeta} \backslash K_{\zeta}$ либо пусто, либо является объединением некоторых связных компонент множества $M_{\zeta} \backslash K_{\zeta}$.

ДоказАтельство. Достаточно доказать утверждение для $\zeta=1$. Пусть $a \in$ $M_{1} \backslash K_{1}$ - предельная точка для $f(\mathbb{D})$ и $z$ - голоморфная функция в окрестности $a$ на $M$ такая, что $z(a)=0, d \zeta \wedge d z \neq 0$, т. е. $\zeta, z$ - голоморфные координаты в окрестности $V^{0} \ni a$ на $M$, не пересекающей $K$. Мы хотим показать, что $\overline{f(\mathbb{D})}$ содержит окрестность $a$ на $M_{1}$.

Предположим противное. Тогда существует $\delta>0$ такое, что круг $\Delta: \zeta=1$, $|z| \leqslant \delta$ принадлежит $V^{0}$, но окружность $\partial \Delta: \zeta=1,|z|=\delta$ не принадлежит $\overline{f(\mathbb{D})}$. А тогда существуют $b \in($ int $\Delta) \cap \overline{f(\mathbb{D})}$ и многочлен $p(z)$ такие, что $p(z(b))=1$ и $|p|<1$ на $\Delta \cap \overline{f(\mathbb{D})} \backslash b$.

Пусть $r>0$ таково, что бикруг $V:|\zeta-1| \leqslant r,|z| \leqslant \delta$ содержится в $V^{0}$. Обозначим через $X$ пересечение $V$ с замкнутым множеством $\overline{f(\mathbb{D})} \bigcup_{n} \overline{f_{n}(\mathbb{D})}$. Так как $|p|<1$ на компакте $X \cap \partial \Delta$ (возможно, пустом), то существуют $\varepsilon>0, \theta<1$ такие, что $|p|<\theta$ на $X \cap\{|\zeta-1| \leqslant \varepsilon,|z|=\delta\}$. Положим $q=p(z)((1+\zeta) / 2)^{N}$, $N \in \mathbb{N}$. Так как $|(1+\zeta) / 2|<1$ на компакте $X \cap\{|\zeta-1| \geqslant \varepsilon\}$, то $|q|<\theta$ на $X \backslash(V \cap\{|\zeta|=1\}$ при достаточно большом $N$.

Так как $\overline{f_{n}(\mathbb{D})}$ не пересекает множество $V \cap\{|\zeta|=1\}$, то по принципу максимума $|q|<\theta$ на $f_{n}(\mathbb{D}) \cap V$. Так как точка $b \in \overline{f(\mathbb{D})}$ - предельная для $\bigcup f_{n}(\mathbb{D})$, то $|q(b)| \leqslant \theta$, в противоречии с тем, что $q(b)=1$ по построению.

Полученное противоречие показывает, что int $\Delta \subset \overline{f(\mathbb{D})}$, т. е. множество $\overline{f(\mathbb{D})} \cap\left(M_{1} \backslash K_{1}\right)$ открыто. Так как это множество, очевидно, и замкнуто в $M_{1} \backslash K_{1}$, то оно является объединением (возможно, пустым) некоторого семейства связных компонент множества $M_{1} \backslash K_{1}$. Лемма доказана.

ЛЕмма 1.3. Пусть $\widetilde{M} \stackrel{\tilde{\pi}}{\rightarrow} M \rightarrow B$ - голоморфное послойное накрытие трансверсально (вертикально) диск-выпуклого семейства $\pi: M \rightarrow B$. Тогда, если все слои $\widetilde{M}_{\lambda}$ односвязные и некомпактные, то семейство $\pi \circ \tilde{\pi}: \widetilde{M} \rightarrow B$ тоже трансверсально (вертикально) диск-выпуклое.

ДоказатеЛЬСтво. Пусть $U$ - окрестность точки $\lambda \in B$ из определения трансверсальной диск-выпуклости $M, h: \overline{\mathbb{D}} \rightarrow U-$ голоморфный диск и $\widetilde{K}-$ компакт в $(\tilde{\pi} \circ \pi)^{-1}(U)$. Предположим, что существует последовательность замкнутых голоморфных дисков $\left\{\tilde{f}_{n}: \overline{\mathbb{D}} \rightarrow \widetilde{M}\right\}$ таких, что $\pi \circ \tilde{\pi} \circ \tilde{f}_{n}=h$, все $\tilde{f}_{n}(\partial \mathbb{D}) \subset K$, но замыкание объединения любой подпоследовательности образов этих дисков некомпактно. Положим $K=\tilde{\pi}(\widetilde{K})$ и $f_{n}=\tilde{\pi} \circ \tilde{f}_{n}$. Тогда $f_{n}(\partial \mathbb{D}) \subset K$ 
и, по условию, существует компакт $\widehat{K} \subset M$ такой, что $f_{n}(\mathbb{D}) \subset \widehat{K}$. Применяя следствие 1.1 к семейству $\pi^{-1}(h(U \supset \overline{\mathbb{D}}))$ и компакту $X=\widehat{K}$, из семейства $f_{n}$ выделяем подпоследовательность, равномерно сходящуюся внутри $\mathbb{D}$ к некоторому диску $f: \mathbb{D} \rightarrow M$. Чтобы не усложнять обозначений, будем считать, что $f_{n} \rightarrow f$.

Пусть $V$ - окрестность $f(\mathbb{D})$ в $\pi^{-1}(h(\mathbb{D}))$, послойно по себе стягиваемая на $f(\mathbb{D})$ (сечения - топологические круги). Тогда для любого $r \in(0,1)$ найдется номер $n(r)$ такой, что $f_{n}(r \mathbb{D}) \subset V$ для всех $n \geqslant n(r)$. Так как $\widetilde{M}_{\lambda} \rightarrow M_{\lambda}-$ накрытия, то для всякого $\lambda=h(\zeta) \in h(r \mathbb{D})$ существует однозначно определенный диск $\widetilde{V}_{\lambda}$, содержащий точку $\tilde{f}_{n(r)}(\zeta)$ и такой, что $\tilde{\pi}\left(\widetilde{V}_{\lambda}\right)=V_{\lambda}$. Так как $f_{n} \rightarrow f$, то отсюда следует, что $\tilde{f}_{n}$ равномерно на $r \mathbb{D}$ сходятся к некоторому голоморфному диску $\tilde{f}^{r}: r \mathbb{D} \rightarrow \widetilde{M}$ такому, что $\tilde{\pi} \circ \tilde{f}^{r}=f$; при этом диски $\widetilde{V}_{\lambda}$ образуют окрестность $\tilde{f}^{r}(r \mathbb{D})$. При увеличении $r$ диски $\widetilde{V}_{\lambda}$, построенные для меньших его значений, не меняются, поэтому существует голоморфный диск $\tilde{f}: \mathbb{D} \rightarrow M$ такой, что $\tilde{f}_{n} \rightarrow \tilde{f}$ равномерно внутри $\mathbb{D}, \tilde{\pi} \circ \tilde{f}=f$ и $\tilde{f}(\mathbb{D}) \subset \widetilde{V}=\bigcup \widetilde{V}_{h(\zeta)}$.

Предположим, что $\widetilde{K} \cup \overline{\tilde{f}(\mathbb{D})}$ - не компакт. Тогда по лемме 1.2 существует $\zeta \in \partial \mathbb{D}$ такое, что замыкание диска $\tilde{f}(\mathbb{D})$ содержит связную компоненту $D$ с некомпактным замыканием множества $\widetilde{M}_{h(\zeta)} \backslash \widetilde{K}_{h(\zeta)}$. Так как $\widetilde{M}_{\lambda}$ односвязные по условию (т. е. $\widetilde{M}_{\lambda} \rightarrow M_{\lambda}$ - универсальные накрытия), то $\tilde{\pi}(D)=M_{\lambda}$ и, значит, $\overline{f(\mathbb{D})}$ содержит всё $M_{h(\zeta)}$. Но это противоречит тому, что $\overline{f(\mathbb{D})}$ - компакт, а слои $M_{\lambda}$ некомпактные. Таким образом, доказано, что множество $\widetilde{K} \cup \tilde{f}(\mathbb{D})$ компактное, и, ввиду произвольности $\left\{\tilde{f}_{n}\right\}$, трансверсальная диск-выпуклость семейства $\pi \circ \tilde{\pi}: \widetilde{M} \rightarrow B$ доказана.

Пусть теперь $M$ - вертикально диск-выпуклое и $\tilde{f}_{n}: \overline{\mathbb{D}} \rightarrow \widetilde{M}$ - замкнутые голоморфные диски, непрерывные в $\overline{\mathbb{D}}$, вложенные соответственно в слои $\widetilde{M}_{\lambda_{n}}$ и равномерно сходящиеся на $\partial \mathbb{D}, \tilde{f}_{n}(1) \rightarrow a \in \widetilde{M}_{\lambda_{0}}$. Тогда $f_{n}:=\tilde{\pi} \circ \tilde{f}_{n}$ равномерно сходятся к замкнутому голоморфному диску $f: \mathbb{D} \rightarrow M_{\lambda_{0}}$. Так как $\tilde{\pi} \mid \widetilde{M}_{\lambda_{0}} \rightarrow M_{\lambda_{0}}-$ накрытие, то существует однозначно определяемое поднятие $\tilde{f}$ этого диска на $\widetilde{M}_{\lambda_{0}}$ такое, что $\tilde{f}(1)=a$, и, значит, $\tilde{f}_{n} \rightrightarrows \tilde{f}$ на $\partial \mathbb{D}$. По лемме 1.1 , существует послойная биголоморфная тривиализация окрестности $\tilde{f}(\overline{\mathbb{D}})$ в $\widetilde{M}$, содержащая все $\tilde{f}_{n}(\overline{\mathbb{D}})$ при больших $n$. По принципу максимума в этой окрестности, $\tilde{f}_{n} \rightrightarrows \tilde{f}$ в $\overline{\mathbb{D}}$. Лемма доказана.

\section{2. Примеры.}

ПримеР 1.1. Послойное накрытие в общем не является накрытием. Например, пусть $M$ есть область $\left\{(\lambda, w) \in \mathbb{C}^{2}: \lambda w \neq-1\right\}$ с обычной проекцией $(\lambda, w) \mapsto \lambda$. Тогда отображение

$$
\mathbb{C}_{\lambda, z}^{2} \ni(\lambda, z) \mapsto\left(\lambda, \frac{e^{\lambda z}-1}{\lambda}\right) \in M, \quad(0, z) \mapsto(0, z)
$$

является послойным голоморфным накрытием, но прообраз каждой точки $(0, w)$ единственный, а над каждой $(\lambda, w), \lambda \neq 0,-$ счетное число прообразов.

ПримеР 1.2. Простейшие примеры глобально неуниформизуемых семейств это нетривиальные голоморфные линейные расслоения, например стандартное расслоение гиперплоского сечения над $\mathbb{C P}_{1}=\widehat{\mathbb{C}}$ (база односвязная). Локально 
такие семейства униформизуемы просто по определению голоморфных расслоений, и причины глобальной неуниформизуемости во многом чисто топологические. Но топологическая тривиальность базы (например, стягиваемость) в общем тоже ничего не гарантирует, даже для слоений на комплексные плоскости.

Как отмечалось выше, многообразия универсальных накрывающих с голоморфными базовыми сечениями образуют локально гладко тривиальные семейства односвязных поверхностей с глобальными голоморфными сечениями, так называемые косъе иилиндры (см. [35], [37], [52]). Униформизуемость косого цилиндра эквивалентна его голоморфной послойной вложимости в $B \times \widehat{\mathbb{C}}$, что в общем невозможно даже локально.

В качестве примера рассмотрим семейство $\mathbb{C}_{\lambda} \times \mathbb{C}_{w}$ с нестандартной комплексной структурой $J$, относительно которой проекция $(\lambda, w) \mapsto \lambda$ (т. е. функция $\lambda$ ) голоморфна. В качестве базовой формы типа $(1,0)$ относительно $J$ возьмем, кроме $d \lambda$, форму $d w+w^{3} d \bar{\lambda}$. Тогда функции вида $h(\lambda)+2 \bar{\lambda}-1 / w^{2}$, где $h$ голоморфна в $D \subset \mathbb{C}$, являются $J$-голоморфными в области $D \times \mathbb{C}$ и, например, $w /\left(1-2 \bar{\lambda} w^{2}\right)^{1 / 2}$ есть вертикальная координата в окрестности $\mathbb{C} \times 0$. Так как $w \mapsto 2 \bar{\lambda}-1 / w^{2}$ при фиксированном $\lambda$ является двулистным разветвленным накрытием $\mathbb{C} \rightarrow \widehat{\mathbb{C}} \backslash 0$, то слои семейства $\left(\mathbb{C}_{\lambda} \times \mathbb{C}_{w}, J\right)$ конформно эквивалентны $\mathbb{C}$.

Предположим, что это семейство над какой-то областью $D \subset \mathbb{C}_{\lambda}$ униформизуемо, т. е. в $D \times \widehat{\mathbb{C}}_{z}$ (со стандартной структурой и однолистной мероморфной в слоях функцией $z$ ) существует область $\Omega$ вместе с биголоморфизмом $F: \Omega \rightarrow(D \times \mathbb{C}, J)$, сохраняющим проекцию. Тогда $f=z \circ F^{-1}$ - мероморфная функция в $\left(D \times \mathbb{C}_{w}, J\right)$, однолистная в слоях. Так как сужения $d w+w^{3} d \bar{\lambda}$ на $\lambda \times \mathbb{C}$ равны сужениям $d w$, то $f$ - дробно-линейная функция по $w$ при каждом фиксированном $\lambda \in D$. Так как $f$ является $J$-мероморфной функцией, то $d f=f_{\lambda} d \lambda+f_{w}\left(d w+w^{3} d \bar{\lambda}\right)$ вне полюсов $f$, т. е. $f_{\bar{\lambda}}=w^{3} f_{w}$, из чего ясно, что $f$ не может быть линейной по $w$ ни при каком фиксированном $\lambda$. Таким образом, $f(\cdot, w)=a /(w-c)+b$ в $\Omega$ с функциями $a, b, c$, гладкими в $D$, и $f_{\bar{\lambda}}$ должна равняться $w^{3} f_{w}=-a w^{3} /(w-c)^{2}$. Но $f_{\bar{\lambda}}$ ограничена в окрестности $w=\infty$, а $w^{3} f_{w} \rightarrow \infty$ при $w \rightarrow \infty$, и это противоречие показывает, что для любой области $D \subset \mathbb{C}$ семейство $(D \times \mathbb{C}, J) \rightarrow D$ не униформизуемо.

ЗАмЕЧАНИЕ 1.1. Если вместо $w^{3}$ в определении $J$ поставить $w^{2}$, то соответствующее семейство плоскостей, как легко видеть, будет биголоморфно эквивалентным семейству $\left(\mathbb{C}_{\lambda} \times \widehat{\mathbb{C}}_{z}\right) \backslash\{z=\bar{\lambda}\}$ ("мероморфно униформизуемо"), но оно не эквивалентно послойно никакой области в $\mathbb{C}_{\lambda, z}^{2}$ (поскольку тогда соответствующая функция $f$, как выше, линейная, а не квадратичная по $w$ - с тем же противоречием).

ЗАмечание 1.2. Многообразие $(D \times \mathbb{C}, J)$ для любой области $D \subset \mathbb{C}$ не является псевдовыпуклым. В самом деле, пусть $D \supset\left\{\left|\lambda-\lambda_{0}\right| \leqslant r\right\}$. Функции $2\left|\lambda-\lambda_{0}\right|^{2}+\varepsilon^{2}-\left(\lambda-\lambda_{0}\right) / w^{2}, \varepsilon>0, J$-голоморфны в $D \times(\mathbb{C} \backslash 0)$, поэтому поверхности их нулей $S_{\varepsilon}: w^{2}=\left(\lambda-\lambda_{0}\right) /\left(2\left|\lambda-\lambda_{0}\right|^{2}+\varepsilon^{2}\right) J$-голоморфны в $D \times \mathbb{C}$. Граница поверхности $S_{\varepsilon} \cap\left\{\left|\lambda-\lambda_{0}\right|<r\right\}$ при любом $\varepsilon>0$ лежит в компакте $\left\{\left|\lambda-\lambda_{0}\right|=r,|w| \leqslant 1 / r\right\}$, а точки $\left(\lambda_{0}+\varepsilon, 1 / \sqrt{3 \varepsilon}\right) \in S_{\varepsilon}$ стремятся к $\left(\lambda_{0}, \infty\right)$ 
при $\varepsilon \rightarrow 0$, что противоречит псевдовыпуклости. В частности, $(D \times \mathbb{C}, J)$ не являются многообразиями Штейна.

В приложениях, связанных с голоморфными слоениями на многообразиях Штейна, многообразия универсальных накрывающих (соответствующие штейновым сечениям) сами являются многообразиями Штейна (см. [35], [36]). Поэтому естественно изучить униформизуемость косых цилиндров с этим дополнительным свойством.

Первый пример неуниформизуемого косого цилиндра Штейна построил А. Глуцюк [28], который показал также [29], что в качестве такого цилиндра можно взять многообразие универсальных накрывающих некоторого алгебраического слоения на аффинной алгебраической комплексно двумерной поверхности без особенностей, причем соответствующее семейство не униформизуемо ни над какой областью в базе. Примеры довольно сложные и мы здесь ограничимся ссылками.

ПримеР 1.3. Из трансверсальной плюс вертикальной диск-выпуклости голоморфного семейства не следует обычная диск-выпуклость пространства этого семейства. В самом деле, область $M=\mathbb{C}^{2} \backslash(0,0)$ является пространством семейства римановых поверхностей $\pi: M \rightarrow \mathbb{C}$ с $\pi(z)=z_{2}^{2}-z_{1}^{3}$. Сама $M$ очевидно не диск-выпуклая.

С другой стороны, пусть $h: \overline{\mathbb{D}} \rightarrow \mathbb{C}$ - голоморфный вложенный диск, $h(0)=0$, $K$ - компакт в $\pi^{-1}(h(\partial \mathbb{D}))$ и $f_{n}: \mathbb{D} \rightarrow M$ - голоморфные диски такие, что $\pi \circ f_{n}=h$ и множества $K \cup f_{n}(\mathbb{D})$ компактные. Тогда можно считать, что $f_{n}$ сходятся к некоторому $f: \mathbb{D} \rightarrow \mathbb{C}^{2}$ равномерно внутри $\mathbb{D}$ и $\pi \circ f=h$.

Предположим, что $f(0)=(0,0)$. Тогда $f(\zeta)=\left(z_{1}(\zeta), z_{2}(\zeta)\right)$, обе функции $z_{j}(\zeta)$ равны нулю при $\zeta=0$, и, значит, функция $z_{2}^{2}(\zeta)-z_{1}^{3}(\zeta)$ имеет в 0 нуль не менее чем второго порядка. Но по условию $\pi \circ f=h-$ вложение, т. е. нуль должен быть простым. Это противоречие показывает, что семейство $\pi: M \rightarrow \mathbb{C}$ трансверсально диск-выпуклое.

Пусть теперь $f_{n}: \overline{\mathbb{D}} \rightarrow M$ - замкнутые вложенные вертикальные голоморфные диски, $f_{n}(\overline{\mathbb{D}}) \subset S_{n}=\left\{z_{2}^{2}-z_{1}^{3}=c_{n}\right\}$ для некоторой последовательности $c_{n} \rightarrow 0$ и $f_{n} \rightrightarrows f$ в $\overline{\mathbb{D}}, f(\partial \mathbb{D}) \subset M$. Предположим, что $f(\mathbb{D}) \ni(0,0)$. Тогда $f(\overline{\mathbb{D}}) \subset S_{0}: z_{2}^{2}=z_{1}^{3}$ и поэтому существует $\delta>0$ такое, что $f(\mathbb{D}) \supset S \cap\left\{\left|z_{1}\right| \leqslant \delta\right\}$. Но тогда $f_{n}(\mathbb{D})$ при достаточно больших $n$ содержат окружности $S_{n} \cap\left\{\left|z_{1}\right|=\delta\right\}$. Так как $f_{n}: \mathbb{D} \rightarrow S_{n}$ - вложения и любая замкнутая жорданова кривая в $\mathbb{D}$ ограничивает односвязную область, то $f_{n}(\mathbb{D}) \cap\left\{\left|z_{1}\right|<\delta\right\}$ должны быть топологическими кругами. С другой стороны, при большом $n, n>n(\delta)$, поверхность $S_{n} \cap\left\{\left|z_{1}\right|<\delta\right\}$ есть тор без топологического круга, с нетривиальным циклом $S_{n} \cap\left\{z_{1} \in \gamma\right\}$, где $\gamma$ есть, например, отрезок, соединяющий точки $c_{n}^{1 / 3} e^{ \pm i \pi / 3}$ (цикл, исчезающий при $n \rightarrow \infty)$. Это противоречие показывает, что $f(\overline{\mathbb{D}}) \subset M$, и, значит, семейство $\pi: M \rightarrow \mathbb{C}$ является вертикально диск-выпуклым.

Аналогично можно доказать, что для любого многочлена $p$ в $\mathbb{C}^{2}$ с неприводимыми уровнями и конечным критическим множеством $\sigma$ семейство $p$ : $\mathbb{C}^{2} \backslash \sigma \rightarrow \mathbb{C}$ является трансверсально и вертикально диск-выпуклым (трансверсальная диск-выпуклость следует, как выше, из принципа аргумента, а вертикальная - из наличия исчезающих циклов в окрестности каждой критической точки). 
1.3. Сферы и торы. Голоморфные семейства сфер локально тривиальны. Поскольку все сферы биголоморфно эквивалентные, то это утверждение является простым частным случаем теоремы Грауэрта-Фишера [23]. Мы приведем здесь другое доказательство, в основном следуя [37].

Пусть $\pi: M \rightarrow B$ - голоморфное семейство над произвольным комплексным банаховым многообразием $B$, слои $M_{\lambda}$ которого конформно эквивалентны сфеpe Римана $\widehat{\mathbb{C}}$. Предположим, что есть три глобальных голоморфных сечения $s_{0}, s_{1}, s_{\infty}$ проекции $\pi$ с попарно непересекающимися образами. Тогда в каждом слое $M_{\lambda}$ однозначно определена мероморфная функция, равная $j$ в точках $s_{j}(\lambda)$, соответственно. Покажем, что эта функция мероморфна на $M$ (хотя даже непрерывность ее не очевидна). Доказательство разобьем на две части.

Шаг $1 . B=\mathbb{D}$. В этом случае (как и в случае любого конечномерного $B$ ) $\pi: M \rightarrow B$ есть гладкое расслоение по теореме Эресмана [22]. Так как у нас есть три глобальных сечения, то $M$ послойно диффеоморфно $B \times \widehat{\mathbb{C}}$, причем диффеоморфизм $\phi: B \times \widehat{\mathbb{C}} \rightarrow M$ можно выбрать так, что он сохраняет ориентацию и $\phi(\lambda, j)=s_{j}(\lambda), j=0,1, \infty$ (на $\widehat{\mathbb{C}}$ фиксируем глобальную мероморфную координату $w)$. Комплексная структура на $M$ при этом индуцирует на $B \times \widehat{\mathbb{C}}$ комплексную структуру $J$, в общем отличную от стандартной. Так как диффеоморфизм $\phi$ послойный, то $\lambda$ остается голоморфной функцией и, значит, $d \lambda$ - дифференциал типа $(1,0)_{J}$ (т. е. типа $(1,0)$ относительно структуры $\left.J\right)$.

Слои $\lambda \times \widehat{\mathbb{C}}$ голоморфны относительно $J$, и сужение $J$ на такой слой однозначно определяется функцией $\mu(\lambda, \cdot) \in L^{\infty}(\mathbb{C})$ такой, что $d w+\mu(\lambda, w) d \bar{w}-$

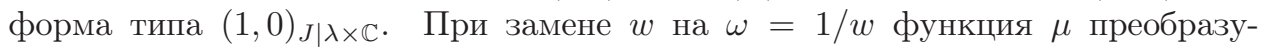
ется в $\mu(\lambda, 1 / \omega) \omega^{2} / \bar{\omega}^{2}$. Так как $\phi$ сохраняет ориентацию и $\widehat{\mathbb{C}}-$ компакт, то $|\mu(\lambda, \cdot)| \leqslant k(\lambda)<1$ с некоторой непрерывной функцией $k$. Так как $\phi-$ диффеоморфизм, то функция $\mu(\lambda, w)$ гладкая и все ее частные производные по $\lambda, \bar{\lambda}$ тоже гладкие в $\mathbb{D} \times \mathbb{C}$ и равномерно ограниченные, поскольку структура $J$ гладкая на $\mathbb{D} \times \widehat{\mathbb{C}}($ см. преобразование $\mu$ при замене $w$ на $1 / w)$. Таким образом, отображение $B \ni \lambda \mapsto \mu(\lambda, \cdot) \in L^{\infty}(\mathbb{C})$ гладкое. А тогда, по теореме Альфорса-Берса [1], [2], для всякой фиксированной $\lambda \in B$ существует единственное гомеоморфное в $\mathbb{C}$ решение $z(\lambda, w)$ уравнения Бельтрами $z_{\bar{w}}=\mu(\lambda, \cdot) z_{w}$, нормированное условиями $z(\lambda, 0)=0, z(\lambda, 1)=1$, причем функция $z$ гладкая на $\mathbb{D} \times \mathbb{C}$.

Так как $d z=z_{w}(d w+\mu(\lambda, \cdot) d \bar{w})$ на $\lambda \times \mathbb{C}$, то $z$ является $J$-голоморфной функцией на каждом слое и поэтому $\bar{\partial}_{J} z$, т. е. компонента $d z$ типа $(0,1)_{J}$, уже на всем $\mathbb{D} \times \mathbb{C}$ имеет вид $a d \bar{\lambda}$ с гладкой функцией $a=\partial z(\lambda, w) / \partial \bar{\lambda}$, которую мы далее рассматриваем как функцию от $\lambda, z$. Таким образом, формы $d \lambda$ и $\partial_{J} z=$ $d z-a d \bar{\lambda}$ образуют на $\mathbb{D} \times \mathbb{C}_{z}$ базис форм типа $(1,0)_{J}$.

Так как $J$ - комплексная структура (т. е. в каждой точке есть локальные $J$-голоморфные координаты), то форма

$$
d \partial_{J} z=\bar{\partial}_{J} \partial_{J} z=-\left(a_{z} d z+a_{\bar{z}} d \bar{z}+a_{\lambda} d \lambda\right) \wedge d \bar{\lambda}
$$

имеет нулевую компоненту типа $(0,2)_{J}$. Так как $d z \wedge d \bar{\lambda}=\partial_{J} z \wedge d \bar{\lambda}$ и $d \lambda \wedge d \bar{\lambda}-$ формы типа $(1,1)_{J}$, а

$$
d \bar{z} \wedge d \bar{\lambda}=(d \bar{z}-\bar{a} d \lambda) \wedge d \bar{\lambda}+\bar{a} d \lambda \wedge d \bar{\lambda}
$$

имеет ненулевую $(0,2){ }_{J}$-компоненту, то отсюда следует, что $a_{\bar{z}} \equiv 0$, т. е. функция $a(\lambda, z)$ голоморфна по $z$ (в обычном смысле). 
Так как $J$ - структура на $\mathbb{D} \times \widehat{\mathbb{C}}$ и функция $a$ определяется по $J$ однозначно, то в результате замены $z \mapsto \zeta=1 / z$ базисом $(1,0)_{J}$-форм на $\mathbb{D} \times(\mathbb{C} \backslash 0)$ станут $d \lambda$ и $d \zeta+\zeta^{2} a(\lambda, 1 / \zeta) d \bar{\lambda}$, причем функция $\zeta^{2} a(\lambda, 1 / \zeta)$ должна (вместе с $\left.J\right)$ гладким образом продолжаться на все $\mathbb{D} \times \mathbb{C}_{\zeta}$. Так как $a(\lambda, z)$ голоморфна по $z$, то из этого следует, что $a$ есть многочлен по $z$ степени не выше 2 . Так как $\mathbb{D} \times \infty_{z}-$ голоморфный диск относительно $J$, соответствующий $\zeta=0$ (см. нормировки $\phi$ и $z)$, то сужение на него (продолженной) $(1,0)_{J}$-формы $d \zeta+\zeta^{2} a(\lambda, 1 / \zeta) d \bar{\lambda}$ должно быть пропорционально $d \lambda$. Из этого очевидно следует, что $a-$ многочлен по $z$ степени не выше $1, a(\lambda, z)=a_{0}(\lambda)+a_{1}(\lambda) z$. Так как $\mathbb{D} \times 0$ - тоже $J$-голоморфный диск и сужение на него $\partial_{J} z$ равно $-a_{0}(\lambda) d \bar{\lambda}$, то $a_{0} \equiv 0$. Наконец, диск $\mathbb{D} \times 1$ тоже $J$-голоморфный, сужение $\partial_{J} z$ на него равно $-a_{1}(\lambda) d \bar{\lambda}$ и, значит, $a_{1} \equiv 0$. Таким образом, $a \equiv 0$ и мы доказали, что $d z-$ форма типа $(1,0)_{J}$, т. е. функция $z(\lambda, w) J$-голоморфна на $\mathbb{D} \times \mathbb{C}$ и $J$-мероморфна $\mathbb{D} \times \widehat{\mathbb{C}}$.

Вывод: в случае $B=\mathbb{D}$ функция $z \circ \phi^{-1}$, о которой речь шла в самом начале этого пункта, мероморфна на $M$ и, значит, отображение $M \ni p \mapsto$ $\left(\pi(p), z \circ \phi^{-1}(p)\right) \in \mathbb{D} \times \widehat{\mathbb{C}}$ биголоморфно.

Шаг 2. Пусть теперь $B$ - произвольное комплексное банахово многообразие и $z$ - функция на $M$, мероморфная и однолистная на каждом слое $M_{\lambda}$ и равная $j$ в соответствующих точках $s_{j}(\lambda), j=0,1, \infty$ (нормированная мероморфная координата в слоях). Пусть $f: \mathbb{D} \rightarrow M-$ произвольный голоморфный диск, не пересекающий $s_{\infty}(B)$, и $h:=\pi \circ f$. Тогда для диска $h: \mathbb{D} \rightarrow B$ определено голоморфное семейство сфер $\pi^{\prime}: M^{\prime} \rightarrow \mathbb{D}$, прообраз семейства $\pi: M \rightarrow B$ относительно отображения $h$. По доказанному выше, прообраз на $M^{\prime}$ функции $z$ является мероморфной функцией, в частности, функция $z \circ f$ голоморфна в $\mathbb{D}$. Ввиду произвольности $f$ и локальной ограниченности $z$ вне $s_{\infty}(B)$, функция $z$ голоморфна на $M \backslash s_{\infty}(B)$. Аналогично, $1 / z$ голоморфна на $M \backslash s_{0}(B)$ и, значит, $z$ мероморфна на $M$, а отображение $M \ni p \mapsto(\pi(p), z(p)) \in B \times \widehat{\mathbb{C}}$ является послойным биголоморфизмом.

Опишем теперь общие голоморфные семейства сфер.

Теорема 1.1. Всякое голоморфное семейство сфер $\pi: M \rightarrow B$ над произвольным комплексным банаховым многообразием $B$ является локально голоморфно тривиальным расслоением с послойно дробно-линейными отображениями переходов.

Если $\pi$ допускает глобальное голоморфное сечение, то $M$ есть послойное замыкание голоморфного расслоения комплексных плоскостей с послойно аффинными функииями переходов.

Если $\pi$ допускает два глобальных голоморфных сечения с непересекающимися образами, то $M$ есть послойное замыкание голоморфного линейного расслоения.

Наконеи, если $\pi$ допускает три глобальных голоморфных сечения с попарно не пересекающимися образами, то семейство $\pi: M \rightarrow B$ тривиально, т.е. биголоморфно эквивалентно семейству $B \times \widehat{\mathbb{C}} \rightarrow B$.

ДокАЗАТЕЛЬСтво. Если $s$ - глобальное голоморфное сечение $\pi$, то для всякой $\alpha \in B$ существуют окрестность $V_{\alpha}$ и мероморфная однолистная в слоях 
функция $z_{\alpha}$ в $U_{\alpha}:=\pi^{-1}\left(V_{\alpha}\right)$, равная $\infty$ в точках $s(\lambda), \lambda \in V_{\alpha}$. В попарных пересечениях $U_{\alpha} \cap U_{\beta}$ тогда $z_{\beta}=a_{\beta \alpha} z_{\beta}+b_{\beta \alpha}$ с голоморфными коэффициентами, постоянными в слоях.

Если $s_{0}, s_{\infty}$ - не пересекающиеся глобальные голоморфные сечения, то указанные функции $z_{\alpha}$ можно выбрать так, что $z_{\alpha}\left(s_{0}(\lambda)\right)=0, z_{\alpha}\left(s_{\infty}(\lambda)\right)=\infty$, $\lambda \in V_{\alpha}$. Тогда в $U_{\alpha} \cap U_{\beta}$ имеем $z_{\beta}=a_{\beta \alpha} z_{\alpha}$, функции $a_{\alpha \beta}$, постоянные на слоях $M_{\lambda}$, образуют мультипликативный 1-коцикл на $B$ (соответствующий покрытию $\left.\left\{V_{\alpha}\right\}\right)$ и, таким образом, определяют на $B$ голоморфное линейное расслоение со слоями $M_{\lambda} \backslash s_{\infty}(\lambda)$. Теорема доказана.

И как следствие - достаточные условия тривиальности (= униформизуемости в этом случае).

СледСтвиЕ 1.2. Голоморфное семейство сфер $\pi: M \rightarrow B$ тривиально, если выполняется одно из следующих условий:

1) $\pi$ допускает глобальное голоморфное сечение и на В разрешимы первая и вторая проблемы Кузена;

2) $\pi$ допускает два глобальных голоморфных сечения с непересекающимися образами и на В разрешима вторая проблема Кузена;

3) $\pi$ допускает три глобальных голоморфных сечения с попарно непересекаюшимися образами.

ДокАЗАтельство. Условие 2) достаточно потому, что тогда всякое голоморфное линейное расслоение на $B$ тривиально, а значит, тривиально и семейство сфер, которое получается из него добавлением сечения $s_{\infty}(B)$.

Предположим теперь, что выполнено условие 1$), z_{\alpha}$ - мероморфные функции в $U_{\alpha}$ и, как выше, $z_{\beta}=a_{\beta \alpha} z_{\alpha}+b_{\beta \alpha}$. Тогда $a_{\alpha \beta}=1 / a_{\beta \alpha}$ и $z_{\gamma}=a_{\gamma \alpha} a_{\alpha \beta} z_{\beta}+$ $\left(b_{\gamma \alpha}-b_{\beta \alpha} a_{\alpha \beta}\right)$, откуда $a_{\gamma \beta} a_{\beta \alpha} a_{\alpha \gamma}=1$ в $U_{\alpha} \cap U_{\beta} \cap U_{\gamma}$. Таким образом, функции $a_{\beta \alpha}$ образуют мультипликативный 1 -коцикл на $B$, соответствующий покрытию $\left\{V_{\alpha}:=\pi\left(U_{\alpha}\right)\right\}$. Ввиду разрешимости второй проблемы Кузена на $B$, существуют голоморфные функции $c_{\alpha}$, постоянные на слоях $M_{\lambda}, \lambda \in V_{\alpha}$, нигде не равные нулю в $U_{\alpha}$ и такие, что $a_{\beta \alpha}=c_{\alpha} / c_{\beta}$ в $U_{\alpha} \cap U_{\beta}$. Положим $w_{\alpha}=c_{\alpha} z_{\alpha}$ в $U_{\alpha}$. Тогда $w_{\beta}=c_{\beta} a_{\beta \alpha} z_{\alpha}+c_{\beta} b_{\beta \alpha}=w_{\alpha}+b_{\beta \alpha}^{*}$ и функции $b_{\beta \alpha}^{*}$ очевидно образуют аддитивный 1-коцикл на покрытии $\left\{V_{\alpha}\right\}$. Так как на $B$ по условию разрешима первая проблема Кузена, то существуют голоморфные функции $h_{\alpha}$ в $U_{\alpha}$, постоянные на слоях и такие, что $b_{\beta \alpha}^{*}=h_{\alpha}-h_{\beta}$. А тогда голоморфные функции $z_{\alpha}^{*}:=w_{\alpha}+h_{\alpha}$ совпадают в попарных пересечениях, функция $z:=\left\{z_{\alpha}^{*}\right.$ в $\left.U_{\alpha}\right\}$ мероморфна на $M$ и отображение $p \longmapsto(\pi(p), z(p))$ является послойным биголоморфизмом $M$ на $B \times \widehat{\mathbb{C}}$. Следствие доказано.

Из доказательства видно, что точность (разрешимость) обоих построенных в нем коциклов является необходимым условием тривиальности, в частности, если на $B$ не разрешима первая или вторая проблема Кузена, то над $B$ есть нетривиальные голоморфные семейства сфер с одним или двумя глобальными голоморфными сечениями.

Различные комплексные торы в общем случае конформно не эквивалентны, есть модули, однако послойные универсальные накрывающие голоморфных семейств торов также локально тривиальны, как и в случае сфер. 
Теорема 1.2. Пусть $\pi: M \rightarrow B$ - голоморфное семейство торов над комплексным банаховым многообразием. Если т допускает глобальное голоморфное сечение и $\tilde{\pi}: \widetilde{M} \rightarrow M-$ соответствующее ему послойно универсальное накрытие, то $\pi \circ \tilde{\pi}: \widetilde{M} \rightarrow B$ биголоморфно эквивалентно семейству $B \times \mathbb{C} \rightarrow B$.

ДокАзАтельство. Как и выше, основным здесь является случай $B=\mathbb{D}$ с глобальным голоморфным сечением $s: \mathbb{D} \rightarrow M$.

Фиксируем универсальное накрытие $\phi_{0}: \mathbb{C} \rightarrow M_{0}$ с координатой $w$ в $\mathbb{C}$, однозначно определяемой условием $\phi_{0}(0)=\phi_{0}(1)=s(0)$. Тогда тор $M_{0}$ конформно эквивалентен $\mathbb{C} / \Gamma_{0}$, где $\Gamma_{0}$ - решетка, содержащая 0,1 (группу сдвигов по этой решетке обозначим так же; недоразумений не будет).

Опять по теореме Эресмана [22], существует сохраняющий ориентацию послойный диффеоморфизм $\mathbb{D} \times M_{0} \rightarrow M$, который можно выбирать так, что $\lambda \times s(0) \mapsto s(\lambda), \lambda \in \mathbb{D}$. Он индуцирует послойный диффеоморфизм $\tilde{\phi}: \mathbb{D} \times \mathbb{C} \rightarrow$ $\widetilde{M}$ с $\tilde{\phi}(0, \cdot)=\phi_{0}$.

Обозначим через $J$ прообраз в $\mathbb{D} \times \mathbb{C}$ комплексной структуры на $\widetilde{M}$ относительно отображения $\tilde{\phi}$. Тогда, по построению, $J$ инвариантна относительно действия в $\mathbb{D} \times \mathbb{C}$ группы $\Gamma_{0}$. Как и выше, сужение $J$ на слой $\lambda \times \mathbb{C}$ определяется формой $d w+\mu(\lambda, \cdot) d \bar{w}$ типа $(1,0)_{J \mid \lambda \times \mathbb{C}}$ с функцией $\mu$, гладкой на $\mathbb{D} \times \mathbb{C},|\mu|<1$. Так как $J$ инвариантна относительно $\Gamma_{0}$ и $\mu$ по $J$ определяется однозначно, то $\mu(\lambda, w+c) \equiv \mu(\lambda, w)$ для всех $c$ из решетки $\Gamma_{0}$. Из этого очевидно следует, что каждая частная производная $\mu$ по $\lambda, \bar{\lambda}$ равномерно ограничена в $\mathbb{C}$, т. е. отображение $\mathbb{D} \ni \lambda \mapsto \mu(\lambda, \cdot) \in L^{\infty}(\mathbb{C})$ гладкое.

По теореме Альфорса-Берса [1], [2], для всякой $\lambda \in \mathbb{D}$ существует единственное гомеоморфное в $\mathbb{C}$ решение $z(\lambda, \cdot)$ уравнения Бельтрами $z_{\bar{w}}=\mu(\lambda, \cdot) z_{w}$, нормированное условиями $z(\lambda, 0) \equiv 0, z(\lambda, 1) \equiv 1$ и гладкое по совокупности переменных $\lambda, w$.

Относительно координат $\lambda, z$ в $\mathbb{D} \times \mathbb{C}$ структура $J$ имеет $(1,0)_{J}$-базис $d \lambda$, $\partial_{J} z=d z-a(\lambda, z) d \bar{\lambda}$ с гладкой функцией $a$. Так как структура $J-$ комплексная (= интегрируемая почти комплексная), то функция $a$ голоморфна по $z$ (см. выше шаг 1).

Так как $\mu$ инвариантна относительно сдвигов по Г 0 то, ввиду единственности решения,

$$
z(\lambda, w)=\frac{z(\lambda, w+c)-z(\lambda, c)}{z(\lambda, 1+c)-z(\lambda, c)}
$$

для любых $c \in \Gamma_{0}$. Таким образом, сдвигам $w \mapsto w+c, c \in \Gamma_{0}$, соответствуют аффинные преобразования

$$
z \mapsto(z(\lambda, 1+c)-z(\lambda, c)) z-z(\lambda, c)=: A(\lambda, c) z+B(\lambda, c) .
$$

При каждом фиксированном $\lambda \in \mathbb{D}$ они образуют дискретную группу $\Gamma_{\lambda}^{\prime} \subset$ Aut $\mathbb{C}_{z}$, изоморфную $\Gamma_{0}$, элементы которой не имеют неподвижных точек. Из последнего следует, что $A(\lambda, c) \equiv 1$, т. е. $\Gamma_{\lambda}^{\prime}-$ группа сдвигов по решетке $\Gamma_{\lambda}^{\prime} \ni 0$ с образующими $1=z(\lambda, 1)$ и некоторой $\tau(\lambda)$.

Структура $J$ инвариантна относительно таких преобразований координаты $z$, т. е. формы $d(z+B(\lambda))-a(\lambda, z+B(\lambda)) d \bar{\lambda}, B(\lambda)=n+m \tau(\lambda), n, m \in \mathbb{Z}$, должны иметь тип $(1,0)_{J}$. Так как $d(z+B)=d z+B_{\bar{\lambda}} d \bar{\lambda}+B_{\lambda} d \lambda$, то это значит, что $a(\cdot, z+B)=a(\cdot, z)+B_{\bar{\lambda}}$. Отсюда $a_{z}(\lambda, z+n+m \tau) \equiv a_{z}(\lambda, z)$ для 
всех $n, m \in \mathbb{Z}$ и, значит, функции $a_{z}(\lambda, \cdot)$ равномерно ограниченные. Так как $a$ голоморфна по $z$, то из этого и теоремы Лиувилля следует, что она аффинная по $z$, т. е. $a(\cdot, z)=a_{0}+a_{1} z$.

Так как график $z=0$ является $J$-голоморфным и сужение на него формы $\partial_{J} z$ равно $-a_{0}(\lambda) d \bar{\lambda}$, то $a_{0} \equiv 0$. Аналогично, из $J$-голоморфности графика $z=1$ следует, что $a_{1} \equiv 0$ и, значит, $a \equiv 0$.

Таким образом, функция $z$ является $J$-голоморфной на $\mathbb{D} \times \mathbb{C}$, значит, $z \circ \phi^{-1}$ голоморфна на $\widetilde{M}$ и отображение $p \mapsto\left(\pi \circ \tilde{\pi}(p), z \circ \phi^{-1}(p)\right)$ есть послойный биголоморфизм семейства $\pi \circ \tilde{\pi}: \widetilde{M} \rightarrow \mathbb{D}$ на $\mathbb{D} \times \mathbb{C} \rightarrow \mathbb{D}$.

Случай общей базы $B$ при наличии глобального сечения $s: B \rightarrow M$ сводится к доказанному выше. Фиксируем $\lambda_{0} \in B$, универсальное накрытие $\tilde{\pi}_{0}: \mathbb{C} \rightarrow$ $M_{\lambda_{0}}$ и выберем аффинную координату $w$ на $\mathbb{C}$ так, что точки $w=0, w=1$ проектируются в $s\left(\lambda_{0}\right)$ и 1 - одна из образующих решетки $\Gamma_{0}=\tilde{\pi}_{0}^{-1}\left(s\left(\lambda_{0}\right)\right)$. Обозначим через $s_{0}, s_{1}$ непрерывные сечения проекции $\pi \circ \tilde{\pi}$ такие, что $\tilde{\pi}\left(s_{j}\right)=s$ и $w\left(s_{0}\left(\lambda_{0}\right)\right)=0, w\left(s_{1}\left(\lambda_{0}\right)\right)=1$. Тогда в каждом слое $\widetilde{M}_{\lambda}$ однозначно определена голоморфная координата $z(\lambda, \cdot)$ такая, что $z\left(\lambda, s_{j}(\lambda)\right) \equiv j, j=0,1$, и остается показать, что $z$ голоморфна не только вдоль слоев, но и на всем $\widetilde{M}$.

Пусть $f: \mathbb{D} \rightarrow \widetilde{M}$ - голоморфный диск и $h=\pi \circ \tilde{\pi} \circ f$. Тогда $h: \mathbb{D} \rightarrow B$ голоморфно и над $\mathbb{D}$ определен прообраз $\pi^{\prime}: \widetilde{M^{\prime}} \rightarrow \mathbb{D}$ семейства $\widetilde{M} \rightarrow B$ относительно $h$, причем это $\pi^{\prime}$ имеет глобальное голоморфное сечение, которое является прообразом сечения $s_{0}$. Прообраз функции $z$ на $\widetilde{M}^{\prime}$ обозначим через $z^{\prime}$, а прообраз диска $f$ - через $f^{\prime}$. По доказанному выше, функция $z^{\prime}$ голоморфна на $\widetilde{M}^{\prime}$, в частности, функция $z^{\prime} \circ f^{\prime}$ голоморфна в $\mathbb{D}$, а это эквивалентно тому, что функция $z \circ f$ голоморфна в $\mathbb{D}$. Таким образом, $z$ - локально ограниченная на $\widetilde{M}$ функция, голоморфная на каждом голоморфном диске в $\widetilde{M}$ и, следовательно, голоморфная.

Первое утверждение теоремы вытекает из доказанного и того, что локально голоморфные сечения $\pi$ существуют. Теорема доказана.

ЗАмЕчАниЕ 1.3. Как видно из доказательства (случай $B=\mathbb{D}$ ), сдвиги из групп $\Gamma_{\lambda}^{\prime}$ голоморфны по $\lambda$ (поскольку $\left.a \equiv 0\right)$ и многообразие $M$ является фактором комплексного многообразия $(\mathbb{D} \times \mathbb{C}, J)$ по действию группы $\Gamma^{\prime}$, послойно действующей как $\Gamma_{\lambda}^{\prime}$. Однако в общем случае не существует биголоморфных преобразований $\lambda \times \mathbb{C}$ в $\lambda^{\prime} \times \mathbb{C}, \lambda^{\prime} \neq \lambda$, коммутирующих с действиями этой группы в слоях, т.е. в общем случае семейства торов не являются локально голоморфно тривиальными.

Например, если $\lambda$ лежит в верхней полуплоскости $\mathbb{H}$ и $M_{\lambda}$ есть фактор $\mathbb{C}$ по решетке $\Gamma_{\lambda} \ni 0$ с образующими $1, \lambda$, то фактор $\mathbb{H} \times \mathbb{C}$ по группе голоморфных автоморфизмов, действующих послойно как $\Gamma_{\lambda}$, дает голоморфное семейство торов над $\mathbb{H}$. Но решетки с образующими $1, \lambda$ и $1, \lambda^{\prime}$ при близких $\lambda \neq \lambda^{\prime}$ можно перевести друг в друга лишь $\mathbb{R}$-линейным преобразованием, но никаким $\mathbb{C}$-аффинным преобразованием этого сделать нельзя.

С другой стороны, бывают и небезынтересные локально голоморфно тривиальные семейства торов. Например, пусть $M^{0}=\mathbb{C}^{2} \backslash\left\{z_{2}^{2}=z_{1}^{3}\right\}, \pi(z)=z_{2}^{2}-z_{1}^{3}$ и $M_{\lambda}$ - пополнение $M_{\lambda}^{0}$ точкой на бесконечности. Тогда в таким образом послойно пополненном множестве $M \supset M^{0}$ есть комплексная структура, совпадающая с таковой на $M^{0}$, и проекция $\pi: M_{\lambda} \rightarrow \lambda$ превращает $M$ в голоморфное 
семейство торов. Отображение $M_{\lambda} \rightarrow M_{1}$ по локально определенной формуле $\left(z_{1}, z_{2}\right) \mapsto\left(z_{1} \lambda^{-1 / 3}, z_{2} \lambda^{-1 / 2}\right)$ является биголоморфизмом и, значит, семейство $\pi: M \rightarrow \mathbb{C} \backslash 0$ локально голоморфно тривиальное.

СлеДСтвие 1.3. Семейство торов $\pi: M \rightarrow B$ униформизуемо, если выполнено одно из следующих условий:

1) на В разрешимы первая и вторая проблемы Кузена;

2) $\pi$ допускает глобальное голоморфное сечение.

ДокАзАтельство. Случай 2) разобран выше. Доказательство в случае 1) такое же, как в случае 1) следствия 1.2.

Как и в случае сфер, неразрешимость на $B$ одной из проблем Кузена (как, например, для $B=\mathbb{C P}_{n}, n \geqslant 1$ ) гарантирует существование над $B$ неуниформизуемых голоморфных семейств торов.

При наличии глобального непрерывного сечения проекции $\pi$ условия 1$)$ можно слегка ослабить (по крайней мере, в случае конечномерной базы $B$ ), а именно оставить лишь условие разрешимости на $B$ первой проблемы Кузена. В самом деле, комплексное многообразие $\widetilde{M}$, соответствующее этому непрерывному сечению, является голоморфным семейством комплексных плоскостей, локально голоморфно тривиальным (поскольку над малыми координатными шарами в $B$ голоморфные сечения $\pi$ существуют), с функциями переходов, аффинными в слоях и голоморфными по $\lambda \in V_{\alpha} \cap V_{\beta}, z_{\beta}=a_{\beta \alpha}(\lambda) z_{\alpha}+b_{\beta \alpha}(\lambda)$. Так как есть глобальное непрерывное сечение $\pi$, то на $\widetilde{M}$ есть глобальная непрерывная функция $w$, голоморфная и однолистная на каждом слое $\lambda \times \mathbb{C}$. А тогда $w=\tilde{a}_{\alpha} z_{\alpha}+\tilde{b}_{\alpha}$ с непрерывными $\tilde{a}_{\alpha}, \tilde{b}_{\alpha}$ в $V_{\alpha}$ и, значит, $a_{\beta \alpha}=\tilde{a}_{\alpha} / \tilde{a}_{\beta}$. Измельчая покрытие, можно считать, что множества $V_{\alpha}$ связные и односвязные и потому существуют непрерывные в $V_{\alpha}$ логарифмы $\log \tilde{a}_{\alpha}$. А тогда функции $\log a_{\beta \alpha}:=\log \tilde{a}_{\alpha}-\log \tilde{a}_{\beta}$ образуют на $B$ аддитивный голоморфный 1-коцикл. Если первая проблема Кузена на $B$ разрешима, то $\log a_{\beta \alpha}=c_{\beta}-c_{\alpha}$ для некоторых голоморфных соответственно в $V_{\alpha}, V_{\beta}$ функций $c_{\alpha}, c_{\beta}$ и, заменяя $z_{\alpha}$ на $z_{\alpha}^{*}=e^{c_{\alpha}} z_{\alpha}$, мы получаем соотношения переходов $z_{\beta}^{*}=z_{\alpha}^{*}+b_{\beta \alpha}^{*}$, опять подправляемые решениями соответствующей первой проблемы до голоморфных функций в $U_{\alpha}=\pi^{-1}\left(V_{\alpha}\right)$, аффинных в слоях и уже совпадающих на попарных пересечениях.

Существование глобального непрерывного сечения, без дополнительных “комплексных" условий на базу, недостаточно для униформизуемости семейства торов.

ПримеР 1.4. Пусть $B=\mathbb{C}^{2} \backslash(0,0), V_{j}=B \cap\left\{\lambda_{j} \neq 0\right\}$ и $U_{j}=V_{j} \times \mathbb{C}_{z_{j}}$. Склеивая комплексные карты $U_{j}$ отождествлением $z_{2} \equiv z_{1}+1 /\left(\lambda_{1} \lambda_{2}\right)$ на $U_{1} \cap U_{2}$, мы получаем комплексное многообразие $\widetilde{M}$ с голоморфной проекцией $\tilde{\pi}: \widetilde{M} \rightarrow B$. Сечение $\tilde{\pi}$ - это пара функций $f_{j}: V_{j} \rightarrow \mathbb{C}$ таких, что $f_{2}(\lambda)-f_{1}(\lambda)=1 /\left(\lambda_{1} \lambda_{2}\right)$ в $V_{12}:=V_{1} \cap V_{2}$.

В слоях $\widetilde{M}_{\lambda}$ введем соотношение эквивалентности: $z_{j}^{\prime} \sim z_{j}$, если $z_{j}^{\prime}-z_{j}=$ $n+i m$ с некоторыми $n, m \in \mathbb{Z}$. Фактор $\widetilde{M}$ по этой (голоморфной) эквивалентности является голоморфным семейством торов $\pi: M \rightarrow B$, причем все торы $M_{\lambda}$ между собою конформно эквивалентны и $\widetilde{M}$ является универсальным накрывающим многообразия $M$. 
Покажем, что семейство $\tilde{\pi}: \widetilde{M} \rightarrow B$ не эквивалентно $B \times \mathbb{C} \rightarrow B$. В самом деле, предположим, что существует голоморфная на $\widetilde{M}$ функция $w$, однолистная в слоях. Тогда $w=a_{j} z_{j}+b_{j}$ в $U_{j}$ с коэффициентами, голоморфными в $V_{j}$, причем $a_{j}$ не имеют нулей. $\mathrm{B} U_{1} \cap U_{2}$ получаем равенство $a_{1} z_{1}+b_{1}=$ $a_{2}\left(z_{1}+1 /\left(\lambda_{1} \lambda_{2}\right)\right)+b_{2}$, откуда $a_{1}=a_{2}$ и, следовательно, $a:=a_{j}$ в $V_{j}$ есть целая функция без нулей в $\mathbb{C}^{2}$ (начало координат - устранимая особенность). Таким образом, $b_{1}-b_{2}=a /\left(\lambda_{1} \lambda_{2}\right)$ в $V_{12}$. Из равенства $\lambda_{1} \lambda_{2} b_{1}=a+\lambda_{1} \lambda_{2} b_{2}$ получаем, что функция $\lambda_{1} b_{1}$ голоморфно продолжается в $V_{2}$ (как и правая часть равенства) и, значит, $\lambda_{1} b_{1}$ продолжается до целой функции $h_{1}$. Аналогично, $\lambda_{2} b_{2}$ продолжается до целой функции $h_{2}$. А тогда $\lambda_{2} h_{1}-\lambda_{1} h_{2}=a$ всюду в $\mathbb{C}^{2}$, в частности, в начале координат. Это противоречие (с тем, что а не имеет нулей) показывает, что $\widetilde{M}$ не эквивалентно $B \times \mathbb{C}$ и, следовательно, семейство торов $\pi: M \rightarrow B$ не униформизуемо.

Вместе с тем, проекция $\tilde{\pi}$ допускает глобальное $\mathbb{R}$-аналитическое сечение: $f_{1}(\lambda)=\bar{\lambda}_{2} /\left(\lambda_{1}|\lambda|^{2}\right)$ в $V_{1}, f_{2}(\lambda)=\bar{\lambda}_{1} /\left(\lambda_{2}|\lambda|^{2}\right)$ в $V_{2}$. Его проекция в $M$ дает $\mathbb{R}$-аналитическое сечение проекции $\pi$.

Таким образом, $\pi: M \rightarrow B-$ неуниформизуемое голоморфное семейство торов, конформно эквивалентных между собою, голоморфно тривиальное над $V_{1}$ и $V_{2}$, причем проекция $\pi$ допускает глобальное $\mathbb{R}$-аналитическое сечение.

И все дело здесь в том, что база $B$ обладает одним "голоморфным" дефектом: первая проблема Кузена на ней для покрытия $B=V_{1} \cup V_{2}$ и данных $1 /\left(\lambda_{1} \lambda_{2}\right)$ на $V_{12}$ неразрешима (в классе голоморфных функций, конечно).

\section{2. Голоморфные движения и униформизация}

2.1. Классические голоморфные движения. Традиционное определение голоморфных движений (см. [45], [4], [5]) следующее.

Пусть $E$ - подмножество сферы Римана $\widehat{\mathbb{C}}$ и $\mathbb{D} \subset \mathbb{C}$ - единичный круг.

Отображение $\phi: \mathbb{D} \times E \rightarrow \widehat{\mathbb{C}}$ называется голоморфным движением (множества $E$ над $(\mathbb{D}, 0)$ или просто над $\mathbb{D})$, если

(i) $\phi(0, z)=z$ для всех $z \in E$,

(ii) для всякого $\lambda \in \mathbb{D}$ отображение $\phi(\lambda, \cdot): E \rightarrow \widehat{\mathbb{C}}$ есть вложение,

(iii) для всякого $z \in E$ отображение $\phi(\cdot, z): \mathbb{D} \rightarrow \widehat{\mathbb{C}}$ голоморфно.

Геометрически нагляднее отображение $\Phi:(\lambda, z) \mapsto(\lambda, w=\phi(\lambda, z))$. Его образ $E_{\phi}$, который мы будем называть пространством движения $\phi$, представляет собою объединение попарно непересекающихся (условие (ii)) мероморфных графиков $w=\phi(\lambda, z)$ (условие $(\mathrm{iii}))$ над $\mathbb{D}$ с отмеченными точками $w=z \in E$ в пересечениях с $0 \times \widehat{\mathbb{C}}$ (условие $(\mathrm{i}))$; по-другому: точки множества $0 \times E$, не сталкиваясь, двигаются в $\mathbb{D} \times \widehat{\mathbb{C}}$ голоморфно по "времени" $\lambda \in \mathbb{D}$ (отсюда и название).

Никакой непрерывности $\phi$ по совокупности переменных в определении a priori не предполагается, однако это следует из (i)-(iii), см. ниже.

Вместо круга $\mathbb{D}$ в качестве пространства параметров (базы) можно брать произвольное линейно связное комплексное банахово многообразие $B$ с выделенной (базовой) точкой $\lambda_{0}$ (заменяющей $\lambda=0$ в (i)). 
Голоморфное движение $\phi$ множества $E \subset \widehat{\mathbb{C}}$ над $\left(B, \lambda_{0}\right)$ называется нормированнъци, если $0,1, \infty \in E$ и $\phi(\lambda, 0) \equiv 0, \phi(\lambda, 1) \equiv 1, \phi(\lambda, \infty) \equiv \infty$ (точки $0,1, \infty \in \widehat{\mathbb{C}}$ неподвижны при изменении $\lambda \in B$ ). Движение $\phi: B \times E \rightarrow \mathbb{C}$ подмножества $E \subset \mathbb{C}$ называем нормированным, если точки $0,1 \in E$ остаются неподвижными при изменении $\lambda \in B$. Это важное свойство, поскольку для нормированного движения $\phi$ точки $z \in E \backslash\{0,1, \infty\}$ голоморфно по $\lambda$ меняются в гиперболической области $\mathbb{C} \backslash\{0,1\}$. Оно достигается стандартным дробно-линейным преобразованием $\widehat{\mathbb{C}}_{w}$ : если $\psi_{0}, \psi_{1}, \psi_{\infty}$ - голоморфные отображения $B \rightarrow \widehat{\mathbb{C}}$, графики которых попарно не пересекаются, то однозначно определено биголоморфное послойное преобразование многообразия $B \times \widehat{\mathbb{C}}$, переводящее графики функций $\psi_{j}$ соответственно в графики постоянных функций $w^{\prime}=0,1, \infty$. Таким образом, всякое голоморфное движение $B \times E \rightarrow \widehat{\mathbb{C}}$, $\# E \geqslant 3$, над произвольным комплексным банаховым многообразием $B$ допускает нормировку, и изучение голоморфных движений подмножеств $\widehat{\mathbb{C}}$ над $B$ при $\# E \geqslant 3$ сводится к изучению голоморфных движений подмножеств комплексной плоскости $\mathbb{C}$, т. е. систем соответствующих голоморфных (а не мероморфных) функций.

Большую роль в теории голоморфных движений играет гиперболическая геометрия, поэтому для удобства читателей приведем необходимый минимум о метрике Кобаяши (подробнее см., например, [38], [43], [51]).

Пусть $B$ - произвольное линейно связное комплексное банахово многообразие. Очевидно, что любых точек $\lambda^{\prime}, \lambda$, лежащих в одном координатном шаре многообразия $B$, существует голоморфный диск $h: \mathbb{D} \rightarrow B$ такой, что $h(0)=\lambda^{\prime}$, $h(\zeta)=\lambda$ для некоторой $\zeta \in \mathbb{D}$. Так как $B$ линейно связное и любой путь $[0,1] \rightarrow B$ покрывается конечным числом координатных шаров, то для любых $\lambda^{\prime}, \lambda \in B$ существует конечная цепочка голоморфных дисков $h_{j}: \mathbb{D} \rightarrow B$ и точек $\zeta_{j} \in \mathbb{D}$ таких, что $h_{1}(0)=\lambda^{\prime}, h_{j+1}(0)=h_{j}\left(\zeta_{j}\right), j=1, \ldots, N-1$, и $h_{N}\left(\zeta_{N}\right)=\lambda$. Величина $\kappa_{B}\left(\lambda^{\prime}, \lambda\right)=\inf \sum_{1}^{N} \log \frac{1+\left|\zeta_{j}\right|}{1-\left|\zeta_{j}\right|}$, где инфимум берется по всем описанным цепочкам голоморфных дисков ( $N$ может быть любым), называется расстоянием Кобаяии между точками $\lambda^{\prime}, \lambda$ на многообразии $B$. Из определения легко следуют симметричность и неравенство треугольника, но невырожденности в общем случае нет, расстояния между некоторыми различными точками могут равняться нулю.

Топология на $B$, определяемая метрикой (расстоянием) $\kappa_{B}$, в общем слабее топологии банахова многообразия $B$, шары $B_{R}(\lambda)=\left\{\lambda^{\prime}: \kappa_{B}\left(\lambda, \lambda^{\prime}\right)<R\right\}$ являются открытыми подмножествами $B$. Для выделенной (базовой) точки $\lambda_{0}$ шар $B_{R}\left(\lambda_{0}\right)$ будем обозначать просто $B_{R}$.

Многообразия $B$, для которых $\kappa_{B}$ есть настоящее расстояние (положительное вне диагонали в $B \times B$ ), называются гиперболическими. Например, если $B=\mathbb{B}$ - единичный шар в банаховом пространстве $(X,\|\cdot\|)$, то $\kappa_{\mathbb{B}}(\lambda, 0)=$ $\log \frac{1+\|\lambda\|}{1-\|\lambda\|}$, многообразие $\mathbb{B}$ гиперболическое и $\mathbb{B}_{R}=\mathbb{B}_{R}(0)$ есть шар $\|\lambda\|<$ $\operatorname{th}(R / 2)$. Другой пример - все римановы поверхности, у которых универсальной накрывающей является круг $\mathbb{D}$. 
Метрика Кобаяши естественно поднимается на произвольное накрывающее многообразие (поскольку любой диск поднимается), и это поднятие локально совпадает с метрикой Кобаяши накрывающего многообразия. Таким образом, для любой гиперболической римановой поверхности $S$ поднятие метрики $\kappa_{S}$ на $\mathbb{D}$ относительно универсального накрытия равно $\kappa_{\mathbb{D}}$, а для круга метрика $\kappa_{\mathbb{D}}$ совпадает с метрикой Пуанкаре $\rho_{\mathbb{D}}$, инвариантной относительно дробно-линейных автоморфизмов $\mathbb{D}$, с инфинитезимальным представлением $\rho_{\mathbb{D}}=\frac{2|d \zeta|}{1-|\zeta|^{2}}($ кривизны $\equiv-1)$.

Большую роль в изучении голоморфных движений играет гиперболическая область $\mathbb{C} \backslash\{0,1\}$ с полной невырожденной гиперболической метрикой Пуанкаре $\rho_{0,1}$. При универсальном отображении $\mathbb{D} \rightarrow \mathbb{C} \backslash\{0,1\}$ эта $\rho_{0,1}$ поднимается до метрики Пуанкаре $\rho_{\mathbb{D}}$. Так как поднятие в $\mathbb{D}$ метрики Кобаяши на $\mathbb{C} \backslash\{0,1\}$ такое же, то $\rho_{0,1}$ совпадает с метрикой Кобаяши этой области.

Важнейшим свойством метрики Кобаяши является ее очевидное невозрастание при голоморфных отображениях: если $f: B \rightarrow X$ - такое отображение, Tо

$$
\kappa_{X}\left(f(\lambda), f\left(\lambda^{\prime}\right)\right) \leqslant \kappa_{B}\left(\lambda, \lambda^{\prime}\right)
$$

(это один из абстрактных вариантов леммы Швариа).

Лемма Шварца (1) показывает, что

относительно расстояний Кобаяши любое голоморфное отображение удовлетворяет условию Липшища с константой 1.

В частности, для произвольного нормированного голоморфного движения $\phi: B \times E \rightarrow \widehat{\mathbb{C}}$ и любой точки $z \in \mathbb{C} \backslash\{0,1\}$ получаем оценку

$$
\rho_{0,1}\left(\phi\left(\lambda^{\prime}, z\right), \phi(\lambda, z)\right) \leqslant \kappa_{B}\left(\lambda^{\prime}, \lambda\right),
$$

которая означает, что гиперболическая скорость движения точки $z$ во всех точках графика $w=\phi(\lambda, z)$ не превосходит единицы.

Более деликатное свойство голоморфных движений в "вертикальном" направлении связано с оценкой искажения двойных отношений четверок точек. Для упорядоченного множества $\Sigma=\left\{z_{1}, \ldots, z_{4}\right\} \subset \widehat{\mathbb{C}}$ (точки $z_{j}$ различные) обозначим через $\operatorname{cr}(\Sigma)$ двойное отношение

$$
\operatorname{cr}(\Sigma)=\frac{z_{1}-z_{3}}{z_{1}-z_{4}}: \frac{z_{2}-z_{3}}{z_{2}-z_{4}}
$$

естественно сокращаемое $\left(\frac{\infty}{\infty}=1\right)$, если одна из точек равна $\infty$, и инвариантное относительно дробно-линейных преобразований сферы Римана. Тогда $\operatorname{cr}(\Sigma) \neq 0,1, \infty$, т. е. точка $\operatorname{cr}(\Sigma)$ лежит в гиперболической области $\mathbb{C} \backslash\{0,1\}$.

Лемма 2.1. Пусть $B$ - комплексное банахово многообразие, $\phi: B \times E \rightarrow \widehat{\mathbb{C}}-$ голоморфное движение над $\left(B, \lambda_{0}\right)$ множества $E \subset \widehat{\mathbb{C}} u \Sigma=\left\{z_{1}, \ldots, z_{4}\right\}$ состоuт из различных точек $E$. Тогда для всякого $\lambda \in B$ справедлива следующая ощенка для двойных отношений:

$$
\rho_{0,1}(\operatorname{cr}(\phi(\lambda, \Sigma)), \operatorname{cr}(\Sigma)) \leqslant \kappa_{B}\left(\lambda, \lambda_{0}\right),
$$

где $\phi(\lambda, \Sigma)=\left\{\phi\left(\lambda, z_{1}\right), \ldots, \phi\left(\lambda, z_{4}\right)\right\}$. 
ДоказАтельство. Отображение $\lambda \mapsto \operatorname{cr}(\phi(\lambda, \Sigma))$ из $B$ в $\mathbb{C} \backslash\{0,1\}$ голоморфно и равно $\operatorname{cr}(\Sigma)$ при $\lambda=\lambda_{0}$, а метрика Кобаяши при таком отображении не возрастает (неравенство (1)).

СлеДСТвИЕ 2.1. Пусть $f$ - голоморфная функиия на $B$ со значениями в $\mathbb{C} \backslash\{0,1\}$ и $R, R^{\prime}>0$ - константы, причем $\left|f\left(\lambda_{0}\right)\right| \leqslant R^{\prime}$. Тогда существует константа $M=M\left(R, R^{\prime}\right)$, зависящая только от $R, R^{\prime}$, такая, что $|f(\lambda)|<M$ для всех $\lambda \in B_{R}$.

ДокАзАТЕЛЬСтво. Применим неравенства (3) к голоморфному движению $0,1, f(\lambda)$ множества $0,1, f\left(\lambda_{0}\right)$ и воспользоваться тем, что расстояние до $\infty$ в метрике $\rho_{0,1}$ равно бесконечности.

Это свойство a priori совсем не очевидно, поскольку замкнутые шары Кобаяши не обязательно компактные даже в конечномерном случае.

Из леммы 2.1 и оценок снизу метрики Пуанкаре в $\mathbb{C} \backslash\{0,1\}$ следует, что голоморфные движения удовлетворяют условию Гёльдера по $z$ в сферической метрике. Точнее, справедливо следующее утверждение (доказательство см. в [15], [35]).

Лемма 2.2. Пусть $\phi: B \times E \rightarrow \mathbb{C}$ - нормированное голоморфное движение над комплексным банаховым многообразием $\left(B, \lambda_{0}\right)$. Тогда для любых $R, R^{\prime}>0$ существует константа $C=C\left(R, R^{\prime}\right)$ такая, что

$$
\left|\phi(\lambda, z)-\phi\left(\lambda, z^{\prime}\right)\right| \leqslant C\left|z-z^{\prime}\right|^{\alpha}, \quad \alpha=e^{-R},
$$

для всех $\lambda \in B_{R} u z, z^{\prime} \in E \cap R^{\prime} \mathbb{D}$.

Вместе со следствием 2.1 это дает равностепенную оценку модуля непрерывности для всех нормированных голоморфных движений над $\left(B, \lambda_{0}\right)$.

СлеДСтвиЕ 2.2. Для $\lambda, \lambda^{\prime} \in B_{R} u z, z^{\prime} \in E \cap R^{\prime} \mathbb{D}$

$$
\left|\phi(\lambda, z)-\phi\left(\lambda^{\prime}, z^{\prime}\right)\right| \leqslant C_{1} \kappa_{B}\left(\lambda, \lambda^{\prime}\right)+C_{2}\left|z-z^{\prime}\right|^{\alpha}
$$

с константами $C_{1}, C_{2}$, зависящими только от $R, R^{\prime}, u \alpha=e^{-R}$.

Доказательство. По следствию $2.1,\left|\phi\left(\lambda, z^{\prime}\right)-\phi\left(\lambda^{\prime}, z^{\prime}\right)\right| \leqslant 2 M$, где $M=$ $M\left(R, R^{\prime}\right)$. Для точек $w, w^{\prime} \in 2 M \mathbb{D}$ евклидово расстояние не меньше, чем $C(M) \rho_{0,1}\left(w, w^{\prime}\right)$ с некоторой константой $C(M)$, и, значит, согласно неравенству (2),

$$
\left|\phi\left(\lambda, z^{\prime}\right)-\phi\left(\lambda^{\prime}, z^{\prime}\right)\right| \leqslant C(M) \kappa_{B}\left(\lambda, \lambda^{\prime}\right) .
$$

СледСТвиЕ 2.3. Пусть $\left\{E_{j} \subset \mathbb{C}\right\}$ - возрастающая последовательность подмножеств, $E$ - замыкание $\bigcup E_{j} u \phi_{j}: B \times E_{j} \rightarrow \mathbb{C}$ - нормированные голоморфные движения над $\left(B, \lambda_{0}\right)$ такие, что $\phi_{j+1} \mid B \times E_{j}=\phi_{j}$ для всех $j$. Тогда существует единственное голоморфное движение $\phi: B \times E \rightarrow \mathbb{C}$ такое, что $\left.\phi\right|_{B \times E_{j}}=\phi_{j} \partial л я$ всех $j$.

ДоказАтельство. Пусть $\left\{z_{j} \in E_{j}\right\}$ - последовательность различных точек множества $E$, сходящаяся к некоторой $z_{0} \in E$. По лемме $2.2, \mid \phi_{j}\left(\lambda, z_{j}\right)-$ $\phi_{k}\left(\lambda, z_{k}\right)\left|\leqslant C_{1}\right| z_{j}-\left.z_{k}\right|^{\alpha}$ для $\lambda \in B_{R}$ и, значит, по критерию Коши, последовательность функций $\phi_{j}\left(\lambda, z_{j}\right): B \rightarrow \mathbb{C}$ сходится равномерно на каждом $B_{R}$ к некоторой функции $\psi(\lambda)$, голоморфной по теореме Вейерштрасса. 
Так как это верно для любой последовательности точек $z_{j} \in E_{j}$, сходящейся к $z_{0}$, то $\psi$ зависит только от $z_{0}$ и мы определяем $\phi\left(\lambda, z_{0}\right):=\psi(\lambda)$. Проделав это для каждой предельной точки множества $\bigcup E_{j}$, получаем отображение $\phi: B \times$ $E \rightarrow \widehat{\mathbb{C}}$ такое, что $\phi$ голоморфно по $\lambda$ и $\phi\left(\lambda_{0}, z\right)=z$ для всех $z \in E$.

Свойство (ii) голоморфных движений, а именно инъективность по $z$, достаточно проверить над произвольным голоморфным диском, т. е. можно считать, что $B_{R}=\mathbb{D}$ и $|\phi(\lambda, z)| \leqslant M$. Для $z, z^{\prime} \in E, z \neq z^{\prime}$, функция $u(\lambda)=$ $\log \left(2 M /\left|\phi(\lambda, z)-\phi\left(\lambda, z^{\prime}\right)\right|\right)$ является пределом положительных в $\mathbb{D}$ гармонических функций $\log \left(2 M /\left|\phi_{j}\left(\lambda, z_{j}\right)-\phi_{j}\left(\lambda, z_{j}^{\prime}\right)\right|\right)$ с $z_{j}, z_{j}^{\prime} \in E_{j}, z_{j} \rightarrow z, z_{j}^{\prime} \rightarrow z^{\prime}$, $\left|z_{j}-z_{j}^{\prime}\right| \geqslant\left|z-z^{\prime}\right| / 2>0$. Так как для них справедливы неравенства Харнака (см., например, [33]), то $u(\lambda)$ равномерно ограничена в $r \mathbb{D}, r<1$, в частности, $\phi(\lambda, z) \neq \phi\left(\lambda, z^{\prime}\right)$ для всех $\lambda \in r \mathbb{D}$ и, значит, для всех $\lambda \in \mathbb{D}$, ввиду произвольности $r$. Следствие доказано.

Это упрощает многие доказательства. Например, для произвольного $E$ в качестве $E_{j}$ можно брать его конечные подмножества, объединение которых всюду плотно в $E$.

Неравенство (3) означает, пожалуй, важнейшее свойство голоморфных движений $\phi: B \times E \rightarrow \widehat{\mathbb{C}}$, а именно, квазиконформность отображений $\phi(\lambda, \cdot): E \rightarrow$ $\widehat{\mathbb{C}}$ при каждом фиксированном $\lambda$.

Для произвольного множества $E \subset \widehat{\mathbb{C}}$ одно из возможных определений квазиконформности отображения $f: E \rightarrow \widehat{\mathbb{C}}$ (см. [59]) заключается как раз в том, что $\rho_{0,1}(\operatorname{cr}(f(\Sigma)), \operatorname{cr}(\Sigma))<C$ для любой упорядоченной четверки $\Sigma$ различных точек в $E$ с некоторой единой константой $C=C(E)<\infty$. Для открытых множеств $E$ из этого следует, как хорошо известно (см., например, [42]), квазиконформность $f$ в любых ее традиционных определениях.

Квазиконформные отображения открытых подмножеств $\mathbb{C}$ дифференцируемы почти всюду и их первые частные производные (определенные почти всюду) совпадают с соответствующими частными производными в смысле распределений (см. [42]). Поэтому почти всюду определена характеристика (коэффициент Бельтрами) $\mu_{f}=f_{\bar{z}} / f_{z}$ такого отображения $f$, где $f_{z}, f_{\bar{z}}-$ формальные производные $f$ по $z$ и $\bar{z}$. Так как квазиконформные отображения сохраняют ориентацию, то почти всюду $\left|\mu_{f}\right|<1$.

Берс и Ройден [12] показали, что для любого голоморфного движения $\phi$ открытого подмножества $\mathbb{C}$ характеристика (коэффициенты Бельтрами) квазиконформных отображений $z \mapsto \phi(\lambda, z)$ голоморфно зависит от $\lambda \in B$, точнее, справделива следующая лемма.

Лемма 2.3. Пусть $\phi: B \times \Omega \rightarrow \mathbb{C}$ - голоморфное движение открытого множества $\Omega \subset \mathbb{C}$ над почти комплексным банаховым многообразием $B$ с отмеченной точкой $\lambda_{0} u \mu(\lambda, \cdot)=\left(\phi_{\bar{z}} / \phi_{z}\right)(\lambda, \cdot)$. Тогда отображение $B \ni \lambda \mapsto$ $\mu(\lambda, \cdot) \in L^{\infty}(\Omega)$ голоморфно.

(Ограниченное отображение почти комплексного многообразия $B$ голоморфно, если голоморфны его сужения на произвольные голоморфные диски $f$ : $\mathbb{D} \rightarrow$ B.)

ДокАЗАТЕЛЬство. Так как пространство $L^{\infty}(\Omega)$ - сопряженное к $L^{1}(\Omega)$, то достаточно доказать, что ограниченная функция $\Psi(\lambda)=\int \mu(\lambda, z) \psi(z) d x d y$, 
$z=x+i y$, голоморфна для всякой $\psi \in L^{1}(\Omega)$ с компактным носителем в $\Omega$ (такие $\psi$ образуют тотальное множество непрерывных линейных функционалов на $\left.L^{\infty}(\Omega)\right)$.

По доказанному выше, отображение $\phi(\lambda, \cdot): \Omega \rightarrow \mathbb{C}$ квазиконформно, в частности, дифференцируемо почти всюду в $\Omega$ и, значит,

$$
\Psi(\lambda)=\int \psi(z) \frac{\phi_{x}+i \phi_{y}}{\phi_{x}-i \phi_{y}}(\lambda, z) d x d y=\int \psi(z) \lim _{t \rightarrow 0} \frac{1+i \sigma}{1-i \sigma}(\lambda, z, t) d x d y
$$

где

$$
\sigma(\lambda, z, t)=\frac{\phi(\lambda, z+i t)-\phi(\lambda, z)}{\phi(\lambda, z+t)-\phi(\lambda, z)} .
$$

Для фиксированных $z \in K=\operatorname{supp} \psi$ и $0<t<\operatorname{dist}(K, \partial \Omega) / 2$ функция $\sigma(\lambda, z, t)$ определена, голоморфна по $\lambda \in B$, ее значения лежат в $\mathbb{C} \backslash\{0,1\}$ и $\sigma\left(\lambda_{0}, z, t\right)=i$. По свойству невозрастания метрики Кобаяши, из этого следует, что

$$
\rho_{0,1}(\sigma(\lambda, z, t), i) \leqslant \kappa_{B}\left(\lambda, \lambda_{0}\right)<r
$$

на $B_{r}$ и, значит, $|\sigma(\lambda, z, t)-i|<1 / 2$, если $r>0$ достаточно мало́ (такое $r$ есть, единое для всех указанных $z, t)$. Для $\lambda \in B_{r}$ имеем равномерную оценку $\left|\frac{1+i \sigma}{1-i \sigma}(\lambda, z, t)\right|<\frac{1}{3}$. По теореме Лебега об ограниченной сходимости, последовательность равномерно ограниченных на $B_{r}$ голоморфных функций

$$
\Psi_{n}(\lambda)=\int_{K} \psi(z) \frac{1+i \sigma(\lambda, z, 1 / n)}{1-i \sigma(\lambda, z, 1 / n)} d x d y
$$

при $n \rightarrow \infty$ сходится поточечно в $B_{r}$ к $\Psi(\lambda)$ и, значит, $\Psi$ голоморфна в $B_{r}$. Таким образом, мы получаем, что отображение $\mu(\lambda, \cdot) \mid B_{r} \rightarrow L^{\infty}(\Omega)$ голоморфно.

Пусть теперь $\lambda_{1} \in B$ - произвольная точка, $\Omega_{1}:=\phi\left(\lambda_{1}, \Omega\right)$ и $\mu_{1}(z)$ - характеристика квазиконформного отображения $\phi\left(\lambda_{1}, \cdot\right): \Omega \rightarrow \Omega_{1}$. Тогда характеристика $\nu(\lambda, z)$ отображения $\phi(\lambda, \cdot) \circ \phi\left(\lambda_{1}, \cdot\right)^{-1}: \Omega_{1} \rightarrow \Omega_{\lambda}$ в точке $\phi\left(\lambda_{1}, z\right)$ по известной формуле для композиции квазиконформных отображений (см. $[1 ; \mathrm{IC}])$ равна

$$
\frac{\mu(\lambda, z)-\mu_{1}(z)}{1-\overline{\mu_{1}(z)} \mu(\lambda, z)} \frac{\phi_{z}\left(\lambda_{1}, z\right)}{\overline{\phi_{z}\left(\lambda_{1}, z\right)}} .
$$

Применяя доказанное выше к голоморфному движению $\phi(\lambda, \cdot) \circ \phi\left(\lambda_{1}, \cdot\right)^{-1}$ множества $\Omega_{1}$ над $\left(B, \lambda_{1}\right)$, получаем, что отображение $\nu(\lambda, \cdot): B_{r_{1}}\left(\lambda_{1}\right) \rightarrow L^{\infty}\left(\Omega_{1}\right)$ голоморфно, а из этого, очевидно, следует голоморфность $\mu(\lambda, \cdot)$ в $B_{r_{1}}\left(\lambda_{1}\right)$. Лемма доказана.

Напомним, что расстояние Каратеодори на комплексном банаховом многообразии $B$ определяется как

$$
c_{B}\left(\lambda, \lambda^{\prime}\right)=\sup \left\{\log \frac{1+|f(\lambda)|}{1-|f(\lambda)|}\right\}
$$

где супремум берется по всем голоморфным функциям $f: B \rightarrow \mathbb{D}$ таким, что $f\left(\lambda^{\prime}\right)=0$. В общем случае $c_{B} \leqslant \kappa_{B}$, хотя в банаховом шаре (в частности, в $\mathbb{D}$ ) эти метрики совпадают.

Так как $\mu\left(\lambda_{0}, z\right) \equiv 0$ в $\Omega$ и $|\mu|<1$, то из леммы Шварца получаем следующее утверждение. 
СлЕДСтвиЕ 2.4. Для всех $z \in \Omega$

$$
\|\mu(\lambda, \cdot)\|_{\infty} \leqslant \operatorname{th} \frac{c_{B}\left(\lambda, \lambda_{0}\right)}{2} .
$$

$B$ частности, если $B$ - единичный шар в комплексном банаховом пространстве с нормой $\|\cdot\|$ u $\lambda_{0}=0$, mо $\|\mu(\lambda, \cdot)\|_{\infty} \leqslant\|\lambda\|$.

Для коэффициента квазиконформности $K_{\phi(\lambda, \cdot)}=\frac{1+\|\mu(\lambda, \cdot)\|_{\infty}}{1-\|\mu(\lambda, \cdot)\|_{\infty}}$ имеет место соответствующая оценка, $K_{\phi(\lambda, \cdot)} \leqslant e^{c_{B}\left(\lambda, \lambda_{0}\right)}$.

Между прочим, $\mu \equiv 0$, если на $B$ нет непостоянных ограниченных голоморфных функций (такие многообразия называются лиувиллевыми). А тогда $\phi_{\bar{z}}(\lambda, \cdot)=0$ почти всюду и из леммы Вейля следует, что $\phi$ голоморфно не только по $\lambda$, но и по $z$, т. е. $\phi: B \times \Omega \rightarrow \mathbb{C}$ - глобально голоморфное отображение.

Доказанные в этом пункте свойства голоморфных движений составляют содержание так называемой $\lambda$-леммы (этот термин стал уже общепринятым), доказанной в главном в [45] для базового случая $B=\mathbb{D}$.

Лемма 2.4 ( $\lambda$-лемма). Пусть $\phi: B \times E \rightarrow \mathbb{C}$ - голоморфное движение множества $E \subset \mathbb{C}$ над единичнъм шаром $(B, 0)$ комплексного банахова пространства. Тогда

1) ф непрерывно по совокупности переменных $(\lambda, z)$,

2) ф единственным образом продолжается до голоморфного движения замыкания множества $E$,

3) если $E$ открыто, то отображения $\phi(\lambda, \cdot): E \rightarrow \mathbb{C}$ квазиконформны при каждом фиксированном $\lambda$, причем отображение характеристики движения $\lambda \mapsto \mu(\lambda, \cdot)$ голоморфно $и\|\mu(\lambda, \cdot)\|_{\infty} \leqslant\|\lambda\|$.

2.2. Горизонтально голоморфные тривиализации. Пусть $\pi: M \rightarrow B-$ голоморфное семейство римановых поверхностей. Фиксируем точку $\lambda_{0} \in B$ и множество $E \subset M_{0}:=M_{\lambda_{0}}$ (слой $M_{0}$ "базовый"). Отображение $\psi: B \times E \rightarrow M$ называется голоморфным движением (множества $E$ над $\left(B, \lambda_{0}\right)$ ), если

(i) $\psi\left(\lambda_{0}, z\right) \equiv z$ для всех $z \in E$,

(ii) для всякой $\lambda \in B$ множество $\psi(\lambda, E)$ лежит в $M_{\lambda}$ и отображение $\psi(\lambda, \cdot)$ : $E \rightarrow M_{\lambda}$ является вложением,

(iii) для всякого $z \in E$ отображение $\psi(\cdot, z): B \rightarrow M$ голоморфно.

$(E$ и $\phi$ из определения классического движения, с $M=B \times \widehat{\mathbb{C}}$, равны соответственно $\pi^{\prime}(E)$ и $\pi^{\prime} \circ \psi$, где $\pi^{\prime}-$ проекция на $\widehat{\mathbb{C}}$.)

Свойство (ii) в определении означает, в частности, что для каждого $z \in E$ отображение $\psi(\cdot, z)$ является сечением проекции $\pi$, т. е. $\pi \circ \psi(\lambda, z) \equiv \lambda$, a (iii) говорит, что это сечение голоморфно. Таким образом, геометрически, голоморфное движение в расслоенном пространстве $\pi: M \rightarrow B$ есть совокупность попарно непересекающихся образов $\Gamma_{z}, z \in E$, голоморфных сечений проекции $\pi$. Их объединение $E_{\phi}=\bigcup_{z \in E} \Gamma_{z}$, как и в декартовой ситуации, называем пространством движения $\phi$. Если $E_{\phi}=M$, то мы говорим, что движение $\phi$ заполняет $M$ (или $M$ заполняется движением $\phi$ ), а отображение $\phi: B \times M_{0} \rightarrow M$ называем тогда горизонтально голоморфной тривиализацией семейства $\pi: M \rightarrow B$. 
Возникает естественный вопрос о справедливости утверждений, доказанных в предыдущем разделе, для случая голоморфных движений в таких расслоенных пространствах.

Непрерывность по совокупности переменных - локальное свойство, поэтому она есть и в общем случае.

Вопрос о продолжении на замыкание более деликатный. Простой пример $M=\left\{(\lambda, z) \in \mathbb{C}^{2}:|z|<1+|\lambda|\right\}, B=\mathbb{D}, \lambda_{0}=1 / 2$ и $E=\mathbb{D}$ показывает, что необходимо некоторое условие типа псевдовыпуклости $M$ в "бесконечности вдоль слоев", но далее это свойство движений не используется и мы лишь констатируем наличие некоторой проблемы.

Композиции квазиконформных отображений квазиконформны, поэтому отображения $E \rightarrow \phi(\lambda, E)$ локально квазиконформны и локально удовлетворяют соответствующим условиям Гёльдера. С глобальной квазиконформностью ситуация посложнее, и мы исследуем ее подробнее, поскольку именно квазиконформность является главным свойством, позволяющим применять голоморфные движения к униформизации семейств.

Пусть $\phi: B \times M_{0} \rightarrow M$ - горизонтально голоморфная тривиализация голоморфного семейства $\pi: M \rightarrow B, M_{0}:=M_{\lambda_{0}}$ и $f_{0}: D \rightarrow M_{0}$ - голоморфное накрытие поверхности $M_{0}$ областью $D \subset \mathbb{C}$, обеспечивающее локальные голоморфные координаты на $M_{0}$ (далее нам понадобятся в основном общие универсальные накрытия $f_{0}$, но односвязность $D$ пока не нужна). Тогда $\tilde{\phi}: B \times$ $D \rightarrow M$, где $\tilde{\phi}(\lambda, z):=\phi\left(\lambda, f_{0}(z)\right)$ - топологическое накрытие, голоморфное по $\lambda$. Будучи локально квазиконформным по $z$, оно почти всюду дифференцируемо по $z$ и определенные почти всюду в $D$ первые частные производные совпадают с частными производными в смысле распределений.

Если $w$ - голоморфная функция в окрестности точки $\tilde{\phi}(\lambda, z)$ на $M$ такая, что $d w \mid M_{\lambda} \neq 0$ в этой точке, и $W=w \circ \tilde{\phi}$, то $d W=a(z)(d z+\mu(\lambda, z) d \bar{z})$ с функцией $\mu$, a priori зависящей от $w$. Однако, если $w_{1}-$ другая такая функция, то $d w_{1}\left|M_{\lambda}=c(\lambda, w) d w\right| M_{\lambda}$ и потому функция $\mu(\lambda, \cdot)$ при каждом $\lambda \in B$ определена почти всюду в $D$ независимо от выбора локальных координат в $M$. Как и выше, мы называем ее характеристикой голоморфного движения (тривиализации) $\phi$. При $\lambda=\lambda_{0}$ функция $W\left(\lambda_{0}, z\right)$, как выше, равна $w \circ f_{0}(z)$, поэтому она голоморфна по $z$ и, значит, $\mu\left(\lambda_{0}, \cdot\right)=0$.

Функция $\mu$, конечно, зависит и от $\lambda_{0}$, и от $D$, и от накрытия $f_{0}: D \rightarrow M_{0}$, и мы будем их указывать там, где это понадобится. Отметим, однако, что $|\mu|$ от выбора локальных "вертикальных" координат $z$ не зависит.

Лемма 2.5. Пусть $\pi: M \rightarrow B$ - голоморфное семейство римановых поверхностей над комплексным банаховым многообразием $\left(B, \lambda_{0}\right)$ с базовым слоем $M_{0}=\pi^{-1}\left(\lambda_{0}\right), f_{0}: D \rightarrow M_{0}$ - фиксированное голоморфное накрытие областью $D \subset \mathbb{C}, \phi: B \times M_{0} \rightarrow M-$ горизонтально голоморфная тривиализация и $\mu$-соответствующая им характеристика. Тогда отображение $B \ni \lambda \mapsto \mu(\lambda, \cdot) \in L^{\infty}(D)$ голоморфно $и\|\mu(\lambda, \cdot)\|_{\infty} \leqslant \operatorname{th}\left(c_{B}\left(\lambda, \lambda_{0}\right) / 2\right)$.

Отметим сразу, что в единичном шаре $\mathbb{B}$ любого комплексного банахова пространства метрика Каратеодори $c_{\mathbb{B}}$ совпадает с метрикой Кобаяши $\kappa_{\mathbb{B}}$ и равна 
$\log \frac{1+\|\lambda\|}{1-\|\lambda\|}=2 \operatorname{arcth}\|\lambda\|$. Таким образом, для $B=\mathbb{B}$ получается классическое неравенство Шварца: $\|\mu(\lambda, \cdot)\|_{\infty} \leqslant\|\lambda\|$.

ДокАЗАТЕЛЬСтво. Удобно считать, что $\mu(\lambda, \cdot)$ продолжена нулем на $\mathbb{C} \backslash D$ и, таким образом, $\mu(\lambda, \cdot) \in L^{\infty}(\mathbb{C})$. Ограниченное отображение $\lambda \mapsto \mu(\lambda, \cdot)$ голоморфно тогда и только тогда, когда голоморфно его сужение на произвольный вложенный голоморфный диск $h: \mathbb{D} \rightarrow B$, т. е. когда голоморфно отображение $\zeta \mapsto \mu(h(\zeta), \cdot)$.

Шаг 1. Покажем сначала, что это верно для любого такого диска $h$ с центром $h(0)=\lambda_{0}$.

Зафиксируем $0<r<1$ и положим $\tilde{\phi}(\zeta, z)=\phi\left(h(\zeta), f_{0}(z)\right)$. Для любого $z_{1} \in$ $D$ голоморфный диск $S_{1}=\left\{\tilde{\phi}\left(\zeta, z_{1}\right): \zeta \in \mathbb{D}\right\}$ является многообразием Штейна. По теореме Сью [55], существует штейнова окрестность $V_{1} \supset S_{1}$ в $\pi^{-1}(h(\mathbb{D}))$, стягиваемая на $S_{1}$. Поэтому на $V_{1}$ есть голоморфная функция, равная $\zeta$ на $S_{1}$, и голоморфная функция $w$ такая, что $d w \wedge d \zeta \neq 0$ во всех точках $S_{1}$. Поэтому существуют окрестности $U_{1} \ni z_{1}$ в $D$ и $V_{1}^{\prime} \supset S_{1} \cap\{\zeta \in r \mathbb{D}\}$ в $\pi^{-1}(h(r \mathbb{D}))$ такие, что отображение $V_{1}^{\prime} \ni p \mapsto(\zeta(p), w(p)) \in r \mathbb{D} \times U_{1}$ биголоморфно.

Положим $W(\zeta, z):=w \circ \tilde{\phi}(\zeta, z)$; тогда $\left(W_{\bar{z}} / W_{z}\right)(\zeta, \cdot)=\mu(h(\zeta), \cdot)$ по определению характеристики. Так как $W(0, z) \equiv z$, то $W: r \mathbb{D} \times U_{1} \rightarrow \mathbb{C}$ - обычное голоморфное движение. По теореме Берса-Ройдена (лемма 2.3) отображение $\zeta \mapsto\left(W_{\bar{z}} / W_{z}\right)(\zeta, \cdot) \in L^{\infty}\left(U_{1}\right)$ голоморфно. Так как $\left(W_{\bar{z}} / W_{z}\right)(\zeta, \cdot)=\mu(h(\zeta), \cdot)$, то отображение $\zeta \mapsto \mu\left(h(\zeta), \chi_{1} \cdot\right) \in L^{\infty}(\mathbb{C})$, где $\chi_{1}$ - характеристическая функция $U_{1}$, голоморфно.

Уменьшая $U_{1}$, можем считать, что это есть диадический квадрат. Так как точка $z_{1} \in D$ в приведенном рассуждении произвольная, то существует покрытие $D$ диадическими квадратами $U_{j}$ с попарно непересекающимися внутренностями такое, что каждое отображение $\zeta \mapsto \mu\left(h(\zeta), \chi_{j} \cdot\right)$, где $\chi_{j}$ - характеристическая функция $U_{j}$, голоморфно.

Остается заметить, что $L^{\infty}(\mathbb{C})=\left(L^{1}(\mathbb{C})\right)^{*}$ и, следовательно, отображение $\zeta \longmapsto \mu(h(\zeta), \cdot)$ голоморфно тогда и только тогда, когда $\int_{D} \mu(h(\zeta), \cdot) \psi d S-$ голоморфная скалярная функция для всякой $\psi \in L^{1}(\mathbb{C})$. Но $\mu=\sum \chi_{j} \mu$ и ряд сходится к $\mu$ слабо- ${ }^{*}$, т. е. $\int \mu \psi d S=\sum \int \chi_{j} \mu \psi d S$. По доказанному выше, все члены ряда голоморфны по $\zeta \in r \mathbb{D}$ и, значит, функция $\int \mu(\lambda, z) \psi(z) d S_{z}$ голоморфна в $\mathbb{D}$ по теореме Вейерштрасса, ввиду произвольности $r$. По лемме Шварца, она по модулю не превосходит $|\zeta|\|\psi\|_{1}$ и потому $\|\mu(h(\zeta), \cdot)\|_{\infty} \leqslant|\zeta|$.

В качестве следствия получаем, что для любого координатного шара $\mathbb{B} \ni \lambda_{0}$ в $B$ все отображения $\phi(\lambda, \cdot): M_{0} \rightarrow M_{\lambda}, \lambda \in \mathbb{B}$, квазиконформны.

Шаг 2. Теперь заменим базовую точку $\lambda_{0}$ на $\lambda_{1} \in \mathbb{B}$ и тривиализацию $\phi$ на тривиализацию $\phi_{1}: B \times M_{1} \rightarrow M, M_{1}:=M_{\lambda_{1}}$, определяемую по формуле 
$\phi_{1}(\lambda, \cdot)=\phi(\lambda, \cdot) \circ \phi\left(\lambda_{1}, \cdot\right)^{-1}$. Область $D_{1} \subset \mathbb{C}_{\zeta}$, накрывающую $M_{1}$ и необходимую для определения характеристики, зададим как $g(D)$, где $g$ - гомеоморфное в $\mathbb{C}$ решение уравнения Бельтрами $g_{\bar{z}}=\mu\left(\lambda_{1}, \cdot\right) g_{z}$ с неподвижными точками 0,1 , а накрытие $f_{1}: D_{1} \rightarrow M_{1}$ определим как композицию $f_{1}=\tilde{\phi}\left(\lambda_{1}, \cdot\right) \circ g^{-1}$, где $\tilde{\phi}(\lambda, z):=\phi\left(\lambda, f_{0}(z)\right), z \in D$.

Если $w$ - голоморфная координата в некоторой области на $M_{1}$, однолистно накрываемой областью $U \subset D_{1}$, то $U \ni z \mapsto w \circ f_{1}(z)-$ квазиконформное отображение плоских областей. По определению, $\mu\left(\lambda_{1}, \cdot\right)=W_{\bar{z}} / W_{z}$, где $W=$ $w \circ \tilde{\phi}\left(\lambda_{1}, \cdot\right)$. Так как и $g_{\bar{z}} / g_{z}=\mu\left(\lambda_{1}, \cdot\right)$, то $\left(W \circ g^{-1}\right)_{\bar{\zeta}} /\left(W \circ g^{-1}\right)_{\zeta}=0$ почти всюду в $U$ и, значит (по лемме Вейля для квазиконформных отображений), функция $W \circ g^{-1}$ голоморфна в $U$. Ввиду произвольности $U$ это означает, что $f_{1}$ - голоморфное накрытие.

Пусть теперь $w$ - голоморфная функция в окрестности точки $q \in M_{1}$ на $M$ такая, что $d w \mid M_{1} \neq 0$ в этой точке, $z_{1} \in D_{1}$ такова, что $f_{1}\left(z_{1}\right)=q, \tilde{\phi}_{1}(\lambda, z):=$ $\phi_{1}\left(\lambda, f_{1}(z)\right), z \in D_{1}$, и $W^{1}:=w \circ \tilde{\phi}_{1}$ (в окрестности $\left.\left(\lambda_{1}, z_{1}\right)\right)$. Тогда характеристика $\mu_{1}$ тривиализации $\phi_{1}$, соответствующая накрытию $f_{1}$, равна $W_{\bar{z}}^{1} / W_{z}^{1}$. Распишем $W^{1}$ при фиксированном $\lambda$ :

$$
\begin{aligned}
W^{1} & =w \circ \phi_{1}(\lambda, \cdot) \circ f_{1}=w \circ \phi(\lambda, \cdot) \circ \phi\left(\lambda_{1}, \cdot\right)^{-1} \circ f_{1} \\
& =w \circ \phi(\lambda, \cdot) \circ f_{0} \circ g^{-1}=(w \circ \tilde{\phi}(\lambda, \cdot)) \circ g^{-1}=W \circ g^{-1},
\end{aligned}
$$

где $W=w \circ \tilde{\phi}$. Так как $W_{\bar{z}} / W_{z}=\mu$, а $g_{\bar{z}} / g_{z}=\mu\left(\lambda_{1}, \cdot\right)$ не зависит от $\lambda$, то по формуле коэффициента Бельтрами для композиции (см. [1; IC])

$$
\mu_{1}(\lambda, \cdot) \circ g=\frac{g_{z}}{\overline{g_{z}}} \frac{\mu(\lambda, \cdot)-\mu\left(\lambda_{1}, \cdot\right)}{1-\overline{\mu\left(\lambda_{1}, \cdot\right)} \mu(\lambda, \cdot)} .
$$

Согласно шагу $1, \mu_{1}$ (как отображение в $L^{\infty}(\mathbb{C})$ ) голоморфна на каждом голоморфном диске $h: \mathbb{D} \rightarrow B$ таком, что $h(0)=\lambda_{1}$. Из формулы для композиции следует, что $\mu$ тоже голоморфна на таких дисках и, значит, $\mu$ голоморфна в $\mathbb{B}$. Более того, формула показывает, что если $\mu$ голоморфна по $\lambda$ над какой-нибудь областью в $B\left(\right.$ как элемент $\left.L^{\infty}(\mathbb{C})\right)$, то там же голоморфна и $\mu_{1}$, и наоборот.

Шаг 3. Так как $B$ линейно связное, то для каждой точки $\lambda^{\prime} \in B$ существует конечная цепочка координатных шаров $\mathbb{B}_{j}, j=0, \ldots, N$, и точек $\lambda_{j}, \lambda_{j+1} \in \mathbb{B}_{j}$ таких, что $\lambda_{N+1}=\lambda^{\prime}$; следовательно, $\mu(\lambda, \cdot) \in L^{\infty}(\mathbb{C})$ голоморфна по $\lambda$ всюду B $B$.

Это свойство эквивалентно тому, что $\psi_{\mu}(\lambda):=\int \mu(\lambda, \cdot) \psi d S-$ скалярная голоморфная функция для любой $\psi \in L^{1}(\mathbb{C}),\|\psi\|_{1} \leqslant 1$, причем эта функция по модулю не превосходит 1. Так как расстояние Каратеодори не увеличивается при голоморфных отображениях, то $\log \frac{1+\left|\psi_{\mu}(\lambda)\right|}{1-\left|\psi_{\mu}(\lambda)\right|} \leqslant c_{B}\left(\lambda, \lambda_{0}\right)$, откуда $\|\mu(\lambda, \cdot)\|_{\infty} \leqslant \operatorname{th}\left(c_{B}\left(\lambda, \lambda_{0}\right) / 2\right)$. Лемма доказана.

Лемма 2.5 в основном (для $D=\mathbb{D}$ ) доказана в приложении к работе Эрла и Фоулера [20].

Имея такую замечательную функцию $\mu$ на $B \times D$, мы можем построить обычное голоморфное движение в $B \times \mathbb{C}$ с этой характеристикой, что, оказывается, приводит к одновременной униформизации семейства $\pi: M \rightarrow B$. 
Лемма 2.6. Пусть $\pi: M \rightarrow B$ - голоморфное семейство римановых поверхностей с базовым слоем $M_{0}$ над комплексным банаховым многообразием $\left(B, \lambda_{0}\right), \phi: B \times M_{0} \rightarrow M-$ горизонтально голоморфная тривиализация u $f_{0}: D \rightarrow M_{0}$ - голоморфное накрытие областью $D \subset \mathbb{C}$. Тогда существуют область $\Omega \subset B \times \mathbb{C}$ с $\Omega_{\lambda_{0}}=D$ и послойно голоморфное накрытие $f: \Omega \rightarrow M$ такое, что $f \mid D=f_{0}$. При этом однозначно определено нормированное голоморфное движение $\psi: B \times \mathbb{C} \rightarrow \mathbb{C}$, заполняющее $B \times \mathbb{C}$, голоморфное в $B \times(\mathbb{C} \backslash \bar{D})$ по совокупности переменных и такое, что $\phi\left(\lambda, f_{0}(z)\right)=f(\lambda, \psi(\lambda, z))$ для всех $(\lambda, z) \in B \times D$.

ДокАзАТЕЛЬСтво. Пусть $\mu$ - характеристика голоморфного движения $\phi$, соответствующая $f_{0}$, и $\psi(\lambda, \cdot)$ - гомеоморфное в $\mathbb{C}$ решение уравнения Бельтрами $\psi_{\bar{z}}=\mu(\lambda, \cdot) \psi_{z}$ с неподвижными точками 0,1 (как и выше, мы предполагаем $\mu$ доопределенной нулем в $\mathbb{C} \backslash D$ ). Такое решение единственное. Положим

$$
\Omega=\{(\lambda, \zeta): \lambda \in B, \zeta \in \psi(\lambda, D)\}
$$

и $f(\lambda, \cdot)=\phi(\lambda, \cdot) \circ f_{0} \circ \psi(\lambda, \cdot)^{-1}$. Так как $\phi$ и $\psi(\lambda, \cdot)$ - гомеоморфизмы, то $f-$ топологическое накрытие.

Фиксируем $\lambda$. Пусть $w$ - голоморфная функция в окрестности точки $p \in M_{\lambda}$ такая, что $d w \mid M_{\lambda} \neq 0$ в этой точке, и $W:=w \circ \phi(\lambda, \cdot) \circ f_{0}$. Тогда $W_{\bar{z}} / W_{z}=$ $\mu(\lambda, \cdot)$ по определению $\mu$ и это же равно $\psi(\lambda, \cdot)_{\bar{z}} / \psi(\lambda, \cdot)_{z}$ по определению $\psi(\lambda, \cdot)$. Поэтому, как и выше, функция $W \circ \psi(\lambda, \cdot)^{-1}=w \circ f(\lambda, \cdot)$ голоморфна в $\Omega_{\lambda}$ и, значит, $f(\lambda, \cdot): \Omega_{\lambda} \rightarrow M_{\lambda}$ - голоморфные накрытия для каждого $\lambda \in B$.

С другой стороны, $\left.S_{z}=\{(\lambda, \psi(\lambda, z)): \lambda \in B)\right\} \subset \Omega$ - голоморфные графики и в каждой точке $(\lambda, \zeta) \in S_{z}$ функция $\psi(\lambda, \cdot)^{-1}$ равна $z$, т. е. сужение $f \mid S_{z}$ равно $\phi\left(\lambda, f_{0}(z)\right)$ и потому голоморфно. Фиксируем произвольную точку $\left(\lambda_{1}, \zeta_{1}\right) \in \Omega$, голоморфный диск $h: \mathbb{D} \rightarrow M, h(0)=\lambda_{1}$ и произвольную голоморфную функцию $g$ в окрестности точки $f\left(\lambda_{1}, \zeta_{1}\right)$ на $M$. Тогда функция $F(\eta, \zeta):=g \circ$ $f(h(\eta), \zeta)$ определена и непрерывна в окрестности точки $\left(0, \zeta_{1}\right) \subset \mathbb{C}^{2}$, голоморфна по $\zeta$ и на каждом графике $S_{z}^{\prime}=\{(h(\eta), \psi(h(\eta), z)): \eta \in \mathbb{D}\}$, пересекающем окрестность этой точки. Так как слои $h(\eta)=$ const и поверхности $S_{z}^{\prime}$ взаимно трансверсальны, то из леммы Шварца легко следует, что $F$ локально удовлетворяет условию Липшица (подробнее см. [16]). По теореме Радемахера, она дифференцируема почти всюду в окрестности $\left(0, \zeta_{1}\right)$ и этот определенный почти всюду дифференциал класса $L_{\text {loc }}^{\infty}$ совпадает с ее дифференциалом в смысле распределений. В точках дифференцируемости $F$ (каковыми являются, например, все точки Лебега для поточечного дифференциала) имеем $\bar{\partial} F=0$, поскольку $F$ голоморфна и на слоях $h(\eta)=$ const, и на $S_{z}^{\prime}$. По лемме Вейля, функция $F$ голоморфна в окрестности $\left(0, \zeta_{1}\right)$. Ввиду произвольности $\lambda_{1}, \zeta_{1}, h$ и $g$, это означает, что $f: \Omega \rightarrow M$ - голоморфное накрытие. Лемма доказана.

Классическое голоморфное движение $\psi: B \times \mathbb{C} \rightarrow \mathbb{C}$ мы называем далее каноническим накрытием движения $\phi$. Отображение $f$ проектирует графики движения $\psi$ в графики движения $\phi$, и, таким образом, “декартово” голоморфное движение $\psi$ является поднятием (прообразом) "криволинейного" голоморфного движения $\phi$. При этом $\psi$ определено на всем $B \times \mathbb{C}$ и дополнительно задает биголоморфное отображение $B \times(\mathbb{C} \backslash \bar{D}) \ni(\lambda, z) \mapsto(\lambda, \psi(\lambda, z)) \in(B \times \mathbb{C}) \backslash \bar{\Omega}$. 
Через $\Phi$ обозначим преобразование $(\lambda, z) \mapsto(\lambda, \psi(\lambda, z))$ пространства $B \times \mathbb{C}$, голоморфное по $\lambda$ и квазиконформное по $z$, которое будем называть каноническим накрытием тривиализации $\phi$ (соответствующим накрытию $f_{0}: D \rightarrow M_{0}$ ); по построению, $\Phi$ есть горизонтально голоморфная тривиализация и так тривиального $B \times \mathbb{C}$ и $f=\phi \circ F_{0} \circ \Phi^{-1}$, где $F_{0}(\lambda, z):=\left(\lambda, f_{0}(z)\right)$.

Построение накрытия $f$ существенно опирается на конкретное голоморфное движение $\phi$, однако фактически $f$ зависит только от накрытия $f_{0}: D \rightarrow M_{0}$.

Лемма 2.7. Пусть $\pi: M \rightarrow B$ - голоморфное семейство римановых поверхностей над комплексным банаховым многообразием $\left(B, \lambda_{0}\right)$ с базовым слоем $M_{0}$ u $f_{0}: D \rightarrow M_{0}$ - голоморфное накрытие областъю $D \subset \mathbb{C}$, не содержащей точек 0,1. Пусть $\phi_{j}: B \times M_{0} \rightarrow M, j=1,2$, - горизонтально голоморфнве тривиализачии $M, \Phi_{j}-$ их поднятия в $B \times D$, соответствующие $f_{0}$, $\Omega_{j}=\Phi_{j}(B \times D)$ u $f_{j}=\phi_{j} \circ F_{0} \circ \Phi_{j}^{-1}: \Omega_{j} \rightarrow M$ - соответствуюшие голоморфные накрытия. Тогда $\Omega_{1}=\Omega_{2}, f_{1} \equiv f_{2}, a \Phi_{1} \equiv \Phi_{2}$ в $B \times(\mathbb{C} \backslash D)$.

ДоказАтеЛьство. Пусть $\mathbb{B} \ni \lambda_{0}-$ координатный шар в $B$ и $D=\bigcup U_{k}-$ покрытие $D$ кругами (с центрами $z_{k}$ ), настолько малыми, что $f_{0} \mid U_{k}$ однолистно для каждого $k$. Положим $V_{k}=\Phi_{1}\left(\mathbb{B} \times U_{k}\right)$. Тогда $V_{k} \subset \Omega_{1}$ и $f_{1} \mid V_{k} \rightarrow f_{1}\left(V_{k}\right)=$ $\phi_{1} \circ F_{0}\left(\mathbb{B} \times U_{k}\right)$ взаимно однозначно.

Так как область $f_{1}\left(V_{k}\right)$ односвязна и $f_{2}$ - накрытие, то существует однозначно определенная область $W_{k} \subset \Omega_{2}$, содержащая $\left(\lambda_{0}, z_{k}\right)$ и такая, что $f_{2} \mid W_{k} \rightarrow$ $f_{1}\left(V_{k}\right)$ однолистно. Таким образом, для всякого $k$ определено голоморфное отображение $g_{k}=\left(f_{2}\right)_{k}^{-1} \circ f_{1}$ с указанной ветвью $f_{2}^{-1}$. Так как $f_{1}=f_{2}$ на $\lambda_{0} \times D$ по условию, то $g_{k}\left(\lambda_{0}, z\right) \equiv\left(\lambda_{0}, z\right)$ для $z \in U_{k}$.

Пусть $U_{k} \cap U_{l}$ непусто. Тогда сужения $\left(f_{2}\right)_{k}^{-1},\left(f_{2}\right)_{l}^{-1}$ на $f_{1}\left(V_{k} \cap V_{l}\right)$ равны, поскольку это поднятия относительно $f_{2}$ односвязной области, совпадающие в $f_{1}\left(\lambda_{0} \times\left(U_{k} \cap U_{l}\right)\right)$. Таким образом, полагая $g=g_{k}$ в $V_{k}$, мы получаем послойный гомеоморфизм $g: \Omega_{1}^{0} \rightarrow \Omega_{2}^{0}$, где $\Omega_{j}^{0}:=\Omega_{j} \cap\left(\pi \circ f_{j}\right)^{-1}(\mathbb{B})$. Так как $f_{2} \circ g=f_{1}$, то отображение $g$ биголоморфное.

Определим в $\mathbb{B} \times \mathbb{C}$ отображение $G$, равное $g \circ \Phi_{1}$ в $\mathbb{B} \times D$ и $\Phi_{2}$ в $B \times(\mathbb{C} \backslash D)$. Тогда $G\left(\lambda_{0}, z\right) \equiv z, G$ инъективно по $z$ и голоморфно по $\lambda$, т. е. $G$ - голоморфное движение, нормированное вместе с $\Phi_{2}$, поскольку $0,1 \notin D$.

Отображение $G \circ \Phi_{1}^{-1}$ такое же; в частности, при каждом фиксированном $\lambda$ оно дифференцируемо почти всюду по $z$, будучи квазиконформным, причем его дифференциал принадлежит классу $L_{\mathrm{loc}}^{2}($ см. $[42])$. Так как $\left(\Phi_{j}(\lambda, \cdot)\right)_{\bar{z}}=0$ почти всюду в $\mathbb{C} \backslash D$, то $\left(\Phi_{2} \circ \Phi_{1}^{-1}(\lambda, \cdot)\right)_{\bar{z}}$ тоже равно 0 почти всюду в $\mathbb{C} \backslash$ $\left(\Omega_{1}\right)_{\lambda}$ (именно, в точках Лебега дифференциала $\left.\Phi_{2} \circ \Phi_{1}^{-1}(\lambda, \cdot)\right)$ при каждом фиксированном $\lambda$. Так как $G \circ \Phi_{1}^{-1}=g$ в $\Omega_{1}^{0}$ голоморфно, то $\left(G \circ \Phi_{1}^{-1}(\lambda, \cdot)\right)_{\bar{z}}=0$ почти всюду в $\mathbb{C}$ при $\lambda \in \mathbb{B}$, и, значит, $G \circ \Phi_{1}^{-1}$ голоморфно по $z$ по лемме Вейля. Так как $G \circ \Phi_{1}^{-1}(\lambda, \cdot)$ инъективно и нормировано, то $G \circ \Phi_{1}^{-1}$ тождественно по $z$, а значит, и в целом. В частности, оно тождественное на $\Omega_{1}^{0}$ и на $(B \times \mathbb{C}) \backslash \Omega_{1}^{0}$, где равно соответственно $g$ и $\Phi_{2} \circ \Phi_{1}^{-1}$.

Таким образом, доказано, что $\Omega_{1}^{0}=\Omega_{2}^{0}, f_{1}=f_{2}$ на $\Omega_{1}^{0}$ и $\Phi_{1}=\Phi_{2}$ на $\mathbb{B} \times(\mathbb{C} \backslash D)$. Так как $\Phi_{j}$ голоморфны по $\lambda \in B$, то, по теореме единственности, $\Phi_{1}=\Phi_{2}$ в $B \times(\mathbb{C} \backslash D)$, из чего следует, в частности, что $\Omega_{1}=\Omega_{2}$. А тогда $f_{1}=f_{2}$ по теореме единственности. Лемма доказана. 
Отображение $f: \Omega \rightarrow M$ мы называем далее каноническим накрытием семейства $\pi: M \rightarrow B$ (соответствующим накрытию $f_{0}$ ), а если $D=\mathbb{D}$, то однозначно определенное по лемме 2.7 отображение $f: \Omega \rightarrow M$ назовем канонической униформизацией семейства $M$. Из конструкции в лемме 2.6 очевидно следует, что все слои прообраза канонической униформизации являются квазикругами, а дополнение к $\bar{\Omega}$ в $B \times \mathbb{C}$ биголоморфно послойно эквивалентно $B \times(\mathbb{C} \backslash \overline{\mathbb{D}})$, в частности, оно заполняется голоморфным движением (голоморфным по совокупности переменных!). По $\lambda$-лемме 2.4 это движение продолжается до голоморфного движения множества $\mathbb{C} \backslash \mathbb{D}$ над $\left(B, \lambda_{0}\right)$, и, значит, граница $\Omega$ заполняется голоморфным движением окружности $\partial \mathbb{D}$ над $\left(B, \lambda_{0}\right)$, т. е. является объединением однопараметрического семейства попарно непересекающихся голоморфных графиков над $B$.

Условие $D \not \supset 0,1$ в лемме 2.7 существенно не только для доказательства. Например, для $D=\widehat{\mathbb{C}}, \mathbb{C}$ или $\mathbb{C} \backslash 0$ различные голоморфные движения $B \times D \rightarrow$ $M=B \times D$ порождают, вообще говоря, различные отображения $f$. (Доказательство не проходит потому, что отображение $G$ в этом случае не обязательно нормированное, условия $0,1 \notin D$ нет.) Пусть, например, $\phi_{1}$ - тождественное отображение $M=B \times D$ с такой $D, f_{0}: z \mapsto\left(\lambda_{0}, z\right)$ (тогда $f_{1}$ тоже тождественное) и $\alpha: B \times D \rightarrow D$ - голоморфное отображение, которое при каждом фиксированном $\lambda \in B$ является автоморфизмом $D$, причем $\alpha\left(\lambda_{0}, z\right) \equiv z$, но в целом $\alpha(\lambda, z) \not \equiv z$. Тогда $\phi_{2}:(\lambda, z) \mapsto(\lambda, \alpha(\lambda, z))$ - голоморфное движение, заполняющее $M$. Так как оно голоморфно по $z$, то $\Phi_{2}$ - тождественное отображение, и, значит, $f_{2}=\phi_{2} \circ \Phi_{2}^{-1}-$ нетождественный автоморфизм $M$. Для совпадения накрытий $f_{1}, f_{2}$, соответствующих различным голоморфным движениям и одинаковому накрытию $f_{0}: D \rightarrow M_{0}$, при $D=\mathbb{C} \backslash 0$ достаточно, чтобы $\phi_{1}(\cdot, p)=\phi_{2}(\cdot, p)$ для какого-нибудь одного значения $p \in M_{0}$, для $D=\mathbb{C}$ нужно совпадение уже двух сечений, а для $D=\widehat{\mathbb{C}}-$ трех.

2.3. Униформизация локально тривиализуемых семейств. Свойство единственности канонических накрытий позволяет воспользоваться стандартным методом аналитического продолжения для униформизации семейств, локально тривиализуемых голоморфными движениями.

Теорема 2.1. Пусть $\pi: M \rightarrow B$ - голоморфное семейство гиперболических римановых поверхностей над односвязным комплексным банаховым многообразием $\left(B, \lambda_{0}\right)$ и $f_{0}: D \rightarrow M_{0}$ - голоморфное накрытие областью $D \subset \mathbb{C}$. Предположим, что существует покрытие $B$ областями $B_{\alpha}$ такими, что каждое $\pi^{-1}\left(B_{\alpha}\right)$ заполняется некоторым голоморфным движением. Тогда существуют область $\Omega \subset B \times \mathbb{C}, \Omega_{0}=D$, и сохраняющее проекцию голоморфное накрьтие (униформизачия) $f: \Omega \rightarrow M, f\left(\lambda_{0}, \cdot\right)=f_{0}$. При этом $(B \times \mathbb{C}) \backslash \bar{\Omega}$ послойно биголоморфно эквивалентно $B \times(\mathbb{C} \backslash \bar{D})$, и если $D=\operatorname{int} \bar{D}$, то граница $\Omega$ в $B \times \mathbb{C}$ заполняется однозначно определенным голоморфным движением над $\left(B, \lambda_{0}\right)$.

ДокАзАТЕльство. Так как $D$ - гиперболическая область, то без ограничения общности можно считать, что $0,1 \notin D$.

Пусть $\lambda(t), t \in[0,1],-$ путь в $B$ с концами $\lambda_{0}, \lambda_{1}$. Тогда существуют $0=$ $t_{0}<t_{1}<\cdots<t_{N}=1$ такие, что $\lambda\left(\left[t_{\nu}, t_{\nu+1}\right]\right) \subset B^{\nu}:=B_{\alpha_{\nu}}$ для некоторых $\alpha_{\nu}$, $\nu=0, \ldots, N-1$. По условию и лемме 2.6 , существуют голоморфное движение 
$\phi_{0}: B^{0} \times M_{0} \rightarrow \pi^{-1}\left(B^{0}\right)$, область $\Omega^{0} \in B^{0} \times \mathbb{C}, \Omega_{\lambda_{0}}^{0}=\lambda_{0} \times D$, и каноническое накрытие $f^{0}: \Omega^{0} \rightarrow \pi^{-1}\left(B^{0}\right)$ с $f^{0}\left(\lambda_{0}, z\right)=f_{0}(z), z \in D$.

Область $D_{1}$ определим из условия $\Omega_{\lambda\left(t_{1}\right)}^{0}=\lambda\left(t_{1}\right) \times D_{1}$ и положим $M_{1}=M_{\lambda\left(t_{1}\right)}$ и $f_{1}=\phi_{0}\left(\lambda\left(t_{1}\right), \cdot\right) \circ f_{0} \circ \psi_{0}\left(\lambda\left(t_{1}\right), \cdot\right)^{-1}: D_{1} \rightarrow M_{1}$. Опять по условию и лемме 2.6 существуют голоморфное движение $\phi_{1}: B^{1} \times M_{1} \rightarrow \pi^{-1}\left(B^{1}\right)$, его каноническое накрытие $\psi_{1}: B^{1} \times \mathbb{C} \rightarrow \mathbb{C}$ и каноническое накрытие $f^{1}: \Omega^{1} \rightarrow \pi^{-1}\left(B^{1}\right)$ семейства $\pi^{-1}\left(B^{0}\right)$ областью $\Omega^{1} \subset B^{1} \times \mathbb{C}$, которое по лемме 2.7 однозначно определяется условием, что $f^{1}=f_{1}$ на $\Omega_{\lambda\left(t_{1}\right)}^{0}$. По лемме 2.6,

$f^{1}(\lambda, \cdot)=\phi_{1}(\lambda, \cdot) \circ f_{1} \circ \psi_{1}(\lambda, \cdot)^{-1}=\phi_{1}(\lambda, \cdot) \circ \phi_{0}\left(\lambda\left(t_{1}\right), \cdot\right) \circ f_{0} \circ \psi_{0}\left(\lambda\left(t_{1}\right), \cdot\right)^{-1} \circ \psi_{1}(\lambda, \cdot)^{-1}$.

Продолжая так по индукции, получаем области $\Omega^{\nu} \subset B^{\nu} \times \mathbb{C}$, и послойные биголоморфизмы $f^{\nu}: \Omega^{\nu} \rightarrow \pi^{-1}\left(B^{\nu}\right)$, причем $f^{\nu}=f^{\nu-1}$ на $\Omega_{\lambda\left(t_{\nu}\right)}^{\nu-1}=\lambda\left(t_{\nu}\right) \times D_{\nu}$ и $\Omega_{\lambda}^{\nu-1}=\Omega_{\lambda}^{\nu}$ для всех $\lambda$ из связной компоненты $B^{\nu-1} \cap B^{\nu}$, содержащей $\lambda\left(t_{\nu}\right)$, $\nu=1, \ldots, N$. По построению, $f^{\nu}(\lambda, \cdot)=\tilde{\phi}_{\nu}(\lambda, \cdot) \circ f_{0} \circ \tilde{\psi}_{\nu+1}(\lambda, \cdot)^{-1}$, где

$$
\begin{aligned}
& \tilde{\phi}_{\nu}(\lambda, \cdot)=\phi_{\nu}(\lambda, \cdot) \circ \phi_{\nu-1}\left(\lambda\left(t_{\nu}\right), \cdot\right) \circ \cdots \circ \phi_{0}\left(\lambda\left(t_{1}\right), \cdot\right): M_{0} \rightarrow M_{\lambda}, \\
& \tilde{\psi}_{\nu}(\lambda, \cdot)=\psi_{\nu}(\lambda, \cdot) \circ \psi_{\nu-1}\left(\lambda\left(t_{\nu}\right), \cdot\right) \circ \cdots \circ \psi_{0}\left(\lambda\left(t_{1}\right), \cdot\right): D \rightarrow \Omega_{\lambda} .
\end{aligned}
$$

Положим $\Omega=\bigcup \Omega^{\nu}$ и $f \mid \Omega^{\nu}:=f^{\nu}$. Тогда результат продолжения по цепочке, $f \mid \Omega^{N}$, не зависит от выбора $\left\{t_{\nu}\right\}$ : если $\left\{\tau_{\mu}\right\}-$ другой такой набор, то упорядочивая $\left\{t_{\nu}\right\} \cup\left\{\tau_{\mu}\right\}$ и повторяя описанный выше процесс продолжения для этого более мелкого разбиения $\left\{t_{j}^{\prime}\right\} \subset[0,1]$, по теореме единственности (лемме 2.7) получаем, что множество точек $t \in[0,1]$ таких, что $\Omega_{\lambda(t)}=\Omega_{\lambda(t)}^{\prime}$ и $f\left|\Omega_{\lambda(t)}=f^{\prime}\right| \Omega_{\lambda(t)}$, открытое и замкнутое на $[0,1]$.

Далее, если $\lambda^{\prime}(t)$ - другой путь, соединяющий $\lambda_{0}$ с $\lambda_{1}$ и достаточно близкий к $\lambda(t)$, то точки $t_{\nu}^{\prime}$ можно брать так, что $\lambda\left(t_{\nu}\right)$ и $\lambda^{\prime}\left(t_{\nu}^{\prime}\right)$ лежат в одной связной компоненте $U_{\nu}$ множества $B^{\nu-1} \cap B^{\nu}$, и тогда по теореме единственности $f\left|\Omega^{\nu}=f^{\prime}\right| \Omega^{\nu}$, где $f^{\prime}$ - продолжение $f_{0}$ вдоль пути $\lambda^{\prime}(t)$. Так как $B$ односвязно, то отсюда, как в стандартной теореме о монодромии, получаем однозначно определенное накрытием $f_{0}$ голоморфное послойное накрытие $f: \Omega \rightarrow M$ областью $\Omega \subset B \times \mathbb{C}$.

По построению, $f(\lambda, \cdot)$ для $\lambda \in B_{\alpha}$ представляется в виде $\tilde{\phi}_{\alpha}(\lambda, \cdot) \circ f_{0} \circ$ $\tilde{\psi}_{\alpha}(\lambda, \cdot)^{-1}$, причем квазиконформные отображения $\tilde{\psi}_{\alpha}(\lambda, \cdot)$, вообще говоря, зависят от $\alpha$. Однако $\tilde{\psi}_{\alpha}(\lambda, \cdot), \lambda \in B_{\alpha}$, определены на всей плоскости $\mathbb{C}$ и в $\mathbb{C} \backslash D$ они от $\alpha$ не зависят. В самом деле, аналитически продолжая $f^{0}$, мы одновременно продолжаем и каноническое накрытие $\psi_{0} \mid B^{0} \times(\mathbb{C} \backslash \bar{D}) \rightarrow \mathbb{C}$, хотя и определяемое движением $\phi_{0}$, но зависящее только от $f_{0}$ по лемме 2.7. По определению, $\psi_{0}(\lambda, \cdot): \mathbb{C} \backslash D \rightarrow \mathbb{C} \backslash \Omega_{\lambda}^{0}, \lambda \in B^{0}$. Для пути $\lambda(t)$ с концами $\lambda_{0}$ и $\lambda_{1}$ и $0=t_{0}<\cdots<t_{N}=1$, как выше, отображение $\psi_{\nu}(\lambda, \cdot): \mathbb{C} \backslash D_{\nu} \rightarrow \mathbb{C} \backslash \Omega_{\lambda}$, $\lambda \in B^{\nu}$, однозначно определяется условием, что $\psi_{\nu}\left(\lambda\left(t_{\nu}\right), \cdot\right)=\psi_{\nu-1}\left(\lambda\left(t_{\nu}\right), \cdot\right)$ на $\mathbb{C} \backslash D_{\nu}$.

Положим $\tilde{\psi}_{0}=\psi_{0}$ на $B^{0} \times(\mathbb{C} \backslash \bar{D})$ и, по индукции, $\tilde{\psi}_{\nu}(\lambda, z):=\psi_{\nu}(\lambda, \cdot) \circ$ $\tilde{\psi}_{\nu-1}\left(\lambda\left(t_{\nu}, \cdot\right)\right)$ для $\lambda \in B^{\nu}$. По лемме 2.7 и теореме о монодромии, как выше, в результате такого аналитического продолжения получается биголоморфное отображение $B \times(\mathbb{C} \backslash \bar{D}) \rightarrow(B \times \mathbb{C}) \backslash \bar{\Omega}$. Если $D=\operatorname{int} \bar{D}$, то по $\lambda$-лемме 2.4 оно продолжается до голоморфного движения $\tilde{\psi}$ множества $\mathbb{C} \backslash D$ над $\left(B, \lambda_{0}\right)$, в частности, граница $\Omega$ в $B \times \mathbb{C}$ заполняется голоморфным движением $\tilde{\psi} \mid B \times \partial D$. Теорема доказана. 
ДоПолнение 2.1. Пусть Г есть накрывающая группа накрытия $f_{0}$. Тогда накрывающая группа $G$ накрытия $f$ в каждом слое $\Omega_{\lambda}$ действует как $w_{\lambda} \Gamma w_{\lambda}^{-1}$, где $w_{\lambda}: D \rightarrow \Omega_{\lambda}-$ квазиконформное отображение, причем коэффициент Бельтрами $\mu(\lambda, \cdot):=\left(w_{\lambda}\right)_{\bar{z}} /\left(w_{\lambda}\right)_{z}$ инвариантен относительно $\Gamma$, точнее, $(\mu(\lambda, \cdot) \circ A) \overline{A^{\prime}} / A^{\prime}=\mu(\lambda, \cdot)$ для всех $A \in \Gamma$. Элементы $G_{\lambda}$ являются дробно-линейными отображениями и, таким образом, $G$ продолжается до подгруппы голоморфных послойнъх автоморфизмов многообразия $B \times \widehat{\mathbb{C}}$.

ДокАЗАТЕЛЬСтво. По построению, $f(\lambda, \cdot)=\phi_{0}(\lambda, \cdot) \circ f_{0} \circ \psi_{0}^{-1}(\lambda, \cdot)$ для $\lambda \in B_{0}$. Так как $f G=f$ и $\phi_{0}$ - гомеоморфизм, то $\left(f_{0} \circ \psi_{0}(\lambda, \cdot)^{-1}\right) G_{\lambda}=f_{0} \circ \psi_{0}(\lambda, \cdot)^{-1}$. Отсюда $f_{0}\left(\psi_{0}(\lambda, \cdot)^{-1} G_{\lambda} \psi_{0}(\lambda, \cdot)\right)=f_{0}$, т. е. $\psi_{0}(\lambda, \cdot)^{-1} G_{\lambda} \psi_{0}(\lambda, \cdot) \subset \Gamma$, и потому $G_{\lambda} \subset \psi_{0}(\lambda, \cdot) \Gamma \psi_{0}(\lambda, \cdot)^{-1}$ для $\lambda \in B_{0}$.

Так как $\psi_{0}(\lambda, \cdot)$ - решение уравнения Бельтрами с коэффициентом $\mu_{0}(\lambda, \cdot)$, инвариантным относительно $\Gamma$, то для всякого $A \in \Gamma$ функция $\psi_{0}(\lambda, \cdot) \circ A$ - тоже решение этого уравнения, не обязательно нормированное, точки $0,1, \infty$ не фиксированы. Так как нормированное решение единственное, то существует дробно-линейное отображение $C_{\lambda}$ такое, что $\psi_{0}(\lambda, \cdot) \circ A=C_{\lambda} \circ \psi_{0}(\lambda, \cdot)$ и, значит, $\psi_{0}(\lambda, \cdot) A \psi_{0}(\lambda, \cdot)^{-1}=C_{\lambda}$ для $\lambda \in B_{0}$. Таким образом, при $\lambda \in B_{0}$ элементы группы $\psi_{0}(\lambda, \cdot) \Gamma \psi_{0}(\lambda, \cdot)^{-1}$, содержащей $G_{\lambda}$, являются дробно-линейными отображениями.

Производная Шварца любой дробно-линейной функции равна нулю, поэтому из голоморфности $G$ и теоремы единственности следует, что все группы $G_{\lambda}$ состоят из дробно-линейных отображений. Голоморфность элементов $G$ означает, что локально (по $\lambda$ ) коэффициенты этих отображений можно выбирать голоморфными по $\lambda$ и, таким образом, элементы $G$ продолжаются до голоморфных послойных автоморфизмов $B \times \widehat{\mathbb{C}}$.

По построению $f$, отображение $f(\lambda, \cdot)$ для $\lambda \in B_{\alpha}$ равно $\tilde{\phi}_{\alpha}(\lambda, \cdot) \circ f_{0} \circ \tilde{\psi}_{\alpha}(\lambda, \cdot)^{-1}$, где $\tilde{\psi}_{\alpha}(\lambda, \cdot) \mid D \rightarrow \Omega_{\lambda}$. Поэтому, как выше, $f_{0} \circ \tilde{\psi}_{\alpha}(\lambda, \cdot)^{-1} G_{\lambda}=f_{0} \circ \tilde{\psi}_{\alpha}(\lambda, \cdot)^{-1}$, откуда $G_{\lambda} \subset w_{\lambda} \Gamma w_{\lambda}^{-1}$, где $w_{\lambda}:=\tilde{\psi}_{\alpha}(\lambda, \cdot)$. По определению,

$$
w_{\lambda}=\psi_{N}(\lambda, \cdot) \circ \psi_{N-1}\left(\lambda\left(t_{N}\right), \cdot\right) \circ \cdots \circ \psi_{0}\left(\lambda\left(t_{1}\right), \cdot\right)
$$

(из процесса аналитического продолжения). Положим $G^{\nu}=G_{\lambda\left(t_{\nu}\right)}$. Тогда по индукции, как выше, $G^{\nu+1} \subset \psi_{\nu}\left(\lambda\left(t_{\nu+1}\right), \cdot\right) G^{\nu} \psi_{\nu}\left(\lambda\left(t_{\nu+1}\right), \cdot\right)^{-1}$ и, значит, для любого $A \in \Gamma$ найдется дробно-линейное преобразование $C_{\lambda}$ такое, что $w_{\lambda} \circ A=$ $C_{\lambda} \circ w_{\lambda}$. Из этого очевидно следует, что $(\mu(\lambda, \cdot) \circ A) \overline{A^{\prime}} / A^{\prime}=\mu(\lambda, \cdot)$ для $A \in \Gamma$.

Наконец, для каждого $\lambda \in B$ дробно-линейные преобразования $w_{\lambda} \circ A \circ w_{\lambda}^{-1}$, $A \in \Gamma$, принадлежат накрывающей группе накрытия $f(\lambda, \cdot): \Omega_{\lambda} \rightarrow M_{\lambda}$. Так как они голоморфно зависят от $\lambda$, то из этого следует, что они принадлежат $G_{\lambda}$ и, значит, $G_{\lambda}=w_{\lambda} \Gamma w_{\lambda}^{-1}$ для всякого $\lambda \in B$.

ЗАмечАниЕ 2.1. Отображения $w_{\lambda}$ над каждым $B_{\alpha}$ можно брать голоморфно зависящими от $\lambda$ (см. определение). В целом они определены по $\lambda$ не однозначно, но их сужения на $\mathbb{C} \backslash D$ и группы $w_{\lambda} \Gamma w_{\lambda}^{-1}$ по доказанному выше не зависят от выбора $w_{\lambda}$ (соответствующего выбору $B_{\alpha} \ni \lambda$ ), а зависят только от $\lambda$.

ЗАмЕчАНИЕ 2.2. Несмотря на то, что граница $\Omega$ заполняется голоморфным движением, сама область $\Omega$ может не содержать ни одного глобального голоморфного сечения проекции в $B$. Типичный пример - слоения Берса над 
пространствами Тейхмюллера гиперболических римановых поверхностей (см. ниже).

ЗАмечание 2.3. Условие локального заполнения семейства $\pi: M \rightarrow B$ голоморфными движениями является основным для определения "маркировки" такого семейства по Эрлу-Фоулеру [20]. В общем для маркировки нужно еще условие согласования локальных голоморфных движений над попарными пересечениями $B_{\alpha} \cap B_{\beta}$, но для односвязной базы $B$ оно излишнее [20; теорема 4].

Лемма 2.8. Пусть $\pi: M \rightarrow B$ - голоморфное семейство гиперболических римановых поверхностей, $f_{j}: D_{j} \rightarrow M_{0}, j=0,1$, - голоморфные накрытия $u h: D_{1} \rightarrow D_{0}$ - голоморфное отображение такое, что $f_{1}=f_{0} \circ h$. Пусть $f^{j}: \Omega^{j} \rightarrow M$ - соответствующие канонические накрытия. Тогда существует голоморфное послойное накрытие $g: \Omega^{1} \rightarrow \Omega^{0}$ maкое, что $g\left(\lambda_{0}, \cdot\right)=h u f^{1}=$ $f^{0} \circ g$.

ДоказАТЕЛЬСтво. Пусть $G^{j}$ - накрывающие группы накрытий $f^{j}, B=$ $\bigcup B_{\alpha}, \phi_{\alpha}: B_{\alpha} \times M_{\lambda_{\alpha}} \rightarrow \pi^{-1}\left(B_{\alpha}\right)$ - горизонтальные голоморфные тривиализации и $\psi_{\alpha}^{j}: B_{\alpha} \times \Omega_{\lambda_{\alpha}}^{j} \rightarrow \Omega_{\alpha}^{j}-$ их канонические накрытия. Тогда $f^{j}(\lambda, \cdot)=\tilde{\phi}_{\alpha}(\lambda, \cdot) \circ$ $f_{j} \circ \tilde{\psi}_{\alpha}^{j}(\lambda, \cdot)^{-1}$ для $\lambda \in B_{\alpha}$ (см. доказательство теоремы 2.1) и, значит,

$$
f^{1}(\lambda, \cdot) \circ \tilde{\psi}_{\alpha}^{1}(\lambda, \cdot)=\tilde{\phi}_{\alpha} \circ f_{1}=\tilde{\phi}_{\alpha} \circ f_{0} \circ h=f^{0}(\lambda, \cdot) \circ \tilde{\psi}_{\alpha}^{0}(\lambda, \cdot) \circ h,
$$

т. е. $f^{1}=f^{0} \circ g_{\alpha}$, где $g_{\alpha}(\lambda, \cdot):=\tilde{\psi}_{\alpha}^{0}(\lambda, \cdot) \circ h \circ \tilde{\psi}_{\alpha}^{1}(\lambda, \cdot)^{-1}: \Omega_{\lambda}^{1} \rightarrow \Omega_{\lambda}^{0}, \lambda \in B_{\alpha}$, причем $g_{\alpha}$ голоморфно в $\Omega_{\alpha}^{1}$ по совокупности переменных.

Отсюда $f^{0} \circ g_{\alpha}=f^{0} \circ g_{\beta}$ в $\Omega_{\alpha}^{1} \cap \Omega_{\beta}^{1}$.

Предположим, что $g_{\alpha}\left(\lambda^{\prime}, \cdot\right)=g_{\beta}\left(\lambda^{\prime}, \cdot\right)$ для некоторого $\lambda^{\prime} \in B_{\alpha} \cap B_{\beta}$. Тогда для фиксированного $c \in \Omega_{\lambda^{\prime}}^{1}$ из равенства $f^{0} \circ g_{\alpha}(\lambda, c)=f^{0} \circ g_{\beta}(\lambda, c)$ для $\lambda$, близких к $\lambda^{\prime}$, следует, что существует $A_{c}(\lambda, \cdot) \in G_{\lambda}^{0}$ такое, что $g_{\beta}(\lambda, c)=A_{c}(\lambda, \cdot) \circ g_{\alpha}(\lambda, c)$. Так как $g_{\beta}(\lambda, \cdot) \rightarrow g_{\alpha}\left(\lambda^{\prime}, \cdot\right)$ при $\lambda \rightarrow \lambda^{\prime}$, то $A_{c}(\lambda, \cdot) \rightarrow c$. Так как элементы $G_{\lambda^{\prime}}^{0}$ не имеют неподвижных точек в $\Omega_{\lambda^{\prime}}^{0}$, то $A_{c}(\lambda, \cdot) \rightarrow$ id при $\lambda \rightarrow \lambda^{\prime}$. Так как группа $G^{0}$ дискретная, то из этого следует, что $A_{c}(\lambda, \cdot)=\mathrm{id}$ при $\lambda$, близких к $\lambda^{\prime}$. Отсюда и из теоремы единственности получаем, что $g_{\alpha}=g_{\beta}$ в связной компоненте множества $\Omega_{\alpha}^{1} \cap \Omega_{\beta}^{1}$, содержащей $\Omega_{\lambda^{\prime}}^{1}$.

Имея такую единственность, мы можем аналитически продолжать элемент $g_{0}$, определенный над $B_{0}$, по любому пути в $B$, как это описано в доказательстве теоремы 2.1. Так как $B$ односвязно, то в результате получится глобальное голоморфное послойное отображение $g: \Omega^{1} \rightarrow \Omega^{0}$. По теореме единственности, $f^{1}=f^{0} \circ g$, так как это верно над $B_{0}$. Лемма доказана.

\section{3. Голоморфные движения и пространства Тейхмюллера}

3.1. Универсальные семейства. Пусть $\Gamma \subset$ Aut $\mathbb{D}$ - фуксова группа, т. е. дискретная подгруппа дробно-линейных автоморфизмов круга. Всюду в дальнейшем предполагаем, что Г не имеет кручения (это эквивалентно тому, что элементы группы $Г$ не имеют неподвижных точек в $\mathbb{D}$ ). Тогда $\mathbb{D} / \Gamma$ - риманова поверхность, факторотображение $\mathbb{D} \rightarrow \mathbb{D} / \Gamma$ является универсальным накрытием с накрывающей группой $\Gamma$, изоморфной фундаментальной группе $\pi_{1}(\mathbb{D} / \Gamma)$. По теореме Пуанкаре-Кёбе, всякая риманова поверхность конформно эквивалентна поверхности вида $\mathbb{D} / \Gamma$ для некоторой фуксовой группы Г без кручения. 
Обозначим через $L^{\infty}(\mathbb{D}, \Gamma)$ замкнутое подпространство в $L^{\infty}(\mathbb{D})$, состоящее из функций $\mu$ таких, что

$$
(\mu \circ A) \overline{A^{\prime}} / A^{\prime}=\mu
$$

для всех $A \in \Gamma$. Единичный шар в $L^{\infty}(\mathbb{D}, \Gamma)$ обозначим $\mathscr{M}_{1}(\Gamma)$. Өлементы $\mu \in \mathscr{M}_{1}(\Gamma)$ продолжаем нулем в $\mathbb{C} \backslash \mathbb{D}$, не меняя обозначений, и, таким образом, считаем, что $\mathscr{M}_{1}(\Gamma) \subset L^{\infty}(\mathbb{C})$.

По теореме Боярского [13], [14], для всякой $\mu \in \mathscr{M}_{1}(\Gamma)$ существует единственное гомеоморфное в $\mathbb{C}$ решение $w^{\mu}$ уравнения Бельтрами $w_{\bar{z}}=\mu w_{z}$, нормированное условием $w^{\mu}(z)=z+o(1)$ в окрестности $\infty$ (решение в классе Соболева $W_{\text {loc }}^{1, p}$ с некоторым $p>2$, зависящим от $\|\mu\|_{\infty}$, см. ниже п. 4.1). Два элемента $\mu_{1}, \mu_{2}$ считаются эквивалентными по Тейхмюллеру, если $w^{\mu_{1}}=w^{\mu_{2}}$ на $\partial \mathbb{D}($ а тогда и на $\mathbb{C} \backslash \mathbb{D})$. Факторпространство $\mathscr{M}_{1}(\Gamma)$ по этому отношению эквивалентности называется пространством Тейхмюллера фуксовой группы $\Gamma$ и обозначается $T(\Gamma)$. Это в общем случае бесконечномерное комплексное банахово многообразие, комплексная структура которого однозначно определяется условием голоморфности факторотображения $\Phi: \mathscr{M}_{1}(\Gamma) \rightarrow T(\Gamma)$.

Согласно общей теории пространств Тейхмюллера (см. [26], [41], [47], [17]), $T(\Gamma)$ биголоморфно эквивалентно области в банаховом пространстве $\mathscr{B}_{2}(\Gamma)$ квадратичных дифференциалов на $\widehat{\mathbb{C}} \backslash \overline{\mathbb{D}}$, т. е. голоморфных в $\widehat{\mathbb{C}} \backslash \overline{\mathbb{D}}$ функций $\tau$ таких, что

$$
(\tau \circ A)\left(A^{\prime}\right)^{2}=\tau, \quad A \in \Gamma,
$$

и норма $\|\tau\|:=(1 / 6) \sup _{\mathbb{C} \backslash \overline{\mathbb{D}}}\left(\left|z^{2}\right|-1\right)^{2}|\tau(z)|$ конечна (коэффициент $1 / 6$ - для удобной нам нормировки). Этот биголоморфизм переводит класс Тейхмюллера $[\mu]$ в производную Шварца $S w^{\mu} \in \mathscr{B}_{2}(\Gamma)$ (не зависящую от представителя $\mu \in[\mu])$. По теореме Крауса [39] (Нехари [48]) образ $T(\Gamma)$ содержится в единичном шаре пространства $\mathscr{B}_{2}(\Gamma)$ и по теореме Альфорса ([1; VI C1]) содержит шар $\|\tau\|<1 / 3$. Всюду далее мы отождествляем $T(\Gamma)$ с этой областью в $\mathscr{B}_{2}(\Gamma)$.

По определению классов Тейхмюллера, область $w^{\mu}(\mathbb{D})$ зависит только от класса $[\mu]$ функции $\mu \in \mathscr{M}_{1}(\Gamma)$ и, таким образом, в $T(\Gamma) \times \mathbb{C}$ естественно определяется область

$$
F(\Gamma)=\left\{([\mu], z): z \in w^{\mu}(\mathbb{D})\right\}
$$

с голоморфной проекцией $([\mu], z) \mapsto[\mu]$, превращающей $F(\Gamma)$ в голоморфное семейство плоских жордановых областей. Голоморфное семейство $F(\Gamma) \rightarrow T(\Gamma)$ называется расслоением Берса фуксовой группы Г (см. [10], [47]).

Дробно-линейные преобразования $A \subset \Gamma$ действуют на $F(\Gamma)$ по правилу

$$
([\mu], z) \mapsto\left([\mu], w^{\mu} \circ A \circ\left(w^{\mu}\right)^{-1}(z)\right)
$$

(отображения $w^{\mu} \circ A \circ\left(w^{\mu}\right)^{-1}$ оказываются дробно-линейными и не зависящими от $\mu$ из одного класса; см. дополнение 2.1 к теореме 2.1 или [1; VI A]). Это действие послойное и голоморфное. Фактор $V(\Gamma)$ многообразия $F(\Gamma)$ по этому действию Г определяет голоморфное семейство римановых поверхностей $V(\Gamma)_{[\mu]}:=F(\Gamma)_{[\mu]} / w^{\mu} \circ \Gamma \circ\left(w^{\mu}\right)^{-1}$ над $T(\Gamma)$, которое называют также универсальной кривой Тейхмюллера (фуксовой группы Г). Пространства Тейхмюллеpa, как известно, односвязные (и даже стягиваемые), поэтому $F(\Gamma) \rightarrow V(\Gamma)-$ универсальное накрытие над $V(\Gamma)$. Поскольку оно еще и послойное и $F(\Gamma) \subset$ 
$T(\Gamma) \times \mathbb{C}$, то факторпроекция в $V(\Gamma)$ является униформизацией голоморфного семейства $V(\Gamma) \rightarrow T(\Gamma)$.

Проекция $\mathscr{M}_{1}(\Gamma) \rightarrow T(\Gamma)$ допускает голоморфные сечения в окрестности каждой точки $\tau \in T(\Gamma)$ (что не очевидно ввиду бесконечной размерности "большинства" пространств $T(\Gamma))$. Если $\tau \mapsto \mu(\tau, \cdot)$ - такое сечение $\Phi$ над $U \subset T(\Gamma)$, то, по теореме Альфорса-Берса [1], [2], функции $w^{\mu(\tau, \cdot)}(z)$ на $U \times \mathbb{D}$ голоморфно зависят от $\tau$ при каждом фиксированном $z \in \mathbb{D}$ (будучи всего лишь квазиконформными при каждом фиксированном $\tau \in U)$. Голоморфные сечения $\left(\tau, w^{\mu(\tau, \cdot)}(z)\right), z \in \mathbb{D}$, проекции $F(\Gamma) \rightarrow T(\Gamma)$ заполняют $F(\Gamma) \cap\{\tau \in U\}$ и тем самым задают горизонтально голоморфную тривиализацию $F(\Gamma)$ над $U$ (с произвольным базовым слоем $\left.F(\Gamma)_{\tau^{0}}, \tau^{0} \in U\right)$.

Накрывающая группа проекции $F(\Gamma) \rightarrow V(\Gamma)$ в слое $F(\Gamma)_{\tau}$ действует как $w^{\mu(\tau, \cdot)} \Gamma\left(w^{\mu(\tau, \cdot)}\right)^{-1}$ (см. конец п. 2.3). Пусть $A \in \Gamma$ и $B$ - элемент накрывающей группы, действующий в слоях как $B_{\tau}=w^{\mu(\tau, \cdot)} A\left(w^{\mu(\tau, \cdot)}\right)^{-1}$. Тогда $B_{\tau} \circ w^{\mu(\tau, \cdot)}=$ $w^{\mu(\tau, \cdot)} \circ A$, т. е. $B$ переводит сечение $w=w^{\mu(\tau, \cdot)}(z)$ в сечение $w=w^{\mu(\tau, \cdot)}(A(z))$ из того же голоморфного движения. Таким образом, построенная горизонтально голоморфная тривиализация $F(\Gamma)$ над $U$ инвариантна относительно действия накрывающей группы, и потому ее факторотображение задает горизонтально голоморфную тривиализацию $V(\Gamma)$ над $U$.

В частности, над окрестностью базовой точки $[0] \in T(\Gamma)$ есть замечательное сечение

$$
\tau \mapsto \mu(\tau, z)=\frac{\left(1-|z|^{2}\right)^{2} \tau(1 / \bar{z})}{\bar{z}^{4}} \in \mathscr{M}_{1}(\Gamma)
$$

определенное и голоморфное (по $\tau$ ) в шаре $\{\|\tau\|<1 / 3\} \subset \mathscr{B}_{2}(\Gamma)$ (функции $\tau \in \mathscr{B}_{2}(\Gamma)$ имеют в $\infty$ нуль порядка не меньше 4). Следуя [12], эти $\mu(\tau, \cdot)$ мы называем гармоническими коэффичиентами Бельтрами. Это действительно сечение, так как производная Шварца от $w^{\mu(\tau, \cdot)}(z)$ в $\mathbb{C} \backslash \overline{\mathbb{D}}$ равна $\tau$ (лемма Альфорса-Вейля [3]). Таким образом, расслоение Берса $F(\Gamma)$ горизонтально голоморфно тривиализуется над шаром $T_{1 / 3}(\Gamma):=\{\|\tau\|<1 / 3\}$. Так как эта тривиализация инвариантна относительно действия Г (орбиты переходят в орбиты), то корректно определена ее проекция в $V(\Gamma)$ и соответствующее голоморфное движение мы тоже называем гармоническим.

Все эти тривиализации голоморфны лишь по $\tau$, а по $z$ только квазиконформны. Однако вне $\overline{\mathbb{D}}$ все функции $w^{\mu}$ голоморфны по $z$ и зависят только от $[\mu]$. Так как локально представителей $\mu \in[\mu]$ можно выбирать голоморфно зависящими от $\tau$, то дополнение к $F(\Gamma)$ в $T(\Gamma) \times \widehat{\mathbb{C}}$ вообще тривиально, отображение $(\tau, z) \mapsto\left(\tau, w^{\mu}(\tau, z)\right)$ из $T(\Gamma) \times(\widehat{\mathbb{C}} \backslash \overline{\mathbb{D}})$ в $(T(\Gamma) \times \widehat{\mathbb{C}}) \backslash \overline{F(\Gamma)}$ является биголоморфизмом. В частности, дополнение к $F(\Gamma)$ заполняется голоморфным движением над всем $T(\Gamma)$. Согласно $\lambda$-лемме 2.4, это движение продолжается на границу $\partial \mathbb{D}$, и, таким образом, мы получаем, что граница $F(\Gamma)$ расслаивается в однопараметрическое семейство (графиков) голоморфных сечений над $T(\Gamma)$. (В связи с этим отметим, что расслоения Берса, за единичными исключениями $\Gamma$, не допускают глобальных голоморфных сечений, см. [34].)

Вместо круга $\mathbb{D}$ в качестве универсальной накрывающей бывает удобно использовать также верхнюю полуплоскость $\mathbb{H}$. Переход от одной модели к другой получается дробно-линейной заменой координат в $\widehat{\mathbb{C}}$, и в качестве таковой 
мы берем $h(z)=\frac{1-i}{2} \frac{z+1}{z-i}$. Отображение $h: \mathbb{D} \rightarrow \mathbb{H}$ переводит точки $-1,1, i$ на $\partial \mathbb{D}$ в точки $0,1, \infty$, что удобно при использовании нормированных движений.

Фуксовой группе $\Gamma \subset$ Aut $\mathbb{D}$ соответствует группа $\Gamma_{+}:=h \Gamma h^{-1} \subset$ Aut $\mathbb{H}$. Обозначим через $\mathscr{M}_{1}\left(\Gamma_{+}\right) \subset L^{\infty}(\mathbb{C})$ множество функций $\nu$, равных нулю вне $\mathbb{H}$ и таких, что $\|\nu\|_{\infty}<1$ и $(\nu \circ B) \overline{B^{\prime}} / B^{\prime}=\nu$ для всех $B \in \Gamma_{+}$. Тогда $\mathscr{M}_{1}\left(\Gamma_{+}\right) \ni$ $\nu \mapsto \mu:=(\nu \circ h) \overline{h^{\prime}} / h^{\prime} \in \mathscr{M}_{1}(\Gamma)$ - биголоморфное отображение, изометричное относительно нормы в $L^{\infty}(\mathbb{C})$.

Обозначим через $W^{\nu}$ единственное гомеоморфное в $\mathbb{C}$ решение уравнения Бельтрами $W_{\bar{\zeta}}=\nu W_{\zeta}$ с неподвижными точками $0,1, \infty$. Тогда $\left(W^{\nu} \circ h\right)_{z}=$ $\mu\left(W^{\nu} \circ h\right)_{z}$, т. е. $W^{\nu} \circ h-$ однолистное мероморфное решение (вне простого полюса) уравнения Бельтрами $w_{\bar{z}}=\mu w_{z}$, в общем случае не нормированное. Так как $w^{\mu}$ - решение этого же уравнения, то

$$
W^{\nu} \circ h=\frac{w^{\mu}-w^{\mu}(-1)}{w^{\mu}-w^{\mu}(i)} \frac{w^{\mu}(1)-w^{\mu}(i)}{w^{\mu}(1)-w^{\mu}(-1)} .
$$

Так как $j=-1,1, i \in \partial \mathbb{D}$, то значения $w^{\mu}(j)$ зависят только от класса Тейхмюллера $[\mu]$, и, значит, $w^{\mu_{1}}=w^{\mu_{2}}$ на $\partial \mathbb{D}$ тогда и только тогда, когда $W^{\nu_{1}}=W^{\nu_{2}}$ на $\mathbb{R}$. Таким образом, определяя $T\left(\Gamma_{+}\right)$как множество классов эквивалентности " $\nu_{1} \sim \nu_{2}$, если $W^{\nu_{1}}=W^{\nu_{2}}$ на $\mathbb{R}$ ", мы получаем биголоморфное отображение $T\left(\Gamma_{+}\right) \rightarrow T(\Gamma),[\nu] \mapsto\left[\mu:=(\nu \circ h) \overline{h^{\prime}} / h^{\prime}\right]$.

Так как $w^{\mu}$ и $W^{\nu}$ oh - однолистные решения одного уравнения Бельтрами, то $l_{\nu}:=w^{\mu} \circ h^{-1} \circ\left(W^{\nu}\right)^{-1}-$ однолистная мероморфная и, значит, дробно-линейная функция. Полагая

$$
F\left(\Gamma_{+}\right)=\left\{([\nu], \zeta): \nu \in \mathscr{M}_{1}\left(\Gamma_{+}\right), \zeta \in W^{\nu}(\mathbb{H})\right\},
$$

мы получаем, что $l_{\nu} \mid F\left(\Gamma_{+}\right)_{[\nu]} \rightarrow F(\Gamma)_{[\mu]}-$ конформные отображения. Так как $w^{\mu} \mid \partial \mathbb{D}$ и $W^{\nu} \mid \mathbb{R}$ зависят только от классов Тейхмюллера и так как представителей классов $\mu(\tau, \cdot)$, а с ними и $\nu\left(\tau_{+}, \cdot\right)$, можно выбирать голоморфно зависящими от точек пространств Тейхмюллера, то таким образом определено биголоморфное послойное отображение

$$
F\left(\Gamma_{+}\right) \ni([\nu], \zeta) \mapsto\left(\left[\mu=(\nu \circ h) \overline{h^{\prime}} / h^{\prime}\right], z=w^{\mu} \circ h^{-1} \circ\left(W^{\nu}\right)^{-1}(\zeta)\right) \in F(\Gamma) .
$$

Оно переводит орбиты действия $\Gamma_{+}$на $F\left(\Gamma_{+}\right)$в орбиты действия $\Gamma$ на $F(\Gamma)$. Таким образом, тройка $F\left(\Gamma_{+}\right) \rightarrow V\left(\Gamma_{+}\right) \rightarrow T\left(\Gamma_{+}\right)$, где $V\left(\Gamma_{+}\right)-$фактор $F\left(\Gamma_{+}\right)$ по действию $\Gamma_{+}$, биголоморфно эквивалентна $F(\Gamma) \rightarrow V(\Gamma) \rightarrow T(\Gamma)$, что позволяет отождествлять эти две модели по существу одного и того же набора расслоенных пространств.

Доказательство теоремы 2.1 во многом повторяет схему доказательства следующей теоремы Эрла-Фоулера [20], [21], которую мы здесь дополним универсальностью расслоений Берса.

ТЕОРема 3.1. Пусть $\pi: M \rightarrow B$ - голоморфное семейство гиперболических римановых поверхностей над односвязным комплексным банаховым многообразием $B$, допускающее локальную тривиализацию голоморфньми движениями, $\lambda_{0} \in B, f_{0}: \mathbb{D} \rightarrow M_{0}$ - универсальное накрытие с накрывающей группой $\Gamma$ u $f: \Omega \rightarrow M$ - соответствующая каноническая униформизация. Тогда существуют единственные голоморфные отображения $\kappa: B \rightarrow T(\Gamma), \lambda_{0} \mapsto[0]$ 
(классифицирующее отображение), и $H: M \rightarrow V(\Gamma)$ такие, что семейство $\pi: M \rightarrow B$ эквивалентно прообразу универсального семейства $V(\Gamma) \rightarrow T(\Gamma)$ относительно $\kappa$, а накрытие $f: \Omega \rightarrow M$ эквивалентно прообразу расслоения Берса $F(\Gamma) \rightarrow V(\Gamma)$ относителъно отображения $H$.

ДокАЗАТЕЛьство. Пусть $\pi: M \rightarrow B$ допускает горизонтально голоморфные тривиализации над областями $B_{\alpha}$, покрывающими $B, f_{+}:=f_{0} \circ h^{-1}$ : $\mathbb{H} \rightarrow M_{0}$ с $h$ как выше и $G_{+}-$накрывающая группа соответствующего $f_{+}$накрытия $f^{+}: \Omega^{+} \rightarrow M$. Согласно дополнению $2.1 \mathrm{k}$ теореме $2.1, G_{\lambda}^{+}=w_{\lambda} \Gamma_{+} w_{\lambda}^{-1}$ для некоторых нормированных квазиконформных гомеоморфизмов $w_{\lambda}$ плоскости $\mathbb{C}$, голоморфных в $\mathbb{C} \backslash \overline{\mathbb{H}}$, причем над каждой $B_{\alpha}$ эти $w_{\lambda}$ можно брать голоморфно зависящими от $\lambda$, сужения $w_{\lambda} \mid \mathbb{C} \backslash \mathbb{H}$ от различного выбора в одном классе коэффициентов Бельтрами не зависят и $\nu(\lambda, \cdot):=\left(w_{\lambda}\right)_{\bar{\zeta}} /\left(w_{\lambda}\right)_{\zeta} \in \mathscr{M}_{1}\left(\Gamma_{+}\right)$. В частности, $w_{\lambda}=W^{\nu(\lambda, \cdot)}$. По построению $\Omega^{+}$, имеем $W^{\nu(\lambda, \cdot)}(\mathbb{H})=\Omega_{\lambda}^{+}$.

Положим $\tau_{+}(\lambda)=[\nu(\lambda, \cdot)]$; тогда $\lambda \mapsto \tau_{+}(\lambda)-$ голоморфное отображение $B$ в $T\left(\Gamma_{+}\right)$, а сужение отображения $(\lambda, z) \mapsto\left(\tau_{+}(\lambda), z\right)$ на $\Omega^{+}$переводит эту область в $F\left(\Gamma_{+}\right)$, так как $\Omega_{\lambda}^{+}=F\left(\Gamma_{+}\right)_{[\nu(\lambda, \cdot)]}$ по построению.

Положим $\tau(\lambda)=\left[\mu(\lambda, \cdot)=(\nu(\lambda, \cdot) \circ h) \overline{h^{\prime}} / h^{\prime}\right]$; тогда $\lambda \mapsto \tau(\lambda)$ - голоморфное отображение $B$ в $T(\Gamma)$. По лемме 2.8 , существует биголоморфное отображение $g: \Omega \rightarrow \Omega^{+}$такое, что $f=f^{+} \circ g$. Поэтому

$$
\Omega \ni(\lambda, z) \mapsto\left(\tau(\lambda), w^{\mu} \circ h^{-1} \circ\left(W^{\nu}\right)^{-1} \circ g(\lambda, z)\right) \in F(\Gamma)
$$

- голоморфное послойное отображение, конформное на слоях. При этом орбиты действия $\Gamma$ на $\Omega_{\lambda}$ переходят в орбиты действия $\Gamma$ на $F(\Gamma)_{\tau(\lambda)}$, и, таким образом, это отображение индуцирует голоморфное послойное отображение $M \rightarrow V(\Gamma)$, биголоморфное на каждом слое.

Остальное очевидно.

3.2. Редукция базы. Бесконечномерные базы голоморфных семейств требуют известной осторожности при применении результатов многомерного комплексного анализа, поэтому весьма полезной может быть следующая редукция к комплексно двумерным базам.

ЛЕмма 3.1. Пусть $\pi: M \rightarrow B$ - голоморфное семейство гиперболических римановых поверхностей над односвязным комплексным банаховым многообразием. Предположим, что для всякого вложенного голоморфного двумерного шара $h: \mathbb{B}_{2} \rightarrow$ В семейство $\pi^{-1}\left(h\left(\mathbb{B}_{2}\right)\right)$ локально тривиализуется голоморфньми движениями. Тогда семейство $\pi: M \rightarrow B$ тоже локально тривиализуется голоморфными движениями и, значит, оно канонически униформизуемо.

ДокАЗАтельство. Можно считать, что $B$ - единичный шар в соответствующем банаховом пространстве и $\lambda_{0}=0$.

Фиксируем универсальное накрытие $f_{0}: \mathbb{D} \rightarrow M_{0}$. Для каждой комплексной прямой $l \ni 0$ по условию существует локальная тривиализация семейства $\pi^{-1}(B \cap l)$ голоморфными движениями. По теореме 3.1 , она определяет голоморфное отображение $\kappa_{l}: B \cap l \rightarrow T(\Gamma)$, где $\Gamma$ - накрывающая группа накрытия $f_{0}$, и согласованное с проекцией голоморфное отображение $H_{l}: \pi^{-1}(B \cap l) \rightarrow$ $V(\Gamma)$. По лемме 2.7 и теореме единственности, отображения $\kappa_{l}, H_{l}$ не зависят 
от выбора локальной тривиализации над $B \cap l$. Обозначим через $\kappa$ и $H$ отображения, соответственно равные $\kappa_{l}$ и $H_{l}$ на $B \cap l$.

Пусть П Э 0 - комплексная двумерная плоскость. По условию и тем же аргументам, существует голоморфное отображение $\kappa_{\Pi}: B \cap \Pi \rightarrow T(\Gamma)$. Тогда $\kappa_{\Pi} \mid l=\kappa_{l}$ для всех $l \ni 0$ и, значит, $\kappa_{\Pi}=\kappa \mid \Pi$, из чего следует, ввиду произвольности П, что отображение $\kappa$ голоморфно на каждом линейном диске, содержащемся в $B$. Так как $\kappa$ ограничено (вместе с $\left.T(\Gamma) \subset \mathscr{B}_{2}(\Gamma)\right)$, то это означает, что $\kappa: B \rightarrow T(\Gamma)$ - голоморфное отображение. Точно так же голоморфно и отображение $H: M \rightarrow V(\Gamma)$.

Как показано выше, $V(\Gamma)$ допускает локальную тривиализацию голоморфными движениями. Так как прообразы голоморфных движений такие же, то $M$ тоже локально тривиализуется голоморфными движениями. По теореме 2.1 с универсальным накрытием $f_{0}: \mathbb{D} \rightarrow M_{0}$, семейство $\pi: M \rightarrow B$ канонически униформизуемо. Лемма доказана.

Автор не знает, можно ли в этом общем утверждении заменить двумерные шары на голоморфные диски, однако это верно при дополнительном условии на поверхности $M_{\lambda}$.

Обозначим через $\mathbb{D}_{+}^{-}$полузамкнутый полукруг $\{z \in \mathbb{C}:|z| \leqslant 1, \operatorname{Im} z>0\}$. Мы говорим, что риманова поверхность $S$ не имеет края (или $S$ - без края), если не существует непрерывного собственного (т. е. прообраз компакта - компакт) вложения $\mathbb{D}_{+}^{-} \rightarrow S$, голоморфного внутри. Если $S$ гиперболическая, то в терминах накрывающей группы $\Gamma$ универсального накрытия $\mathbb{D} \rightarrow S$ это эквивалентно тому, что $\Gamma$ - фуксова группа первого рода, т. е. ее предельное множество совпадает с $\partial \mathbb{D}$ (= неподвижные точки преобразований из Г образуют всюду плотное подмножество $\partial \mathbb{D})$.

Напомним, что униформизацию $f: \Omega \rightarrow M$ областью $\Omega \subset B \times \mathbb{C}$ мы называем канонической (а семейство $\pi: M \rightarrow B-$ канонически униформизуемым отображением $f$ ), если

а) все слои $\Omega_{\lambda}-$ квазикруги, т. е. образы $\mathbb{D}$ при квазиконформных гомеоморфизмах плоскости, и $\Omega_{\lambda_{0}}=\mathbb{D}$,

б) граница $\Omega$ состоит из однопараметрического семейства попарно непересекающихся голоморфных графиков над $B$,

в) область $(B \times \mathbb{C}) \backslash \bar{\Omega}$ биголоморфно послойно эквивалентна $B \times(\mathbb{C} \backslash \overline{\mathbb{D}})$.

Теорема 3.2. Пусть $\pi: M \rightarrow B$ - голоморфное семейство гиперболических римановых поверхностей без края над односвязным комплексным банаховым многообразием. Предположим, что для всякого вложенного голоморфного дис$\kappa a h: \mathbb{D} \rightarrow B$ семейство $\pi^{-1}(h(\mathbb{D}))$ локально тривиализуемо голоморфными движениями. Тогда

1) для произвольной $\lambda_{0} \in B$ семейство $\pi: M \rightarrow B$ тривиализуется голоморфным движением над шаром Кобаяии $\left\{\kappa_{B}\left(\lambda, \lambda_{0}\right)<\log 2\right\}$,

2) семейство $\pi: M \rightarrow B$ канонически униформизуемо.

ДокАзАтельство. Если $B$ одномерное, то доказывать нечего, утверждения следуют из теоремы 2.1 с $D=\mathbb{D}$. Ключевым здесь является случай, когда $B$ есть единичный шар в $\mathbb{C}^{2}$, что мы сначала и предположим. 
Шаг 1. Классифицирующее отображение. Пусть Г - накрывающая группа накрытия $f_{0}: \mathbb{D} \rightarrow M_{0}$. Для каждой комплексной прямой $l \ni 0$ семейство $\pi^{-1}(l \cap B)$ удовлетворяет условиям теоремы $2.1($ с $D=\mathbb{D})$ и теоремы 3.1. Поэтому определено голоморфное отображение семейства $\pi^{-1}(l \cap B) \rightarrow l \cap B$ в $V(\Gamma) \rightarrow T(\Gamma)$, конформное в каждом слое, $\lambda \mapsto \tau_{l}(\lambda), M_{0} \rightarrow V_{[0]}=\mathbb{D} / \Gamma$. Над шаром $T_{1 / 3}(\Gamma):=\left\{\tau \in \mathscr{B}_{2}(\Gamma):\|\tau\|<1 / 3\right\}$ проекция $\mathscr{M}_{1}(\Gamma) \rightarrow T(\Gamma)$ имеет голоморфное сечение, состоящее из гармонических коэффициентов Бельтрами $\mu(\tau, z)=\left(1-|z|^{2}\right)^{2} \tau(1 / \bar{z}) / \bar{z}^{4}$ (см. п. 3.1). Прообраз этого сечения относительно отображения $\lambda \mapsto \tau_{l}(\lambda)$ обозначим через $\mu_{l}(\lambda, \cdot)$. По лемме Шварца, это сечение определено над $l \cap \frac{1}{3} B$.

По теореме 2.1, существует область $\Omega^{l} \subset(l \cap B) \times \mathbb{C}$ со свойствами а)-в) и голоморфное послойное накрытие $f^{l}: \Omega^{l} \rightarrow \pi^{-1}(l \cap B)$.

Гармонические коэффициенты определяют горизонтальную голоморфную тривиализацию $T_{1 / 3}(\Gamma)$, прообразом которой является голоморфное движение $\phi_{l}:\left(l \cap \frac{1}{3} B\right) \times M_{0} \rightarrow \pi^{-1}(l \cap B)$, причем его характеристика, соответствующая накрытию $f_{0},-$ коэффициент Бельтрами квазиконформного отображения $\phi_{l}(\lambda, \cdot): M_{0} \rightarrow M_{\lambda}-$ равна как раз $\mu_{l}(\lambda, \cdot)$.

Шаг 2. Непрерывность. Приведенная выше конструкция имеет место для любого вложенного голоморфного диска $h: \mathbb{D} \rightarrow B$, и мы покажем, что она непрерывно зависит от $h$.

Пусть $h_{j}: \mathbb{D} \rightarrow B$ сходятся к $h$ равномерно внутри $\mathbb{D}, \phi_{j}:\left(h\left(\frac{1}{3} \mathbb{D}\right)\right) \times M_{0} \rightarrow$ $\pi^{-1}\left(h\left(\frac{1}{3} \mathbb{D}\right)\right)$ - соответствующие прообразы “гармонических" голоморфных движений в $V(\Gamma)$ над $T(\Gamma)$ и $\psi_{j}:\left(\frac{1}{3} \mathbb{D}\right) \times \mathbb{D} \rightarrow \mathbb{C}-$ их канонические поднятия до нормированных голоморфных движений круга $\mathbb{D}$ (см. лемму 2.6). По лемме 2.2 , такие движения равностепенно непрерывны, и, следовательно, существует подпоследовательность $\left\{\psi_{j_{k}}\right\}$, равномерно сходящаяся на каждом $r \mathbb{D} \times \mathbb{D}, r<1 / 3$, к некоторому голоморфному движению $\psi^{1}$.

Характеристики $\mu_{j}(\zeta, \cdot)$ движений $\psi_{j}$, будучи гармоническими коэффициентами Бельтрами при фиксированных $\zeta$ и голоморфными по $\zeta$, тоже образуют нормальное семейство, и, значит, мы можем считать, что $\mu_{j_{k}} \rightarrow \mu^{1}$ равномерно внутри $\left(\frac{1}{3} \mathbb{D}\right) \times \mathbb{D}$, причем $\|\mu(\zeta, \cdot)\|_{\infty} \leqslant|\zeta|$ по лемме Шварца. Тогда $\mu^{1}-$ характеристика $\psi^{1}$, которая при каждом фиксированном $\zeta,|\zeta|<1 / 3$, является гармоническим коэффициентом Бельтрами.

Обозначим через $\psi$ движение, соответствующее, как выше, диску $h$, и пусть $\mu$ есть характеристика $\psi$. По лемме $2.7, \psi^{1}=\psi$ на $\frac{1}{3} \mathbb{D} \times \partial \mathbb{D}$, следовательно, при фиксированном $\zeta$ коэффициенты $\mu^{1}$ и $\mu$ лежат в одном классе Тейхмюллера, $\left[\mu^{1}\right]=[\mu]$. Но они оба гармонические, а гармонические коэффициенты над $T_{1 / 3}(\Gamma)$ образуют сечение. Поэтому $\mu^{1}=\mu$ и $\psi^{1}=\psi$.

Так как это верно для любой сходящейся подпоследовательности в $\left\{h_{j}\right\}$, то из этого следует, что $\psi_{j} \rightarrow \psi\left(\right.$ а тогда и $\left.\phi_{j} \rightarrow \phi\right)$, когда $h_{j} \rightarrow h$.

Шаг 3. Вариация дисков. Пусть $D_{a}$ обозначает диск $\left\{\lambda \in B: \lambda_{2}=\lambda_{1}\left(\lambda_{1}-a\right)\right\}$ и покажем, что при $|a|<1 / 5$ образы точки $(a, 0)$ в $T(\Gamma)$ относительно описанных выше классифицирующих отображений вдоль дисков $\mathbb{D} \times 0$ и $D_{a}$ совпадают. 
Положим $D^{t}=\left\{\lambda \in B: \lambda_{2}=t \lambda_{1}\left(\lambda_{1}-a\right)\right\}$, так что $D^{0}=\mathbb{D} \times 0, D^{1}=D_{a}$, и $\frac{1}{5} D^{t}=D^{t} \cap\left\{\left|\lambda_{1}\right|<1 / 5\right\}$. Пусть $\phi_{t}: \frac{1}{5} D^{t} \times M_{0} \rightarrow \pi^{-1}\left(\frac{1}{5} D^{t}\right)-$ прообразы гармонического голоморфного движения над $T_{1 / 3}(\Gamma)$, соответствующие классифицирующим отображениям $D^{t} \rightarrow T(\Gamma)$, как описано выше. Проекция круга $D^{t}$ в плоскость $\mathbb{C}_{\lambda_{1}}$ содержит круг $\left|\lambda_{1}\right|<0.6$, и потому, по лемме Шварца, образ $\frac{1}{5} D^{t}$ при классифицирующем отображении лежит в $T_{1 / 3}(\Gamma)$. По построению, $\phi_{t}(\lambda, \cdot)$ - квазиконформные отображения $M_{0}$ в $M_{\lambda}$ с гармоническими коэффициентами Бельтрами $\mu_{t}(\lambda, \cdot)$, так что при $\left|\lambda_{1}\right|<1 / 5$ классифицирующее отображение $D^{t} \rightarrow T(\Gamma)$ совпадает с $\lambda \mapsto\left[\mu_{t}(\lambda, \cdot)\right]$.

Положим $w(t, \cdot)=\phi_{t}((a, 0), \cdot)^{-1} \circ \phi_{0}((a, 0), \cdot)$. Тогда $w(t, \cdot): M_{0} \rightarrow M_{0}-$ квазиконформные отображения и $w(0, z) \equiv z$. Обозначим через $\nu(t, \cdot)$ их характеристики, соответствующие накрытию $f_{0}: \mathbb{D} \rightarrow M_{0}$, а через $W(t, \cdot)-$ квазиконформный автоморфизм $\mathbb{D}$ с дифференциалом Бельтрами $\nu(t, \cdot)$ и неподвижными точками $-1,1, i$ на $\partial \mathbb{D}$ (всякий квазиконформный автоморфизм $\mathbb{D}$ является сужением соответствующего квазиконформного автоморфизма $\mathbb{C}$ и потому непрерывно продолжается на $\overline{\mathbb{D}}$; см. [1]). Такое $W(t, \cdot)$ определено однозначно. При каждом фиксированном $t W(t, \cdot)$ переводит орбиты $\Gamma$ в орбиты $\Gamma$ (так как $\left.(\nu(t, \cdot) \circ A) \overline{A^{\prime}} / A^{\prime}=\nu(t, \cdot), A \in \Gamma\right)$, и, таким образом, $W(t, \cdot)$ является поднятием отображения $w(t, \cdot)$ относительно универсального накрытия $f_{0}: \mathbb{D} \rightarrow M_{0}$, т. е. $f_{0} \circ W(t, \cdot)=w(t, \cdot) \circ f_{0}$.

Согласно шагу $2, w:[0,1] \times M_{0} \rightarrow M_{0}$ - непрерывное отображение, и, значит, $W:[0,1] \times \mathbb{D} \rightarrow \mathbb{D}$ тоже непрерывно. Так как характеристики $\nu(t, \cdot)$ тоже непрерывны по $t$ и $|\nu(t, \cdot)|<1 / 3$, то $W$ продолжается до непрерывного отображения $W:[0,1] \times \overline{\mathbb{D}} \rightarrow \overline{\mathbb{D}}$.

Так как для всякого $A \in \Gamma$ функция $W(t, \cdot) \circ A$ является решением уравнения Бельтрами с тем же коэффициентом $\nu(t, \cdot)$, то, ввиду единственности, существует дробно-линейное отображение $A_{t} \in \mathrm{Aut} \mathbb{D}$ такое, что $A_{t}^{-1} \circ W(t, \cdot) \circ A$ имеет неподвижные точки $-1,1, i$ и, значит, совпадает с $W(t, \cdot)$, т. е. $W(t, \cdot) \circ A=$ $A_{t} \circ W(t, \cdot)$. Так как

$$
f_{0} \circ A_{t}=f_{0} \circ W(t, \cdot) \circ A \circ W(t, \cdot)^{-1}=w(t, \cdot) \circ f_{0} \circ A \circ W(t, \cdot)^{-1}=f_{0},
$$

то $A_{t} \in \Gamma$.

Так как $A_{t}$ непрерывно зависят от $t$, а группа $\Gamma$ дискретная, то $A_{t} \equiv A_{0}=A$ и, значит, $W(t, \cdot) \circ A=A \circ W(t, \cdot)$. Отображение $A$ имеет на $\partial \mathbb{D}$ одну или две неподвижные точки. Если $z_{0}$ - одна из них, то $W\left(t, z_{0}\right)=A \circ W\left(t, z_{0}\right)$, т. е. $W\left(t, z_{0}\right)$ - тоже неподвижная точка $A$ для любого $t \in[0,1]$. Так как $W(0, z) \equiv z$, то ввиду непрерывности это означает, что $W(t, z)=z$ в неподвижных точках любого преобразования $A \in \Gamma$. Так как $\Gamma$ - фуксова группа первого рода, то такие точки всюду плотны на $\partial \mathbb{D}$ и, значит, $W(t, z) \equiv z$ на $\partial \mathbb{D}$.

Положим $H=W(1, \cdot)^{-1} \circ W(0, \cdot)$ в $\mathbb{D}, H(z)=z$ в $\mathbb{C} \backslash \mathbb{D}$ и рассмотрим квазиконформное отображение $\widetilde{H}=w^{\nu(1, \cdot)} \circ H \circ\left(w^{\nu(0, \cdot)}\right)^{-1}$ плоскости $\mathbb{C}$, голоморфное в $\mathbb{C} \backslash \overline{\mathbb{D}}$. Так как $W(t, \cdot)$ и $w^{\nu(t, \cdot)}$ удовлетворяют одному уравнению Бельтрами, то функция $w^{\nu(t, \cdot)} \circ W(t, \cdot)^{-1}$ голоморфна в $\mathbb{D}$. Поэтому $\widetilde{H}=w^{\nu(1, \cdot)} \circ W(1, \cdot)^{-1} \circ W(0, \cdot) \circ\left(w^{\nu(0, \cdot)}\right)^{-1}$ голоморфно и в $\mathbb{D}$. Так как отображение $\widetilde{H}$ непрерывное, то оно голоморфное в $\mathbb{C}$ и, значит, аффинное, а так как $\widetilde{H}(z)=z+o(1)$ в окрестности $\infty$, то $\widetilde{H}(z) \equiv z$. Следовательно, $w^{\nu(1, \cdot)}=w^{\nu(0, \cdot)}$ 
в $\mathbb{C} \backslash \mathbb{D}$, в частности на $\partial \mathbb{D}$. По определению классов Тейхмюллера, $[\nu(1, \cdot)]=$ $[\nu(0, \cdot)]$.

Шаг 4. Голоморфность классифицирующего отображения. Вместо диска $B \cap\left\{\lambda_{2}=0\right\}$ в предыдущем шаге можно было рассматривать любой диск $B \cap\left\{\lambda_{2}=c \lambda_{1}\right\}$ с малым $|c|$ и с теми же дисками $D_{a}$. Соответственно меняя гомотопию $D^{t}$ так, что все $D^{t}$ содержат точку $D_{a} \cap\left\{\lambda_{2}=c \lambda_{1}\right\}$, мы получаем, как выше, что образ этой точки при классифицирующих отображениях дисков $B \cap\left\{\lambda_{2}=c \lambda_{1}\right\}$ и $D_{a}$ в $T(\Gamma)$ совпадают.

Таким образом, классифицирующее отображение $\kappa: B \rightarrow T(\Gamma)$ из шага 1 голоморфно не только вдоль дисков $B \cap\left\{\lambda_{2}=c \lambda_{1}\right\}$, но - вблизи $(0,0)-$ и вдоль дисков $D_{a}$. Так как оба семейства голоморфны (по параметрам $c$ и $f$ ), то по теореме Гартогса $\kappa$ голоморфно в секторе $\left\{\lambda:\left|\lambda_{1}\right|<1 / 5,\left|\lambda_{2}\right|<\delta\left|\lambda_{1}\right|\right\}$ с некоторым $\delta>0$. Унитарным преобразованием $\mathbb{C}^{2}$ любую прямую $l \ni 0$ можно перевести в $\left\{\lambda_{2}=0\right\}$, поэтому $\kappa$ голоморфно в проколотом шаре $\frac{1}{5} B \backslash 0$. Так как $T(\Gamma)$ содержится в единичном шаре банахова пространства $\mathscr{B}_{2}(\Gamma)$, то особенность в 0 устранима и, значит, $\kappa$ голоморфно в $\frac{1}{5} B$. Так как оно голоморфно и на каждом диске $l \cap B \ni 0$, то по лемме Гартогса $\kappa$ голоморфно в $B$.

Шаг 5. Общий случай. По доказанному и лемме 3.1 , семейство $\pi: B \rightarrow M$ локально тривиализуется голоморфными движениями. Остальное очевидно следует из теоремы 2.1 (с $D=\mathbb{D}$ ) и теоремы 3.1: метрика Кобаяши не увеличивается при голоморфных отображениях, поэтому образ шара Кобаяши $B_{\log 2}=\left\{\kappa_{B}\left(\lambda, \lambda_{0}\right)<\log 2\right\}$ при классифицирующем отображении $B$ в единичный шар пространства $\mathscr{B}_{2}(\Gamma)$, содержащий $T(\Gamma)$, лежит в $T_{1 / 3}(\Gamma)$, так как расстояние Кобаяши до 0 в этом шаре равно $\log ((1+\|\tau\|) /(1-\|\tau\|))$ и $\|\tau\|<1 / 3$, если это выражение меньше $\log 2$. Теорема доказана.

Ключевая часть доказательства, шаг 3 , взята из доказательства леммы II в работе Берса и Ройдена [12].

3.3. Теорема Берса-Ройдена. Гармонические коэффициенты Бельтрами существенно используются также в доказательстве теоремы Берса-Ройдена [12] о продолжении голоморфных движений.

Несколько слов о терминологии. Пусть $\Gamma$ - накрывающая группа универсального накрытия $\mathbb{D} \rightarrow D$ плоской области $D \subset \mathbb{C}_{z}$ и $\mu \in \mathscr{M}_{1}(\Gamma)$ - коэффициент Бельтрами в $\mathbb{D} \subset \mathbb{C}_{\zeta}$. Тогда дифференциал Бельтрами $\mu d \bar{\zeta} / d \zeta$ инвариантен относительно Г и, значит, является поднятием однозначно определенного дифференциала Бельтрами $\nu d \bar{z} / d z$ в $D$. Метрика Пуанкаре $2|d \zeta| /\left(1-|\zeta|^{2}\right)$ в $\mathbb{D}$ является поднятием в $\mathbb{D}$ гиперболической метрики (= метрики Кобаяши) $\rho_{D}|d z|$ на $D$. Если коэффициент $\mu$ гармонический, то функция $\mu /\left(1-|\zeta|^{2}\right)$ антиголоморфна в $\mathbb{D}$ по определению и, значит, функция $\rho_{D}^{2} \nu$ антиголоморфна в $D$. Поэтому гармоническими коэфбиииентами Бельтрами в области $D \subset \mathbb{C}$ называются функции вида $\bar{h} / \rho_{D}^{2}$, где $h$ голоморфна в $D$ и $\left\|h / \rho_{D}^{2}\right\|_{\infty}<1$.

Теорема 3.3. Пусть $\phi: B \times E \rightarrow \mathbb{C}$ - нормированное голоморфное движение множества $E \subset \mathbb{C}$ над односвязным комплексным банаховым многообразием $\left(B, \lambda_{0}\right)$. Тогда существует голоморфное движение $\tilde{\phi}: B_{\log 2} \times \mathbb{C} \rightarrow \mathbb{C}$ 
maкое, что $\tilde{\phi}=\phi н а B_{\log 2} \times E$. Движение $\tilde{\phi}$ определяется однозначно дополнительным условием, что его характеристика при каждом фиксированном $\lambda \in B_{\log 2}$ является гармоническим коэфбициентом Бельтрами в каждой связной компоненте множества $\mathbb{C} \backslash \bar{E}$. При этом, если $D$ - такая компонента и в области $\left\{(\lambda, w): \lambda \in B_{\log 2}, w=\tilde{\phi}(\lambda, z), z \in D\right\}$ действует группа $G$ биголоморфных послойных автоморфизмов $(\lambda, w) \mapsto\left(\lambda, g_{\lambda}(w)\right)$, то это единственное движение $\tilde{\phi}$ инвариантно относительно $G$, точнее, $g_{\lambda} \circ \tilde{\phi}(\lambda, \cdot)=\tilde{\phi}(\lambda) \circ g_{\lambda_{0}} \partial л я$ всех $g_{\lambda} \in G_{\lambda}, \lambda \in B_{\log 2}, z \in D$.

Здесь $B_{\log 2}$ есть шар Кобаяши $\left\{\lambda \in B: \kappa_{B}\left(\lambda, \lambda_{0}\right)<\log 2\right\}$; если $B-$ шар $\{\|\lambda\|<R\}$ в банаховом пространстве, то $B_{\log 2}$ есть шар $\{\|\lambda\|<R / 3\}$.

ДокАзАТЕльство. Приводим сокращенное доказательство, отсылая за подробностями к [12].

Сначала разберем случай конечного множества $E=\left\{0,1, z_{1}, \ldots, z_{n}\right\}$ (если $E=\{0,1\}$, то доказывать нечего). Голоморфное движение $\phi$ определяет тогда голоморфное отображение $\Phi: B \rightarrow \mathbb{C}^{n}, \Phi(\lambda)=\left(\phi\left(\lambda, z_{1}\right), \ldots, \phi\left(\lambda, z_{n}\right)\right)$. Так как значения функций $\phi\left(\cdot, z_{j}\right)$ при различных $z_{j}$ различные, то значения $\Phi$ лежат в области

$$
M_{n}=(\mathbb{C} \backslash\{0,1\})^{n} \backslash \bigcup_{j \neq k}\left\{z_{j}=z_{k}\right\} .
$$

Пусть $\Gamma$ - накрывающая группа универсального накрытия $\mathbb{D} \rightarrow \mathbb{C} \backslash E$. Тогда, как легко понять, пространство Тейхмюллера $T(\Gamma)$ биголоморфно эквивалентно факторпространству функций $\mu \in L^{\infty}(\mathbb{C})$ по отношению эквивалентности " $\mu_{1} \equiv \mu_{2}$, если $w^{\mu_{1}}=w^{\mu_{2}}$ во всех точках множества $E$ ". Из этого несложно вывести, что $T(\Gamma)$ является универсальным накрывающим многообразием области $\mathbb{C} \backslash E$, причем универсальное накрытие определяется как $[\mu] \mapsto\left(w^{\mu}\left(z_{1}\right), \ldots, w^{\mu}\left(z_{n}\right)\right)$; это "хорошо известно" (см. [12] и [47]).

Так как $B$ односвязно, то отображение $\Phi: B \rightarrow M_{n}$ поднимается до голоморфного отображения $\tilde{\Phi}: B \rightarrow T(\Gamma)$, однозначно определяемого условием $\lambda_{0} \mapsto[0]$. По лемме Шварца для метрики Кобаяши (п. 2.1), $\tilde{\Phi}\left(B_{\log 2}\right)$ содержится в $T_{1 / 3}(\Gamma)=\left\{\tau \in \mathscr{B}_{2}(\Gamma):\|\tau\|<1 / 3\right\}$.

Над $T_{1 / 3}(\Gamma)$ есть голоморфное сечение $\mu(\tau, \cdot)$ проекции $\mu \mapsto[\mu]$, состоящее из гармонических коэффициентов Бельтрами в $\mathbb{C} \backslash E$ (см. выше) при $\tau \in T_{1 / 3}(\Gamma)$. Положим $\tilde{\phi}(\lambda, \cdot)=w^{\mu(\tilde{\Phi}(\lambda), \cdot)}$. Тогда $\tilde{\phi}: B_{\log 2} \times \mathbb{C} \rightarrow \mathbb{C}-$ голоморфное движение $\mathbb{C}$ над $\left(B_{\log 2}, \lambda_{0}\right)$. Так как $\tilde{\Phi}(\lambda) \mapsto\left(\tilde{\phi}\left(\lambda, z_{1}\right), \ldots, \tilde{\phi}\left(\lambda, z_{n}\right)\right)$ при универсальном накрытии $T(\Gamma) \rightarrow M_{n}$ (см. выше) и $\tilde{\Phi}$ есть поднятие $\Phi$, то $\left(\tilde{\phi}\left(\lambda, z_{1}\right), \ldots, \tilde{\phi}\left(\lambda, z_{n}\right)\right)=$ $\left(\phi\left(\lambda, z_{1}\right), \ldots, \phi\left(\lambda, z_{n}\right)\right)$, т. е. $\tilde{\phi}$ - искомое продолжение $\phi$.

Пусть теперь $E \subset \mathbb{C}$ произвольно и $\{0,1\} \subset E_{1} \subset E_{2} \subset \cdots$ - конечные подмножества $E$ такие, что $\bigcup E_{j}$ всюду плотно в $E$. Пусть $\tilde{\phi}_{n}$ - описанные выше продолжения голоморфных движений $\phi \mid B \times E_{n} \rightarrow \mathbb{C}$. Предположим сначала, что $B$ конечномерно. Пусть $\left\{w_{i}\right\}$ - последовательность, всюду плотная в $\mathbb{C} \backslash E$. Согласно следствию 2.2 , из $\left\{\tilde{\phi}_{n}\right\}$ можно выделить (диагональным процессом) подпоследовательность $\left\{\tilde{\phi}_{n_{k}}\right\}$ такую, что голоморфные в $B_{\log 2}$ функции $\tilde{\phi}_{n_{k}}\left(\lambda, w_{i}\right)$ сходятся равномерно внутри $B$ к соответствующим голоморфным функциям $\tilde{\phi}^{i}$. Из неравенств Харнака легко следует (см., например, [18]), что в каждой точке $\lambda$ значения функций $\tilde{\phi}^{i}$ и $\tilde{\phi}^{j}$ различны, если $w_{i} \neq w_{j}$. 
Таким образом, полагая $\tilde{\phi}\left(\lambda, w_{i}\right)=\tilde{\phi}^{i}(\lambda)$, мы получаем голоморфное движение над $B_{\log 2}$ множества $\left(\bigcup E_{n}\right) \cup\left\{w_{i}\right\}$, всюду плотного в $\mathbb{C}$. По $\lambda$-лемме 2.4 , оно однозначно продолжается до искомого голоморфного движения всей плоскости $\mathbb{C}$.

Если $B$ бесконечномерно, то это рассуждение не проходит (скажем, в таком банаховом шаре даже линейные функции не образуют нормальное семейство). Здесь надо вернуться к гармоническим коэффициентам Бельтрами и использовать единственность $\tilde{\phi}$ с такой характеристикой, доказанную в [12; с. 275-278] для $B=\mathbb{D}$.

Пусть $\tilde{\phi}_{n}$ определены как выше, $D$ - связная компонента $\mathbb{C} \backslash \bar{E}$ и $\rho_{n}-$ гиперболическая метрика в $\mathbb{C} \backslash E_{n}$, а $\rho_{D}-$ в $D$. Тогда $\left.\rho_{n}\right|_{D} \rightarrow \rho_{D}$ при $n \rightarrow \infty$ (это несложно [12; с. 274-275]). Так как характеристики $\mu_{n}$ движений $\tilde{\phi}_{n}$ при каждом фиксированном $\lambda^{\prime} \in B_{\log 2}$ являются гармоническими коэффициентами Бельтрами, то из $\left\{\tilde{\phi}_{n}\left(\lambda^{\prime}, \cdot\right)\right\}$ выделяется подпоследовательность, сходящаяся к некоторому квазиконформному отображению $\psi_{\lambda^{\prime}}: \mathbb{C} \rightarrow \mathbb{C}$, причем сужение $\psi_{\lambda^{\prime}}$ на каждую связную компоненту $\mathbb{C} \backslash \bar{E}$ имеет гармонический коэффициент Бельтрами. Пусть $h: \mathbb{D} \rightarrow B, h(0)=\lambda_{0}$, - голоморфный диск, содержащий $\lambda^{\prime}$. Согласно указанной выше единственности, предельная функция $\psi_{\lambda^{\prime}}$ не зависит от выбора подпоследовательности в $\left\{\tilde{\phi}_{n}\left(\lambda^{\prime}, \cdot\right)\right\}$, т. е. $\left\{\tilde{\phi}_{n}\left(\lambda^{\prime}, \cdot\right)\right\} \rightarrow \psi_{\lambda^{\prime}}$ равномерно на компактных подмножествах $\mathbb{C}$. Таким образом, если $\mathbb{B} \subset B-$ координатный шар в $B$, содержащий $\lambda_{0}$, то последовательность $\left\{\tilde{\phi}_{n}\right\}$ сходится в $\mathbb{B} \times \mathbb{C}$. Так как $\tilde{\phi}_{n}(\cdot, z)$ равномерно ограничены на шарах Кобаяши многообразия $B_{\log 2}$ (лемма 2.2), то предельная функция $\tilde{\phi}$ голоморфна по $\lambda$ и, как выше, $\tilde{\phi}: \mathbb{B} \times \mathbb{C} \rightarrow \mathbb{C}$ есть голоморфное движение, продолжающее $\phi$ над $\mathbb{B}$.

Покрывая произвольный путь $[0,1] \rightarrow B_{\log 2}$ конечным числом координатных шаров, мы получаем, что последовательность $\left\{\tilde{\phi}_{n}(\lambda, \cdot)\right\}$ сходится при любом фиксированном $\lambda \in B_{\log 2}$ и предельное голоморфное движение - искомое.

Концовка об инвариантности очевидно следует из единственности, поскольку конформные автоморфизмы слоев сохраняют гиперболическую метрику и, значит, переводят гармонические коэффициенты Бельтрами в такие же.

ЗАмечаниЕ 3.1. Всякое голоморфное движение $\phi: U \times \mathbb{C} \rightarrow \mathbb{C}$ всей плоскости определяет горизонтально голоморфную тривиализацию $(\lambda, z) \mapsto(\lambda, \phi(\lambda, z))$ семейства $U \times \mathbb{C} \rightarrow U$ : так как образ $\mathbb{C}$ относительно любого квазиконформного отображения $\phi(\lambda, \cdot)$ односвязен и не существует квазиконформного отображения $\mathbb{C}$ на $\mathbb{D}$, то, по теореме Римана, $\phi(\lambda, \mathbb{C})=\mathbb{C}$ для каждого $\lambda \in U$.

Теорема 3.3 доказана Берсом и Ройденом для $B=\mathbb{D}$ (см. также [11]). Сугава [58] отметил, не вдаваясь в детали, что их доказательство проходит и для шаров в банаховых пространствах, но, как видим, оно работает и в самом общем случае.

Для $B=\mathbb{D}$ теорема о продолжении движений существенно усилена Слодковским, который доказал (см. [56], [57]), что всякое голоморфное движение $\phi: \mathbb{D} \times E \rightarrow \mathbb{C}$ над $(\mathbb{D}, 0)$ продолжается до голоморфного движения $\tilde{\phi}$ всей плоскости $\mathbb{C}$ над всем $(\mathbb{D}, 0)$, причем это продолэение можно выбирать инвариантным относительно действий послойных биголоморфизмов связных компонент дополнения к пространству движения $\phi$. 
Есть уже несколько различных доказательств этой важной для приложений теоремы (см. [5], [27]; простое доказательство существования $\tilde{\phi}$ см. в [15]), и мы здесь не будем повторяться. Теорема Слодковского не распространяется на движения уже над двумерными односвязными базами, поэтому в многомерных задачах с локальными движениями удобнее пользоваться теоремой Берса-Ройдена.

СлЕДСтвиЕ 3.1. Пусть $\pi: M \rightarrow B$ - голоморфное семейство гиперболических римановых поверхностей без края над комплексным банаховым многообразием. Предположим, что есть область $\Omega \subset B \times \mathbb{C}$ с ограниченными односвязными слоями такими, что $\Omega_{\lambda}=\operatorname{int} \overline{\Omega_{\lambda}}$ для всех $\lambda \in B$, и есть голоморфное послойное накрытие $f: \Omega \rightarrow M$. Тогда семейство $\pi: M \rightarrow B$ локально тривиализуется голоморфными движениями.

ДоказАтельство. Пусть $s: U \rightarrow M-$ голоморфное сечение $\pi$ над односвязной областью $U \subset B, \lambda_{0} \in U$ и $G$ - накрывающая группа накрытия $f$. Тогда $f^{-1}(s)$ состоит из счетного числа попарно непересекающихся голоморфных графиков над $U$. Так как $M_{\lambda}$ не имеют края и $\Omega_{\lambda}=\operatorname{int} \overline{\Omega_{\lambda}}$, то множество предельных точек орбиты $f^{-1}(s(\lambda))$ группы $G_{\lambda}$ совпадает с $\partial \Omega_{\lambda}$ (см. конформное отображение $\Omega_{\lambda}$ на $\left.\mathbb{D}\right)$.

Графики из $f^{-1}(s)$ образуют голоморфное движение множества $f^{-1}\left(s\left(\lambda_{0}\right)\right)$ над $\left(U, \lambda_{0}\right)$. По $\lambda$-лемме 2.4 , оно продолжается до голоморфного движения замыкания этого множества, в частности до голоморфного движения $\phi$ границы $\partial \Omega_{\lambda_{0}}$, включающего все графики, предельные для $f^{-1}(s)$. По теореме Берса-Ройдена, существует голоморфное движение $\tilde{\phi}: U_{\log 2} \times \mathbb{C} \rightarrow \mathbb{C}$, продолжающее $\phi$ и инвариантное относительно $G$ в $\Omega$. Поэтому в $\pi^{-1}\left(U_{\log 2}\right)$ есть тривиализующее голоморфное движение (включающее $s \mid U_{\log 2}$ ). Так как голоморфные сечения проекции $\pi$ локально существуют всюду, то утверждение доказано.

В некотором смысле это утверждение показывает необходимость локальной тривиализуемости семейства $\pi: M \rightarrow B$ голоморфными движениями для существования его более-менее хорошей униформизации и служит еще одной мотивацией естественности этого свойства в задачах униформизации семейств.

Вместе с тем, само существование локальной горизонтально голоморфной тривиализации представляется на первый взгляд столь же туманным, как сама проблема униформизации (см. [20; с. 251]).

В следующем разделе мы подготовим технику, позволяющую строить тривиализации голоморфными движениями при некоторых условиях на семейства $\pi: M \rightarrow B$ типа псевдовыпуклости, обычных для многомерного комплексного анализа.

\section{4. Почти голоморфные движения}

4.1. Квазилинейные уравнения Бельтрами. Почти комплексная структура на гладком банаховом многообразии $M$ - это непрерывный послойный оператор $J$ в касательном расслоении $T M, \mathbb{R}$-линейный в слоях и такой, что $J^{2}=-I$, где $I$ - тождественное отображение. 
Комплексифицированное кокасательное пространство $\mathbb{C} T_{p}^{*} M$ в точке $p \in$ $M$ - это пространство всех комплекснозначных $\mathbb{R}$-линейных функций на $T_{p} M$. Пространства $\mathbb{C} T_{p}^{*} M$ образуют комплексифицированное кокасательное расслоение $\mathbb{C} T^{*} M$. Если $J$ - оператор почти комплексной структуры на $M$ и $\alpha \in$ $\mathbb{C} T_{p}^{*} M$, то, полагая $\left(J^{*} \alpha\right)(v):=\alpha(J v)$ для любых $v \in T_{p} M$, мы получаем аналогичный, но уже $\mathbb{C}$-линейный в слоях оператор $J^{*}$ с $\left(J^{*}\right)^{2}=-I$. По существу $J$ и $J^{*}$ - это один и тот же объект (почти комплексная структура) на многообразии $M$, поэтому далее оператор $J^{*}$ тоже называется почти комплексной структурой на $M$ и обозначается той же буквой $J$.

Структура $J$ в $\mathbb{C} T^{*} M$ определяет разложение этого расслоения в прямую сумму дифференциалов (1-форм) типа $(1,0)_{J}$ ("голоморфных") и типа $(0,1)_{J}$ (“антиголоморфных"): $\alpha$ имеет тип $(1,0)_{J}$, если $J \alpha=i \alpha$, и тип $(0,1)_{J}$, если $J \alpha=-i \alpha$. Таким образом, $(1,0)_{J}$-компонента общей 1 -формы $\alpha$ равна $(\alpha-$ $i J \alpha) / 2$, а компонента типа $(0,1)_{J}$ равна $(\alpha+i J \alpha) / 2$.

Функция $f$ класса $C^{1}$ в области $U$ называется $J$-голоморфной, если ее дифференциал в каждой точке $U$ имеет тип $(1,0)_{J}$, т. е. выполняется условие КошиРимана $J d f=i d f$.

Нас интересуют почти комплексные структуры $J$ в областях $\Omega \in \mathbb{C}_{\lambda, w}^{2}$, относительно которых функция $\lambda$ (проекция в $\mathbb{C}_{\lambda}$ ) голоморфна, а сужение $J$ на слои $\Omega_{\lambda}$ сохраняет ориентацию. Такие структуры мы называем далее согласованными (с проекцией в $\left.\mathbb{C}_{\lambda}\right)$.

В каждой точке $\Omega$ формы типа $(1,0)_{J}$ образуют комплексное двумерное про-

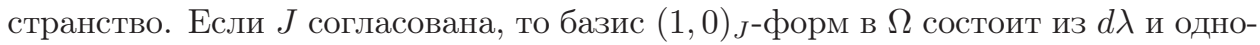
значно определенной формы $\alpha=d w-\mu d \bar{w}-a d \bar{\lambda}$ с коэффициентами, непрерывными, гладкими и т. п., как сам оператор $J$. Так как $J$ сохраняет ориентацию в слоях $\Omega_{\lambda}$, то $|\mu|<1$. С другой стороны, любая такая форма $\alpha$ вместе $\mathrm{c} d \lambda$ определяет в $\Omega$ согласованную почти комплексную структуру $J$, относительно которой $\alpha$ имеет тип $(1,0)_{J}$, так как любая 1 -форма в $\Omega$ поточечно есть $\mathbb{C}$-линейная комбинация $d \lambda, \alpha$ и $d \bar{\lambda}, \bar{\alpha}$. Поэтому всюду далее эта $\alpha$ называется определяющей формой структуры $J$.

Принадлежащий $\Omega$ график $S: w=\phi(\lambda)$ функции $\phi$ класса $C^{1}(U)$ в некоторой области $U \subset \mathbb{C}_{\lambda}$ является $J$-голоморфным, если сужения на него форм $d \lambda$ и $\alpha$ линейно зависимы в каждой точке, т. е. $(d w-\mu d \bar{w}-a d \bar{\lambda}) \wedge d \lambda \equiv 0$ в $U$. Это условие переписывается в виде квазилинейного уравнения Бельтрами

$$
\phi_{\bar{\lambda}}-\mu(\cdot, \phi) \overline{\phi_{\lambda}}=a(\cdot, \phi),
$$

которое мы исследуем в этом пункте. Нам понадобятся такие уравнения с кусочно непрерывными по $\lambda$ коэффициентами $\mu, \phi$, поэтому наряду с функциями класса $C^{1}$ и их графиками мы будем использовать также кусочно $C^{1}$-функции, а уравнение (8) удобнее решать вообще в классах Соболева $W^{1, p}(U)$ (с подходящими $p>2$ ), состоящих из непрерывных функций, первые частные производные которых в смысле распределений принадлежат $L^{p}(U)$. Графики таких решений будем называть $J$-голоморфными. Они составляют сравнительно простой подкласс общих псевдоголоморфных кривых, введенных Громовым [31].

Псевдоголоморфные кривые обладают многими свойствами голоморфных кривых, и на эту тему есть довольно богатая литература (см., например, [6]). Однако нам понадобятся лишь простейшие свойства, которые мы приведем с доказательствами для полноты изложения. 
Доопределяя $\mu$ и $а$ нулем вне $\Omega$ (т. е. полагая там $J$ равной $J_{\mathrm{st}}$, стандартной структуре в $\left.\mathbb{C}^{2}\right)$, мы можем решать уравнение $(8)$ во всей плоскости $\mathbb{C}$, не обращая внимания на форму конкретной области $\Omega$.

Обозначим (следуя И.Н. Векуа) через П плоский сингулярный оператор Гильберта

$$
(\Pi h)(\lambda)=-\frac{1}{\pi} \int_{\mathbb{C}} \frac{h(\zeta) d S_{\zeta}}{(\zeta-\lambda)^{2}},
$$

который действует из $L^{p}(\mathbb{C})$ в $L^{p}(\mathbb{C})$ при любом $p>1$, причем его норма $\|\Pi\|_{p}$ в $L^{p}$ является непрерывной функцией от $p$ и $\|\Pi\|_{2}=1$. С плоским оператором Коши

$$
(T h)(\lambda)=-\frac{1}{\pi} \int_{\mathbb{C}} \frac{h(\zeta) d S_{\zeta}}{\zeta-\lambda}
$$

оператор П связан замечательным соотношением $(T h)_{\lambda}=\Pi h$ в смысле распределений (см. [60; гл. 1]).

ЛЕмма 4.1. Пусть функиии $\mu$ и а определенъ и измеримы в $\mathbb{C}_{w}$ при почти каждом фиксированном $\lambda$, причем $\mu$ имеет компактный носитель, $\|\mu\|_{\infty}<1$ и бункиця $a(\lambda, w)\left(1+|\lambda|^{2}\right)$ равномерно ограничена. Пусть $p>2$ таково, что $\|\Pi\|_{p}\|\mu\|_{\infty}<1$. Тогда для любой $\lambda_{0} \in \widehat{\mathbb{C}}$ и любого $z \in \mathbb{C}$ уравнение (8) имеет непрерывное в $\widehat{\mathbb{C}}$ решение $\phi$ такое, что $\phi_{\bar{\lambda}} \in L^{p}(\mathbb{C}) u \phi\left(\lambda_{0}\right)=z$. При этом $|\phi-z| u\left\|\phi_{\bar{\lambda}}\right\|_{p}$ не превосходят $C\|a\|_{\infty}$ с константой $C$, зависящей только от $p u\|\mu\|_{\infty}$.

(Здесь и далее $\|\cdot\|_{p}$ означает норму в соответствующем $L^{p}$.)

ДокАЗАТЕЛЬСтво. Положим

$$
\left(T_{0} h\right)(\lambda)=-\frac{1}{\pi} \int_{\mathbb{C}} h(\zeta)\left(\frac{1}{\zeta-\lambda}-\frac{1}{\zeta-\lambda_{0}}\right) d S .
$$

Если уравнение $(8)$ имеет решение $\phi \in W^{1, p}(\mathbb{C})$, то это решение представляется в виде $\phi=z+T_{0} \phi_{\bar{\lambda}}$. Для таких $\phi(8)$ переписывается в виде

$$
(1-\mu(\cdot, \phi) Q \Pi) \phi_{\bar{\lambda}}=a(\cdot, \phi),
$$

где $Q$ - оператор комплексного сопряжения. Если $p>2$ таково, что выполнено условие $\|\Pi\|_{p}\|\mu\|_{\infty}<1$, то оператор $1-\mu(\cdot, \phi) Q \Pi$ в $L^{p}(\mathbb{C})$ обратим (при каждой фиксированной $\phi),(1-\mu(\cdot, \phi) Q \Pi)^{-1}=1+\sum_{1}^{\infty}(\mu(\cdot, \phi) Q \Pi)^{n}$. Фиксируем такое $p$; тогда (8) эквивалентно уравнению $\phi_{\bar{\lambda}}=(1-\mu(\cdot, \phi) Q \Pi)^{-1} a(\cdot, \phi)$.

Так как $\left(T_{0} h\right)_{\bar{\lambda}}=h$, то при фиксированном $z \in \mathbb{C}$ уравнение (8) с указанным "начальным условием" эквивалентно интегральному уравнению $\phi=K_{0} \phi$, где

$$
K_{0} \phi=z+T_{0}(1-\mu(\cdot, \phi) Q \Pi)^{-1} a(\cdot, \phi)
$$

в классе функций $\phi \in C(\widehat{\mathbb{C}})$ таких, что $\phi_{\bar{\lambda}} \in L^{p}$. Это банахово пространство с нормой $\|\phi\|=\left|\phi\left(\lambda_{0}\right)\right|+\left\|\phi_{\bar{\lambda}}\right\|_{p}$. Так как $\mu$ имеет компактный носитель и функция $a(\lambda, w)\left(1+|\lambda|^{2}\right)$ равномерно ограничена, то оператор $K_{0}$ имеет ограниченный образ, $\left\|K_{0} \phi\right\| \leqslant R$ с константой $R>0$, не зависящей от $\phi$. Так как оператор $T$ переводит функции $h \in L^{p}(\mathbb{C})$, для которых $|h(\lambda)|\left(1+|\lambda|^{2}\right)$ ограничено, в функции, удовлетворяющие условию Гёльдера и стремящиеся к нулю при $\lambda \rightarrow \infty$ 
(см. [60; теорема 1.24]), а $T_{0} h=T h-T h\left(\lambda_{0}\right)$, то $K_{0}-$ компактный оператор. По теореме Шаудера о неподвижной точке, в указанном пространстве существует функция $\phi$ такая, что $\phi=K_{0} \phi$, а это и есть решение $\phi$ уравнения (8) c $\phi\left(\lambda_{0}\right)=z$.

Указанные оценки очевидным образом следуют из представления $\phi=z+$ $T_{0}(1-\mu(\cdot, \phi) Q \Pi)^{-1} a(\cdot, \phi)$. Лемма доказана.

СлЕДСТВИЕ 4.1. Если $\phi$ - решение уравнения (8) из леммы 4.1, по $\phi_{\bar{\lambda}} \in$ $L^{q}(\mathbb{C})$ для всех $q$ таких, что $\|\Pi\|_{q}\|\mu\|_{\infty}<1$ (окрестность $q=2$ в $\mathbb{R}$ ).

ДоказАТЕльство. Действительно, по доказанному, $\phi=z+T_{0}(1-\mu Q \Pi)^{-1} C$, где $C \in L^{s}(\mathbb{C})$ для всех $s>1$ по условию леммы 4.1. Оператор П действует в каждом $L^{s}(\mathbb{C})$ и при указанных $q$ оператор $1-\mu Q \Pi$ в $L^{q}$ обратим.

Для единственности решения нужны дополнительные условия.

Лемма 4.2. Пусть функиии $\mu$, а и р такие, как в условии леммы 4.1, причем $\mu(\lambda, \cdot)$ u $\left(1+|\lambda|^{2}\right) a(\lambda, \cdot)$ для почти всех $\lambda \in \mathbb{C}$ удовлетворяют условию Липшица по ш с константой, не зависящей от $\lambda$. Пусть $\phi_{1}, \phi_{2}$ - непрерьвнъе в $\widehat{\mathbb{C}}$ решения уравнения (8) такие, что $\left(\phi_{j}\right)_{\bar{\lambda}} \in L^{p}(\mathbb{C})$ u $\phi_{1}\left(\lambda_{0}\right)=\phi_{2}\left(\lambda_{0}\right)$ в некоторой точке $\lambda_{0} \in \widehat{\mathbb{C}}$. Тогда $\phi_{1} \equiv \phi_{2}$.

ДоказАТельство. Положим $f=\phi_{1}-\phi_{2}$. Тогда $f_{\bar{\lambda}} \in L^{p}(\mathbb{C})$. Из указанных условий Липшица получаем, что

$$
f_{\bar{\lambda}}-\mu_{1} \overline{f_{\lambda}}=\left(\mu\left(\cdot, \phi_{1}\right)-\mu\left(\cdot, \phi_{2}\right)\right) \overline{\left(\phi_{2}\right)_{\lambda}}-\left(a\left(\cdot, \phi_{1}\right)-a\left(\cdot, \phi_{2}\right)\right)=: C f
$$

с $\mu_{1}:=\mu\left(\cdot, \phi_{1}\right)$, причем функция $C(\lambda)\left(1+|\lambda|^{2}\right)$ равномерно ограничена вне проекции носителя $\mu$ и, значит, $C \in L^{p}(\mathbb{C})$.

Положим $\mu_{2}=\mu_{1} \bar{f} / f$ там, где $f \neq 0$, и $\mu_{2}=0$, где $f=0$, и пусть $c=$ $T\left(1-\mu_{2} Q \Pi\right)^{-1} C$. Тогда $c_{\bar{\lambda}}-\mu_{2} \overline{c_{\lambda}}=C$ там, где $f \neq 0$, и значит, $\left(c_{\bar{\lambda}}-\mu_{2} \overline{c_{\lambda}}\right) f=C f$ в $L^{p}(\mathbb{C})$. Так как $C \in L^{p}(\mathbb{C})$, то $\left(1-\mu_{2} Q \Pi\right)^{-1} C=C+B$, где $B-$ финитная функция из $L^{p}(\mathbb{C})$, и поэтому функция $c$ непрерывна в $\widehat{\mathbb{C}}$ (см. [60; гл. 1]).

Для обобщенных производных по $\lambda, \bar{\lambda}$ функции $f e^{-c}$ применимо правило Лейбница: если $f_{\varepsilon}, c_{\varepsilon}-$ стандартные усреднения, то $c_{\varepsilon} \rightarrow c, f_{\varepsilon} e^{-c_{\varepsilon}} \rightarrow f e^{-c}$ равномерно и, значит,

$$
\left(f e^{-c}\right)_{\bar{\lambda}}-e^{\bar{c}-c} \mu_{1} \overline{\left(f e^{-c}\right)_{\lambda}}=e^{-c}\left(f_{\bar{\lambda}}-\mu_{1} \overline{f_{\lambda}}-\left(c_{\bar{\lambda}}-\mu_{2} \overline{c_{\lambda}}\right) f\right)=0 .
$$

Положим $h=f e^{-c}$. Функция $h$ непрерывна в $\widehat{\mathbb{C}}$, равна нулю при $\lambda=\lambda_{0}$ и $h_{\bar{\lambda}} \in L^{p}(\mathbb{C})$, поэтому $h=T_{0} h_{\bar{\lambda}}$. Так как она удовлетворяет уравнению $h_{\bar{\lambda}}-$ $e^{\bar{c}-c} \mu_{1} \overline{h_{\lambda}}=0$ и оператор $1-e^{\bar{c}-c} \mu_{1} Q \Pi$ в $L^{p}(\mathbb{C})$ обратим, то $h_{\bar{\lambda}}=0$ в смысле распределений и, значит, $h$ постоянна по лемме Вейля и теореме Лиувилля.

Так как $h\left(\lambda_{0}\right)=0$, то $h \equiv 0$ и, значит, $\phi_{1} \equiv \phi_{2}$. Лемма доказана.

СлеДСтвиЕ 4.2. Пусть функиии $\mu$ и а удовлетворяют условиям лемм 4.1 u 4.2 и пусть $\phi(\cdot, z)$ - непрерьвное в $\widehat{\mathbb{C}}$ решение уравнения (8) такое, что $\phi_{\bar{\lambda}} \in L^{p}(\mathbb{C}) u \phi(0, z)=z$. Тогда $\phi: \widehat{\mathbb{C}}_{\lambda} \times \mathbb{C}_{z} \rightarrow \mathbb{C}_{w},(\lambda, z) \mapsto w=\phi(\lambda, z)$, ecmъ почти голоморфное движение плоскости $\mathbb{C}_{z}$ над сферой $\left(\widehat{\mathbb{C}}_{\lambda}, 0\right)$, заполняющее все $\widehat{\mathbb{C}} \times \mathbb{C}$, точнее:

- графики всех функиий $\phi(\cdot, z)$ являются J-голоморфными относительно согласованной почти комплексной структуры $J$ с определяющей формой $d w-$ $\mu d \bar{w}-a d \bar{\lambda}$ 
- эти грабики при различных z попарно не пересекаются $u \bigcup_{z}\{(\lambda, w): w=$ $\phi(\lambda, z)\}=\widehat{\mathbb{C}} \times \mathbb{C}$.

ДоказатЕЛЬСтво. Первое утверждение вытекает из определения $J$-голоморфных графиков, второе - из леммы 4.2. Остается последнее. Пусть $\left(\lambda_{0}, w_{0}\right) \in$ $\widehat{\mathbb{C}} \times \mathbb{C}$ и $\psi$ - непрерывное в $\widehat{\mathbb{C}}$ решение уравнения $(8)$ такое, что $\phi_{\bar{\lambda}} \in L^{p}(\mathbb{C})$ и $\psi\left(\lambda_{0}\right)=w_{0}$ (существует по лемме 4.1). Полагая $z_{0}=\psi\left(\lambda_{0}\right)$, по лемме 4.2 получаем, что $\psi=\phi\left(\cdot, z_{0}\right)$, т. е. $w_{0}=\phi\left(\lambda_{0}, z_{0}\right)$. Следствие доказано.

Последнее утверждение о заполнении $\widehat{\mathbb{C}} \times \mathbb{C}$ очень важное (оно означает, что $(\widehat{\mathbb{C}} \times \mathbb{C}, J)$ тривиализуется $J$-голоморфным движением $\phi)$. Условие Липшица на функции $\mu$ и $а$ при этом существенно ослабить нельзя.

ПримеР 4.1. Графики функций $\phi(\cdot, z)$, равных соответственно $z /\left(1+|\lambda|^{2}|z|\right)$ при $|\lambda|<1$ и $z /(1+|z|)$ при $|\lambda| \geqslant 1$, являются $J$-голоморфными относительно согласованной почти комплексной структуры $J$ в $\mathbb{C}^{2}$ с определяющей $(1,0)$-формой $\alpha$, равной $d w+w|w| \lambda d \bar{\lambda}$ при $|\lambda|<1$ и $d w$ при $|\lambda| \geqslant 1$. Они определяют $J$-голоморфное движение над $(\widehat{\mathbb{C}}, 0)$ всей плоскости $\mathbb{C}$, однако пространство этого движения есть множество $\left\{(\lambda, w):|\lambda|<1,|w||\lambda|^{2}<1\right\} \cup((\widehat{\mathbb{C}} \backslash \mathbb{D}) \times \mathbb{D})$.

Разрывность $J$ здесь ни при чем; стандартно усредняя $\alpha$ по $\lambda$, получим тот же эффект, только без явных формул. Существенным здесь является квадратичный рост коэффициента $a$ (при $\mu \equiv 0)$, несовместимый с условием Липшица.

Теперь - о решениях уравнения $(8)$ в круге $\mathbb{D}$, что-то в духе принципа максимума. Обозначим $\|\phi\|_{\partial \mathbb{D}}:=\varlimsup_{|\lambda| \rightarrow 1}|\phi(\lambda)|$.

ЛЕмма 4.3. Пусть $\phi$ - равномерно ограниченное решение класса $W^{1, p}(\mathbb{D})$ уравнения (8) в $\mathbb{D}$, где $p>2$ таково, что $\|\Pi\|_{p}\|\mu\|_{\infty}=: \theta<1$. Предположим, что $a(\lambda, 0) \equiv 0$ и а удовлетворяет условию Липшица по ш с константой, не зависящей от $\lambda \in \mathbb{D}$. Тогда существует константа $M$, зависящая только

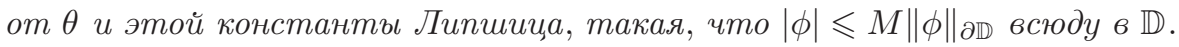

ДокАЗАТЕльСтво. Положим $\nu=\mu(\cdot, \phi) \overline{\phi_{\lambda}} / \phi_{\lambda}$, где $\phi_{\lambda} \neq 0$, и $\nu=0$, где $\phi_{\lambda}=0$, а также $C=a(\cdot, \phi) / \phi$ там, где $\phi \neq 0$, и $C=0$, где $\phi=0$. Тогда $|C| \leqslant\|a(\cdot, w) / w\|_{\infty}$, в частности, $C \in L^{p}(\mathbb{D})$, и $\phi_{\bar{\lambda}}-\nu \phi_{\lambda}=C \phi$. Доопределим $C$ нулем вне $\mathbb{D}$ и положим $c=T(1-\nu \Pi)^{-1} C$. Тогда $c_{\bar{\lambda}}-\nu c_{\lambda}=C$ в $\mathbb{D}$ и $|c| \leqslant M_{1}\|C\|_{\infty}$ с константой $M_{1}$, зависящей только от $\theta$. Так как функции $c, \phi$ ограничены и принадлежат $W^{1, p}(\mathbb{D})$, то функция $\Phi:=e^{-c} \phi$ принадлежит $W^{1, p}(\mathbb{D})$ и удовлетворяет в $\mathbb{D}$ уравнению Бельтрами $\Phi_{\bar{\lambda}}=\nu \Phi_{\lambda}$.

Пусть $g$ есть квазиконформный гомеоморфизм круга $\mathbb{D}$, удовлетворяющий этому же уравнению, $g_{\bar{\lambda}}=\nu g_{\lambda}$. Тогда функция $h=\Phi \circ g^{-1}$ голоморфна в $\mathbb{D}$ и $\|h\|_{\infty} \leqslant\|\Phi\|_{\partial \mathbb{D}}$ по принципу максимума. Следовательно, $\|\Phi\|_{\infty} \leqslant\|\Phi\|_{\partial \mathbb{D}}$, и значит, $\|\phi\|_{\infty} \leqslant e^{2\|c\|_{\infty}}\|\phi\|_{\partial \mathbb{D}}$. Лемма доказана.

СлеДСтвиЕ 4.3. Пусть $\phi_{1}, \phi_{2}$ - решения уравнения (8) такие, как в лемме 4.3. Предположим дополнительно, что н тоже удовлетворяет условию Липшица по w с константой, не зависящей от $\lambda \in \mathbb{D}$. Тогда $\left|\phi_{1}-\phi_{2}\right| \leqslant$ $M_{1}\left\|\phi_{1}-\phi_{2}\right\|_{\partial \mathbb{D}}$ с константой $M_{1}$, зависящей только от $\theta$, констант Липшии,а для $\mu, a u\left\|\left(\phi_{1}\right)_{\bar{\lambda}}\right\|_{L^{p}(\mathbb{D})}$. 
Доказательство. Положим $f=\phi_{2}-\phi_{1}$. Тогда $f_{\bar{\lambda}}-\nu f_{\lambda}=C f$, где $\nu$ равно $\mu\left(\cdot, \phi_{1}\right) \overline{f_{\lambda}} / f_{\lambda}$ при $f_{\lambda} \neq 0$ и 0 при $f_{\lambda}=0$, а $C f$ равно $\left(\mu\left(\cdot, \phi_{2}\right)-\mu\left(\cdot, \phi_{1}\right)\right) \overline{\phi_{1 \lambda}}+$ $a\left(\cdot, \phi_{2}\right)-a\left(\cdot, \phi_{1}\right)$ при $f \neq 0$ и 0 при $f=0$. Тогда $C \in L^{p}(\mathbb{D})$ и остальное - как выше.

Далее нам понадобится следующее локальное свойство.

Лемма 4.4. Пусть $J$ - согласованная почти комплексная структура в $\mathbb{C}^{2}$, удовлетворяющая условиям лемм 4.1 и 4.2 u $S_{j}: w=\phi_{j}(\lambda), j=1,2,-$ различнъе $J$-голоморфнъе графики над областъю $U \subset \mathbb{C}_{\lambda}$. Тогда множество $S_{1} \cap S_{2}$ дискретное и в каждой точке этого множества индекс пересечения поверхностей $S_{1}, S_{2}$ есть иелое положительное число.

(Поверхности $S_{j}$ естественно ориентированы так, что проекция в $U$ coхраняет ориентацию.)

ДоказАтельство. Можно считать, что $U$ ограничена и $\phi_{j} \in W^{1, p}(U)$ с некоторым $p>2$, как выше. Положим $f=\phi_{1}-\phi_{2}$; тогда, как в лемме 4.2 , $f_{\bar{\lambda}}-\mu_{1} \overline{f_{\lambda}}=C f$ с функцией $C \in L^{p}(U)$. Доопределим ее нулем вне $U$ и найдем, как в лемме 4.2 , функцию $c \in W^{1, p}(\mathbb{C})$ такую, что $c_{\bar{\lambda}}-\mu_{2} \overline{c_{\lambda}}=C$. Тогда функция $h=f e^{-c}$ удовлетворяет в $U$ уравнению $h_{\bar{\lambda}}-e^{\bar{c}-c} \mu_{1} \overline{h_{\lambda}}=0$. Положим $\nu=e^{\bar{c}-c} \mu_{1} \overline{h_{\lambda}} / h_{\lambda}$ там, где $h_{\lambda} \neq 0$, и $\nu=0$, где $h_{\lambda}=0$. Тогда $h_{\bar{\lambda}}=\nu h_{\lambda}$ и потому $h$ представляется в виде $H \circ g$, где $H$ - голоморфная функция и $g-$ квазиконформный гомеоморфизм $U$ с коэффициентом Бельтрами $\nu$ (см. [60; теорема 3.30]).

Поэтому нули $h$ в $U$ изолированы, и если $\lambda_{0}-$ единственный нуль $h$ в круге $V:\left|\lambda-\lambda_{0}\right| \leqslant r$, то по теореме Руше приращение аргумента $\Delta_{\partial V} \arg h$ равно $2 \pi k$, где $k \in \mathbb{N}$ - порядок нуля голоморфной функции $H$ в точке $g\left(\lambda_{0}\right)$. А это и есть индекс пересечения $S_{1}$ и $S_{2}$ в точке $\left(\lambda_{0}, \phi_{1}\left(\lambda_{0}\right)\right)$. Лемма доказана.

Наконец, нам понадобится следующее граничное свойство гладкости.

Лемма 4.5. Пусть $\mathbb{D}_{+}=\mathbb{D} \cap\{\operatorname{Im} \lambda>0\}$ и $\phi$ - решение в классе $W^{1, p}\left(\mathbb{D}_{+}\right)$ уравнения (8), в котором $\mu, a \in L^{\infty}\left(\mathbb{D}_{+} \times \mathbb{C}\right)$, причем $\|\mu\|_{\infty}<1 u\|\Pi\|_{p}\|\mu\|_{\infty}<1$. Предположим, что ф ограничено в $\mathbb{D}_{+}$и все его предельные значения на интервале $(-1,1)$ вещественные. Тогда для всякого $r<1$ функиия ф удовлетворяет в $r \mathbb{D}_{+}$условию Гёльдера с показателем $1-2 / p$ и константой, зависящей только от $r, p$, нормы а в $L^{\infty}\left(\mathbb{D}_{+} \times \mathbb{C}\right) u\|\phi\|_{\infty}$.

ДоказАтельство. Положим $f=\phi$ в $\mathbb{D}_{+}$и $f(\lambda)=\overline{\phi(\bar{\lambda})}$ в $\mathbb{D}_{-}:=\mathbb{D} \cap\{\operatorname{Im} \lambda<$ $0\}$. Тогда $f_{\bar{\lambda}}=\overline{\left(\phi_{\bar{\lambda}}\right)(\bar{\lambda})}$ и $f_{\lambda}=\overline{\left(\phi_{\lambda}\right)(\bar{\lambda})}$ в $\mathbb{D}_{-}$. Таким образом, $f$ удовлетворяет

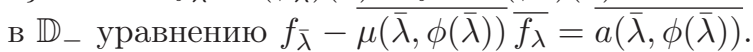

Пусть $g$ - гладкая финитная в $\mathbb{D}$ функция и $D$ - какая-нибудь первая частная производная. Тогда

$$
\int f D g d S=-\int g D f d S+\lim _{\varepsilon \rightarrow 0} \int_{-1}^{1}((g f)(\xi+i \varepsilon)-(g f)(\xi-i \varepsilon)) d \xi=-\int g D f d S,
$$

так как $f(\xi+i \varepsilon)-f(\xi-i \varepsilon)=2 i \operatorname{Im} \phi(\xi+i \varepsilon) \rightarrow 0$ при $\varepsilon \rightarrow 0$. Таким образом, первые частные производные $f$ в смысле распределений в $\mathbb{D}$ принадлежат $L^{p}(\mathbb{D})$, т. е. $f$ принадлежит $W^{1, p}(\mathbb{D})$ и удовлетворяет в $\mathbb{D}$ уравнению $f_{\bar{\lambda}}-\nu \overline{f_{\lambda}}=b$ с равномерно ограниченными $\nu, b$ и с $\|\nu\|_{\infty}=\|\mu\|_{\infty}$. 
Тогда $f(\lambda)=h(\lambda)-\frac{1}{\pi} \int \frac{f_{\bar{\zeta}} d S}{\zeta-\lambda}$, где $h-$ голоморфная в $\mathbb{D}$ функция. Так как $f_{\bar{\lambda}} \in L^{p}(\mathbb{D})$, то плоский интеграл Коши $f-h$ удовлетворяет условию Гёльдера с показателем $1-2 / p$ и константой, зависящей только от $\left\|f_{\bar{\lambda}}\right\|_{p}$ (см. [60; теорема 1.19]).

Пусть $\chi$ - стандартная гладкая финитная функция в $\mathbb{D}$, равная 1 в $r \mathbb{D}$. Доопределяя $f \chi$ равной нулю вне $\mathbb{D}$, мы получаем, что $(f \chi)_{\bar{\lambda}}-\nu \overline{(f \chi)_{\lambda}}=$ $b+f \chi_{\bar{\lambda}}-\nu \overline{f \chi_{\lambda}}=: C$, где $\|C\|_{p}$ оценивается сверху константой, зависящей только от $r$ и норм $b, f$ в $L^{\infty}(\mathbb{D})$. Так как $(f \chi)_{\bar{\lambda}}=(1-\nu Q \Pi)^{-1} C$, то $\left\|(f \chi)_{\bar{\lambda}}\right\|_{p}$ ограничена такой же константой. Остается заметить, что $f \chi=T(f \chi)_{\bar{\lambda}}$, где $T-$ плоский интеграл Коши; так как $(f \chi)_{\bar{\lambda}} \in L^{p}(\mathbb{C})$, то $f \chi$ удовлетворяет условию Гёльдера с показателем $1-2 / p$ и с константой, допускающей указанную оценку. Лемма доказана.

СлеДСтвиЕ 4.4. Пусть $\phi \in W^{1, p}(\mathbb{D})$ - решение уравнения Бельтрами (8)

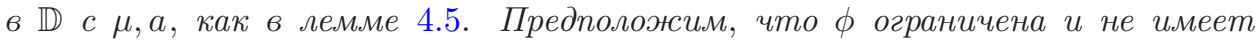
нулей в $\mathbb{D} u|\phi(\lambda)| \rightarrow 1$ при $|\lambda| \rightarrow 1$. Тогда ф удовлетворяет условию Гёльдера с показателем $1-2 / p$ и константой, зависящей только от $p$ и норм $\mu, a$ в $L^{\infty}(\mathbb{D} \times \mathbb{C})$.

ДокАЗАТЕЛЬство. Повторяем доказательство леммы 4.5 с отражением относительно единичной окружности вместо $\mathbb{R}$. В результате функция $f$, продолжающая $\phi$ по симметрии, принадлежит $W^{1, p}(\mathbb{C})$ (возле $\partial \mathbb{D}$ - как в лемме 4.5 ) и удовлетворяет уравнению $f_{\bar{\lambda}}-\nu \overline{f_{\lambda}}=b$ типа (8) уже во всей плоскости, причем функция $\left(1+|\lambda|^{2}\right) b$ равномерно ограничена (при дифференцировании функции $1 / \overline{\phi(1 / \bar{\lambda})}$ появляются множители $1 / \lambda^{2}$ и $1 / \bar{\lambda}^{2}$; подробности см. ниже в доказательстве леммы 4.6).

Остальное следует из леммы 4.5 .

4.2. Краевая задача Римана-Гильберта. Решаемая в этом пункте задача заключается в следующем:

( ) Пусть $T \subset \partial \mathbb{D} \times \mathbb{C}$ - гладкий 2-мерный тор с регулярной проекцией на $\partial \mathbb{D}$ такой, что $T_{\lambda}=\{w:(\lambda, w) \in T\}$ для каждого $\lambda \in \partial \mathbb{D}$ есть гладкая замкнутая жорданова кривая, отделяющая 0 от $\infty$, и пусть $J$ - гладкая согласованная почти комплексная структура в $\mathbb{D} \times \mathbb{C}$. Для данного значения $c \in T_{1}$ доказать существование и описать свойства $J$-голоморфного диска $w=\phi(\lambda)$ такого, что $\phi$ непрерывна и не имеет нулей в $\overline{\mathbb{D}}, \phi(\lambda) \in T_{\lambda}$ для всех $\lambda \in \partial \mathbb{D}$ и $\phi(1)=c$.

(Более общая постановка заключается в том, что $\phi$ имеет нули в заданных точках, но ее решение является легким следствием решения поставленной задачи, см. ниже.)

Если $\alpha=d w-\mu d \bar{w}-a d \bar{\lambda}-$ определяющая форма структуры $J$, то решение задачи $(\star)$ эквивалентно решению уравнения (8)

$$
\phi_{\bar{\lambda}}-\mu(\cdot, \phi) \overline{\phi_{\lambda}}=a(\cdot, \phi)
$$

в классе $C(\overline{\mathbb{D}}) \cap W^{1, p}(\mathbb{D})$ с указанными граничными свойствами.

Что касается гладкости $J$ и $T$, то это требование можно существенно ослабить, не ухудшая результатов. Для приложений нам достаточно гладкости 
и разрешимости в классе $C^{\infty}$, но приводимые ниже доказательства используют лишь гладкость $C^{2}$.

Для обычной комплексной структуры в $\mathbb{C}^{2}$ эта задача решена (см. [24], [54]) при помощи теоремы о неявной функции в банаховых пространствах. Таким же образом можно решать и общую задачу (*), но поставленная задача без дополнительных условий разрешима не для любой (даже интегрируемой $\mathbb{R}$-аналитической) структуры $J$.

Пример 4.2. Почти комплексная структура $J$ в $\mathbb{C}^{2}$ с определяющей формой $d w+w^{2} d \bar{\lambda}-$ интегрируемая и $\mathbb{R}$-аналитическая (нестандартная комплексная структура в $\left.\mathbb{C}^{2}\right)$; см. примеры 1.1-1.3. В качестве $T$ возьмем тор $\partial \mathbb{D} \times\{|w|=2\}$. Предположим, что существует $J$-голоморфный график $w=\phi(\lambda)$ с границей на $T$ и непрерывной в $\overline{\mathbb{D}}$ функцией $\phi$ без нулей. Тогда $\phi_{\bar{\lambda}}=-\phi^{2}$, т. е. $1 / \phi=\bar{\lambda}-h$, где $h$ - непрерывная в $\overline{\mathbb{D}}$ функция, голоморфная в $\mathbb{D}$. Но тогда $|\bar{\lambda}-h|=1 / 2$ на $\partial \mathbb{D}$, что приводит к противоречию, например,

$$
2 \pi=\left|\int_{\partial \mathbb{D}} \bar{\lambda} d \lambda\right|=\left|\int_{\partial \mathbb{D}}(\bar{\lambda}-h) d \lambda\right| \leqslant \pi .
$$

Задача $(\star)$ легко сводится к случаю, когда $T=(\partial \mathbb{D})^{2}-$ стандартный тор (и в этом - преимущество постановки задачи для общих структур $J$ ). Для этого надо просто сделать послойный диффеоморфизм $\overline{\mathbb{D}} \times \mathbb{C}$, переводящий $T$ в $(\partial \mathbb{D})^{2}$ и тождественный в окрестности $\overline{\mathbb{D}} \times 0$ и $\overline{\mathbb{D}} \times \infty$. Структура $J$ в измененных координатах имеет определяющую форму с теми же свойствами гладкости, финитности и т. п., что и исходные $\mu, a$. Поэтому далее мы ограничимся случаем $T=(\partial \mathbb{D})^{2}$.

Лемма 4.6. Пусть $J$ - согласованная почти комплексная структура $в$ $\mathbb{D} \times \mathbb{C}$ с определяющей формой $d w-\mu d \bar{w}-a d \bar{\lambda}$. Предположим, что $\mu \in C^{2}$ в окрестности $0 \times \mathbb{C}, \mu$, а равны нулю при $|w|>R>1$, удовлетворяют условию Липшища по ш с константой, не зависящей от $\lambda \in \mathbb{D}$, причем $\|\mu\|_{\infty}<1$ и $a(\lambda, 0) \equiv 0$. Тогда для любой $c \in \partial \mathbb{D}$ задача $(\star) c T=(\partial \mathbb{D})^{2}$ однозначно разрешима, графики решений $\phi(\lambda, c),|c|=1$, попарно не пересекаются и образуют $J$-голоморфное движение $\partial \mathbb{D}$ над $\mathbb{D}$.

ДокАЗАТЕЛЬСтво. По условию, $\mu \in C^{2}$ в некотором бикруге $|\lambda|<r$, $|w|<R$. Пусть $\chi(\lambda)$ - гладкая функция в $\mathbb{D}$, равная 1 при $|\lambda|<r / 2$ и 0 при $|\lambda|>2 r / 3$, и пусть $\eta=\eta(\lambda, w)$ есть гомеоморфное в $\mathbb{C}_{w}$ решение уравнения Бельтрами $\eta_{\bar{w}}=-\chi \mu \eta_{w}$ такое, что $\eta_{\bar{w}} \in L^{p}(\mathbb{C})$ и $\eta=w+o(1)$ в окрестности $w=\infty$. Тогда $\eta=w$ при $|\lambda|>2 / 3$. Так как $\chi \mu$ имеет компактный носитель, то отображение $\mathbb{D} \ni \lambda \mapsto \chi(\lambda) \mu(\lambda, \cdot) \in L^{\infty}(\mathbb{C})$ дважды гладкое и потому $\eta \in C^{2}(r \mathbb{D} \times \mathbb{C})$ по теореме Альфорса-Берса [1], [2]. Так как $d \eta=\eta_{w}(d w-\mu d w)$ при каждом фиксированном $\lambda \in \frac{r}{2} \mathbb{D}$, то в координатах $\lambda, \eta$ структура $J$ над $\frac{r}{2} \mathbb{D}$ имеет определяющую форму $d \eta-\left(\eta_{\bar{\lambda}}+\eta_{w} a\right) d \bar{\lambda}$, в которой коэффициент при $d \bar{\eta}$ равен нулю. Так как функция $\eta$ голоморфна по $w$ при $|w|>R$, то, по теореме Кёбе, образ круга $|w|<R$ при отображении $w \mapsto \eta$ содержится в круге $|\eta|<4 R$. Чтобы не менять обозначений, мы просто предположим далее, что в исходной определяющей форме $d w-\mu d \bar{w}-a d \lambda$ коэффициент $\mu$ равен нулю при $|\lambda|<r / 2$ и функции $\mu, a$ равны нулю при $|w|>R$. Гладкость $\mu \in C^{2}$ нам далее не понадобится. 
Продолжим $J_{1}$ на все $\mathbb{C}^{2}$ до структуры $J^{\prime}$, исходя из условия, что если $w=f(\lambda)$ есть $J^{\prime}$-голоморфный график над $\mathbb{D}$, то $w=1 / \overline{f(1 / \bar{\lambda})}$ должен быть таковым над $\mathbb{C} \backslash \overline{\mathbb{D}}$. Так как

$$
\left(\frac{1}{\overline{f(1 / \bar{\lambda})}}\right)_{\bar{\lambda}}=\frac{\overline{f_{\bar{\lambda}}(1 / \bar{\lambda})}}{(\overline{\lambda f(1 / \bar{\lambda})})^{2}}, \quad\left(\frac{1}{\overline{f(1 / \bar{\lambda})}}\right)_{\lambda}=\frac{\overline{f_{\lambda}(1 / \bar{\lambda})}}{(\overline{\lambda f(1 / \bar{\lambda})})^{2}}
$$

при $|\lambda|>1$, то это условие выполняется, если в качестве определяющей формы над $\mathbb{C} \backslash \overline{\mathbb{D}}$ взять

$$
d w-\overline{\left(\frac{1}{\bar{\lambda}}, \frac{1}{\bar{w}}\right)}\left(\frac{w}{\bar{w}}\right)^{2} d \bar{w}-\overline{a\left(\frac{1}{\bar{\lambda}}, \frac{1}{\bar{w}}\right)}\left(\frac{w}{\bar{\lambda}}\right)^{2} d \bar{\lambda} .
$$

Обозначим через $a^{\prime}(\lambda, w)$ коэффициент этой формы при $\bar{\lambda}$. Тогда $a^{\prime}(\lambda, 0) \equiv 0$. Так как функция $a(1 / \lambda, \eta)$ удовлетворяет условию Липшица по $\eta$, то она дифференцируема почти всюду и ее первые частные производные по $\eta, \bar{\eta}$ не превосходят константы Липшица. Так как

$$
\left|a^{\prime}(\lambda, w)_{\bar{w}}\right|=\left|\frac{a_{\bar{w}}(1 / \bar{\lambda}, 1 / \bar{w})}{\lambda^{2}}\right|, \quad a^{\prime}(\lambda, w)_{w}=\overline{a_{\bar{w}}\left(\frac{1}{\bar{\lambda}}, \frac{1}{\bar{w}}\right)} \frac{w^{2}}{\bar{\lambda}^{2}}+\overline{a\left(\frac{1}{\bar{\lambda}}, \frac{1}{\bar{w}}\right)} \frac{2 w}{\bar{\lambda}^{2}}
$$

и $|a(1 / \bar{\lambda}, 1 / \bar{w})| \leqslant C /|w|$, то $a^{\prime}$ удовлетворяет условию Липшица по $w$ с константой, не зависящей от $\lambda \in \mathbb{C} \backslash \overline{\mathbb{D}}$.

Таким образом, коэффициенты определяющей формы структуры $J^{\prime}$ в $\mathbb{C}^{2}$ удовлетворяют условиям следствия 4.2 , поэтому решения $f(\cdot, z)$ уравнения $(8)$ с так продолженными $\mu, a$ и с $f(1, z)=z$ определяют $J^{\prime}$-голоморфное движение, заполняющее $\mathbb{C}^{2}, f(\lambda, 0) \equiv 0$. Если $f$ - одно из этих решений, то на графике $w=1 / \overline{f(1 / \bar{\lambda})}$ имеет место равенство

$$
\left(\frac{1}{\overline{f(1 / \bar{\lambda})}}\right)_{\bar{\lambda}}+\overline{\mu\left(\frac{1}{\bar{\lambda}}, f\left(\frac{1}{\bar{\lambda}}\right)\right)}\left(\frac{f(1 / \bar{\lambda})}{\overline{f(1 / \bar{\lambda})}}\right) \cdot \overline{\left(\frac{1}{f(1 / \bar{\lambda})}\right)_{\lambda}}=\frac{\overline{a(1 / \bar{\lambda}, f(1 / \bar{\lambda}))}}{\overline{(\lambda f(1 / \bar{\lambda}))^{2}}},
$$

т. е. $1 / \overline{f(1 / \bar{\lambda})}$ - тоже одна из построенных функций, а именно $f(\lambda, 1 / \overline{f(1)})$ по теореме единственности. Таким образом, при $|z|=1$ имеет место равенство $f(\lambda, z)=1 / \overline{f(1 / \bar{\lambda}, z)}$, в частности, $|f(\lambda, z)| \equiv 1$ при $|\lambda|=1$, т. е. $f(\cdot, z)$ - решение задачи $(\star)$ для структуры $J^{\prime}$ при $|z|=1$. Графики этих функций над $\widehat{\mathbb{C}}$ по лемме 4.2 попарно не пересекаются и образуют $J^{\prime}$-голоморфное движение $\mathbb{C}_{z}$ над $\left(\mathbb{C}_{\lambda}, 1\right)$. Так как $f(\lambda, \cdot) \equiv 0$, то функции $f(\lambda, z)$ при $z \neq 0$ не имеют нулей. Таким образом, $\phi(\cdot, c):=f(\cdot, c),|c|=1,-$ искомые решения. Лемма доказана.

СЛЕДСТВИЕ 4.5. При условиях леммы 4.6, задача (*) с T $=\partial \mathbb{D} \times|c| \partial \mathbb{D}$ однозначно разрешима для каждого $c \in \mathbb{C}$. Решения непрерывно зависят от $c$ относительно метрики в $C(\overline{\mathbb{D}})$. Графики этих решений над $\overline{\mathbb{D}}$ при различньх с попарно не пересекаются и заполняют все $\overline{\mathbb{D}} \times \mathbb{C}$.

ДокАзАтельство. При $t>0$ функции $\mu_{t}=\mu(\cdot, t w)$ и $a(\cdot, t w) / t$ удовлетворяют условиям леммы 4.6 и поэтому для всякого $b \in \partial \mathbb{D}$ существует единственное решение $\psi$ уравнения $\psi_{\bar{\lambda}}-\mu_{t} \overline{\psi_{\lambda}}=a_{t}$, непрерывное в $\overline{\mathbb{D}}$ и класса $W^{1, p}(\mathbb{D})$ такое, что $|\psi|=1$ на $\partial \mathbb{D}$ и $\psi(1)=b$. А тогда функция $\phi_{c}:=t \psi, c=t b$, является решением уравнения (8) с граничными данными $\left|\phi_{c}\right|=|c|, \phi_{c}(1)=c$, причем графики этих решений при фиксированном $t=|c|$ попарно не пересекаются. 
По следствию 4.3, семейство решений уравнения (8) равностепенно непрерывно. Из этого и теоремы единственности (лемма 4.2) очевидно вытекает непрерывность отображения $c \longmapsto \phi_{c} \in C(\overline{\mathbb{D}})$.

По теореме единственности, $\phi_{c} \equiv c$ при $|c|>R$. Графики функций $\phi_{c^{\prime}}$ и $\phi_{c}$ при $\left|c^{\prime}\right|<R$ и больших $|c|$ не пересекаются. Так как границы графиков при различных $c$ тоже не пересекаются, то из этого следует, что индексы пересечения таких графиков равны нулю. Так как индекс пересечения в каждой возможной общей точке различных графиков положителен по лемме 4.4, то графики различных $\phi_{c}$ вообще не пересекаются.

Наконец, отображение $c \mapsto \phi_{c}(\lambda)$ при каждом фиксированном $\lambda$ непрерывно и взаимно однозначно, образ содержит окрестность бесконечности и потому совпадает с $\mathbb{C}$. Следствие доказано.

СлЕДСтвиЕ 4.6. Если $Ј$ удовлетворяет условиям леммы и $\lambda_{1}, \ldots, \lambda_{n}-$ заданные точки в $\mathbb{D}$, выписанные с учетом кратностей, то для любого $c \in \partial \mathbb{D}$ существует единственная непрерьвная в $\overline{\mathbb{D}}$ функиия $f$ с J-голоморфным графиком над $\mathbb{D}$ такая, что $|f|=1$ на $\partial \mathbb{D}, f(1)=$ с $и f\left(\lambda_{\nu}\right)=0, \nu=1, \ldots, n$ (с нулями соответствующих кратностей), причем $f(\lambda) \neq 0$, если $\lambda \neq \lambda_{\nu}$, $\nu=1, \ldots, n$.

ДокАзАтельство. Условия не меняются при дробно-линейном автоморфизме $\mathbb{D}$, близком к тождественному, поэтому можно считать, что все $\lambda_{\nu}$ отличны от нуля. Обозначим через $b$ произведение Бляшке $\prod_{1}^{n} \frac{\lambda-\lambda_{\nu}}{1-\overline{\lambda_{\nu}} \lambda}$ и рассмотрим в $\mathbb{D} \times \mathbb{C}$ почти комплексную структуру $J_{1}$ с определяющей формой $d w-\mu_{1} d \bar{w}-(a(\cdot, w b) / b) d \bar{\lambda}$, где $\mu_{1}(\cdot, w):=\mu(\cdot, w b) \overline{b^{\prime}} / b^{\prime}$. Структура $J_{1}$ удовлетворяет всем условиям леммы 4.6, и потому существует единственная непрерывная в $\overline{\mathbb{D}}$ функция $g$ без нулей, с $J_{1}$-голоморфным графиком над $\mathbb{D}$ такая, что $|g|=1$ на $\partial \mathbb{D}$ и $g(1)=c / b(1)$. А тогда $f:=b g-$ искомая функция. Следствие доказано.

Почти комплексную структуру $J$ в $\mathbb{D} \times \mathbb{C}$ назовем диск-выпуклой над $\partial \mathbb{D}$, если выполняется следующее условие: существует $r_{0}>0$ такое, что для всякого $r>r_{0}$ найдется $R>0$ такое, что если функция $f$ непрерывна в $\overline{\mathbb{D}}$, ее график $J$-голоморфен над $\mathbb{D}$ и $r_{0}<|f| \leqslant r$ на $\partial \mathbb{D}$, то $|f|<R$ всюду в $\mathbb{D}$.

Теорема 4.1. Пусть $J$-диск-выпуклая над плексная структура в $\mathbb{D} \times \mathbb{C}$ с определяющей формой $d w-\mu d \bar{w}-a d \bar{\lambda}$. Предположим, что $\mu \in C^{2}$ в окрестности $0 \times \mathbb{C}, a \in L_{\mathrm{loc}}^{\infty} u$ для всякого $R>0$ существует $\theta_{R}<1$ такое, что $|\mu|<\theta_{R}$ при $|w|<R$. Предположим также, что $\mu$ и а локально по $w$ удовлетворяют условиям Липиица с константами, не зависящими от $\lambda \in \mathbb{D}, u a(\lambda, 0) \equiv 0$. Тогда для всякого $z \in \mathbb{C} \backslash 0$ существует единственныи $J$-голоморфный над $\mathbb{D}$ график $w=\phi(\lambda, z), \lambda \in \overline{\mathbb{D}}$, с непрерывной

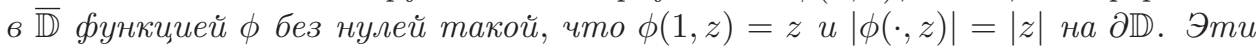
графики попарно не пересекаются и их обгединение равно $(\partial \mathbb{D} \times \mathbb{C}) \cup \mathscr{D}$, где $\mathscr{D}$ - некоторая область в $\mathbb{D} \times \mathbb{C}$ с односвязными слоями $\mathscr{D}_{\lambda}$.

ДокАЗАтЕльство. Фиксируем $r>r_{0}$, и пусть $R$ - соответствующее ему число из определения диск-выпуклости над $\partial \mathbb{D}$. Пусть $\chi(w)$ - гладкая функция, равная 1 при $|w|<2 R$ и 0 при $|w|>3 R$. 
Так как $a(\lambda, 0) \equiv 0$, то диск $\mathbb{D} \times 0$ является $J$-голоморфным. Согласно следствию 4.5, существует единственное решение $\phi_{c}$ уравнения

$$
\phi_{\bar{\lambda}}-\chi(\phi) \mu(\cdot, \phi) \overline{\phi_{\lambda}}=\chi(\phi) a(\cdot, \phi),
$$

непрерывное в $\overline{\mathbb{D}}$ и класса $W^{1, p}(\mathbb{D})$, с граничными данными $\left|\phi_{c}\right| \equiv|c|$ на $\partial \mathbb{D}$ и $\phi_{c}(1)=c$. Если модуль $|c|$ достаточно мал, то $\left|\phi_{c}\right|<R$ по лемме 4.3 и потому $\phi_{c}$ - решение уравнения (8). Обозначим через $R_{0}$ верхнюю грань чисел $t>0$ таких, что $\left|\phi_{c}\right|<2 R$ для всех $c \in t \mathbb{D}$. Если $R_{0}<r$, то на самом деле $\left|\phi_{c}\right| \leqslant R$ при $c \in t \mathbb{D}, t \leqslant R_{0}$, - по определению диск-выпуклости над $\partial \mathbb{D}$. В частности, это верно при $t=R_{0}$. Так как решения уравнения (9) непрерывно зависят от $c$, то существует $\varepsilon>0$ такое, что $\left|\phi_{c}\right|<2 R$ для всех $c$ таких, что $|c|<R_{0}+\varepsilon$. Это противоречие с определением $R_{0}$ показывает, что $R_{0} \geqslant r$, т. е. $\phi_{c}-$ решения уравнения (8) и при $|c|=r$. Таким образом, существование доказано.

Остальное - как в следствии 4.5, в частности, каждое отображение $c \longmapsto$ $\phi_{c}(\lambda), \lambda \in \mathbb{D},-$ непрерывное и однолистное, поэтому его образ $\mathscr{D}_{\lambda}$ односвязный. Теорема доказана.

Структуру $J$ в $\mathbb{D} \times \mathbb{C}$ назовем трансверсально диск-выпуклой, если для всякого $R>0$ найдется $R^{\prime} \geqslant R$ такое, что если $w=f(\lambda)$ - произвольный график, $J$-голоморфный над некоторым кругом $U \subset \mathbb{D}$, функция $f$ непрерывна в $\bar{U}$ и $|f| \leqslant R$ на $\partial U$, то $|f| \leqslant R^{\prime}$ в $U$. (Если $J$ - комплексная структура, то это есть условие трансверсальной диск-выпуклости голоморфного семейства $(\mathbb{D} \times \mathbb{C}, J) \rightarrow \mathbb{D}$.)

ДоПолнение 4.1. Предположим, что структура J, дополнителъно к условиям теоремы, гладкая и трансверсально диск-выпуклая. Тогда область Д из теоремь универсальная: если функиия $f \in C(\overline{\mathbb{D}}) \cap C^{2}(\mathbb{D})$ не имеет нулей и ее график над $\mathbb{D}$ является $J$-голоморфным, то этот график принадлежит $\mathscr{D}$; более того, если $R=\max _{\partial \mathbb{D}}|f|, \operatorname{mo}|f(\lambda)| \leqslant \max \{|\phi(\lambda, z)|:|z| \leqslant R\}$ для всех

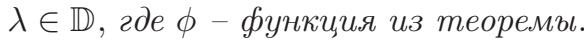

ДоказАтельство. Диффеоморфизм $(\lambda, w) \mapsto\left(\lambda, w^{\prime}:=w / f(\lambda)\right)$ класса $C^{2}$ переводит график $w=f(\lambda)$ в график $w^{\prime}=1$. Образ $J^{\prime}$ структуры $J$ относительно этого преобразования удовлетворяет всем условиям теоремы над любым кругом $r \mathbb{D}, r \in(0,1)$. Фиксируем такое $r$. По теореме, существует $J^{\prime}$-голоморфное движение $\psi^{\prime}: r \mathbb{D} \times \mathbb{C} \rightarrow \mathbb{C}$ над $(r \mathbb{D}, r)$ такое, что $\left|\psi\left(\lambda, z^{\prime}\right)\right| \equiv\left|z^{\prime}\right|$ при $|\lambda|=r$. Прообраз $\psi$ этого движения является $J$-голоморфным движением над $(r \mathbb{D}, r)$, включающим график $f$ над $r \mathbb{D}$. Положим $f_{t}:=\psi(\cdot, t)$; тогда $f_{1}=f, f_{0}=0,\left|f_{t}(\lambda)\right|=t|f(\lambda)|$ при $|\lambda|=r$ и графики всех $f_{t}$ являются $J$-голоморфными над $r \mathbb{D}$.

Обозначим через $E$ множество таких $t \in[0,1]$, что график $f_{t}$ принадлежит $\mathscr{D}$. Так как $f_{t}$ непрерывны на $r \overline{\mathbb{D}}$, то $E$ открыто в $[0,1]$. Обозначая $R_{r}:=\max _{r \partial \mathbb{D}}|f|$, мы получаем, что графики $f_{t}, t \in E$, над $r \partial \mathbb{D}$ не пересекают $w=\phi(\lambda, z)$ с $|z|>R_{r}$. Если $t \in E$, то $f_{t}(r \overline{\mathbb{D}}) \subset \mathscr{D}$ и потому существует $R(t)>0$ такое, что график $f_{t}$ не пересекает графики $w=\phi(\lambda, z)$ с $|z|>R(t)$. Так как индексы пересечения $J$-голоморфных кривых положительны (лемма 4.4) и не меняются при малых деформациях, то $\left|f_{t}(\lambda)\right| \leqslant \max \left\{|\phi(\lambda, z)|:|z| \leqslant R_{r}\right\}$ для всех $\lambda \in \mathbb{D}$. Отсюда очевидно следует, что предельные точки множества $E$ 
тоже принадлежат $E$ и, значит, $E=[0,1]$. В частности, график $f$ принадлежит $\mathscr{D},|f(\lambda)| \leqslant \max \left\{|\phi(\lambda, z)|:|z| \leqslant R_{r}\right\}$ и это верно для всех $r \in(0,1)$, что и доказывает утверждение.

В этом смысле $\mathscr{D}$ является дополнительной диск-выпуклой оболочкой множества $\partial \mathbb{D} \times \mathbb{C}$ в $(\mathbb{D} \times \mathbb{C}, J)$.

Как видно из доказательства, свойство $|\phi(\lambda, z)| \equiv|z|$ движения $\phi$ удобно, но не является существенным, - важна только непрерывность вплоть до границы. Поэтому, если $\psi: \overline{\mathbb{D}} \rightarrow \mathbb{C}$ - другое непрерывное движение, $J$-голоморфное над $\mathbb{D}$, и $\mathscr{D}^{\prime}-$ заполняемая им область над $\mathbb{D}$, то по той же причине все диски $w=\phi(\lambda, z)$ принадлежат $\mathscr{D}^{\prime}$ и, следовательно, $\mathscr{D}^{\prime}=\mathscr{D}$ не зависит от выбора такого движения.

Область $\mathscr{D}$ в теореме совсем не обязана совпадать с $\mathbb{D} \times \mathbb{C}$.

Пример 4.3. Пусть $J$ - согласованная почти комплексная структура в $\mathbb{D} \times \mathbb{C}$ с $(1,0)_{J}$-формами $d \lambda$ и $d\left(w \sqrt{2-\left|\lambda^{2}\right|} / \sqrt{1+|w|^{2}}\right)$. Тогда $(\mathbb{D} \times \mathbb{C}, J)-$ комплексное многообразие, биголоморфно послойно эквивалентное области $\{(\lambda, z)$ : $\left.|\lambda|<1,|\lambda|^{2}+|z|^{2}<2\right\}$ в обычном $\mathbb{C}^{2}$. J-голоморфные графики из теоремы соответствуют при этом плоским графикам $z=c$ над $\mathbb{D}$, заполняющим единичный бикруг. Следовательно, область $\mathscr{D}$ из теоремы в этом примере совпадает с областью $\left\{(\lambda, w):|w|<1 / \sqrt{1-|\lambda|^{2}}\right\}$.

Этот пример показывает, что если $\mathbb{D} \times \mathbb{C}$ в теореме все-таки заполняется $J$-голоморфным движением, то структура $J$ "на бесконечности" не только диск-выпуклая, но в определенном смысле и диск-вогнутая (так сказать, Леви-плоская); подробнее см. п. 5.2.

ЗАмЕчАниЕ 4.1. В приложениях теоремы бывает удобным иметь аналогичное $J$-голоморфное движение не в $\mathbb{D} \times \mathbb{C}$, а в $\mathbb{D} \times \mathbb{D}$ с согласованной почти комплексной структурой $J$. Это очевидно сводится к уже доказанному диффеоморфизмом $(\lambda, w) \mapsto\left(\lambda, w / \sqrt{1-|w|^{2}}\right)$, который является биголоморфным отображением почти комплексного многообразия $\left(\mathbb{D}^{2}, J\right)$ на $\left(\mathbb{D} \times \mathbb{C}, J^{\prime}\right)$ с соответствующей $J^{\prime}$. Структура $J^{\prime}$ удовлетворяет условиям теоремы, если структура $J$ диск-выпуклая над $\partial \mathbb{D}$ (т. е. для всякого $r \in\left(r_{0}, 1\right)$ найдется $R \in(0,1)$ такое, что если $w=f(\lambda)$ - непрерывный в $\overline{\mathbb{D}}$ график, $J$-голоморфный над $\mathbb{D}$, и $r_{0}<|f|<r$ на $\partial \mathbb{D}$, то $|f|<R$ в $\left.\mathbb{D}\right)$ и коэффициенты определяющей формы $d w-\mu d \bar{w}-a d \bar{\lambda}$ типа $(1,0)_{J}$ аналогичные: $a, \mu$ локально по $w$ удовлетворяют условию Липшица с константой, не зависящей от $\lambda, \mu \in C^{2}$ в окрестности $0 \times \mathbb{D}, a \in L_{\mathrm{loc}}^{\infty}$ и для всякого $r \in(0,1)$ существует константа $\theta_{r}<1$ такая, что $|\mu|<\theta_{r}$ при $|w|<r$. Тогда прообраз $\phi$ построенного в теореме движения $\phi^{\prime}$ будет $J$-голоморфным движением, непрерывным над $\overline{\mathbb{D}}$ и заполняющим $(\partial \mathbb{D} \times \mathbb{D}) \cup \mathscr{D}$, где $\mathscr{D}$ - некоторая область в $\mathbb{D}^{2}$ с односвязными слоями $\mathscr{D}_{\lambda}$.

Базовую точку движения $\phi$ тоже бывает удобнее сместить в $\lambda=0$, заменяя $\phi(\lambda, \cdot)$ на $J$-голоморфное движение $\psi(\lambda, z)=\phi\left(\lambda, \phi(0, \cdot)^{-1}(z)\right), z \in \mathscr{D}_{0}$, с теми же $J$-голоморфными графиками.

Пусть $G$ - некоторая группа послойных гомеоморфизмов $\mathbb{D} \times \mathbb{D} \rightarrow \mathbb{D}$ вида $g(\lambda, z)=\left(\lambda, g_{\lambda}(z)\right)$. Движение $\phi: \mathbb{D} \times E \rightarrow \mathbb{D}$ множества $E \subset \mathbb{D}$ над $(\mathbb{D}, 0)$ называется инвариантным относительно $G\left(G\right.$-инвариантным), если $g_{\lambda} \circ \phi(\lambda, \cdot)=$ $\phi(\lambda, \cdot) \circ g_{0}$ для всякого $g \in G$. 
Следующее утверждение о продолжении $J$-голоморфных движений - в духе теоремы Слодковского (см. п. 3.3).

СледСтвиЕ 4.7. Пусть $J$ - почти комплексная структура в $U \times \mathbb{D}, U \supset \overline{\mathbb{D}}$, со всеми свойствами, описанными в замечании 4.1, и $G$ - некоторая группа послойных $J$-голоморфных преобразований $U \times \mathbb{D}$, элементы которой, кроме тождественного, не имеют неподвижных точек. Пусть $\phi^{0}: U \times \mathbb{D} \rightarrow \mathbb{D}$ есть $J$-голоморфное движение над $(\mathbb{D}, 0)$, заполняющее $U \times \mathbb{D}, E-$ подмножество в $\mathbb{D}$ такое, что сужение $\phi^{0} \mid U \times E$ инвариантно относительно $G, u E^{\prime}$ некоторое счетное подмножество в $\mathbb{D}$. Тогда существует $J$-голоморфное $u G$-инвариантное движение $\phi: \overline{\mathbb{D}} \times(E \cup \widetilde{E}) \rightarrow \mathbb{D}$, где $\widetilde{E}:=\bigcup_{g \in G} g_{0}\left(E^{\prime}\right)$, совпадающее с $\phi^{0}$ на $\overline{\mathbb{D}} \times E$.

ДоказАТЕльство. Не меняя $\widetilde{E}$, можно уменьшить $E^{\prime}=\left\{z_{n}\right\}_{1}^{\infty}$ так, что $\left(0 \times E^{\prime}\right) \cap g\left(0 \times E^{\prime}\right)$ пусто для каждого $g \in G \backslash \mathrm{id}$.

Так как $E$ дискретное и графики движения $\phi^{0}$ попарно не пересекаются, то существует послойный диффеоморфизм $h^{0}$ области $U \times D$, переводящий пространство движения $\phi^{0} \mid U \times E$ в $U \times E$ и сохраняющий все свойства $J$ из замечания над любым кругом $R \mathbb{D} \Subset U$. Образ $J$ относительно $h^{0}$ обозначим через $J_{1}$. Пусть $\left\{R_{n}\right\}$ - произвольная убывающая последовательность чисел $R_{n}>1$ такая, что $R_{1} \mathbb{D} \Subset U$ и $\lim R_{n}>1$. Так как движение $\phi^{0}$ заполняет $U \times \mathbb{D}$ по условию, то его образ $\phi^{0} \circ\left(h^{0}\right)^{-1}$ тоже заполняет $U \times \mathbb{D}$.

Пусть $\phi^{1}$ есть $J_{1}$-голоморфное движение над $R_{1} \mathbb{D}$, непрерывное над $R_{1} \overline{\mathbb{D}}$ и состоящее из решений краевой задачи (*) для стандартных торов $R_{1} \partial \mathbb{D} \times r \partial \mathbb{D}$, $r \in(0,1)$. По построению, пространство движения $\phi^{1}$ содержит множество $R_{1} \mathbb{D} \times E$. Согласно дополнению 4.1 к теореме $4.1, \phi^{1}$ тоже заполняет $R_{1} \mathbb{D} \times \mathbb{D}$, и, значит, существует $J_{1}$-голоморфный диск $w^{\prime}=\phi^{1}\left(\lambda, z_{1}^{\prime}\right)$, содержащий точку $\left(0, z_{1}^{\prime}\right)=h^{0}\left(0, z_{1}\right)$.

Положим $\phi\left(\lambda, z_{1}\right):=\phi^{1} \circ\left(h^{0}\right)^{-1}\left(\lambda, z_{1}^{\prime}\right)$. Тогда диск $w=\phi\left(\lambda, z_{1}\right)$ не пересекает диски $w=\phi^{0}(\lambda, z), z \in E$. Пусть $g \in G$ и $z_{1}^{g}:=g_{0}\left(z_{1}\right) \in \widetilde{E}$. Положим $\phi\left(\lambda, z_{1}^{g}\right):=$ $g_{\lambda} \circ \phi\left(\lambda, z_{1}\right)$. Так как движение $\phi^{0} \mid U \times E$ инвариантно относительно $G$, то графики $w=\phi\left(\lambda, z_{1}^{g}\right)$ тоже не пересекают пространство движения $\phi^{0} \mid R_{1} \mathbb{D} \times E$ (поскольку это верно для $g=\mathrm{id}$ по построению).

Если для некоторых $\lambda_{0} \in R_{1} \overline{\mathbb{D}}$ и $g, g^{\prime} \in G$ выполнено равенство $\phi\left(\lambda_{0}, z_{1}^{g}\right)=$ $\phi\left(\lambda_{0}, z^{g_{1}^{\prime}}\right)$, то $\left(\lambda_{0}, \phi\left(\lambda_{0}, z_{1}^{g}\right)\right)$ - неподвижная точка отображения $g^{-1} \circ g^{\prime} \in G$ и, значит, $g=g^{\prime}$ по условию на $G$. Таким образом мы продолжили движение $\phi^{0} \mid R_{1} \mathbb{D} \times E$ до $J$-голоморфного и $G$-инвариантного движения множества $E_{1}$, содержащего $E \cup z_{1}$.

Множество $E_{1}$ такое же, как $E$. По доказанному, построенное движение $E_{1}$ над $\left(R_{1} \mathbb{D}, 0\right)$ продолжается до $J$-голоморфного и $G$-инвариантного движения над $\left(R_{2} \mathbb{D}, 0\right)$ множества $E_{2} \supset E_{1} \cup z_{2}-$ и т. д. по индукции.

Функция на $\overline{\mathbb{D}} \times(E \cup \widetilde{E})$, равная построенной $\phi$ на $R_{n} \mathbb{D} \times E_{n}$, определяет искомое движение $E \cup \widetilde{E}$ над $(\overline{\mathbb{D}}, 0)$. Следствие доказано.

Вопрос о продолжении построенного движения на замыкание множества $E \cup \widetilde{E}$ немного выпадает из дальнейшей тематики, и мы оставляем его открытым. 


\section{5. Униформизация некоторых семейств}

5.1. Компактные поверхности с проколами. Пусть $\pi: M \rightarrow B$ - голоморфное семейство компактных римановых поверхностей рода $\geqslant 2, \lambda_{0} \in B-$ базовая точка, $f_{0}: \mathbb{D} \rightarrow M_{0}$ - универсальное накрытие и $\Gamma$ - соответствующая накрывающая группа. Если $B$ конечномерное и односвязное, то по теореме Гротендика $([32], 1960)$, существуют голоморфные отображения $\kappa: B \rightarrow T(\Gamma)$ и $H: M \rightarrow V(\Gamma)$ такие, что П $H=\kappa \circ \pi$, где $\Pi: V(\Gamma) \rightarrow T(\Gamma)-$ универсальное голоморфное семейство над пространством Тейхмюллера фуксовой группы $\Gamma$ (см. п. 3.1). Примерно в то же время Берс доказал униформизуемость этого универсального семейства [7], [8], определил и описал расслоение $F(\Gamma) \rightarrow T(\Gamma)$. Эти два результата вместе составляют теорему Гротендика-Берса:

Любое голоморфное семейство компактных римановых поверхностей рода $\geqslant 2$ над односвязным комплексным многообразием канонически униформизуеMO.

(Размерность базы $B$ не существенна, классифицирующее отображение $\kappa$ определено и голоморфно и для бесконечномерных многообразий, см. ниже.)

Эрл [19] распространил эту теорему на семейства компактных поверхностей с проколами, голоморфно зависящими от точек базы. Точнее, он доказал следующее утверждение.

Пусть $\hat{\pi}: \widehat{M} \rightarrow B$ - голоморфное семейство компактных римановых поверхностей рода $\geqslant 2$ над односвязным многообразием, $s_{1}, \ldots, s_{n}$ - глобальные голоморфные сечения проекции $\hat{\pi}$ с попарно не пересекающимися образами, $M=\widehat{M} \backslash \bigcup_{j=1}^{n} s_{j}(B)$ u $\pi=\hat{\pi} \mid M$. Тогда семейство $\pi: M \rightarrow B$ канонически униформизуемо.

Доказательства этих утверждений легко следуют из результатов раздела 3 (см. ниже теорему 5.1), согласно которым достаточно доказать локальную тривиализуемость описанных выше семейств голоморфными движениями (теорема 3.1). На этом пути условия в теореме Эрла можно слегка ослабить, а утверждение - усилить, но для этого предварительно надо доказать следующую в общем понятную лемму, для которой, к сожалению, не нашлось подходящей ссылки.

Лемма 5.1. Пусть $E$ - замкнутое подмножество бикруга $\mathbb{D}^{2}$, не пересекающее $\mathbb{D} \times \partial \mathbb{D}$ и такое, что все слои $E_{\lambda}, \lambda \in \mathbb{D}$, вполне разрьвнье. Предположим, что семейство плоских областей $\mathbb{D}^{2} \backslash E \rightarrow \mathbb{D}$ топологически тривиальное и трансверсально диск-выпуклое. Тогда Е является обгединением попарно непересекающихся голоморфных графиков, образующих голоморфное движение $E_{0}$ над $(\mathbb{D}, 0)$.

ДоказАтельство. Для каждого круга $U \Subset \mathbb{D}$ обозначим через $E_{U}$ множество $E \cap(\bar{U} \times \mathbb{D})$. Пусть $\widehat{E}_{U}$ есть множество точек $\left(\lambda^{\prime}, w^{\prime}\right) \in \bar{U} \times \mathbb{D}$ таких, что, для любого многочлена $P(\lambda)$, величина $\left|w^{\prime}-P\left(\lambda^{\prime}\right)\right|$ не превосходит максимума $|w-P(\lambda)|$ на вертикальной границе $b E_{U}:=E \cap(\partial U \times \mathbb{D})$ множества $E_{U}$. Покажем, что $E_{U} \subset \widehat{E}_{U}$. 
Предположим, что множество $E_{U} \backslash \widehat{E}_{U}$ непусто. Тогда найдутся многочлен $P$ и точка $\left(\lambda_{0}, w_{0}\right) \in E_{U} \backslash \widehat{E}_{U}$ такие, что

$$
\left|w_{0}-P\left(\lambda_{0}\right)\right|=\max _{E_{U}}|w-P|>\max _{b E_{U}}|w-P| .
$$

Пусть $\sigma:=\left\{\lambda \in U: \max _{E_{\lambda}}|w-P|=\left|w_{0}-P\left(\lambda_{0}\right)\right|\right\}$. Тогда $\sigma-$ компакт и если $\lambda_{0}$ - центр круга $U$, то существует точка $\lambda_{1} \in \sigma$ такая, что $\left|\lambda_{1}-\lambda_{0}\right| \geqslant\left|\lambda-\lambda_{0}\right|$ для всех $\lambda \in \sigma$. Тогда $\left|\lambda+\lambda_{1}-2 \lambda_{0}\right|<2\left|\lambda-\lambda_{0}\right|$ для всех $\lambda \in \sigma \backslash \lambda_{1}$ и поэтому существует $\alpha \in \mathbb{R}$ такое, что максимум модуля функции $g(\lambda, w):=w-P(\lambda)-$ $e^{i \alpha}\left(\lambda+\lambda_{1}-2 \lambda_{0}\right)$ на $E_{U}$ достигается только на множестве $E_{\lambda_{1}}$, скажем, в точке $\left(\lambda_{1}, w_{1}\right) \in E_{\lambda_{1}}$. В частности, $\left|g\left(\lambda_{1}, w_{1}\right)\right| \geqslant(1+\delta) \max _{b E_{U}}|g|$ с некоторым $\delta>0$. А тогда границы голоморфных графиков $S_{\varepsilon}: g(\lambda, w)=(1+\varepsilon) g\left(\lambda_{1}, w_{1}\right)$ при $0 \leqslant \varepsilon \leqslant \delta / 2$ и достаточно малом $\delta$ образуют компакт в $\mathbb{D}^{2}$, не пересекающий $E$, все $S_{\varepsilon}$ с $\varepsilon>0$ его тоже не пересекают, а предельный график $S_{0}$ содержит точку $\left(\lambda_{1}, w_{1}\right) \in E_{U}$. Это противоречие с условием трансверсальной диск-выпуклости показывает, что на самом деле $E_{U} \subset \widehat{E}_{U}$ для любого круга $U \Subset \mathbb{D}$.

Теперь докажем утверждение в ключевом случае, когда $\# E_{\lambda} \equiv 1$, т. е. $E-$ непрерывный график $w=f(\lambda)$. Тогда, по доказанному, для любого круга $U \Subset \mathbb{D}$ и любого многочлена $P(\lambda)$ справедливо неравенство $|(f-P)(\lambda)| \leqslant$ $\max _{\partial U}|f-P|$ для всех $\lambda \in U$.

Фиксируем произвольный круг $U \Subset \mathbb{D}$. Функции вида $c f-h$, где $c-$ константа и $h$ голоморфна в $U$ и непрерывна в $\bar{U}$, образуют замкнутое подпространство в $\partial U$, содержащее 1 . Линейный функционал $c f-h \mapsto(c f-h)\left(\lambda_{0}\right)$, где $\lambda_{0}$ - центр $U$, непрерывен на этом подпространстве и имеет норму 1 . По теореме Хана-Банаха, существует (комплексная) мера $\mu$ на $\partial U$ такая, что $\int(c f-h) d \mu=(c f-h)\left(\lambda_{0}\right)$ для всех $c, h$. Так как $\int 1 d \mu=1=\|\mu\|$, то $\mu$ - вероятностная мера. Так как $\int h d \mu=h\left(\lambda_{0}\right)$ для всех указанных $h$, то $d \mu=d \theta /(2 \pi)$, где $\theta=\arg \left(\lambda-\lambda_{0}\right), \lambda \in \partial U$. А тогда $f\left(\lambda_{0}\right)=\int_{\partial U} \frac{f d \theta}{2 \pi}$, т. е. $f$ удовлетворяет теореме о среднем для любого круга $U \Subset \mathbb{D}$. Из этого легко следует (см., например, [33]), что $f$ - комплексная гармоническая функция в $\mathbb{D}$, т. е. $f=h+\bar{g}$, где функции $h, g$ голоморфны в $\mathbb{D}$. Предположим, что $g$ не константа. Тогда функцию $g$ можно выбрать так (добавляя к $h, g$ соответствующие константы), что $g(a)=0, g^{\prime}(a) \neq 0$ в некоторой точке $a \in \mathbb{D}$. Пусть $g=u+i v$, где $u=\operatorname{Re} g$. Тогда функция $\bar{g}-g+g^{2}=u^{2}-v^{2}-2 i(v-u v)$ в окрестности $a$ не принимает вещественных отрицательных значений и, значит, $\bar{g}-g+g^{2}+\varepsilon$ не имеет там нулей, если $\varepsilon>0$. Положим $F_{\varepsilon}=h+g-g^{2}-\varepsilon$. Так как функция $f-F_{\varepsilon}=\bar{g}-g+g^{2}+\varepsilon$ не имеет нулей в окрестности $a$ при $\varepsilon>0$ и $a-$ изолированный нуль этой функции при $\varepsilon=0$, то существует круг $U \Subset \mathbb{D}$ с центром $a$ такой, что границы голоморфных графиков $S_{\varepsilon}: w=F_{\varepsilon}(\lambda), \lambda \in U, \varepsilon \in[0, \delta]$, при достаточно малом $\delta$ образуют компактное множество, не пересекающее $E$. Однако объединение графиков $S_{\varepsilon}$ по $\varepsilon \in(0, \delta)$ не содержится ни в каком компактном подмножестве в $\mathbb{D}^{2} \backslash E$. Полученное противоречие показывает, что на самом деле $g \equiv$ const, т. е. $f$ - голоморфная в $\mathbb{D}$ функция.

Теперь разберем общую ситуацию. Так как семейство $\pi: \mathbb{D}^{2} \backslash E \rightarrow \mathbb{D}$ топологически тривиальное, то $E$ есть объединение попарно непересекающихся 
непрерывных графиков (так сказать, непрерывное движение $E_{0}$ над $(\mathbb{D}, 0)$ ). Зафиксируем произвольную точку $\left(0, w_{0}\right) \in E_{0}$ и обозначим через $f$ непрерывную в $\mathbb{D}$ функцию с $f(0)=w_{0}$, график $S$ которой принадлежит $E$. Из топологической тривиальности следует, что для всякого $\varepsilon>0$ найдется окрестность $V \supset S$ в $\mathbb{D}^{2}$ такая, что $\partial V \cap E$ пусто и $V_{0} \subset\left\{\left|w-w_{0}\right|<\varepsilon\right\}$. Положим $E^{\varepsilon}=E \cap V$. Дословно повторяя первую часть доказательства и выбирая $\delta$ в конце настолько малым, что описанные там поверхности $S_{\varepsilon}$ лежат в $V$, мы получаем, что $E_{U}^{\varepsilon} \subset \widehat{E}_{U}^{\varepsilon}$ для любого круга $U \Subset \mathbb{D}$. Переходя к пределу при $\varepsilon \rightarrow 0$, получаем, что график $S: w=f(\lambda)$ тоже обладает этим свойством, т. е. $|(f-P)(\lambda)| \leqslant \max _{\partial U}|f-P|$ для всех $\lambda \in U$ и всех многочленов $P$. А тогда, как показано во второй части доказательства, функция $f$ голоморфна в $\mathbb{D}$. Так как $\left(0, w_{0}\right)$ - произвольная точка $E_{0}$, то утверждение доказано.

Теорема 5.1. Пусть $\hat{\pi}: \widehat{M} \rightarrow B$ - голоморфное семейство компактных римановых поверхностей над односвязным банаховым комплексным многообразием, E - замкнутое (возможно, пустое) подмножество $\widehat{M}$ с вполне разрывными слоями $E_{\lambda}=E \cap \widehat{M}_{\lambda}, M=\widehat{M} \backslash E u \pi=\hat{\pi} \mid M$. Предположим, что семейство $\pi: M \rightarrow B$ локально топологически тривиальное и трансверсально диск-выпуклое, а все слои $M_{\lambda}$ гиперболические. Тогда это семейство канонически униформизуемо.

ДокАЗАтЕльство. Согласно теореме 3.1 и следствию 3.1, достаточно доказать тривиализуемость $M$ голоморфным движением над окрестностью 0 для случая, когда $B=\mathbb{B}_{2}$ - единичный шар в $\mathbb{C}^{2}$.

Сначала предположим, что $E$ непусто. Так как $E_{0}$ вполне разрывно, то существуют его конечное покрытие односвязными областями $V_{j} \subset M_{0}$ с попарно непересекающимися замыканиями. По лемме 1.1 , существуют шар $U=r B$ и области $W_{j} \subset \hat{\pi}^{-1}(U)$, биголоморфно послойно эквивалентные $U \times \mathbb{D}$, такие, что $W_{j} \cap \widehat{M}_{0}=V_{j}, \bar{W}_{j} \cap \widehat{M}_{0}=\bar{V}_{j}$. Уменьшая $U$, если надо, можем считать, что множества $\partial W_{j} \cap \hat{\pi}^{-1}(U)$ не пересекают $E$.

Пусть $V$ есть окрестность $\widehat{M}_{0} \backslash \bigcup V_{j}$ в $M_{0}$, замыкание которой не пересекает $E_{0}$. Опять по лемме 1.1 , существуют шар $U^{\prime}=r^{\prime} B$ и окрестность $W$ множества $V$ в $\widehat{M}$, замыкание которой биголоморфно послойно эквивалентно $U^{\prime} \times V$, в частности, $W$ заполняется голоморфным движением $V$ над $\left(U^{\prime}, 0\right)$. Еще уменьшая $U, U^{\prime}$ можем считать, что $U=U^{\prime}, \bar{W}$ не пересекает $E$, все $\partial W_{j} \cap \hat{\pi}^{-1}(U)$ принадлежат $W$ и, значит, $\partial W \cap \hat{\pi}^{-1}(U)$ принадлежит $\bigcup W_{j}$.

Согласно лемме 5.1, множества $E \cap W_{j}$ являются объединениями попарно непересекающихся голоморфных графиков над $U$. Так как $\partial W \cap \hat{\pi}^{-1}(U)$ не пересекает $E$, то в каждом $W_{j}$ определено объединенное голоморфное движение, заполняющее как $E \cap W_{j}$, так и $\partial W \cap W_{j}$. Так как $W_{j}$ биголоморфно послойно эквивалентны $U \times \mathbb{D}$, то по теореме Берса-Ройдена (теорема 3.3 ) существует голоморфное движение множества $V_{j} \backslash V$ над $\frac{1}{3} U$, заполняющее $W_{j} \backslash W$. Объединяя эти движения в $W_{j}$ с движением, заполняющим $W$, и удаляя из него $E \cap \hat{\pi}^{-1}(U)$, получаем горизонтально голоморфную тривиализацию семейства $M$ над шаром $\frac{1}{3} U$.

Если $E$ пустое, то надо просто фиксировать какое-нибудь голоморфное сечение $\hat{\pi}$ над окрестностью $U \ni 0$, дополнить его, как описано выше, до голо- 
морфного движения, заполняющего $\hat{\pi}^{-1}\left(\frac{1}{3} U\right)$ и ничего не удалять. Теорема доказана.

ЗАмЕчАниЕ 5.1. Если поверхности $M_{\lambda}$ компактные ( $E$ пустое) и база конечномерная (ситуация теоремы Гротендика-Берса), то условие трансверсальной диск-выпуклости выполняется автоматически и доказательство, очевидно, не требует ни леммы, ни пространств Тейхмюллера; нужны только лемма 1.1, теорема Берса-Ройдена (теорема 3.3) и теорема 2.1 или 3.1.

ЗАмечание 5.2. Теорема 5.1 не охватывает семейства сфер с не более чем двумя проколами и семейства торов. Сферы и торы (без проколов) разобраны в п. 1.3. Если $\hat{\pi}: \widehat{M} \rightarrow B$ - голоморфное семейство сфер и $M:=\widehat{M} \backslash E$, где $E=$ $s(B)$ - образ непрерывного сечения, то из трансверсальной диск-выпуклости $M$ следует (как в лемме), что $s$ - голоморфное сечение, и мы получаем ситуацию теоремы 1.1 и следствия 1.2 , по которой семейство $\pi: M \rightarrow B$ тривиальное, если на $B$ разрешимы первая и вторая проблемы Кузена (односвязность $B$ здесь ни при чем, как и в случае сфер). Если же $E=s_{1}(B) \sqcup s_{2}(B)$, то сечения $s_{1}, s_{2}$ опять-таки голоморфны (при трансверсальной диск-выпуклости $M$ ). Согласно следствию $1.2,2)$, семейство $M$ тривиальное, если на $B$ разрешима вторая проблема Кузена; в этом случае послойно универсальное накрытие $\widetilde{M}$ тоже тривиальное.

Согласно лемме 5.1, условие на $E$ можно переформулировать, как в теореме Эрла.

СлЕДСТвИЕ 5.1. Пусть $\hat{\pi}: \widehat{M} \rightarrow B$ - голоморфное семейство компактнъх римановых поверхностей рода $\geqslant 2$ над односвязным банаховым комплексным многообразием, $E$ - замкнутое (возможно, пустое) подмножество $\widehat{M}$ с вполне разрьвными слоями $E_{\lambda}=E \cap \widehat{M}_{\lambda}, M=\widehat{M} \backslash E u \pi=\hat{\pi} \mid M$. Предположим, что связные компоненты множества $Е$ являются образами голоморфных сечений проекиии $\hat{\pi}$ над $B$. Тогда семейство $\pi: M \rightarrow B$ канонически униформизуемо.

В связи с этой теоремой возникает естественный вопрос: а зачем униформизовать уже униформизованное? Ведь если $\hat{f}: \widehat{\Omega} \rightarrow \widehat{M}$ - униформизация семейства $\widehat{M}$ и $M=\widehat{M} \backslash E$, как выше, то $\hat{f} \mid \widehat{\Omega} \backslash \hat{f}^{-1}(E) \rightarrow M-$ вполне приличная униформизация семейства $\pi: M \rightarrow B$, содержащая даже больше видимой информации об $M$, чем универсальная (только что слои не односвязные). Тем не менее теорема может быть полезна в задачах униформизации именно универсалъных накрывающих многообразий, которые строятся канонически (с помощью фундаментальной группы) для любого комплексного многообразия. Как, например, в следующей теореме Гриффитса [30].

Теорема 5.2. Пусть $M \subset \mathbb{C P}_{N}$ - неприводимое алгебраическое множество размерности $n, \Sigma_{0}$ - его алгебраическое подмножество и а - гладкая точка $M \backslash \Sigma_{0}$. Тогда существует алгебраическое подмножество $\Sigma \subset M$, не содержащее точку а, но содержащее $\Sigma_{0}$ и особенности $M$ и такое, что универсальная накрывающая многообразия $M \backslash \Sigma$ биголоморфно эквивалентна ограниченной стягиваемой области Рунге в $\mathbb{C}^{n}$. 
ДокАЗАТЕЛЬСтво. Докажем утверждение индукцией по $n$; при $n=1$ утверждение верное.

Можно считать, что $M_{0}:=M \backslash \Sigma_{0} \subset \mathbb{C}^{N}$. Так как $a$ - регулярная точка $M$, то существуют многочлены $p_{1}, \ldots, p_{n}$ в $\mathbb{C}^{N}$ такие, что $p_{j}(a)=0, j=1, \ldots, n$, $d p_{1} \wedge \cdots \wedge d p_{n}(a) \neq 0$ и множество $\left\{w \subset M \cap \mathbb{C}^{N}: p_{j}(w)=0, j=1, \ldots, n\right\}$ конечное. Пусть $p_{n+1}-$ многочлен, принимающий различные значения в различных точках этого множества, и $P:=\left(p_{1}, \ldots, p_{n+1}\right)$. Замыкание в $\mathbb{C}^{n+1}$ множества $P\left(M_{0}\right)$ обозначим через $M^{1}$; это неприводимое аффинное алгебраическое множество.

Заменяя $z_{j}$ на $z_{j}+\varepsilon_{j} z_{n+1}, j=1, \ldots, n$, с подходящими малыми $\varepsilon_{j} \in \mathbb{C}$, можем считать, что проекция $M^{1}$ в пространство $\mathbb{C}^{n}$ переменных $z^{\prime}=\left(z_{1}, \ldots, z_{n}\right)$ собственная и $d z_{1} \wedge \cdots \wedge d z_{n} \neq 0$ во всех точках множества $\left\{z \in M^{1}: z^{\prime}=0\right\}$. Тогда $M^{1}$ есть множество нулей многочлена Вейерштрасса

$$
Q\left(z^{\prime}, z_{n+1}\right)=z_{n+1}^{k}+\sum_{j=0}^{k-1} c_{j}\left(z^{\prime}\right) z_{n+1}^{j} .
$$

Обозначим через $\sigma$ его дискриминантное множество как многочлена от $z_{n+1}$. Тогда при каждом $\zeta^{\prime} \in \mathbb{C}^{n} \backslash \sigma$ число геометрически различных корней многочлена $Q\left(\zeta^{\prime}, z_{n+1}\right)$ равно $k$. В частности, это верно для $\zeta^{\prime}=0$.

Множество $\sigma$ или пустое, или является алгебраическим подмножеством в $\mathbb{C}^{n}$ размерности $n-1$. Сделав подходящую линейную замену координат, можем считать, что проекция множества $\sigma$ в пространство $\mathbb{C}^{n-1}$ переменных $\lambda:=$ $\left(z_{1}, \ldots, z_{n-1}\right)$ тоже собственная и что число $s$ точек множества $\sigma \cap\{\lambda=0\}$ максимально возможное (равно степени проекции $\sigma$ в $\mathbb{C}^{n-1}$ ).

Обозначим через $\sigma^{\prime}$ множество точек $\lambda \in \mathbb{C}^{n-1}$ таких, что

$$
\#\left(\sigma \cap\left\{\left(z_{1}, \ldots, z_{n-1}\right)=\lambda\right\}\right)<s ;
$$

тогда $\sigma^{\prime}$ - алгебраическое подмножество в $\mathbb{C}^{n-1}$ размерности $\leqslant n-2$. Положим

$$
\Sigma^{1}=\left\{z \in M^{1}: z^{\prime} \in \sigma \text { или }\left(z_{1}, \ldots, z_{n-1}\right) \in \sigma^{\prime}\right\}
$$

и определим $M_{\lambda}^{1}=\left\{z \in M^{1} \backslash \Sigma^{1}:\left(z_{1}, \ldots, z_{n-1}\right)=\lambda\right\}$. Так как $M^{1}$ неприводимое, то $M^{1} \backslash \Sigma^{1}$ связное, но его одномерные слои $M_{\lambda}^{1}$ хоть и гладкие, но a priori не обязательно связные.

Пусть $\lambda_{0} \in \mathbb{C}^{n-1} \backslash \sigma^{\prime}, \mathbb{B}$ - шар $\left|\lambda-\lambda_{0}\right|<r$, непересекающий $\sigma^{\prime}$, и $\pi: \mathbb{C}^{n} \rightarrow$ $\mathbb{C}^{n-1}$ - проекция $z^{\prime} \mapsto\left(z_{1}, \ldots, z_{n-1}\right)$. Тогда $\sigma \cap \pi^{-1}(\mathbb{B})$ состоит из $s$ попарно непересекающихся голоморфных графиков над $\mathbb{B}$, т. е. является пространством голоморфного движения множества $\sigma \cap\left\{\lambda=\lambda_{0}\right\}$ над $\mathbb{B}$. По теореме Берса-Ройдена (теорема 3.3), это движение продолжается до голоморфного движения $z_{n}=\phi(\lambda, \zeta): \frac{1}{3} \mathbb{B}\left(\lambda_{0}\right) \times \mathbb{C} \rightarrow \mathbb{C}$, которое определяет горизонтально голоморфную тривиализацию $\pi^{-1}\left(\frac{1}{3} \mathbb{B}\left(\lambda_{0}\right)\right)$.

При каждом фиксированном $w \in \mathbb{C} \backslash \sigma_{\lambda_{0}}$ множество точек $z \in M^{1} \backslash \Sigma^{1}$ таких, что $z^{\prime}=(\lambda, \phi(\lambda, w))$, состоит из $k$ попарно непересекающихся графиков над $\frac{1}{3} \mathbb{B}\left(\lambda_{0}\right)$ и, таким образом, семейство одномерных комплексных многообразий $M_{\lambda}^{1}$ горизонтально голоморфно тривиализуется над $\frac{1}{3} \mathbb{B}\left(\lambda_{0}\right)$.

По предположению индукции, существуют ограниченная стягиваемая область Рунге $\Omega^{\prime} \in \mathbb{C}^{n-1}$ и голоморфное накрытие $h: \Omega^{\prime} \rightarrow \mathbb{C}^{n-1} \backslash \sigma^{\prime}$. Обозначим через $M^{2}$ связную компоненту прообраза голоморфного семейства $M^{1} \backslash \Sigma^{1} \rightarrow$ 
$\mathbb{C}^{n-1} \backslash \sigma^{\prime}$ относительно $h$. Тогда $M^{2}$ есть связное голоморфное семейство одномерных комплексных многообразий над односвязной областью $\Omega^{\prime}$ и потому все слои $M_{\lambda}^{2}$ связные, т. е. являются римановыми поверхностями.

По построению, существует голоморфное послойное накрытие $H: M^{2} \rightarrow M^{1}$. Поэтому, по доказанному, семейство $M^{2} \rightarrow \Omega^{\prime}$ локально тривиализуется голоморфными движениями, и, следовательно, это семейство канонически униформизуется, т. е. существует область $\Omega \subset \Omega^{\prime} \times \mathbb{C}$ с односвязными слоями и также голоморфное послойное накрытие $f: \Omega \rightarrow M^{2}$. При этом дополнение к $\bar{\Omega}$ в $\Omega^{\prime} \times \mathbb{C}$ биголоморфно послойно эквивалентно $\Omega^{\prime} \times(\mathbb{C} \backslash \overline{\mathbb{D}})$ и $\Omega_{0}=\mathbb{D}$. Из этого очевидно следует, что область $\Omega$ стягиваемая и полиномиально выпуклая (т. е. является областью Рунге).

Теперь положим $\Sigma=\left\{w \in \mathbb{C}^{N}: P(w) \in \Sigma^{1}\right\}$. Тогда $M \backslash \Sigma=\{w: P(w) \in$ $\left.M^{1} \backslash \Sigma^{1}\right\}$, отображение $P: M \backslash \Sigma \rightarrow M^{1} \backslash \Sigma^{1}$ биголоморфное, а

$$
\Omega \ni Z \mapsto f(Z) \mapsto H(f(Z)) \mapsto P^{-1}(H(f(Z))) \in M \backslash \Sigma
$$

является универсальным накрытием многообразия $M \backslash \Sigma \ni a$. Теорема доказана.

ЗАмЕчАниЕ 5.3. Из доказательства видна структура универсальной накрывающей $\Omega$. Это область в $\Omega^{\prime} \times \mathbb{C} \subset \mathbb{C}^{n}, \Omega_{0}=\mathbb{D}$, ее граница покрывается однопараметрическим семейством попарно непересекающихся голоморфных графиков над $\Omega^{\prime}$, а дополнение к замыканию $\left(\Omega^{\prime} \times \mathbb{C}\right) \backslash \bar{\Omega}$ биголоморфно послойно эквивалентно $\Omega^{\prime} \times(\mathbb{C} \backslash \overline{\mathbb{D}})$. Область $\Omega^{\prime}$ аналогичным образом устроена над своей проекцией в $\mathbb{C}^{n-2}$ и т. д. В целом $\Omega$ удобно представлять как "квазиголоморфный поликруг" $\left(\mathbb{D}^{n}, J\right)$ с нестандартной комплексной структурой $J$, базовые $(1,0)$-формы которой имеют вид $d z_{1}, d z_{2}-\mu_{2} d \bar{z}_{2}-a_{21} d \bar{z}_{1}, \ldots, d z_{n}-\mu_{n} d \bar{z}_{n}-$ $a_{n 1} d \bar{z}_{1}-\cdots-a_{n n-1} d z_{n-1}$ с гладкими коэффициентами и $\left|\mu_{j}\right|<1$.

5.2. Семейства с диск-плоскими границами. Голоморфное семейство $\pi: M \rightarrow B$ римановых поверхностей назовем трансверсально диск-вогнутым, если для каждой точки $\lambda^{\prime} \in B$ существует окрестность $U^{\prime} \ni \lambda^{\prime}$ со следующим свойством: для любого вложенного голоморфного диска $h: \overline{\mathbb{D}} \rightarrow U^{\prime}$, любого компакта $K \subset \pi^{-1}(h(\mathbb{D}))$ и любого $r \in(0,1)$ найдется компакт $K^{\prime} \subset \pi^{-1}(h(\overline{\mathbb{D}}))$ такой, что если $f: \mathbb{D} \rightarrow M$ - голоморфный диск, $\pi \circ f=h$ и $f(\mathbb{D} \backslash r \mathbb{D})$ не пересекает $K^{\prime}$, то $f(\mathbb{D})$ не пересекает $K$.

У автора нет уверенности в том, что для трансверсальной диск-вогнутости справедлив аналог леммы 1.3, т. е. что это свойство автоматически распространяется на семейство $\widetilde{M} \rightarrow B$, где $\widetilde{M} \rightarrow M$ - голоморфное послойно универсальное накрытие. Однако это верно при дополнительных условиях.

Будем говорить, что семейство $\pi: M \rightarrow B$ имеет диск-плоскую границу, если оно одновременно трансверсально диск-выпуклое и трансверсально диск-вогнутое.

ТеОрема 5.3. Топологически тривиальное голоморфное семейство $\pi: M \rightarrow$ $\mathbb{D}$ гиперболических римановых поверхностей с диск-плоской границей канонически униформизуемо.

ДоказАтельство. Пусть $f_{0}: \mathbb{D} \rightarrow M_{0}$ - универсальное накрытие. Так как семейство $M$ топологически тривиальное, то оно гладко тривиальное (см. [46]) 
и потому универсальной накрывающей $M$ является комплексное многообразие $(\mathbb{D} \times \mathbb{D}, J)$, где $J$ - согласованная комплексная структура с определяющей формой $d w-\mu d \bar{w}-a d \bar{\lambda}$ с гладкими коэффициентами и $|\mu|<1$ (см. 4.1). Универсальное накрытие $M$, равное $f_{0}$ над $M_{0}$, обозначим через $f$.

Пусть $\lambda_{0} \in B$ и $U=\left\{\left|\lambda-\lambda_{0}\right|<r\right\} \Subset \mathbb{D}$ - круг из определения диск-плоской границы, над замыканием которого есть голоморфное сечение $s_{0}$ проекции $\pi$. Покажем, что семейство $\pi_{1}^{-1}(U)$ тривиализуется $J$-голоморфным движением. Сделав подходящую гладкую замену вертикальной координаты $w$ над окрестностью $\bar{U}$, можем считать, что функция $w$ постоянна на каждом диске из множества $f^{-1}(s(U))$ и $w=0$ на одном из этих дисков. Тогда $a(\lambda, 0) \equiv 0$ в $U$. Так как структура $J$ трансверсально диск-выпуклая по лемме 1.3 , то структура $J$ - такая, как в замечании 4.1 к теореме 4.1 , и потому над $U$ существует голоморфное движение $w=\phi(\lambda, z), z \in \mathscr{D}_{0}$, непрерывное над $\bar{U}$, заполняющее $\partial U \times \mathbb{D}$ и некоторую область $\mathscr{D}$ с односвязными слоями $\mathscr{D} \lambda$. По конструкции (см. теорему 4.1), область $\mathscr{D}$ содержит также все множество $f^{-1}\left(s_{0}(U)\right)$.

Покажем, что область $\mathscr{D}$ инвариантна относительно накрывающей группы $G$ универсального накрытия $f:\left(\mathbb{D}^{2}, J\right) \rightarrow M$. Пусть $s_{1}: w=\phi\left(\lambda, z_{1}\right)$ и $g \in G$. Соединим $s_{1}$ и $s_{0}$ непрерывным семейством $J$-голоморфных дисков $s_{t}: w=$ $\phi\left(\lambda, z_{t}\right)$. Обозначим через $E$ множество таких $t \in[0,1]$, что $g\left(s_{t}\right) \in \mathscr{D}$. Так как $\mathscr{D}$ - область и $\phi\left(\cdot, z_{t}\right)$ непрерывны в $\bar{U}$, то множество $E$ открыто в $[0,1]$. Согласно дополнению к теореме 4.1, для $t \in E$ справедлива оценка $\left|w \circ s_{t}(\lambda)\right| \leqslant$ $\max \{|\phi(\lambda, z)|:|z| \leqslant R\}$, где $R=\max _{\partial U}\left|w \circ s_{t}\right|$. Из нее очевидно следует, что множество $E$ замкнуто и потому совпадает с $[0,1]$.

Так как многообразие $(\mathscr{D}, J)$ тривиализовано голоморфным движением, то по лемме 2.6 существует послойное биголоморфное отображение $F: \Omega \rightarrow(\mathscr{D}, J)$ с заданным универсальным накрытием $F\left(\lambda_{0}, \cdot\right): \mathbb{D} \rightarrow \mathscr{D}_{0}$, где $\Omega$ - область в $U \times \mathbb{C}$ (каноническая униформизация). Прообраз группы $G$ относительно $F$ обозначим $\tilde{G}$. Тогда (см. п. 3.1) элементы $\tilde{G}$ продолжаются до послойных биголоморфизмов $U \times \widehat{\mathbb{C}}$, дробно-линейных по вертикальной переменной. Так как $\Omega$ инвариантна относительно $\tilde{G}$, то голоморфное движение $\partial \mathbb{D}$ над $\left(U, \lambda_{0}\right)$, заполняющее границу $\partial \Omega$ в $U \times \mathbb{C}$, тоже инвариантно относительно $\tilde{G}$. По теореме Слодковского [57] (см. п. 3.3, следствие 4.7 и $\lambda$-лемму 2.4), существует голоморфное движение, заполняющее $\Omega$ и $\tilde{G}$-инвариантное. Его прообраз $\psi: U \times \mathscr{D}_{0} \rightarrow \mathbb{D}$ относительно $F$ является $G$-инвариантным и заполняет всю $\mathscr{D}$.

Положим $\mathscr{D}_{0}^{\prime}:=f\left(0 \times \mathscr{D}_{0}\right) \subset M_{0}$. Тогда существует однозначно определенное голоморфное движение $\psi^{\prime}: U \times \mathscr{D}_{0}^{\prime} \rightarrow \pi^{-1}(U)$, непрерывное в $\bar{U} \times \mathscr{D}_{0}^{\prime}$, заполняющее область $f(\mathscr{D})=: \mathscr{D}^{\prime}$ и такое, что $f(\lambda, \psi(\lambda, z)) \equiv \psi^{\prime} \circ f(\lambda, z)$ в $U \times \mathscr{D}_{0}$ (проекция движения $\psi$ в $M$ ).

Пусть $a \in M_{\lambda}, \lambda \in U,-$ предельная точка для $\mathscr{D}_{\lambda}^{\prime}, a_{\nu} \in \mathscr{D}_{\lambda}, a_{\nu} \rightarrow a$, $K$ - компактная окрестность $a$ в $M_{\lambda}$ и $s_{\nu}=\psi^{\prime}\left(\cdot, b_{\nu}\right)$ таковы, что $a_{\nu}=s_{\nu}(\lambda)$. Так как области $\mathscr{D}_{\lambda}$ стремятся к $\mathbb{D}$, заполняя каждый диск $\theta \mathbb{D}, \theta<1$, при $\left|\lambda-\lambda_{0}\right| \rightarrow r$, то для любого компакта $K^{\prime} \subset \pi^{-1}(U)$ найдется $r^{\prime} \in(0, r)$ такое, что $K^{\prime} \cap\left\{r^{\prime} \leqslant\left|\lambda-\lambda_{0}\right|<r\right\}$ содержится в $\mathscr{D}^{\prime}$. Так как $s_{\nu}$ непрерывны в $\bar{U}$ и последовательность образов сечений $s_{\nu}$ не имеет предельных точек в $\mathscr{D}^{\prime}$, то для любого компакта $K^{\prime} \subset \pi^{-1}(\bar{U})$ найдутся $r^{\prime} \in(0, r)$ и номер $\nu^{\prime}$ такой, что кольцо $s_{\nu}\left(\left\{r^{\prime} \leqslant\left|\lambda-\lambda_{0}\right| \leqslant r\right\}\right)$ не пересекает $K^{\prime}$ при $\nu>\nu^{\prime}$. Это противоречие с трансверсальной диск-вогнутостью семейства $M$ показывает, что на самом деле 
$\mathscr{D}=U \times \mathbb{D}$ и, значит, семейство $M$ горизонтально голоморфно тривиализуется над $U$. Так как это верно для любого малого круга $U \Subset \mathbb{D}$, то по теореме 2.1 семейство $\pi: M \rightarrow B$ канонически униформизуемо. Теорема доказана.

Автору не известно, справедливо ли утверждение теоремы для семейств с диск-плоскими границами над произвольной базой $B$, так как теорема 3.2 о редукции базы доказана лишь для семейств римановых поверхностей без края. Конечно, с каждым семейством поверхностей $M_{\lambda}$ с краем можно связать семейство дублей Шоттки $\widehat{M}_{\lambda}, \lambda \in B$, уже не имеющих края, однако определение на нем комплексной структуры, превращающей это семейство в голоморфное, совсем не очевидно. Впрочем, если $B=\mathbb{D}$, то это следует из доказанной теоремы. В самом деле, если $f_{0}: \mathbb{D} \rightarrow M_{0}$ - универсальное накрытие с накрывающей группой $\Gamma$ и $f: \Omega \rightarrow M, f(0, \cdot)=f_{0},-$ каноническая униформизация, то в $\mathbb{D} \times \widehat{\mathbb{C}}$ действует группа $G$ голоморфных послойных автоморфизмов, относительно которой $\Omega$, а следовательно и $(\mathbb{D} \times \widehat{\mathbb{C}}) \backslash \Omega$, инвариантны. Сужение этой группы на слой $\lambda \times \widehat{\mathbb{C}}$ обозначим $G_{\lambda}$. Пусть $E:=\bigsqcup E_{\lambda}-$ предельное множество группы $G$. Так как все $M_{\lambda}$ имеют непустой край, то множества $\widehat{\mathbb{C}} \backslash E_{\lambda}$ связные. Фактор области $(\mathbb{D} \times \widehat{\mathbb{C}}) \backslash E$ по действию группы $G$ является комплексным многообразием, расслоенным над $\mathbb{D}$ в семейство римановых поверхностей $\widehat{M}_{\lambda}:=\left(\widehat{\mathbb{C}} \backslash E_{\lambda}\right) / G_{\lambda}$, каждая из которых является дублем Шоттки соответствующей $M_{\lambda}$. Так как $\widehat{\mathbb{C}} \backslash E_{\lambda} \rightarrow \widehat{M}_{\lambda}$ - накрытия и $E_{\lambda}$ вполне разрывные, то $\widehat{M}_{\lambda}$ не имеют края.

Комбинируя теорему 5.2 с теоремой 3.2 , получаем следующее утверждение.

СледСтвиЕ 5.2. Топологически локалъно тривиальное голоморфное семейство $\pi: M \rightarrow B$ с диск-плоской гранищей гиперболических римановых поверхностей без края над произвольным односвязным комплексным банаховым многообразием канонически униформизуемо.

5.3. Плоскости. Теорема Нишино. Голоморфные семейства $\pi: M \rightarrow B$ римановых поверхностей, конформно эквивалентных плоскости $\mathbb{C}$, выпадают из разобранной выше схемы, основанной на теории пространств Тейхмюллера, и требуют самостоятельного изучения. Первое ощущение, что такие семейства являются локально голоморфно тривиальными (как семейства сфер), опровергается простыми примерами (см. пример 4.1). Более того, пример 1.2 показывает, что в общем такие семейства не униформизуются даже областями B $B \times \widehat{\mathbb{C}}$.

Вместе с тем, Т. Нишино доказал, что если $B=\mathbb{D}$, все $M_{\lambda}$ конформно эквивалентны $\mathbb{C}$, проекция $\pi$ допускает глобальное голоморфное сечение и $M$ многообразие Штейна, то семейство $\pi: M \rightarrow \mathbb{D}$ является голоморфно тривиальным, т.е. биголоморфно эквивалентным семейству $\mathbb{D} \times \mathbb{C} \rightarrow \mathbb{D}$ (см. [49; c. 253]).

Здесь мы докажем аналогичное утверждение (для общей базы $B$ ) при более слабых условиях на $M$.

Теорема 5.4. Пусть $\pi: M \rightarrow B$ - голоморфное трансверсалъно диск-въпуклое семейство римановых поверхностей над комплексным банаховым многообразием, со слоями $M_{\lambda}$, конформно эквивалентными плоскости $\mathbb{C}$. Тогда это семейство локально голоморфно тривиальное и оно глобально голоморфно тривиальное, если выполнено одно из следующих условий: 
1) на В разрешимы первая и вторая проблемы Кузена;

2) $\pi$ допускает глобальное голоморфное сечение и на В разрешима вторая проблема Кузена;

3) $\pi$ допускает два глобальных голоморфных сечения с непересекающимися образами.

ДокАЗАТЕЛЬСтво. ШАГ 1. Вертикальная координата. Предположим, что $\pi$ допускает два глобальных голоморфных сечения $s_{0}, s_{1}$ с непересекающимися образами. Тогда для каждого $\lambda \in B$ однозначно определено однолистное голоморфное отображение $W_{\lambda}: M_{\lambda} \rightarrow \mathbb{C}$ такое, что $W_{\lambda} \circ s_{0}=0, W_{\lambda} \circ s_{1}=1$. Наша задача - показать, что функция $W$ на $M$, равная $W_{\lambda}$ на $M_{\lambda}, \lambda \in B$, голоморфна. Это совсем неочевидно, даже непрерывность $W$ надо доказывать (cp. с [49; с. 225-228]), чем мы сейчас и займемся. Нам достаточно доказать это при дополнительном предположении, что многообразие $B$ конечномерное.

Фиксируем произвольную точку $\lambda_{0} \in B$. По теореме Сью [55], существует штейнова окрестность $U \supset M_{\lambda_{0}}$ на $M$. Уменьшая ее, можно считать, что существует голоморфная в $U$ функция $z$ такая, что $z=W$ на $M_{\lambda_{0}}$ и отображение $p \mapsto(\pi(p), z(p))$ биголоморфно переводит $U$ в некоторую область $U^{\prime} \subset B \times \mathbb{C}$. Заменяя $z$ на $\left(z-z \circ s_{0}\right) /\left(z \circ s_{1}-z \circ s_{0}\right)$, можем считать, что $z \circ s_{0} \equiv 0$ и $z \circ s_{1} \equiv 1$ (там, где это определено).

Образ функции $W$ при этом отображении обозначим через $W(\lambda, z)$. Так как $U^{\prime} \supset \lambda_{0} \times \mathbb{C}$, то существует функция $R(\lambda)>0$ такая, что $R(\lambda) \rightarrow \infty$ при $\lambda \rightarrow \lambda_{0}$ и функция $W(\lambda, \cdot)$ определена, голоморфна и однолистна на $R(\lambda) \mathbb{D}$, причем $W(\lambda, 0) \equiv 0, W(\lambda, 1) \equiv 1$. Тогда $W(\lambda, \cdot)$ отображает проколотые круги $\{z:|z|<R(\lambda), z \neq 0,1\}$ в гиперболическую область $\mathbb{C} \backslash\{0,1\}$. По лемме Шварца для метрики Кобаяши (п. 2.1) и принципу максимума, для всякого $R>0$ найдется окрестность $V \ni \lambda_{0}$ в $B$ такая, что семейство таких функций $W(\lambda, \cdot)$, $\lambda \in V$, равностепенно непрерывно в круге $|z|<R$. Поэтому из произвольной последовательности $W\left(\lambda_{j}, \cdot\right), \lambda_{j} \rightarrow \lambda_{0}$, можно выделить подпоследовательность, равномерно сходящуюся внутри $\mathbb{C}$ к некоторой голоморфной функции. Эта предельная функция однолистна, равна 0, 1 в точках 0, 1 соответственно и потому совпадает с $W_{\lambda_{0}}$. Так как это верно для любой последовательности $\lambda_{j} \rightarrow \lambda_{0}$, то $W(\lambda, \cdot) \rightarrow W\left(\lambda_{0}, \cdot\right)$ равномерно внутри $\mathbb{C}$ при $\lambda \rightarrow \lambda_{0}$.

ШАГ 2. Одномерная база. Теперь докажем голоморфную тривиальность в одномерной ситуации, когда $B$ - круговая окрестность $\overline{\mathbb{D}}$ в $\mathbb{C}$, предположив, что $\pi$ допускает два глобальных голоморфных сечения $s_{0}, s_{1}$ с непересекающимися образами и что комплексная структура на $M$ диск-выпуклая над $\mathbb{D}$.

Так как все $M_{\lambda}$ диффеоморфны $\mathbb{R}^{2}$, то существует диффеоморфизм $M \rightarrow$ $B \times \mathbb{C}$, сохраняющий проекции и ориентации (см. [46]). Таким образом, $M$ биголоморфно послойно эквивалентно многообразию $(B \times \mathbb{C}, J)$ с комплексной $(=$ интегрируемой почти комплексной) структурой $J$, относительно которой проекция $(\lambda, w) \mapsto \lambda$, т. е. функция $\lambda$, голоморфна. Определяющая форма структуры $J$ имеет вид $d w-\mu d \bar{w}-a d \bar{\lambda}$; единственное, что мы знаем о $\mu$ и $a,-$ это что они гладкие и $|\mu|<1$, но отнюдь не обязательно равномерно. Указанный диффеоморфизм можно подправить так, чтобы сечения $s_{0}, s_{1}$ переходили соответственно в $w=0,1$. Тогда $a(\lambda, 0) \equiv a(\lambda, 1) \equiv 1$, что мы и предполагаем в дальнейшем. Образ функции $W$ из шага 1 на $B \times \mathbb{C}_{w}$ обозначим через $W(\lambda, z)$. 
Так как $W$ голоморфна на $M_{\lambda}$ при каждом $\lambda \in B$, то $W(\lambda, \cdot)$ удовлетворяет уравнению Бельтрами $W_{\bar{z}}=\mu(\lambda, \cdot) W_{z}$, вообще говоря вырожденному, так как $\|\mu(\lambda, \cdot)\|_{\infty}$ может равняться 1 . Тем не менее $W(\lambda, w) \rightarrow \infty$ при $w \rightarrow \infty$ локально равномерно по $\lambda$, так как функция $W$ на $M$ непрерывна - по доказанному выше.

Структура $J$ удовлетворяет условиям теоремы 4.1, согласно которой существует $J$-голоморфное движение $\phi: \overline{\mathbb{D}} \times \mathbb{C} \rightarrow \mathbb{C}$, заполняющее $\partial \mathbb{D} \times \mathbb{C}$ и некоторую область $\mathscr{D}$ над $\mathbb{D}$ с односвязными слоями $\mathscr{D}_{\lambda}$. Вертикальную параметризацию движения $\phi$ удобнее перенести в $\lambda=0$, т. е. заменить $\phi(\lambda, \cdot)$ на $\phi_{0}(\lambda, \cdot):=$ $\phi(\lambda, \cdot) \circ \phi(0, \cdot)^{-1}$. Тогда $\phi_{0}: \overline{\mathbb{D}} \times \mathscr{D}_{0} \rightarrow \mathbb{C}$ - обычное $J$-голоморфное движение $\mathscr{D}_{0}$ над $(\mathbb{D}, 0)$, непрерывное в $\overline{\mathbb{D}} \times \mathbb{C}$. По построению и теореме единственности (лемма 4.2$), \phi_{0}(\lambda, 0) \equiv 0$ и $\phi_{0}(\lambda, 1) \equiv 1$.

Покажем, что $\mathscr{D}_{0}=\mathbb{C}$. Если это не так, то $\mathscr{D}_{0}$ конформно эквивалентно кругу $\mathbb{D}$ и, предположив это, мы фиксируем конформное отображение $f_{0}: \mathbb{D} \rightarrow \mathscr{D}_{0}$. Так как структура $J$ интегрируема (комплексна), то ей соответствует характеристика $\nu(\lambda, z)$ движения $\phi_{0}$, которая по лемме 2.5 голоморфна по $\lambda \in \mathbb{D}$ (как отображение $\lambda \mapsto \nu(\lambda, \cdot) \in L^{\infty}(\mathbb{D})$; см. п. 2.2). Так как $|\nu|<1$ и $\nu(0, \cdot)=0$, то $\|\nu(\lambda, \cdot)\|_{\infty} \leqslant \lambda$ и, следовательно, все отображения $\phi_{0}(\lambda, \cdot): \mathscr{D}_{0} \rightarrow \mathscr{D}_{\lambda}$ являются $J$-квазиконформными, т. е. квазиконформными относительно координаты $W(\lambda, \cdot)$.

Пусть $\psi: \mathbb{D} \times \mathbb{C} \rightarrow \mathbb{C}$ - каноническое накрытие движения $\phi_{0}$ (см. 2.2), т. е. $\psi(\lambda, \cdot): \mathbb{C} \rightarrow \mathbb{C}-$ квазиконформные отображения с коэффициентами Бельтрами $\nu(\lambda, \cdot)$, сосредоточенными в $\mathbb{D}$, нормированные условием $\psi(\lambda, z)=z+o(1)$ в окрестности $\infty$. Так как $\psi(\lambda, \cdot)$ голоморфно и однолистно в $\mathbb{C} \backslash \overline{\mathbb{D}}$, то по теореме Кёбе образ круга $\overline{\mathbb{D}}$ при таком отображении (с произвольной $\lambda \in \mathbb{D})$ лежит в круге $4 \mathbb{D}$.

Положим $F(\lambda, \cdot)=\psi(\lambda, \cdot) \circ f_{0}^{-1} \circ \phi_{0}(\lambda, \cdot)^{-1}$. Тогда функция $F$ определена и $J$-голоморфна в области $\mathscr{D}, F(0, \cdot)=f_{0}^{-1}$ и для всякого $\lambda \in \mathbb{D}$ функция $F(\lambda, \cdot)$ однолистна в $\mathscr{D}_{\lambda}$, причем $F\left(\lambda, \mathscr{D}_{\lambda}\right)=\psi(\lambda, \mathbb{D})$.

Функция $F(\lambda, 1)-F(\lambda, 0)$ голоморфна, ограничена (ее модуль не превосходит 8) и не имеет нулей в $\mathbb{D}$. Поэтому у нее есть ненулевые угловые предельные значения почти в каждой точке из $\partial \mathbb{D}$. Пусть $\zeta \in \partial \mathbb{D}$ - такая точка. Положим $G(\lambda, \cdot):=(F(\lambda, \cdot)-F(\lambda, 0)) /(F(\lambda, 1)-F(\lambda, 0))$ и определим функцию $g(\lambda, W)$ условием $g(\lambda, W(\lambda, w))=G(\lambda, z(\lambda, w))$. Тогда $g$ голоморфна по $W$ уже в обычном смысле (хотя голоморфность по $\lambda$ - под вопросом), $g(\lambda, 0) \equiv 0$, $g(\lambda, 1) \equiv 1$ и $|g(\lambda, W)| \leqslant M$ с некоторой константой $M$ для всех $\lambda=t \zeta, t \in(0,1)$, и $W \in W\left(\lambda, \mathscr{D}_{\lambda}\right)$. Но движение $\phi_{0}$ непрерывно в $\overline{\mathbb{D}} \times \mathbb{C}$ и заполняет все граничное множество $\partial \mathbb{D} \times \mathbb{C}$. Поэтому существует $\theta<1$ такое, что $W\left(\lambda, \mathscr{D}_{\lambda}\right)$ содержит круг $2 M \mathbb{D}$ при $|\lambda|>\theta$. Итак, если $t>\theta$, то $g(t \zeta, \cdot) \mid 2 M \mathbb{D} \rightarrow M \mathbb{D}$ и, значит, $|g(t \zeta, W)| \leqslant|W| / 2$ по лемме Шварца, в частности, $|g(t \zeta, 1)| \leqslant 1 / 2$. Полученное противоречие $($ с $g(\lambda, 1) \equiv 1)$ показывает, что $\mathscr{D}_{0}=\mathbb{C}$. А тогда и все $\mathscr{D}_{\lambda}$ равны $\mathbb{C}$, поскольку отображения $\phi_{0}(\lambda, \cdot) J$-квазиконформные.

Таким образом, функция $F$ определена уже всюду на $\mathbb{D} \times \mathbb{C}$ и отображение $(\lambda, w) \mapsto(\lambda, F(\lambda, w))$ биголоморфно относительно структуры $J$ в $\mathbb{D} \times \mathbb{C}$ и обычной комплексной структуры в $\mathbb{C}^{2}$. 
ШАГ 3. Два глобальных сечения. Предположим теперь, что $B$ произвольное, но есть два сечения $s_{0}, s_{1}$, как выше. Фиксируем произвольную точку $\lambda_{0} \in B$. Пусть $U \ni \lambda_{0}$ - окрестность из определения трансверсальной диск-выпуклости и $h: \overline{\mathbb{D}} \rightarrow U-$ вложенный голоморфный диск. Согласно шагу 1 , существует биголоморфное отображение $\pi^{-1}(h(\mathbb{D})) \rightarrow \mathbb{D} \times \mathbb{C}$ вида $p \mapsto(\pi(p), H(p)), H \circ s_{0} \equiv 0, H \circ s_{1} \equiv 1$. Но тогда $H \mid M_{\lambda}=W_{\lambda}$ для всех $\lambda \in h(\mathbb{D})$, т. е. функция $W$ из шага 1 голоморфна в $\pi^{-1}(h(\mathbb{D}))$. Так как она локально ограничена в $B \times \mathbb{C}$, то отсюда следует голоморфность $W$ в произвольном координатном шаре в $U \times \mathbb{C}$ и, значит, отображение $M \ni p \mapsto(\pi(p), W(p)) \in B \times \mathbb{C}$ биголоморфно.

ШАГ 4. Проблемы Кузена. Локально голоморфные сечения $\pi$ существуют в любом количестве. Поэтому, согласно шагу 3 , семейство $\pi: M \rightarrow B$ локально голоморфно тривиализуется, т. е. для каждого $\alpha \in B$ существуют окрестность $V_{\alpha} \ni \alpha$ (координатный шар) и голоморфная в $U_{\alpha}:=\pi^{-1}\left(V_{\alpha}\right)$ функция $W_{\alpha}$, однолистная в слоях $M_{\lambda}, \lambda \in V_{\alpha}$. Если $\pi$ допускает глобальное голоморфное сечение $s$, то все $W_{\alpha}$ можно выбирать так, что $W_{\alpha} \circ s \equiv 0$. А тогда в $U_{\alpha} \cap U_{\beta}$ имеем $W_{\beta}=c_{\beta \alpha} W_{\alpha}$ с голоморфной функцией $c_{\beta \alpha}$, постоянной на слоях $M_{\lambda}$. Эти $c_{\beta \alpha}$ образуют мультипликативный 1-коцикл на $B$ (соответствующий покрытию $\left.\left\{V_{\alpha}\right\}\right)$. Если на $B$ разрешима вторая проблема Кузена, то существуют голоморфные в $U_{\alpha}$ функции $c_{\alpha}$, постоянные на слоях $M_{\lambda}$ и нигде не равные нулю, такие, что $c_{\beta \alpha}=c_{\alpha} / c_{\beta}$ в $U_{\alpha} \cap U_{\beta}$. А тогда, полагая $W=c_{\alpha} W_{\alpha}$ в $U_{\alpha}$, $\alpha \in B$, получаем глобальную голоморфную функцию $W$ на $M$, однолистную в слоях, а с нею и биголоморфизм $M \rightarrow B \times \mathbb{C}$.

Наконец, если на $B$ разрешимы и вторая, и первая проблемы Кузена, то просто повторим доказательство следствия 1.2 (заменяя в нем $z_{\alpha}$ на $W_{\alpha}$ ). Теорема 5.4 доказана.

СлеДСтвиЕ 5.3. Голоморфное семейство $\pi: M \rightarrow B$ со слоями, конформно эквивалентными $\mathbb{C}$, допускает локальную голоморфную тривиализацию тогда и только тогда, когда оно продолжается до голоморфного семейства сфер, m.е. существуют голоморфное семейство $\hat{\pi}: \widehat{M} \rightarrow B$ со слоями, конформно эквивалентными сфере Римана $\widehat{\mathbb{C}}$, и биголоморфное послойное отображсние $M \rightarrow \widehat{M} \backslash s(B)$, где $s$ - некоторое глобальное голоморфное сечение проекиии $\hat{\pi}$.

ДоказАтельство. Положим $\widehat{M}_{\lambda}=M_{\lambda} \sqcup \infty, \widehat{M}=\bigsqcup_{\lambda} \widehat{M}_{\lambda}$ и $\hat{\pi}: \widehat{M}_{\lambda} \rightarrow \lambda$. Локальные "вертикальные" координаты $W^{\alpha}$ из шага 3 очевидно продолжаются до однолистных мероморфных функций в каждом слое $\widehat{M}_{\lambda}, W(\infty):=\infty$. Функции $W^{\alpha}$, которые по определению считаем мероморфными в $U_{\alpha}$, вместе с функциями вида $h \circ \hat{\pi}$, где $h$ голоморфна в $V_{\alpha}$, определяют на $\widehat{M}$ комплексную структуру (очевидно, не зависящую от индексов $\alpha$ ). Таким образом определено голоморфное семейство $\hat{\pi}: \widehat{M} \rightarrow B$, включающее, как описано, исходное семейство плоскостей.

Обратное утверждение очевидно ввиду теоремы 1.1. Следствие доказано.

Интересно, можно ли условие типа псевдовыпуклости в теореме Нишино заменить условием другого типа, обеспечивающим голоморфность особенностей 
при наличии в них комплексной структуры, например условием плюриполярности (существуют замкнутое множество $X \subset M$ с компактными слоями и функция $u$, плюрисубгармоническая в $M \backslash X$ и такая, что $u\left(p_{j}\right) \rightarrow-\infty$ для любой последовательности $\left\{p_{j}\right\} \subset M$, не имеющей предельных точек в $\left.M\right)$. По этому поводу см. [53].

\section{6. Об устранимых особенностях комплексных структур}

6.1. Семейства проколотых дисков. Согласно следствию 5.3, теорему Нишино можно рассматривать как теорему о продолжении комплексной структуры с многообразия $M$, состоящего из плоскостей, на объемлющее его многообразие $\widehat{M}$, слоями которого являются сферы. В общем случае $\widehat{M}-$ лишь гладкое многообразие, но псевдовыпуклость (трансверсальная диск-выпуклость) семейства $M$ позволяет наделить $\widehat{M}$ комплексной структурой, совпадающей с таковой на $M$. При этом множество "бесконечно удаленных точек" $\widehat{M}$ оказывается голоморфным сечением проекции семейства $\widehat{M}$ на базу, что видно из доказательства теоремы 5.4 (вертикальная мероморфная координата $z$ на нем равна $\infty$ ), а также следует из классической теоремы Гартогса об аналитичности особенностей (см. [51]) после того, как комплексная структура продолжена на $\widehat{M}$.

Теорема Гартогса утверждает, что если $S: w=f(\lambda)$ - непрерывный график над $B,|f|<1$ и область $(B \times \mathbb{D}) \backslash S$ псевдовыпуклая, то функиия $f$ голоморфна. В этой ситуации область $(B \times \mathbb{D}) \backslash S$ над $B$ расслаивается в семейство проколотых кругов и теорема утверждает, что проколы голоморфно зависят от параметра $\lambda \in B$. Объемлющее многообразие $B \times \mathbb{D}$ здесь имеет комплексную структуру всюду, включая проколы.

В связи с теоремой Нишино естественно возникает вопрос о справедливости аналогичного утверждения на комплексных многообразиях, состоящих из проколотых дисков: можно ли и структуру продолжить и получить аналитичность особенностей? Положительный ответ на этот вопрос при $B=\mathbb{D}$ фактически следует из теоремы Нишино, а в общем случае нужны дополнительные соображения, как в теореме 5.4. Следующая теорема представляет локальный вариант теоремы Нишино.

Теорема 6.1. Пусть $\pi: M \rightarrow B$ - голоморфное семейство римановых поверхностей $M_{\lambda}$, конформно эквивалентных проколотому кругу. Если многообразие $B$ конечномерное и семейство $M$ трансверсально диск-выпуклое, то существуют комплексное многообразие $\widehat{M}$, голоморфная проекция $\hat{\pi}: \widehat{M} \rightarrow B$ со слоями, конформно эквивалентными кругу, и голоморфное послойное вложение $F: M \rightarrow \widehat{M}$ такие, что $\widehat{M} \backslash F(M)$ есть образ голоморфного сечения проекиии $\hat{\pi}$ над $B$.

ДокАЗАТЕльство. Предположим сначала, что семейство $M$ топологически тривиальное. Тогда существует послойный диффеоморфизм $M$ на $B \times(\mathbb{D} \backslash 0)$ (см. [46]). Образ комплексной структуры $M$ при этом отображении обозначим через $J$; таким образом, многообразие $M$ биголоморфно эквивалентно $(B \times$ $(\mathbb{D} \backslash 0), J)$. При этом гладкую вертикальную координату $w$ можно выбрать так, 
что для фиксированной $\lambda_{0}$ функция $w$ голоморфна на $\lambda_{0} \times(\mathbb{D} \backslash 0)$ относительно структуры $J$.

По теореме Сью [55], существует штейнова окрестность $\mathscr{U}$ проколотого диска $\lambda_{0} \times(\mathbb{D} \backslash 0)$ в $(B \times(\mathbb{D} \backslash 0), J)$. Уменьшая $\mathscr{U}$, если надо, можем считать, что в $\mathscr{U}$ существует $J$-голоморфная функция $f$ такая, что $f\left(\lambda_{0}, w\right)=w$ и $d f \neq 0$ на слоях $(\lambda \times \mathbb{D}) \cap \mathscr{U}$. Пусть $U \ni \lambda_{0}-$ координатная окрестность, настолько малая, что уровни $\{f(\lambda, w)=c\}$ с $\lambda \in U$ и $1 / 4<|c|<3 / 4$ являются графиками над $U$ и их объединение содержит множество $U \times\{1 / 3 \leqslant|w| \leqslant 2 / 3\}$. Тогда в $U \times \mathbb{D}$ есть гладкая функция $z$, однолистная в $\mathbb{D}$ при каждом фиксированном $\lambda \in U$, равная 0 при $w=0$ и равная $f$ при $1 / 3 \leqslant|w| \leqslant 2 / 3$. Так как $f$ является $J$-голоморфной в $\mathscr{U}$, то структура $J$ в координатах $\lambda_{1}, \ldots, \lambda_{n}, z$ имеет определяющую форму $d z-\mu d \bar{z}-\sum a_{j} d \bar{\lambda}_{j}$ с $\mu=0=a_{j}$ при $1 / 3 \leqslant|z| \leqslant 2 / 3$. Доопределяя $\mu$ и $a_{j}$ нулями при $|z| \geqslant 2 / 3$, получаем комплексную структуру в $U \times(\widehat{\mathbb{C}} \backslash 0)$, для которой сохраним обозначение $J$. Тогда слои проекции $U \times(\widehat{\mathbb{C}} \backslash 0)$ в $U-J$-голоморфные, односвязные и потому конформно эквивалентные $\mathbb{C}$ по теореме Римана.

Здесь удобно заменить $z$ на $\zeta=1 / z$ и перейти к соответствующей структуре $J^{\prime}$ в $U \times \mathbb{C}$. Так как семейство $M$ трансверсально диск-выпуклое, то, уменьшая, если надо $U$, можем считать, что структура $J^{\prime}$ диск-выпуклая над $\partial U$. По теореме 4.1 , существует $J^{\prime}$-голоморфное над $U$ движение, непрерывное над $\bar{U}$, которое заполняет $\partial U \times \mathbb{C}$ и некоторую область $\mathscr{D}$ над $U$ с односвязными слоями. Повторяя шаг 2 доказательства теоремы Нишино 5.4, получаем, что на самом деле $\mathscr{D}=U \times \mathbb{C}$ и $\left(U \times \mathbb{C}, J^{\prime}\right)$ послойно биголоморфно эквивалентно обыкновенному цилиндру $U \times \mathbb{C}$. Возвращаясь к координате $z$, получаем окрестность $V \ni 0$ в $\mathbb{C}$ и послойное непрерывное вложение $F_{0}: U \times V \rightarrow U \times \mathbb{D}$, $J$-голоморфное в $U \times(V \backslash 0)$. Отображение $F_{0}$ задает на $U \times V$ комплексную карту с координатами, $J$-голоморфными в $U \times(V \backslash 0)$.

Так как семейство $M$ локально топологически тривиальное, то, добавляя к каждому слою $M_{\lambda}$ прокол, получаем семейство кругов $\widehat{M}_{\lambda} \supset M_{\lambda}$ над $B$, которое локально имеет топологическую структуру прямого произведения. Это очевидно порождает на множестве $\widehat{M}$ топологию и $\widehat{M}$ становится топологическим многообразием. Положим $S=\widehat{M} \backslash M$ и естественно определим $\hat{\pi}\left(\widehat{M}_{\lambda}\right)=\lambda$.

По доказанному, для каждой точки $p \in S$ существуют окрестность $W \ni p$ в $\widehat{M}$ и послойный гомеоморфизм $W \rightarrow \hat{\pi}(W) \times \mathbb{D}$ такой, что сужение $W \backslash S \rightarrow$ $\hat{\pi}(W) \times(\mathbb{D} \backslash 0)$ голоморфно. Покроем $S$ системой таких окрестностей $W_{\alpha} ;$ координатные отображения, описанные выше, обозначим соответственно через $F_{\alpha}$. Если $W_{\alpha} \cap W_{\beta}$ непусто, то отображение $F_{\beta} \circ F_{\alpha}^{-1}$ является тогда послойным гомеоморфизмом $F_{\alpha}\left(W_{\alpha} \cap W_{\beta}\right) \rightarrow F_{\beta}\left(W_{\alpha} \cap W_{\beta}\right)$, голоморфным вне $F_{\alpha}(S)$ и потому голоморфным во всем $F_{\alpha}\left(W_{\alpha} \cap W_{\beta}\right)$ по теореме об устранимой особенности. Таким образом, система описанных комплексных карт, покрывающих окрестность $S$ в $\widehat{M}$, определяет в этой окрестности структуру комплексного многообразия, которая вместе с таковой на $M$ задает комплексную структуру на многообразии $\widehat{M}$.

Голоморфность $S$ - локальное свойство, доказанное выше. Теорема доказана. 
Вопрос об униформизуемости построенного семейства $\widehat{M}$ никак не связан с теоремой, это отдельная общая проблема униформизации косых цилиндров. Одной псевдовыпуклости и даже штейновости многообразия $\widehat{M}$ недостаточно (см. пример в [28]), нужны дополнительные условия.

СлеДСтвиЕ 6.1. Пусть $\pi: M \rightarrow B$ - трансверсалъно диск-выпуклое голоморфное семейство римановых поверхностей, конформно эквивалентных проколотой плоскости $\mathbb{C} \backslash 0$, над конечномерным комплексным многообразием $B$. Тогда семейство $M$ локально голоморфно тривиальное. Оно глобально тривиальное, если В односвязное и на нем разрешима вторая проблема Кузена.

ДокАЗАТЕЛЬство. Над каждым координатным шаром $U \subset B$ семейство $M$ топологически тривиальное, и, значит, $\pi^{-1}(U)$ биголоморфно послойно эквивалентно многообразию $(U \times(\mathbb{C} \backslash 0), J)$ с некоторой гладкой согласованной комплексной структурой $J$. Повторяя доказательство теоремы, продолжаем $J$ до комплексной структуры $\hat{J}$ в $U \times \widehat{\mathbb{C}}$. Таким образом, в общем случае получается голоморфное семейство сфер $\hat{\pi}: \widehat{M} \rightarrow B$ и голоморфное послойное вложение $F: M \rightarrow \widehat{M}$, причем $\widehat{M} \backslash F(M)$ локально (по $\lambda \in B$ ) состоит из непересекающихся образов двух голоморфных сечений проекции $\hat{\pi}$. Глобально, многообразие $\widehat{M} \backslash F(M)$, двулистно накрывающее $B$, может быть связным, но если $B$ односвязное, то оно состоит из образов двух глобальных голоморфных сечений $\hat{\pi}$.

Остальное следует из теоремы 1.1. Следствие доказано.

6.2. Устранение послойно дискретных особенностей. Следующее утверждение является абстрактным аналогом теоремы Э. Леви об аналитичности особенностей (см. [51]).

Лемма 6.1. Пусть $E$ - замкнутое подмножество бикруга $\mathbb{D} \times \mathbb{D}$, слои $E_{\lambda}$, $\lambda \in \mathbb{D}$, которого имеют не более $k$ точек, причем проекиия $E$ в $\mathbb{D}, E_{\lambda} \rightarrow \lambda$, собственная, а множество $U=\left\{\lambda: \# E_{\lambda}=k\right\}$ непустое и открытое. Пусть $J$ - непрерывная комплексная структура в $\mathbb{D}^{2} \backslash E$, согласованная с проекиией в $\mathbb{D}$, трансверсально диск-выпуклая над $U$ и такая, что римановы поверхности $\left(\mathbb{D} \backslash E_{\lambda}, J\right)$ конформно эквивалентны кругу с не более чем $k$ проколами. Тогда существует непрерьвная комплексная структура $\hat{J}$ в $\mathbb{D}^{2}$, совпадающая c $J$ в $\mathbb{D}^{2} \backslash$ Е и такая, что $E$ - комплексно-аналитическое подмножество комплексного многообразия $\left(\mathbb{D}^{2}, \hat{J}\right)$.

(Комплексная структура в области в $\mathbb{C}^{n}$ называется непрерывной, гладкой и т. п., если функции, голоморфные относительно этой структуры, непрерывные, гладкие и т. п.)

ДокАзАТЕЛЬство. Утверждение достаточно доказать в окрестности каждой точки $E$; функции перехода новых координатных карт будут голоморфными, как в конце доказательства теоремы 6.1. Поэтому, по теореме Сью [55] и рассуждая далее как в теореме 6.1, можно считать, что существует непрерывная вертикальная координата $\zeta$ в $\mathbb{D} \times \mathbb{D}$ такая, что структура $J$ относительно координат $\lambda, \zeta$ совпадает с обычной комплексной структурой в $\mathbb{D} \times\{1 / 2<$ $|\zeta|<1\}$ и таким образом продолжается до обычной комплексной структуры в $\mathbb{D} \times\left(\mathbb{C} \backslash \frac{1}{2} \overline{\mathbb{D}}\right)$ и $E \subset \mathbb{D} \times \frac{1}{2} \mathbb{D}$. 
По условию, для каждой $\lambda \in \mathbb{D}$ существует функция $w(\lambda, \cdot)$, непрерывная в $\overline{\mathbb{D}}$, биголоморфно отображающая $\left(\mathbb{D} \backslash E_{\lambda}, J\right)$ на $\mathbb{D} \backslash E_{\lambda}^{\prime}$, где $\# E_{\lambda}^{\prime}=\# E_{\lambda}$, и однозначно определяемая условием, что точки $\pm 1, i$ при этом отображении неподвижны.

Функция $w(\lambda, \zeta)$ непрерывна в $\mathbb{D}^{2}$. В самом деле, пусть $\lambda_{j} \rightarrow \lambda_{0}$. Так как функции $w\left(\lambda_{j}, \cdot\right)$ равномерно ограничены и $\mathbb{D}^{2} \backslash E$ - комплексное многообразие, то, используя локальные $J$-голоморфные координаты (среди которых $\lambda$ ) и переходя к подпоследовательности, можем считать, что последовательность функций $w\left(\lambda_{j}, \cdot\right)$ сходится равномерно внутри $\mathbb{D} \backslash E_{\lambda_{0}}$ к некоторой $J$-голоморфной функции $w^{0}$. Так как все $w\left(\lambda_{j}, \cdot\right)$ голоморфно и однолистно продолжаются в кольцо $1 / 2<|\zeta|<2$ по принципу симметрии и имеют там неподвижные точки $\pm 1, i$, то функция $w^{0}$ не постоянная и однолистная. Множество предельных значений $w^{0}$ на $E_{\lambda_{0}}$ конечно (по условию), $\left|w^{0}\right|=1$ на $\partial \mathbb{D}$ и поэтому $w^{0}=w\left(\lambda_{0}, \cdot\right)$ (функция $w^{0} \circ w\left(\lambda_{0}, \cdot\right)^{-1}$ голоморфна в $\mathbb{D}$ по теореме об устранимой особенности, поэтому она дробно-линейная и имеет неподвижные точки $\pm 1, i)$.

Фиксируем $\lambda_{0} \in U$. По следствию 6.1 , существуют круг $V \ni \lambda_{0}$ в $U$ и комплексная структура $\hat{J}$ в $V \times \mathbb{C}$, совпадающая с $J$ в $(V \times \mathbb{C}) \backslash E$ (этим условием она определена однозначно) и такая, что $E$ над $V$ состоит из $k \hat{J}$-голоморфных графиков. При $k=1$ утверждение доказано в теореме 6.1 , поэтому далее считаем, что $k>1$. По теореме об устранимой особенности, функция $w(\lambda, \zeta)$ является $\hat{J}$-голоморфной в $\lambda \times 2 \mathbb{D}$ при любом фиксированном $\lambda \in U$.

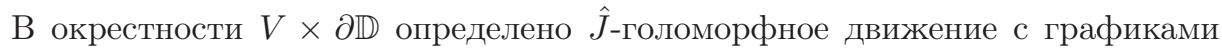
$\zeta=$ const. Уменьшая, можем считать в силу леммы 1.1 , что в $V \times 2 \mathbb{D}$ есть

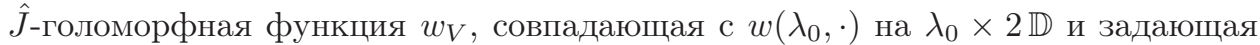
голоморфное послойное вложение $(V \times \overline{\mathbb{D}}, \hat{J})$ в $V \times \mathbb{C}$. Поэтому, еще уменьшая $V$ (если надо), по теореме Берса-Ройдена (теорема 3.3$)$ получаем $\hat{J}$-голоморфное движение $\zeta=\phi(\lambda, z)$, заполняющее $V \times \overline{\mathbb{D}}$, с графиками $\zeta=$ const в окрестности $V \times \partial \mathbb{D}$. Обозначим через $\mu$ его характеристику, соответствующую фиксированному биголоморфизму $f_{0}:=w(\lambda, \cdot): \mathbb{D} \rightarrow\left(\lambda_{0} \times \mathbb{D}, \hat{J}\right)$.

Так как отображение $\lambda \mapsto \mu(\lambda, \cdot)$ голоморфно в $V$ (лемма 2.5), то для каждого $\lambda \in V$, по теореме Альфорса-Берса [1], [2], существуют два канонических гомеоморфных в $\mathbb{D}$ решения уравнения Бельтрами $w_{\bar{z}}=\mu(\lambda, \cdot) w_{z}$ : одно, $w^{\mu}(\lambda, \cdot)$, голоморфное по $\lambda \in V$ (как в лемме 2.6$)$, и другое, $w_{\mu}(\lambda, \cdot): \mathbb{D} \rightarrow \mathbb{D}$, с неподвижными точками $\pm 1, i$, гладкое (и даже $\mathbb{R}$-аналитическое) по $\lambda$. Так как $w^{\mu}$ и $w_{\mu}$ - решения одного уравнения Бельтрами и отображения $\lambda \mapsto w^{\mu}(\lambda, \cdot)$, $\lambda \mapsto w_{\mu}(\lambda, \cdot)$ гладкие, то существует гладкая в соответствующей области функция $h(\lambda, \eta)$, голоморфная по $\eta$ и такая, что $w^{\mu}(\lambda, z) \equiv h\left(\lambda, w_{\mu}(\lambda, z)\right)$ в $V \times \overline{\mathbb{D}}$.

Положим $\psi_{1}(\lambda, \zeta)=\phi(\lambda, \cdot)^{-1}(\zeta)$. По определению характеристики (п. 2.2),

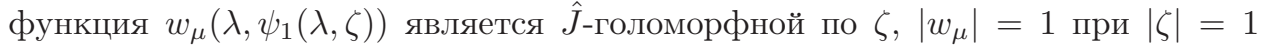
и точки $\pm 1, i$ неподвижные. Так как функция $w(\lambda, \zeta)$, определенная выше, такая же, то $w_{\mu}\left(\lambda, \psi_{1}(\lambda, \zeta)\right) \equiv w(\lambda, \zeta)$. Обозначая $w(\lambda, \cdot)^{-1}(\eta):=\psi_{2}(\lambda, \eta)$, получаем, что $w_{\mu}\left(\lambda, \psi_{1}\left(\lambda, \psi_{2}(\lambda, w)\right)\right) \equiv w$ в $\overline{\mathbb{D}}$ при любом $\lambda \in V$. Следовательно, $w^{\mu}\left(\lambda, \psi_{1}\left(\lambda, \psi_{2}(\lambda, w)\right)\right) \equiv h(\lambda, w)$ в $V \times \overline{\mathbb{D}}$, т. е. функция $w^{\mu}$ гладкая относительно координат $(\lambda, w)$ в $V \times \overline{\mathbb{D}}$. Так как локально $\hat{J}$-голоморфные функции разлагаются в степенные ряды по $\lambda$ и $w^{\mu}$, то отсюда следует, что структура $\hat{J}$ в $U \times \overline{\mathbb{D}}$ гладкая относительно координат $(\lambda, w)$. 
Так как функция $w(\lambda, \zeta)$ является $\hat{J}$-голоморфной на слоях $\lambda \times \overline{\mathbb{D}}$, то определяющая форма структуры $\hat{J}$ в $U \times \mathbb{D}$ относительно координат $\lambda, w$ имеет вид $d w-a(\lambda, w) d \bar{\lambda}$ с гладкой функцией $a=-h_{\bar{\lambda}} / h_{w}$, голоморфной по $w$ и равномерно ограниченной в $U \times r \mathbb{D}$ для любого $r<1$. (Голоморфность $a$ по $w$ эквивалентна интегрируемости $J$ как почти комплексной структуры, см. начало п. 1.3.)

Над каждым кругом $V \subset U$ множество $E \cap(V \times \mathbb{D})$ является объединением $k$

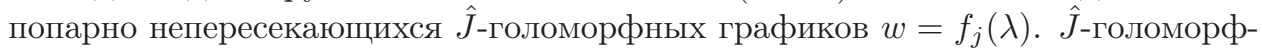
ность означает, что каждая $f_{j}$ является решением над $V$ уравнения $f_{\bar{\lambda}}=a(\cdot, f)$ (см. п. 4.1). Функция $F:=\prod_{i \neq j}\left(f_{i}-f_{j}\right)^{2}$ определена уже на всем множестве $U$ и стремится к нулю при $\lambda \rightarrow \partial U \cap \mathbb{D}$, так как над точками из $\partial U \cap \mathbb{D}$ множества $E_{\lambda}$ содержат меньше чем $k$ точек (и проекция $E$ в $\mathbb{D}$ собственная по условию). Так как

$$
F_{\bar{\lambda}}=2 \sum_{i \neq j} \frac{a\left(\cdot, f_{i}\right)-a\left(\cdot, f_{j}\right)}{f_{i}-f_{j}} F
$$

в $V$, а функция $a$ ограничена и голоморфна по $w\left(\right.$ и $\left.\left|f_{j}\right| \leqslant 1 / 2\right)$, то существует непрерывная и ограниченная в $U$ функция $C$ такая, что $F_{\bar{\lambda}}=C F$. Доопределяя $C$ нулем вне $U$ и полагая $c=T C$, где $T$ - плоский интеграл Коши (оператор, обратный к $\partial / \partial \bar{\lambda}$, см. п. 4.1), мы получаем, что $c_{\bar{\lambda}}=C$ и потому функция $e^{-c} F$ голоморфна в $U$. Доопределяя $F$ нулем в $\mathbb{D} \backslash U$, получаем непрерывную в $\mathbb{D}$ функцию $e^{-c} F$, голоморфную вне своих нулей. По теореме Радо, эта функция голоморфна в $\mathbb{D}$, и, следовательно, множество $\mathbb{D} \backslash U$ дискретное.

Таким образом, остается показать, что $\hat{J}$ продолжается до комплексной структуры в окрестности изолированных точек $\left(\lambda_{0}, w_{0}\right) \in E$ с $\lambda_{0} \in \mathbb{D} \backslash U$, вне которых структура уже есть.

Заменяя $a$ на $\chi a$, где $\chi$ - гладкая функция с носителем в $\mathbb{D}^{2}$, равная 1 в окрестности $\left(\lambda_{0}, w_{0}\right)$, мы получаем почти комплексную структуру $J^{\prime}$ в $\widehat{\mathbb{C}} \times \mathbb{C}$ с определяющей формой $d w-\chi a d \bar{\lambda}$, удовлетворяющей условиям следствия 4.2. Поэтому $\widehat{\mathbb{C}} \times \mathbb{C}$ заполняется $J^{\prime}$-голоморфным движением. В окрестности $\left(\lambda_{0}, w_{0}\right)$,

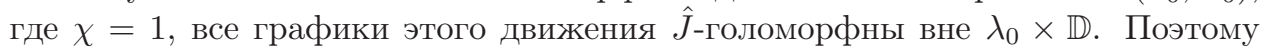
существует круг $V \ni \lambda_{0}$ в $\mathbb{D}$ и окрестность $W \ni\left(\lambda_{0}, w_{0}\right)$ такие, что суже-

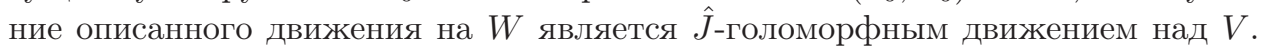
По лемме 2.5, характеристика этого движения (соответствующая тождественному отображению $w: \mathbb{D} \rightarrow \mathbb{D}$ ) голоморфна по $\lambda \in V \backslash \lambda_{0}$ и потому, по теореме об устранимой особенности, голоморфно по $\lambda$ продолжается в $V \times W_{\lambda_{0}}$. По лемме 2.6, существует послойный гомеоморфизм $W$ на некоторую область $\Omega \subset V \times \mathbb{C}, J$-голоморфный в $W \backslash\left(\lambda_{0} \times \mathbb{D}\right)$, т. е. существует непрерывная в $W$

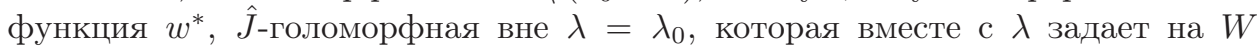
комплексную карту. Опять же по теореме об устранимой особенности, $w^{*}$ яв-

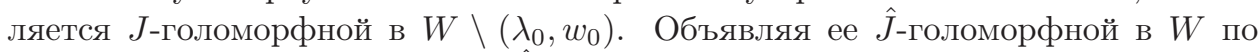
определению, мы продолжаем $\hat{J}$ до комплексной структуры в $W$, порождаемой голоморфными функциями от $\lambda$ и $w^{*}$. Лемма доказана.

Одномерность базы в доказательстве используется по существу лишь для простого решения $\bar{\partial}$-проблемы. Используя в полной мере условие интегрируемости $J$ и линейные операторы решения $\bar{\partial}$-проблемы, скажем, в шаре в $\mathbb{C}^{n}$, 
доказанную лемму можно распространить на случай любой конечномерной комплексной базы $B$ (вместо $\mathbb{D})$. Однако это требует дополнительного исследования квазилинейных $\bar{\partial}$-уравнений в многомерных областях и мы оставляем это на будущее.

Теорема 6.2. Пусть $\pi: M \rightarrow \mathbb{D}$ - голоморфное семейство римановых поверхностей $M_{\lambda}$, конформно эквивалентных кругу с конечным числом проколов (зависящих от $\lambda$ ), причем число проколов равномерно ограничено для всех $\lambda \in \mathbb{D}$. Предположим также, что существует замкнутое подмножество $X \subset M$ такое, что проекиия $\pi \mid \partial X \rightarrow \mathbb{D}$ собственная и область $M \backslash X$ послойно гомеоморфна $\mathbb{D} \times\left(\mathbb{D} \backslash \frac{1}{2} \overline{\mathbb{D}}\right)$. Тогда, если семейство $M$ является трансверсальным и вертикально диск-выпуклым (например, если многообразие $M$ псевдовыпуклое), то существуют комплексное многообразие $\widehat{M}$, голоморфная проекиия $\hat{\pi}: \widehat{M} \rightarrow \mathbb{D}$ со слоями, конформно эквивалентными кругу, и голоморфное послойное вложение $F: M \rightarrow \widehat{M}$ такие, что $\widehat{M} \backslash F(M)$ есть комплексно-аналитическое подмножество в $\widehat{M}$.

ДокАзАтельство. Многообразие $\widehat{M}$ определим как $\left(\mathbb{D}^{2}, \hat{J}\right)$ с непрерывной комплексной структурой $\hat{J}$, которую будем строить, послойно вкладывая $M$ в $\mathbb{D}^{2}$.

По условию, существует послойный гомеоморфизм $f: M \backslash X \rightarrow \mathbb{D} \times\left(\mathbb{D} \backslash \frac{1}{2} \overline{\mathbb{D}}\right)$. Окружность $M_{\lambda} \cap\{|f(\lambda, \cdot)|=2 / 3\}$ на каждом $M_{\lambda}$ ограничивает область $M_{\lambda}^{\prime}$, конформно эквивалентную кругу с конечным числом проколов. Обозначим через $w_{\lambda}$ функцию, конформно отображающую $M_{\lambda}^{\prime}$ на $\frac{2}{3} \mathbb{D} \backslash E_{\lambda}$, \# $E_{\lambda}<\infty$, и однозначно определяемую нормировкой $w\left(M_{\lambda} \cap\{f=c\}\right)=c$ для $c= \pm 2 / 3,(2 / 3) i$. Из единственности такого отображения так же, как в доказательстве леммы 6.1 , следует, что функция $w$, равная $w_{\lambda}$ на $M_{\lambda}^{\prime}$, непрерывна на области $M^{\prime}=$ $\bigsqcup M_{\lambda}^{\prime}$. При помощи гомеоморфизма $f$ она естественно продолжается до непрерывной функции на $M$, однолистной на слоях и такой, что $p \mapsto(\lambda(p), w(p))-$ гомеоморфизм $M$ на $\mathbb{D}^{2} \backslash E$, где $E$ - замкнутое подмножество с конечными слоями $E_{\lambda}$. Комплексная структура на $M$ индуцирует непрерывную комплексную структуру $J$ на $\mathbb{D}^{2} \backslash E$. Покажем, что она удовлетворяет условию леммы 6.1 . Так как семейство $M$ трансверсально диск-выпуклое, то достаточно показать, что множество $U=\left\{\lambda: \# E_{\lambda}=k\right\}$, где $k=\sup \# E_{\lambda}$, открыто в $\mathbb{D}$.

Предположим противное. Тогда существуют $\lambda_{0} \in \mathbb{D}$ и последовательность точек $\lambda_{j} \rightarrow \lambda_{0}$ таких, что \# $E_{\lambda_{j}} \leqslant k-1$. Пусть $V^{i}$ - замкнутые попарно непересекающиеся круги в $\mathbb{D}$, покрывающие окрестность $E_{\lambda_{0}}$, и $f_{0}: \mathbb{D} \backslash E_{\lambda_{0}} \rightarrow M_{\lambda_{0}}-$ конформное отображение. По лемме 1.1 , в окрестности $f_{0}\left(\partial V_{i}\right)$ на $M$ существует голоморфная, однолистная в слоях функция $h$, равная $f_{0}^{-1}$ на $M_{\lambda_{0}}$, с уровнями, трансверсальными слоям $M_{\lambda}$. Поэтому множество $\gamma_{j}^{i}:=\left\{p \in M_{\lambda_{j}}: h(p) \in\right.$ $\left.\partial V^{i}\right\}$ для каждого $i$ и $j \gg 1$ является замкнутой жордановой кривой. Так как $M_{\lambda}$ имеют род 0 (конформно эквивалентны плоским областям) и число этих окружностей на $M_{\lambda_{j}}$ больше числа проколов, то, переходя к подпоследовательности и перенумеровав $V^{i}$, можно считать, что каждая кривая $\gamma_{j}^{1}$ ограничивает в $M_{\lambda_{j}}$ голоморфный диск $D_{j}$. Границы этих дисков равномерно сходятся при $j \rightarrow \infty$ к $\gamma_{0}^{1}$. По условию вертикальной диск-выпуклости, подходяще параметризованные диски $D_{j}$ должны равномерно сходиться к некоторому диску в $M_{\lambda_{0}}$ с границей $\gamma_{0}^{1}$. Но $\gamma_{0}^{1}$ ограничивает на $M_{\lambda_{0}}$ лишь проколотый диск $f_{0}\left(V^{1} \backslash E_{\lambda_{0}}\right)$, 
и это противоречие показывает, что множество $U$ открытое. Остальное следует из леммы 6.1. Теорема доказана.

ЗАмЕчАниЕ 6.1. Условие равномерной ограниченности числа проколов в теореме излишне. Его несложно вывести из вертикальной диск-выпуклости и доказательства леммы 6.1, но я не вижу в этом особого интереса.

6.3. Комментарии. 1. Представляется правдоподобным, что для семейств римановых поверхностей без края условие диск-плоской границы в теореме 5.3 можно заменить более слабым “односторонним” условием трансверсальной дисквыпуклости (и тогда, по теореме 3.2 , ее можно распространить на семейства над любой базой $B)$.

2. Интересно, можно ли в теореме 5.1 убрать условие существования комплексной структуры на самом множестве $E$, т. е. обобщить теорему 6.1 на топологически тривиальные семейства $M$, слои $M_{\lambda}$ которых конформно эквивалентны $\mathbb{D} \backslash E_{\lambda}$, где $E_{\lambda}$ - замкнутые дискретные подмножества $\mathbb{D}$. В этой связи приведем следующую теорему Ямагучи [61].

Пусть $\pi: M \rightarrow \mathbb{D}$ - топологически тривиальное голоморфное семейство римановых поверхностей $M_{\lambda}$, конформно эквивалентных соответственно $\widehat{\mathbb{C}} \backslash$ $E_{\lambda}$, где $E_{\lambda}$ - замкнутые подмножества сферь Римана, устранимые для голоморфных функций с конечным интегралом Дирихле. Тогда, если $M-$ многообразие Штейна, то оно биголоморфно послойно эквивалентно некоторой области $\Omega \subset \mathbb{D} \times \widehat{\mathbb{C}}$.

(Условие на $E_{\lambda}$ здесь означает, что не существует непостоянных голоморфных в $\widehat{\mathbb{C}} \backslash E_{\lambda}$ функций $f$ таких, что $\int i d f \wedge d \bar{f}<+\infty$; такие множества вполне разрывны, но их хаусдорфова размерность может равняться 2.)

Это теорема - одновременно и о продолжении комплексной структуры, и об униформизации. По лемме 5.1 , множество $(\mathbb{D} \times \widehat{\mathbb{C}}) \backslash \Omega$ заполняется голоморфным (мероморфным) движением, нормируя которое, область $\Omega$ переводим в $\mathbb{D} \times \mathbb{C}$ и тогда дополнение к $\Omega$ состоит из попарно не пересекающихся голоморфных графиков над $\mathbb{D}$.

Условие на $E_{\lambda}$ в теореме Ямагучи локальное, поэтому она частично обобщается до следующей теоремы о продолжении комплексной структуры.

Пусть $\pi: M \rightarrow \mathbb{D}$ - топологически тривиальное голоморфное семейство римановых поверхностей $M_{\lambda}$, конформно эквивалентных $\mathbb{D} \backslash E_{\lambda}$, где $E_{\lambda}$ устранимы для голоморфных функиий в $\mathbb{D} \backslash E_{\lambda}$ с конечным интегралом Дирихле. Предположим, что $M$ есть многообразие Штейна. Тогда существуют комплексное многообразие $\widehat{M}$ с сюргективной голоморбной проекиией $\hat{\pi}: \widehat{M} \rightarrow \mathbb{D}$ и голоморфное послойное вложение $\iota: M \rightarrow \widehat{M}$ такое, что $\widehat{M} \backslash \iota(M)$ есть объединение попарно непересекающихся образов голоморфных сечений проекиии $\hat{\pi}$.

НАБРОСОК ДОКАЗАТЕЛЬСТВА. Так как семейство топологически тривиальное, то $M$ послойно гомеоморфно множеству $\mathbb{D} \times\left(\mathbb{D} \backslash E_{0}\right)$, где $E_{0}-$ замкнутое вполне разрывное подмножество в $\mathbb{D}$. Таким образом, можно считать, что $M=\left(\mathbb{D} \times\left(\mathbb{D} \backslash E_{0}\right), J\right)$ с соответствующей комплексной структурой $J$. В качестве $\widehat{M}$ берем, естественно, $\mathbb{D}^{2}$. Для произвольной $\lambda_{0} \in \mathbb{D}$, по лемме 1.1 , существует $J$-голоморфная функция $h$ в окрестности $\lambda_{0} \times\left(\mathbb{D} \backslash E_{0}\right)$ в $M$, однолистная 
на слоях и равная 1 по модулю на $\lambda_{0} \times r \partial \mathbb{D}$, где $r<1$ таково, что $E_{0} \subset r \mathbb{D}$. Тогда над малым кругом $V \ni \lambda_{0}$ есть область $W \supset \lambda_{0} \times r \mathbb{D}$ и непрерывная функция $\zeta$, равная $h$ в окрестности множества $\{|h|=1\}$, однолистная в каждом слое и такая, что образ $W$ в координатах $\lambda, \zeta$ равен $V \times \mathbb{D}$. Образ $J$ ввиду $J$-голоморфности $h$ при этом преобразовании совпадает со стандартной комплексной структурой в окрестности $V \times \partial \mathbb{D}$ и, таким образом, продолжается до комплексной структуры в $V \times\left(\widehat{\mathbb{C}} \backslash E_{0}\right)$. По теореме Ямагучи, эта структура продолжается на $V \times \widehat{\mathbb{C}}$, и, следовательно, структура $J$ продолжается до непрерывной комплексной структуры на $W$. Так как $E_{\lambda}$ устранимы для однолистных функций, то такие локальные комплексные карты согласованы на пересечениях.

3. Следующая теорема Майтани-Ямагучи [44] тоже является теоремой об одновременном устранении особенностей комплексных структур и униформизации.

Пусть $\pi: M \rightarrow \mathbb{D}$ - голоморфное семейство римановых поверхностей и $M-$ многообразие Штейна. Обозначим через $X$ множество точек $\lambda \in \mathbb{D}$ таких, что каждая $M_{\lambda}, \lambda \in X$, конформно эквивалентна $\widehat{\mathbb{C}} \backslash E_{\lambda}$, где $E_{\lambda}-$ замкнутое полярное подмножество в $\widehat{\mathbb{C}}$, и предположим, что множество $X$ неполярное. Тогда $X=\mathbb{D}$ и $M$ биголоморфно послойно эквивалентно области в $\mathbb{D} \times \widehat{\mathbb{C}}$.

Эта теорема по описанной выше схеме частично распространяется на общие семейства римановых поверхностей.

Пусть $\pi: M \rightarrow \mathbb{D}$ - голоморфное семейство римановых поверхностей и $M-$ многообразие Штейна. Предположим, что каждая $M_{\lambda}$ конформно эквивалентна $\widehat{M}_{\lambda} \backslash E_{\lambda}$, где $\widehat{M}_{\lambda}$ - некоторая риманова поверхность и $E_{\lambda}$ - ее компактное дискретное подмножество. Обозначим через $X$ множество точек $\lambda \in \mathbb{D}$ таких, что $E_{\lambda}$ полярно в $\widehat{M}_{\lambda}$, и предположим, что само $X$ не полярное. Тогда $X=\mathbb{D}$ и на дизгюнктном объединении $\widehat{M}$ множеств $M_{\lambda} \sqcup E_{\lambda}$ существует комплексная структура, совпадающая с таковой на $M=\bigsqcup M_{\lambda}$.

НАБросок Доказательства. Фиксируем $\lambda_{0} \in \bar{X} \cap \mathbb{D}$. Множество $E_{\lambda_{0}}$ покрывается конечным числом кругов $V_{j}$, границы которых не пересекают $E$. Фиксируем $j$. По лемме 1.1 , существует окрестность $W \supset \partial V_{j}$ в $M$, биголоморфно послойно эквивалентная области $U \times A$, где $U-$ окрестность $\lambda_{0}$ и $A-$ кольцо $r<|\zeta|<1 / r$ (см. выше) с обычной комплексной структурой. К этому кольцевому цилиндру послойно подклеивается $U \times(\widehat{\mathbb{C}} \backslash \overline{\mathbb{D}})$, и в результате образуется семейство римановых поверхностей из теоремы Майтани-Ямагучи. По этой теореме, $U \subset X$, и, следовательно, $X=\mathbb{D}$. По той же теореме, на дизъюнктном объединении поверхностей $M_{\lambda} \sqcup E_{\lambda}, \lambda \in U$, вводятся и топология, и комплексная структура. Так как полярные множества устранимы для ограниченных голоморфных функций, то таким образом построенные карты вместе с $M$ задают на $\bigsqcup\left(M_{\lambda} \sqcup E_{\lambda}\right)$ структуру комплексного многообразия, совпадающую с таковой на $M$.

4. При доказательстве леммы 6.1 мы столкнулись с задачей продолжения комплексной структуры в изолированную особенность. Общая постановка здесь такова: 
Пусть $J$ - гладкая комплексная структура в проколотой окрестности $U \backslash 0$ в $\mathbb{C}^{n}$. Найти условия, достаточные для продолжения $J$ до непрерывной комплексной структуры в $U$, т. е. для существования непрерывных в некоторой окрестности $V \ni 0$ функций $f_{1}, \ldots, f_{n}, J$-голоморфных в $V \backslash 0$ и таких, что $V \ni z \mapsto\left(f_{1}, \ldots, f_{n}\right)(z) \in \mathbb{C}^{n}-$ вложение.

Простые примеры показывают, что в общем случае это невозможно. По теореме об устранимой особенности, это невозможно, если существует $J$-голоморфная в $V$ функция, разрывная в 0 . Например, если $J$ - структура, порождаемая алгеброй непрерывных в $\mathbb{C}^{n} \backslash 0$ функций с образующими $z_{j}(1+|z|) /|z|$, $j=1, \ldots, n$, то многообразие $\left(\mathbb{C}^{n} \backslash 0, J\right)$ биголоморфно эквивалентно области $\mathbb{C}^{n} \backslash \overline{\mathbb{B}}_{n}$ (дополнению к шару) с обычной структурой, никакого непрерывного продолжения в 0 нет.

Естественно возникает вопрос, не является ли такая разрывность единственным препятствием, и уж очень оптимистическая гипотеза:

Если $J$ - гладкая комплексная структура в $U \backslash 0$ такая, что соответствующая ей почти комплексная структура продолжается до непрерывной почти комплексной структуры в $U$, то $J$ продолжается до комплексной структуры B $U$.

Конечно, это вопрос уже при $n=2$.

Кстати, если условие гипотезы выполнено, то после подходящего $\mathbb{R}$-линейного преобразования $\mathbb{C}^{n}$ почти комплексная структура в окрестности 0 определяется вектором 1-форм $d z+\mu d \bar{z}$ (типа $\left.(1,0)_{J}\right)$, где $\mu$ - непрерывная матрица размера $n \times n, \mu(0)=0$, гладкая в проколотой окрестности 0 .

Вместо этого условия можно предложить еще ряд интересных гипотетически достаточных условий (например, условие полной плюриполярности точки 0 , т. е. существование $J$-плюрисубгармонической функции $u$ в $U \backslash 0$ такой, что $u>-\infty$, но $\left.\lim _{z \rightarrow 0} u(z)=-\infty\right)$. Одна из таких гипотез доказана: если почти комплексная структура в $\mathbb{C}^{2} \backslash 0$, соответствующая $J$, ортогональна относительно евклидовой метрики и в некотором смысле ограничена, то $J$ продолжается до ортогональной комплексной структуры в $\mathbb{C}^{2}$ (см. [50]).

\section{Список литературы}

[1] Л. Альфорс, Лекиии по квазиконформным отображениям, Мир, М., 1969, 133 с.; пер. с англ.: L. V. Ahlfors, Lectures on quasiconformal mappings, Van Nostrand Mathematical Studies, 10, D. Van Nostrand Co., Inc., Toronto-New York-London, 1966, v+146 pp.

[2] L. Ahlfors, L. Bers, "Riemann's mapping theorem for variable metrics", Ann. of Math. (2), 72 (1960), 385-404.

[3] L. Ahlfors, G. Weill, "A uniqueness theorem for Beltrami equation", Proc. Amer. Math. Soc., 13 (1962), 975-978.

[4] K. Astala, T. Ivaniec, G. Martin, Elliptic partial differential equations and quasiconformal mappings in the plane, Princeton Math. Ser., 48, Princeton Univ. Press, Princeton, NJ, 2009, xviii+677 pp.

[5] K. Astala, G. J. Martin, "Holomorphic motions", Papers on analysis, Rep. Univ. Jyväskylä Dep. Math. Stat., 83, Univ. Jyväskylä, Jyväskylä, 2001, 27-40.

[6] M. Audin, J. Lafontaine (eds.), Holomorphic curves in symplectic geometry, Progr. Math., 117, Birkhäuser, Basel, 1994, xii+328 pp. 
[7] Л. Берс, “Одновременная униформизация”, Пространства римановых поверхностей и квазиконформные отображения, ИЛ, М., 1961, 91-96; пер. с англ.: L. Bers, "Simultaneous uniformization", Bull. Amer. Math. Soc., 66 (1960), 94-97.

[8] Л. Берс, "Голоморфные дифференциалы как функции модулей", Пространства римановых поверхностей и квазиконформные отображения, ИЛ, М., 1961, 99-103; пер. с англ.: L. Bers, "Holomorphic differentials as functions of moduli", Bull. Amer. Math. Soc., 67 (1961), 206-210.

[9] Л. Берс, "Униформизация, модули и клейновы группы", УМH, 28:4(172) (1973), 153-198; пер. с англ.: L. Bers, "Uniformization, moduli and Kleinian groups", Bull. London Math. Soc., 4 (1972), 257-300.

[10] L. Bers, "Fiber spaces over Teichmüller spaces", Acta Math., 130:1 (1973), 89-126.

[11] L. Bers, "Holomorphic families of isomorphisms of Möbius groups", J. Math. Kyoto Univ., 26:1 (1986), 73-76.

[12] L. Bers, H. Royden, "Holomorphic families of injections", Acta Math., 157:3-4 (1986), 259-286.

[13] Б. В. Боярский, "Гомеоморфные решения систем Бельтрами", Докл. АН CCCP, 102 (1955), 661-664.

[14] Б. В. Боярский, “Обобщенные решения системы дифференциальных уравнений первого порядка эллиптического типа с разрывными коэффициентами", Матем. сб., 43(85):4 (1957), 451-503.

[15] Е. М. Чирка, "О распространении голоморфных движений", Докл. РАН, 397:1 (2004), 37-40; англ. пер.: Е. M. Chirka, "On the propagation of holomorphic motions", Dokl. Math., 70:1 (2004), 516-519.

[16] Е. М. Чирка, "Вариации теоремы Гартогса", Комплексный анализ и приложения, Сборник статей, Тр. МИАН, 253, Наука, М., 2006, 232-240; англ. пер.: E. M. Chirka, "Variations of Hartogs' theorem", Proc. Steklov Inst. Math., 253 (2006), 212-220.

[17] Е. М. Чирка, "Пространства Тейхмюллера", Лекц. курсы НОЦ, 15, МИАН, М., 2010, 3-150.

[18] Е. М. Чирка, "Неравенства Харнака, расстояния Кобаяши и голоморфные движения", Тр. МИАН, 279 (2012), 206-216.

[19] C. J. Earle, "On holomorphic families of pointed Riemann surfaces", Bull. Amer. Math. Soc., 79 (1973), 163-166.

[20] C. J. Earle, R. S. Fowler, "Holomorphic families of open Riemann surfaces", Math. Ann., 270:2 (1985), 249-273.

[21] C. J. Earle, R. S. Fowler, "A new characterization of infinite dimensional Teichmüller spaces", Ann. Acad. Sci. Fenn. Ser. A I Math., 10 (1985), 149-153.

[22] C. Ehresmann, "Les connexions infinitésimales dans un espace fibré différentiable", Colloque de topologie (espaces fibrés) (Bruxelles, 1950), Georges Thone, Paris; Masson et Cie., 1951, 29-55.

[23] W. Fischer, H. Grauert, "Lokal-triviale Familien kompakter komplexer Mannigfaltigkeiten", Nachr. Akad. Wiss. Göttingen Math.-Phys. Kl. II, 1965 (1965), 89-94.

[24] F. Forstnerič, "Holomorphic hulls of sets fibered over the circle", Indiana Univ. Math. J., 37 (1988), 869-889.

[25] R. C. Ganning, R. Narasimhan, "Immersion of open Riemann surfaces", Math. Ann., 174 (1967), 103-108.

[26] F. Gardiner, Teichmüller theory and quadratic differentials, Pure Appl. Math. (N. Y.), John Wiley \& Sons, Inc., New York, 1987, xviii+236 pp.

[27] F. Gardiner, Y. Jiang, Z. Wang, "Holomorphic motions and related topics", Geometry of Riemann surfaces, London Math. Soc. Lecture Note Ser., 368, Cambridge Univ. Press, Cambridge, 2010, 156-193. 
[28] A. Glutsyuk, "Nonuniformizable skew cylinder. A counterexample to the simultaneous uniformization problem", C. R. Acad. Sci. Paris Sér. I Math., 332:3 (2001), 209-214.

[29] A. Glutsyuk, "On simultaneous uniformization and local nonuniformizability", C. R. Acad. Sci. Paris, 334 (2002), 489-494.

[30] P. Griffiths, "Complex-analytic priperties of certain Zariski open sets on algebraic varieties", Ann. of Math. (2), 94:1 (1971), 21-51.

[31] M. Gromov, "Pseudo-holomorphic curves in symplectic manifolds", Invent. Math., 82 (1985), 307-347.

[32] A. Grothendieck, "Techniques de construction en géomètrie analytique", Familles d'espaces complexes et fondements de la géométrie analytique, Séminaire H. Cartan, 13, 1960/61, № 7-17, Paris, 1962.

[33] У. Хейман, П. Кеннеди, Субгармонические функиии, т. 1, Мир, М., 1980, 304 с.; пер. с англ.: W. K. Hayman, P. B. Kennedy, Subharmonic functions, v. 1, London Math. Soc. Monographs, 9, Academic Press [Harcourt Brace Jovanovich, Publishers], London-New York, 1976, xvii+284 pp.

[34] J. Hubbard, "Sur la non-existence de sections analytiques à la courbe universelle de Teichmüller", C. R. Acad. Sci. Paris, Ser. A-B, 274 (1972), A978-A979.

[35] Ю. С. Ильяшенко, "Слоения на аналитические кривые", Матем. сб., 88:4 (1972), 558-577; англ. пер.: Yu.S. Ilyashenko, "Fiberings into analytic curves", Math. USSR-Sb., 17:4 (1972), 551-569.

[36] Yu. S. Ilyashenko, "Covering manifolds for analytic families of leaves of foliations by analytic curves", Topol. Methods Nonlinear Anal., 11:2 (1998), 361-373.

[37] Ю. С. Ильяшенко, А. А. Щербаков, "О косых цилиндрах и одновременной униформизации", Дифференциальные уравнения с вещественным и комплексным временем, Сборник статей, Тр. МИАН, 213, Наука, М., 1997, 112-122; англ. пер.: Yu.S. Il'yashenko, A.A. Shcherbakov, "Differential equations with real and complex time", Proc. Steklov Inst. Math., 213 (1966), 104-114.

[38] Ш. Кобаяши, "Гиперболические многообразия и голоморфные отображения", Математика (сб. пер.), 17, № 1, ИЛ, М., 1973, 47-96; англ. пер.: S. Kobayashi, Hyperbolic manifolds and holomorphic mappings, Pure Appl. Math., 2, Marcel Dekker, Inc., New York, 1970, ix+148 pp.

[39] W. Kraus, "Über den Zusammenhang einiger Charakteristiken eines einfach zusammenhängen Bereichen mit der Kreisabbildungen", Mitt. Math. Semin. Univ. Giessen, 21 (1932), 1-28.

[40] M. Kuranishi, Deformations of compact complex manifolds, Séminaire de Mathématiques Supérieures, 39, Les Presses de l'Université de Montréal, Montréal, Que., 1971, 99 pp.

[41] O. Lehto, Univalent functions and Teichmüller spaces, Grad. Texts in Math., 109, Springer-Verlag, New York, 1987, xii+257 pp.

[42] O. Lehto, K. I. Virtanen, Quasikonforme Abbildungen, Springer-Verlag, Berlin-New York, 1965, xi+269 pp.

[43] S. Leng, Introduction to complex hyperbolic spaces, Spriger-Verlag, New York, 1987, viii+271 pp.

[44] F. Maitani, H. Yamaguchi, "Variation of Bergman metrics on Riemann surfaces", Math. Ann., 330:3 (2004), 477-489.

[45] R. Mañé, P. Sad, D. Sullivan, "On the dynamics of rational maps", Ann. Sci. École Norm. Sup. (4), 16:2 (1983), 193-217.

[46] G. Meigniez, "Submersions et fibrations localement triviales", C. R. Acad. Sci. Paris Sér. I Math., 321:10 (1995), 1363-1365.

[47] S. Nag, The complex analytic theory of Teichmüller spaces, Canad. Math. Soc. Ser. Monogr. Adv. Texts, J. Wiley \& Sons, Inc., New York, 1988, xiv+427 pp. 
[48] Z. Nehari, "The Schwarzian derivative and schlicht functions", Bull. Amer. Math. Soc., 55 (1949), 545-551.

[49] T. Nishino, "Nouvelles recherches sur les fonctions entières de plusieurs variables complexes. II. Fonctions entières qui se réduisent à celles d'une variable", J. Math. Kyoto Univ., 9 (1969), 221-274.

[50] S. Salamon, J. Viaclovsky, "Orthogonal complex structures on domains in $\mathbb{R}^{4}$ ", Math. Ann., 343:4 (2009), 853-899; 2007, arXiv: 0704.3422.

[51] Б. В. Шабат, Введение в комплексный анализ, т. 1, 2, 3-е изд., Наука, М., 1985, 336, 464 с.; англ. пер.: В. V. Shabat, Introduction to complex analysis. Part II. Functions of several variables, Transl. Math. Monogr., 110, Amer. Math. Soc., Providence, RI, 1992, x+371 pp.

[52] А. А. Щербаков, “Метод исчерпания косых цилиндров”, Алгебра и анализ, 12:5 (2000), 178-206; англ. пер.: А. A. Shcherbakov, "The exhaustion method for skew cylinders", St. Petersburg Math. J., 12:5 (2001), 847-867.

[53] N. Shcherbina, "Pluripolar graphs are holomorphic", Acta Math., 194:2 (2005), 203-216.

[54] А.И. Шнирельман, "Степень квазилинейчатого отображения и нелинейная задача Гильберта", Матем. сб., 89(131):3(11) (1972), 366-389; англ. пер.: A. I. Šnirel'man, "The degree of a quasi-ruled mapping and the nonlinear Hilbert problem", Math. USSR-Sb., 18:3 (1972), 373-396.

[55] Y.-T. Siu, "Every Stein subvariety admits a Stein neighbourhood", Invent. Math., 38:1 (1976/77), 89-100.

[56] Z. Slodkowski, "Holomorphic motions and polynomial hulls", Proc. Amer. Math. Soc., 111:2 (1991), 347-355.

[57] Z. Slodkowski, "Extensions of holomorphic motions", Ann. Scuola Norm. Sup. Pisa Cl. Sci. (4), 22:2 (1995), 185-210.

[58] T. Sugawa, "The Bers projection and $\lambda$-lemma", J. Math. Kyoto Univ., 32:4 (1992), 701-713.

[59] D. Sullivan, W. Thurston, "Extending holomorphic motions", Acta Math., 157:1 (1986), 243-257.

[60] И.Н. Векуа, Обобщенные аналитические функции, 2-е изд., Наука, М., 1988, 510 с.; англ. пер. 1-го изд.: I. N. Vekua, Generalized analytic functions, Pergamon Press, London-Paris-Frankfurt; Addison-Wesley Publishing Co., Inc., Reading, MA, 1962, xxix+668 pp.

[61] H. Yamaguchi, "Variations de surfaces de Riemann", C. R. Acad. Sci. Paris Sér. A, 286:23 (1978), A1121-A1124.

[62] H. Yamaguchi, "Variations of pseudoconvex domains over $\mathbb{C}^{n "}$, Michigan Math. J., 36:3 (1989), 415-457.

Е. М. Чирка (Е. М. Chirka)

Математический институт им. В. А. Стеклова РАН

E-mail: chirka@mi.ras.ru
Поступила в редакцию 08.10 .2012 